Keywords: WRPS, WTP,

Hanford, Tank Farm Plutonium

Retention: Permanent

\title{
Solids Accumulation Scouting Studies
}

M.R. Duignan

T.J. Steeper

J.L. Steimke

September 2012

Savannah River National Laboratory

Savannah River Nuclear Solutions, LLC

Aiken, SC 29808

Prepared for the U.S. Department of Energy under contract number DE-AC09-08SR22470.

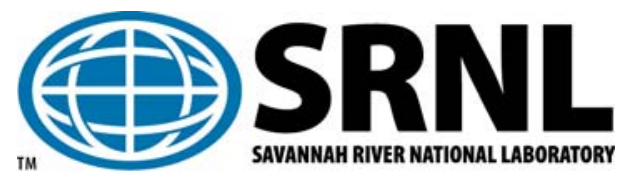




\section{DISCLAIMER}

This work was prepared under an agreement with and funded by the U.S. Government. Neither the U.S. Government or its employees, nor any of its contractors, subcontractors or their employees, makes any express or implied:

1. warranty or assumes any legal liability for the accuracy, completeness, or for the use or results of such use of any information, product, or process disclosed; or

2. representation that such use or results of such use would not infringe privately owned rights; or

3. endorsement or recommendation of any specifically identified commercial product, process, or service.

Any views and opinions of authors expressed in this work do not necessarily state or reflect those of the United States Government, or its contractors, or subcontractors.

\section{Printed in the United States of America \\ Prepared for U.S. Department of Energy}




\section{REVIEWS AND APPROVALS}

\section{AUTHORS:}

M.R. Duignan, Engineering Development Laboratory

Date

T.J. Steeper, Engineering Development Laboratory

Date

J.L. Steimke Engineering Development Laboratory

Date

TECHNICAL REVIEW:

D.J. Adamson, Process Technology Programs

Date

APPROVAL:

B.J. Giddings, Manager

Date

Engineering Development Laboratory

S.L. Marra, Manager

Date

Environmental \& Chemical Process Technology Research Programs

M.G. Thien, Manager

Date

Waste Feed Technical Programs, Washington River Protection Solutions 


\section{ACKNOWLEDGMENTS}

As always, experiments cannot be performed in the Engineering Development Laboratory (EDL) without the assistance of many experienced engineers, technicians, and specialists. Thanks to Mark Fowley who was on the task team in the early stages of work and who assisted in developing the initial plans for the laser positioner and 3-D laser equipment. Mark did a great job to coordinate the demonstration of the 3-D laser equipment from an outside vendor. The laser positioner system that Dale Marzolf and William Cheng put together met the task's needs and became a crucial part of the measurement system; thank you. A special thanks goes to Ken Gibbs, and especially Monica Phillips, for assisting the task with the volume determination technique using photographic images, and for the loan of their imaging equipment. We appreciated the effort by Rich O'Donnell for his initial attempt to create 3-D maps of the accumulated solids mounds from the laser data. With respect to the simulant handling and safety documentation, and its approvals the authors are indebted to Michael Williams and Bridget Miller. Furthermore, we thank Mike handling most of the procurements, especially the simulant solids, in a timely manner. One of the crucial aspects of any experiment is the equipment used to accurately measure the phenomena for which the authors wish to thank William Ryans.

To put together the test facility, run it, take samples, and properly handle those samples takes a series of special talents. A very special thanks goes to Michael Armstrong, the Doc. He was the heart of the operation to execute the equipment and construction changes needed, equipment checkout, facility operation, and the overall day-to-day operations. Without his keen knowledge of where to bang on the pipes to free up the ever present simulant pluggage this test could not have been completed. Vernon Bush was helpful in revising the data acquisition system and to assist Mike when necessary. The patience and care shown by Henry Bolton to measure all the solids samples taken throughout the test were fundamental in eliciting accurate and complete results. This was especially impressive in that Henry last days on this task were some of his last days with SRNS because of his decision to take his well-earned retirement. Sherry Vissage assisted Mike Williams at the start of the task to evaluate many of the new solids when the simulant was being develop and we thank her. Of course, coordinating all this laboratory assistance could not be done efficiently without the assistance of the first line managers, especially Doug Sumpter. When push came to shove Doug was there to push and shove to make sure the task stayed on track.

There are many others that go unnamed, e.g., the Schedulers, Buyers, Subject Matter Experts, Procurement Specialists, Hazardous Material Personnel, Budgeters, Machinists, Safety Evaluators, etc., that supported the entire process to produce a quality product in a safe manner. The authors are also grateful for all this assistance as well as for those who effectively managed the work, i.e., Bill Giddings and Sharon Marra.

Finally, none of this work would have been possible without the confidence of the customer, Washington River Protection Solution (WRPS), especially, Ted Wooley, Pat Lee, and Mike Thien, the manager of Waste Feed Technical Programs. Based on the sound work previously done by Duane Adamson, who is the current technical review, for which we are grateful, Mike Thien chose SRNL to assist him in developing an experimental program in order to answer questions raised by the Defense Nuclear Facilities Safety Board. Thank you. 


\section{EXECUTIVE SUMMARY}

The objective of Solids Accumulation activities was to perform scaled testing to understand the behavior of remaining solids in a Double Shell Tank (DST), specifically AW-105, at Hanford during multiple fill, mix, and transfer operations. It is important to know if fissionable materials can concentrate when waste is transferred from staging tanks prior to feeding waste treatment plants. Specifically, there is a concern that large, dense particles containing plutonium could accumulate in poorly mixed regions of a blend tank heel for tanks that employ mixing jet pumps. At the request of the DOE Hanford Tank Operations Contractor, Washington River Protection Solutions, the Engineering Development Laboratory of the Savannah River National Laboratory performed a scouting study in a 1/22-scale model of a waste staging tank to investigate this concern and to develop measurement techniques that could be applied in a more extensive study at a larger scale. Simulated waste tank solids: Gibbsite, Zirconia, Sand, and Stainless Steel, with stainless steel particles representing the heavier particles, e.g., plutonium, and supernatant were charged to the test tank and rotating liquid jets were used to mix most of the solids while the simulant was pumped out. Subsequently, the volume and shape of the mounds of residual solids and the spatial concentration profiles for the surrogate for heavier particles were measured.

Several techniques were developed and equipment designed to accomplish the measurements needed and they included:

1. Magnetic particle separator to remove simulant stainless steel solids. A device was designed and built to capture these solids, which represent the heavier solids during a waste transfer from a staging tank. [Figure 3-3]

2. Photographic equipment to determine the volume of the solids mounds. The mounds were photographed as they were exposed at different tank waste levels to develop a composite of topographical areas. [Figure 3-12]

3. Laser rangefinders to determine the volume of the solids mounds. The mounds were scanned after tank supernatant was removed. [Figure 3-8]

4. Core sampler to determine the stainless steel solids distribution within the solids mounds. This sampler was designed and built to remove small sections of the mounds to evaluate concentrations of the stainless steel solids at different special locations. [Figure 3-15]

5. Computer driven positioner that placed the laser rangefinders and the core sampler in appropriate locations over solids mounds that accumulated on the bottom of a scaled staging tank where mixing is poor. [Figure 3-6Figure 3-7]

These devices and techniques were effective to estimate the movement, location, and concentrations of the solids representing heavier particles and could perform well at a larger scale

The experiment contained two campaigns with each comprised of ten cycles to fill and empty the scaled staging tank. The tank was filled without mixing, but emptied, while mixing, in seven batches; the first six were of equal volumes of 13.1 gallons each to represent the planned fullscale batches of 145,000 gallons, and the last, partial, batch of 6.9 gallons represented a full-scale partial batch of 76,000 gallons that will leave a 72-inch heel in the staging tank for the next cycle [Table 2-1].

The sole difference between the two campaigns was the energy to mix the scaled staging tank, i.e., the nozzle velocity and jet rotational speed of the two jet pumps. Campaign 1 used $22.9 \mathrm{ft} / \mathrm{s}$, at 
$1.54 \mathrm{rpm}$ [Table 4-1] based on past testing and Campaign 2 used $23.9 \mathrm{ft} / \mathrm{s}$ at $1.75 \mathrm{rpm}$ [Table 4-2], based on visual observation of minimum velocity that allowed fast settling solids, i.e., sand and stainless steel, to accumulate on the scaled tank bottom. Note that the rotation of the jet pumps was in the close-wise direction, looking at the tank from above, and synchronized to rotate together. Over the 26 days of testing the following observations were made:

1. Fast settling particles, i. e, $\sim 300 \mu \mathrm{m}$ sand, transported out of the test tank readily and only accumulated during the first two to three cycles. All others cycles transported about the same amount of sand. [Figure 4-6 and Figure 4-7]

2. Very fast settling particles, i.e., $\sim 125 \mu \mathrm{m}$ stainless steel, transported readily but did accumulate until about the $3^{\text {rd }}$ to $4^{\text {th }}$ cycle, after which most of the additional solids introduced into the tank were transported out. [Figure 4-10 and Figure 4-13]

3. Solids did accumulate in the two "dead zones" of the tank, i.e., the locations opposite of the jet pump mixers and accumulations occurred almost immediately upon mixing the tank, i.e., before transfer began. [Example: Figure 4-20 and Figure 4-21]

4. The solids mounds that formed, referred to as the North and South mounds, were of unequal size with the North mound approximately three times the volume of the South mound. This volume difference was due to the different strengths for the two mixing jet pump velocities. That is, Jet Mixing Pump 2 was approximately $1 \mathrm{ft} / \mathrm{s}$ stronger than Jet Mixing Pump 1 [Figure 2-7]. This fact was demonstrated when a subsequent test was performed by rotating the pumps in the opposite direction, counter clockwise, resulting in the South mound becoming larger than the North mound. [Figure 4-74 and Figure 4-76]

5. Accumulated volumes determined by the two methods employed, i.e., laser and photographic, agreed on the average to within $22 \%$ for the North mound and $83 \%$ for the South mound. This measurement difference was not unexpected because the mounds were small, less than $1 \mathrm{inch}$ in height for the North mounds and less than $0.5 \mathrm{inch}$ in height for the South mounds. Furthermore, the small sized solids, i.e., gibbsite and zirconia, covered everything making the contrast between the supernatant and solids difficult for the photographic technique to clearly distinguish the liquid-solids interfaces. The smaller the volume and the poorer surface contrast the more measurement uncertainty for the photographic technique. [Table 4-5]

6. Core samples taken from the mounds clearly indicated that stainless steel is the predominant component of the bottom of the settled volumes. The composition gradually transitions to sand in the upper surface of the mounds. [Table 4-6 and Table 4-7]

7. At the end of 10 cycles for each of the two campaigns the amount of stainless steel that remained in the staging tank was $9.0 \%$ and $7.4 \%$, respectively.[Table 4-9 and Table 4-10]

8. In Campaign 2 , the $93 \%$ of the residual stainless steel was shown to be located within the mounds of accumulated solids with the remaining $7 \%$ in all other locations. [Table 4-8] 


\section{TABLE OF CONTENTS}

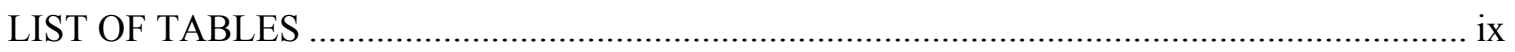

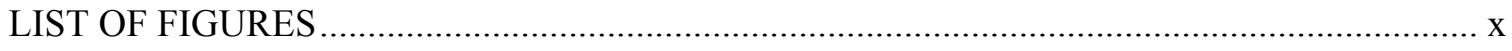

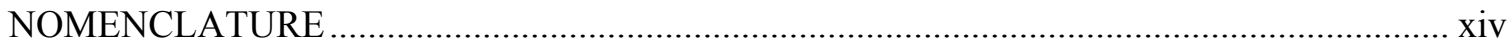

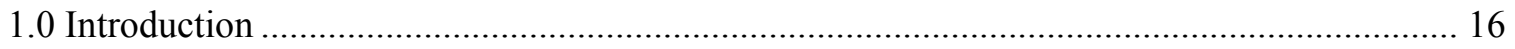

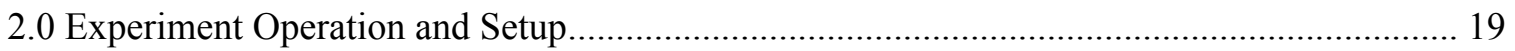

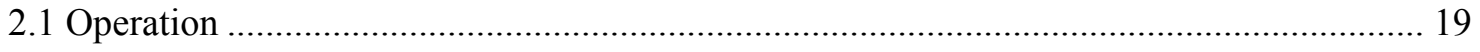

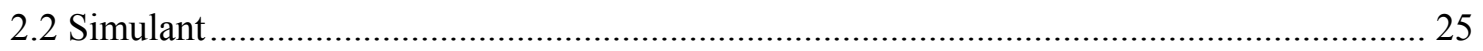

2.3 Utilization of the Measurement and Test Equipment .................................................... 28

3.0 Pre-Test Trial Runs and Measurement Techniques............................................................ 31

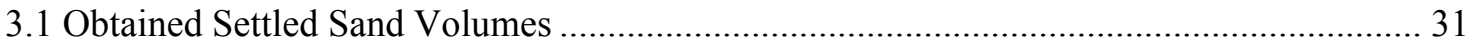

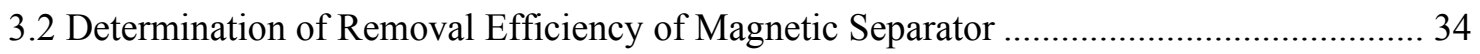

3.3 Determination of Jet Pump Flow Rate for Campaign 2 ................................................. 35

3.4 Overall Operation with Receipt Tanks, Magnetic Separator, and Solids Accumulation at

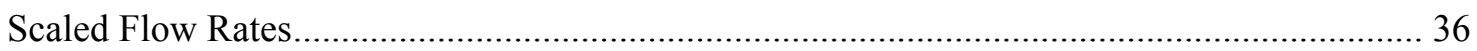

3.5 Wait Time between Each Feed Batch to Determine Effect on Accumulated Solids ........... 37

3.6 Feed Rate to the MDT that Will Not Disturb Accumulated Solids ...................................... 38

3.7 Position Accuracy of the Mechanical Positioner to Measure Solids Mound Heights and

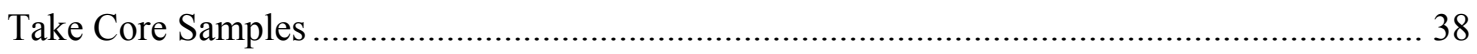

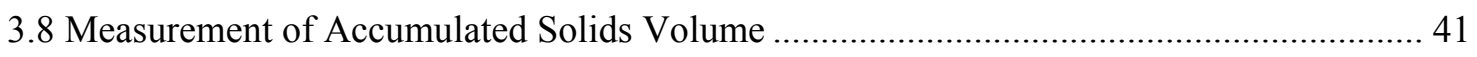

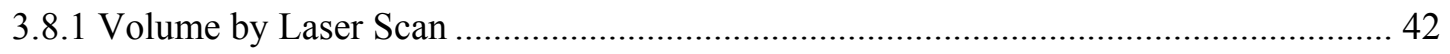

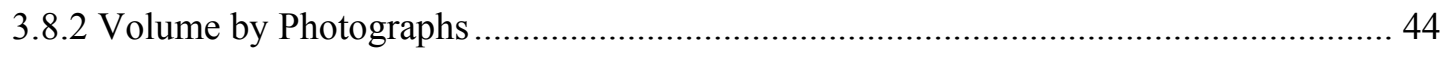

3.9 Core Sampler and Its Ability to Capture Complete cores............................................... 47

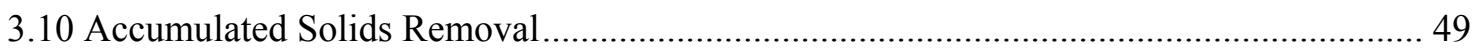

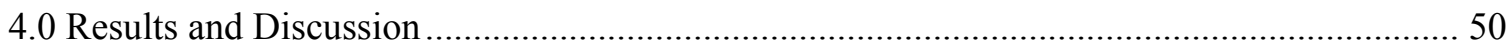

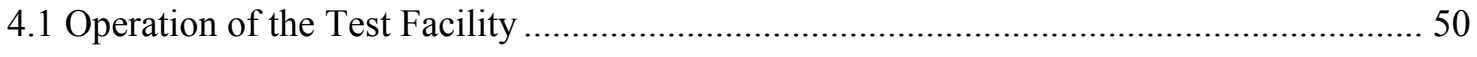

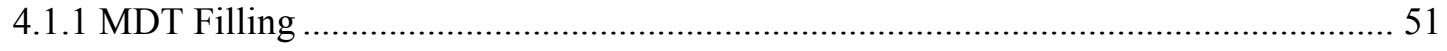

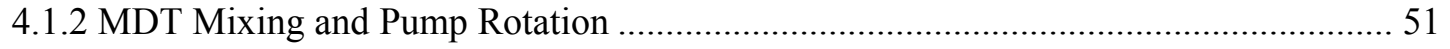

4.1.2.1 Mixing Jet Pump Fluctuation .................................................................................... 52

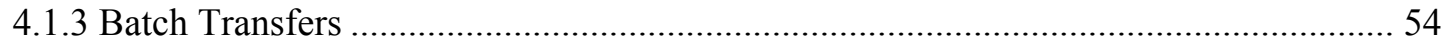

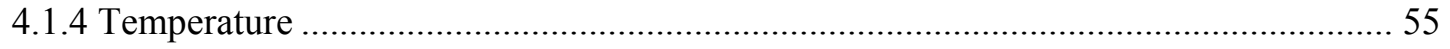

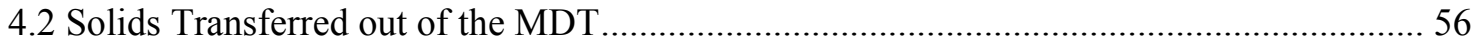




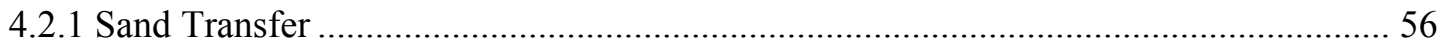

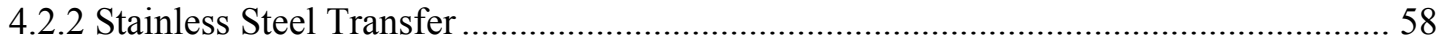

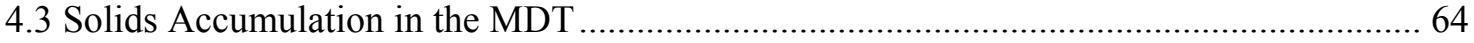

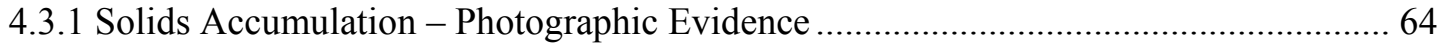

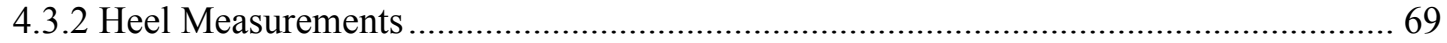

4.3.2.1 Determining Volume of the Accumulated Solids ....................................................... 70

4.3.2.1.1 Volume Estimation from Laser Measurements ...................................................... 70

4.3.2.1.2 Volume Estimation from Photographic Technique................................................. 91

4.3.2.1.3 Comparison of Volume Estimates: Laser versus Photographic Techniques........... 95

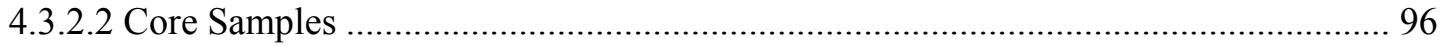

4.3.3 Accumulated Solids Captured after Cycle 10 of Each Campaign............................... 105

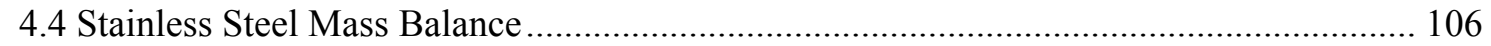

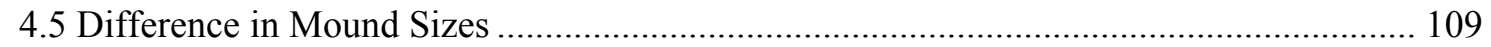

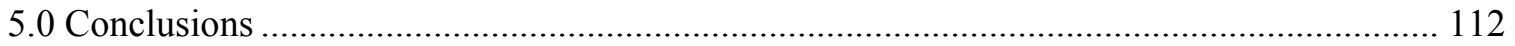

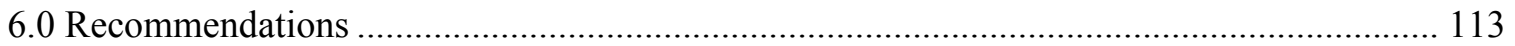

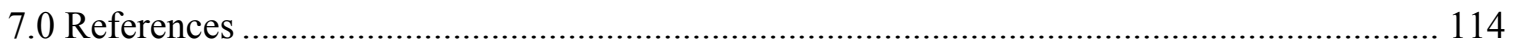

Appendix A: Particle Size Distribution for Simulant Solids ................................................. 115

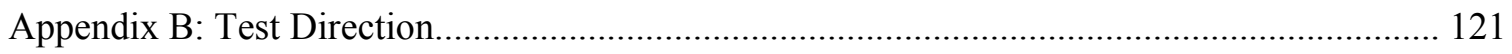

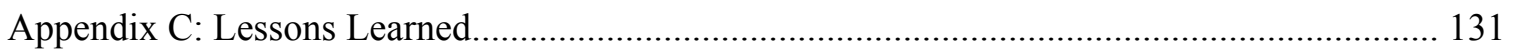

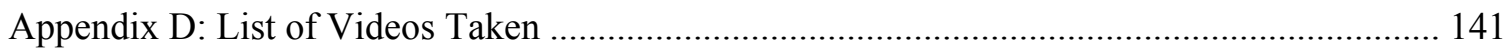




\section{LIST OF TABLES}

Table 1-1. Solids Accumulation Scouting Studies Test Objectives ........................................ 18

Table 2-1. Full Scale and Scaled Mixing Tank Dimension....................................................... 20

Table 2-2. Supernatant portion of SASS simulant used to suspend undissolved solids at $20^{\circ} \mathrm{C}$.. 26

Table 2-3. Typical Conceptual Simulant as Modified for SRNL Testing ................................... 26

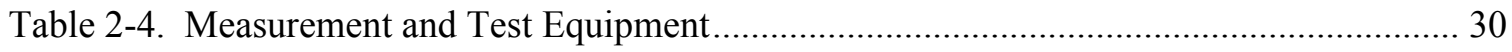

Table 3-1. Jet Pump Parameters used to determine Campaign 2 jet velocity................................ 35

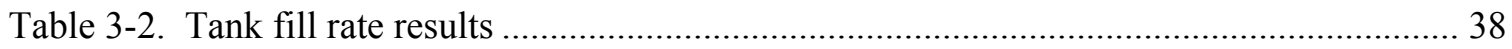

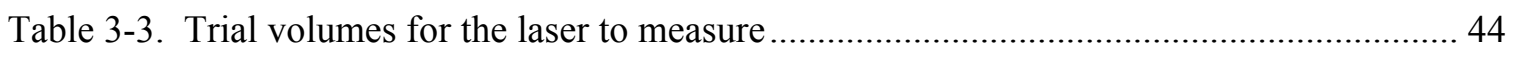

Table 4-1. Campaign 1 flowrates, velocities, and pump rotation............................................. 51

Table 4-2. Campaign 2 flowrates, velocities, and pump rotation.............................................. 52

Table 4-3. Stainless steel transferred during Campaign 1 ........................................................ 59

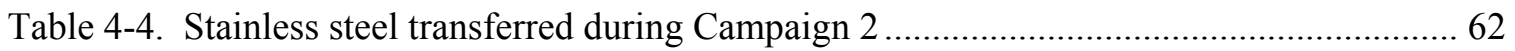

Table 4-5. Mound volumes of accumulated solids from both measurement techniques.............. 95

Table 4-6. Campaign 1 core sample stainless steel concentrations ............................................ 98

Table 4-7. Campaign 2 core sample stainless steel concentrations ........................................... 99

Table 4-8. Stainless steel recovered from Cycle 10 Heel after each campaign........................... 105

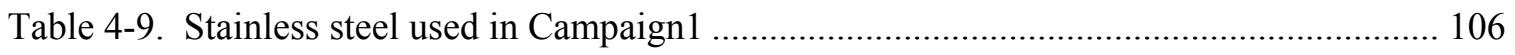

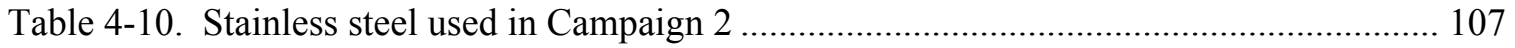




\section{LIST OF FIGURES}

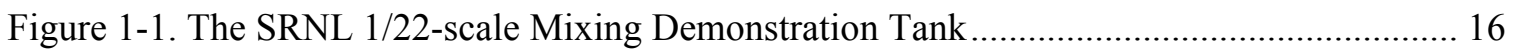

Figure 2-1. The P\&ID of the modified Mixing Demonstration Tank facility............................ 22

Figure 2-2. Conical-bottom feed tank showing agitator and drain port. ..................................... 24

Figure 2-3. Close up of feed tank drain shown in the preceding figure ..................................... 24

Figure 2-4. Particle sizes of SRNL simulant with four chosen UDS ........................................ 27

Figure 2-5. Settling characteristics of the SRNL simulant with four chosen UDS. .................... 27

Figure 2-6. Illustrating one of the mixing jet pumps (MJP) and its system to rotate pump........ 29

Figure 2-7. Top view of the MDT showing two MJPs and the angle convention........................ 29

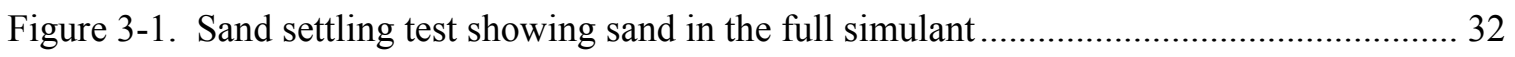

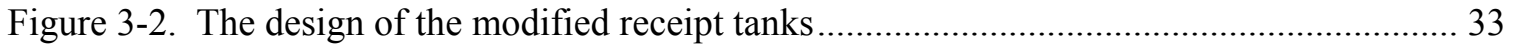

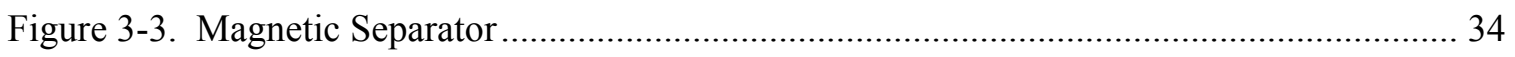

Figure 3-4. Pre-test solids accumulations during batch transfer to RT-1 …............................... 36

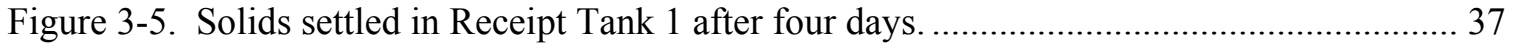

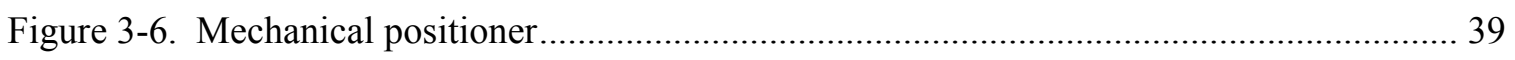

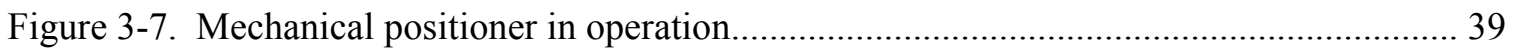

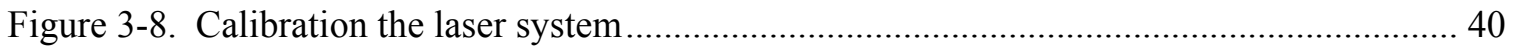

Figure 3-9. Positioner points being checked against full tank size grid map ............................. 41

Figure 3-10. Triangular platform that stood 3 inches above the tank bottom. ............................ 43

Figure 3-11. Determining volumes in the MDT with the laser using known volumes. ............... 44

Figure 3-12. Yellow sand mound in water to determine contrast between liquid and solids, ...... 45

Figure 3-13. Contour plot of the solids mound shown in the preceding figure........................... 45

Figure 3-14. Digital contour plot of the solids mound shown in the preceding figure ................ 46

Figure 3-15. Details of the core sampler (a) Illustrated drawing, (b) actual device.................... 48

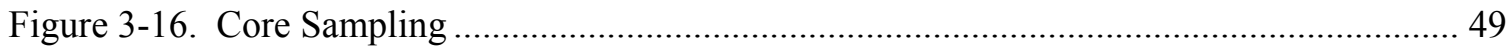

Figure 3-17. Hand-held magnet for separating stainless steel manually................................... 50

Figure 4-1. Example of the Jet Pump history during two batch transfers. .................................. 53

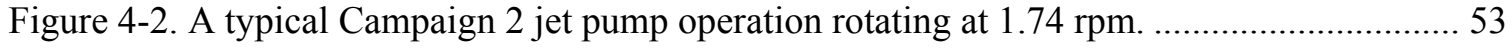


Figure 4-3. Example of the transfer pump and jet pump rotational histories. 54

Figure 4-4. Campaign 1 and 2 simulant temperatures in the MDT. 55

Figure 4-5. Receipt Tanks 1 and 2 showing sand transferred. 56

Figure 4-6. Sand transferred during Campaign 1. 57

Figure 4-7. Sand transferred during Campaign 2. 57

Figure 4-8. Batch to batch SS transferred during Campaign 1 60

Figure 4-9. Batch to batch SS transferred during Campaign 1 60

Figure 4-10. Shows the results of Figure 4-8 for each cycle. 61

Figure 4-11. Batch to batch SS transferred during Campaign 2 63

Figure 4-12. Batch to batch SS transferred during Campaign 2. 63

Figure 4-13. Shows the results of Figure 4-11 for each cycle. 64

Figure 4-14 (a) - (d). Accumulated solids footprints during Cycle 4 Batch Transfer. 66

Figure 4-15. Campaign 1 cycle to cycle "north mound" accumulated solids foot print. 67

Figure 4-16. Campaign 1 cycle to cycle "south mound" accumulated solids foot print 68

Figure 4-17. Campaign 2 cycle to cycle "north mound" accumulated solids foot print. 68

Figure 4-18. Campaign 2 cycle to cycle "south mound" accumulated solids foot print 69

Figure 4-19. Mound volumes obtain from laser measurements and showing $\pm 5 \%$ uncertainty... 70

Figure 4-20. Solids North mound after Cycle 1 of Campaign 1 73

Figure 4-21. Solids South mound after Cycle 1 of Campaign 1................................................. 73

Figure 4-22. North mound side view after Cycle 1 of Campaign 1 - Volume of $18.5 \mathrm{in}^{3} \ldots \ldots \ldots . . .74$

Figure 4-23. North mound top view after Cycle 1 of Campaign 1 - Volume of 18.5 in $^{3} \ldots \ldots \ldots . . .74$

Figure 4-24. South mound side view after Cycle 1 of Campaign 1 - Volume of 4.1 in $^{3} \ldots \ldots \ldots \ldots . . . . .75$

Figure 4-25. South mound top view after Cycle 1 of Campaign 1 - Volume of $4.1 \mathrm{in}^{3} \ldots \ldots \ldots \ldots . . . .75$

Figure 4-26. Solids North mound after Cycle 5 of Campaign 1................................................ 76

Figure 4-27. Solids South mound after Cycle 5 of Campaign 1............................................. 76

Figure 4-28. North mound side view after Cycle 5 of Campaign 1 - Volume of $37.1 \mathrm{in}^{3} \ldots \ldots \ldots . . .77$

Figure 4-29. North mound top view after Cycle 5 of Campaign 1 - Volume of $37.1 \mathrm{in}^{3} \ldots \ldots \ldots . . .77$

Figure 4-30. South mound side view after Cycle 5 of Campaign 1 - Volume of $14.2 \mathrm{in}^{3} \ldots \ldots \ldots . . . .78$

Figure 4-31. South mound top view after Cycle 5 of Campaign $1-$ Volume of 14.2 in $^{3} \ldots \ldots \ldots . . . .78$ 
Figure 4-32. Solids North mound after Cycle 10 of Campaign 1............................................. 79

Figure 4-33. Solids South mound after Cycle 10 of Campaign 1.............................................. 79

Figure 4-34. North mound side view after Cycle 10 of Campaign 1 - Volume of $37.9 \mathrm{in}^{3} \ldots \ldots . . .80$

Figure 4-35. North mound top view after Cycle 10 of Campaign 1 - Volume of 37.9 in $^{3}$.......... 80

Figure 4-36. South mound side view after Cycle 10 of Campaign $1-$ Volume of 13.0 in $^{3} \ldots \ldots . . .81$

Figure 4-37. South mound top view after Cycle 10 of Campaign $1-$ Volume of $13.0 \mathrm{in}^{3}$......... 81

Figure 4-38. Solids North mound after Cycle 1 of Campaign 2 2............................................... 82

Figure 4-39. Solids South mound after Cycle 1 of Campaign 2............................................... 82

Figure 4-40. North mound side view after Cycle 1 of Campaign 2 - Volume of $19.4 \mathrm{in}^{3}$.......... 83

Figure 4-41. North mound top view after Cycle 1 of Campaign 2 - Volume of $19.4 \mathrm{in}^{3} \ldots \ldots \ldots . . .83$

Figure 4-42. South mound side view after Cycle 1 of Campaign 2 - Volume of $2.0 \mathrm{in}^{3} \ldots \ldots \ldots . . . .84$

Figure 4-43. South mound top view after Cycle 1 of Campaign 2 - Volume of $2.0 \mathrm{in}^{3}$............. 84

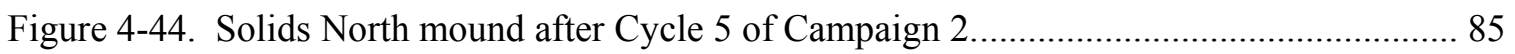

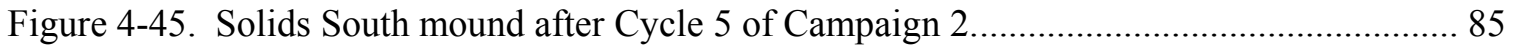

Figure 4-46. North mound side view after Cycle 5 of Campaign 2 - Volume of $30.9 \mathrm{in}^{3}$.......... 86

Figure 4-47. North mound top view after Cycle 5 of Campaign 2 - Volume of 30.9 in $^{3}$........... 86

Figure 4-48. South mound side view after Cycle 5 of Campaign 2 - Volume of $6.4 \mathrm{in}^{3} \ldots \ldots \ldots \ldots . . . . .87$

Figure 4-49. South mound top view after Cycle 5 of Campaign 2 - Volume of 6.4 in $^{3} \ldots \ldots \ldots \ldots . . .87$

Figure 4-50. Solids North mound after Cycle 10 of Campaign 2 ............................................ 88

Figure 4-51. Solids South mound after Cycle 10 of Campaign 2 2............................................. 88

Figure 4-52. North mound side view after Cycle 10 of Campaign 2 - Volume of 35.4 in $^{3}$........ 89

Figure 4-53. North mound top view after Cycle 10 of Campaign 2 - Volume of 35.4 in $^{3}$......... 89

Figure 4-54. South mound side view after Cycle 10 of Campaign 2 - Volume of $9.2 \mathrm{in}^{3} \ldots \ldots \ldots . . .90$

Figure 4-55. South mound top view after Cycle 10 of Campaign 2 - Volume of $9.2 \mathrm{in}^{3} \ldots \ldots \ldots . . .90$

Figure 4-56. Mound volumes obtain from photographic measurements and showing $\pm 20 \%$...... 91

Figure 4-57. Photographic technique: Cycle 10 of Campaign 2 - North Mound Top View........ 92

Figure 4-58. Photographs of changing tank level to estimate North mound volume: (a) to (g)... 93

Figure 4-59. Photographic technique: Cycle 10 of Campaign 2 - South Mound Top View........ 94

Figure 4-60. Photographs of changing tank level to estimate South mound volume: (a) to (c)... 94 
Figure 4-61. Campaign 2, core sample 20-2 taken from the North mound, ,............................. 96

Figure 4-62. Campaign 1 core sample 5-6, Table 4-6, taken from the North mound, ................. 97

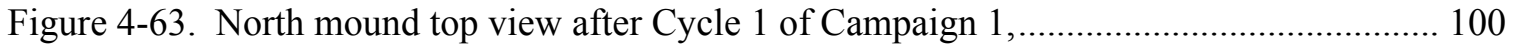

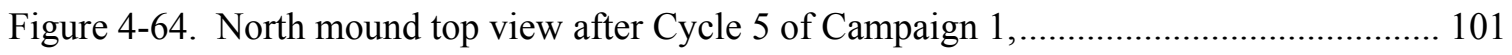

Figure 4-65. North mound top view after Cycle 10 of Campaign 1,....................................... 101

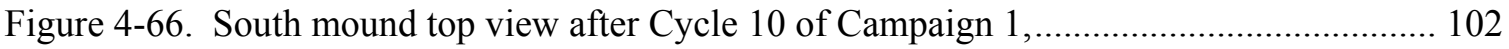

Figure 4-67. North mound top view after Cycle 1 of Campaign 2,....................................... 102

Figure 4-68. North mound top view after Cycle 5 of Campaign 2,....................................... 103

Figure 4-69. North mound top view after Cycle 10 of Campaign 2,..................................... 103

Figure 4-70. South mound top view after Cycle 10 of Campaign 2, ........................................ 104

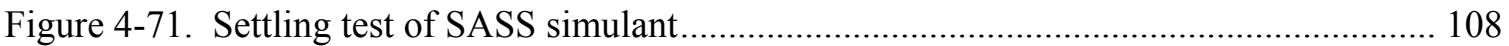

Figure 4-72. MDT showing clockwise rotation of jet pump mixings and influence on mounds. 109 Figure 4-73. North mound side view with counter clockwise rotation of jet pumps ................. 110

Figure 4-74. North mound top view with counter clockwise rotation of jet pumps................... 110

Figure 4-75. South mound side view with counter clockwise rotation of jet pumps ................. 111

Figure 4-76. South mound top view with counter clockwise rotation of jet pumps................... 111

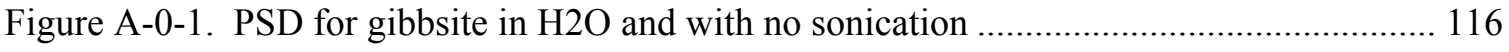

Figure A-0-2. PSD for zirconium oxide in $\mathrm{H} 2 \mathrm{O}$ and with no sonication................................. 117

Figure A-0-3. PSD for yellow sand in $\mathrm{H} 2 \mathrm{O}$ and with no sonication .................................... 118

Figure A-0-4. PSD for stainless steel in 50\% isopropyl no sonication .................................... 119

Figure A-0-5. PSD for stainless steel in 50\% isopropyl and with sonication............................. 120

Figure C-0-1 Moyno Pump Used (from Moyno.com Website). ................................................. 132

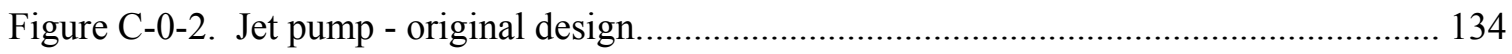

Figure C-0-3. Jet pump - new, but not implemented, design. ................................................ 136

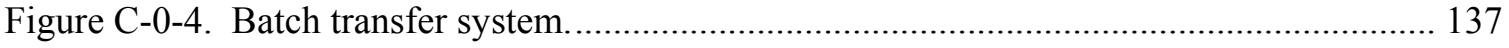




\section{NOMENCLATURE}

$A_{i}$

Ar

Campaign Completion of 10 complete cycles done at a single jet pump flow rate

Cycle One complete fill and empty of the MDT

d

$\rho_{\mathrm{L}}$

$\rho_{\mathrm{S}}$

DNFSB

DST

EDL

ERPS

FB

FT

g

JHA

Heel

HAP

HLW

LSIT

MDT

MJP

PTF

RT

SASS

SRNL

The ith area element used to determine mound volumes [Eqs (1), (2), (3)]

Archimedes Number, Eq. (1)

Particle Diameter

Density of Supernatant

Density of Solid Particles

Defense Nuclear Facilities Safety Board

Double-Shell Tank

Engineering Development Laboratory (Performing Organization)

E\&CPT Research Programs Section [E\&CPT=Environmental \& Chemical Process Technology]

Feed Batch (to WTP) - Approximately 145,000 gallons - 6.5 FB constitute one transferrable Hanford feed tank volume

Feed Tank - Where simulant will be prepared and fed to the MDT

Gravitational Constant

Job Hazards Analysis

In the MDT the heel refers to the simulant at the bottom of the tank that is not transferred after each fill and empty cycle is complete. For SASS, it is estimated to be 3.23 inches in height and contains both liquid and accumulated solids. This scaled height is equivalent to the minimum DST mixer pump operating level of 72 inches.

Hazards Assessment Package

High (Radioactive) Level Waste

Large Scale Integrated Testing Program

Mixing Demonstration Tank

Mixing Jet Pump [MJP1= Pump 1 and MJP2 = Pump 2]

Pretreatment Facility of WTP

Receipt Tank - There are 7 receipt tanks, 6 will receive one Feed Batch (13.1 gal) and 1 will receive a half-Feed Batch (6.9 gal)

Solids Accumulation Scouting Studies (This task)

Savannah River National Laboratory 
Solids Accumulation Scouting Studies

SRNL-STI-2012-00508

Revision 0

SS

Stainless Steel

SSMD

Small Scale Mixing Demonstration

UDS

Undissolved Solids

V

Volume of solids mounds

$v$

Kinematic Viscosity

WFD

Waste Feed Delivery

WTP

Waste Treatment \& Immobilization Plant

SRNL

Savannah River National Laboratory

$Z_{i}$

The ith element of height in determine mound volumes [Eqs (1), (2), (3)] 


\subsection{Introduction}

The overall objective of Solids Accumulation Scouting Studies (SASS) activities was to perform scaled testing to understand the behavior of remaining solids in a Double Shell Tank (DST) at Hanford during multiple fill, mix, and transfer operations that are typical of the High Level Waste (HLW) feed delivery mission [1]. As described in the Task Technical and Quality Assurance Plan [2], testing focused on accumulation of total solids over time and the propensity for simulated fissile material to concentrate over time.

The overall Test Plan [3] of the Mixing and Sampling Demonstration Project [4] includes two scaled test platforms to evaluate the baseline design for mixing and transferring slurry from DST 241-AY-102, the first staged HLW feed, to the Waste Treatment and Immobilization Plant (WTP). However, 241-AW-105, the second staged HLW tank, is being modeled for this test scope because current waste feed delivery planning shows it being utilized more than any other of the waste feed tanks over the life of the waste-treatment mission. The small scale test was performed at SRNL's 1:22-scale Mixing Demonstration Tank (MDT), Figure 1-1, to perform mixing and transfer studies.

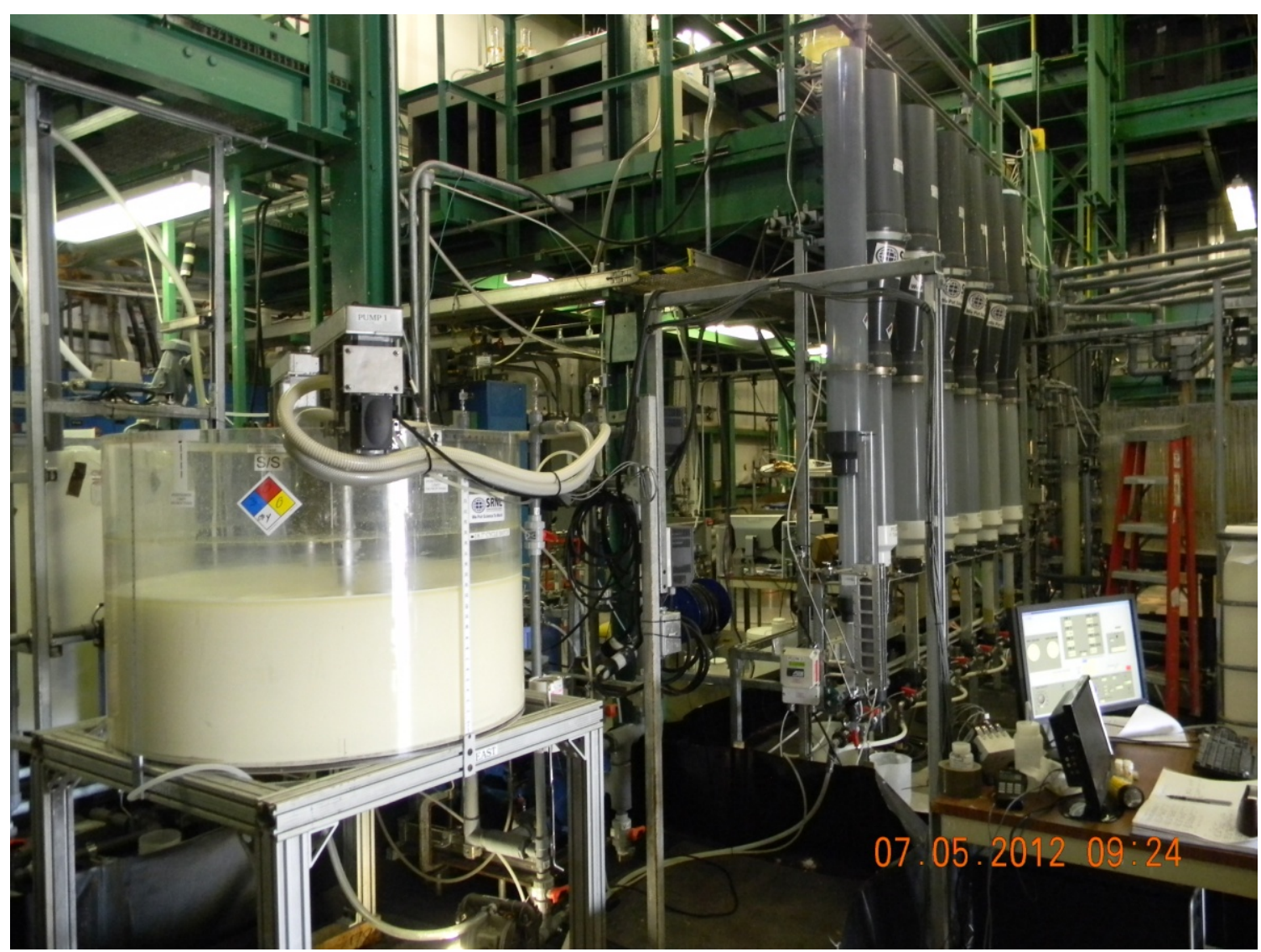

Figure 1-1. The SRNL 1/22-scale Mixing Demonstration Tank with the 7 batch receipt tanks. 
Actually the MDT system was modified from past mixing tests [5-7]. The principal changes were:

- The 22 air lift circulating tubes were removed from the MDT; they exist in the AY-102 tank but not in the AW-105 tank

- A central tank drain was installed to better empty the MDT during heel measurements

- The number of receipt tanks to receive tank transfer was increased from six to seven because of the last "half" batch that was transferred before reaching the heel level

- The volume of the bottom section of the receipt tanks was made smaller due to the reduced solids loading, i.e., $100 \mathrm{~g} / \mathrm{l}$, for this task

- A preparation feed system was created because of the many fill and empty cycles, which required much more simulant that had to be made on a daily bases

- A magnetic separator was added into the transfer line to the receipt tanks to separate stainless steel solids

- The transfer pump, Pump 3, was turned upside down so that the discharge port was on the top to minimize slurry plugs.

The specific objectives of the SRNL test, see Table 1-1, referred as SASS, included performing a series of Waste Feed Delivery (WFD) to WTP transfer and refill operations using the 1:22-scale MDT and evaluate the bulk material that remains in the tank. Testing determined the concentration and distribution of the fastest settling particles that accumulate in the tank heel. Providing insight into how very fast settling particles are distributed in a WFD feed staging tank is essential to criticality evaluations that include the accumulation of dense plutonium and uranium containing solids. To represent these fast settling solids stainless steel particles were utilized. The scope of the work is limited to preliminary scoping studies, the results of which will be used to define larger scale test work; to be performed using the test platform at Hanford.

Concurrent with this activity was the selection of appropriately complex simulants that are integrated with WTP simulant selection and supported by accurate analytical techniques to characterize the material of interest. This includes using simulants characteristic of the Hanford tank waste and following the recommended guidelines in Lee et al. [8]. However, the exact simulant used for SASS is listed in a simulant development plan [9] that was agreed to by the overall test program management. Finally, the test also included sampling techniques [9] for characterizing the residual tank waste solids that accumulate in the tank after a series of transfer and refill operations.

As stated, a DST transfer campaign included a series of fill and transfer operations with the MDT. A transfer operation was completed when six and one-half batches of slurry were transferred from the MDT to seven receipt tanks. The number of batch transfers is primarily based on full-scale operation. The volume of each batch is based on Interface Control Document-19 [10] which requires full scale batches of 145,000 gallons to be sent to WTP. Since AW-105 has approximately 946,000 gallons of transferrable waste then the total number of batches is approximately 6.5. The full tank volume of AW-105, i.e., 1,144,000 gallons, is not transferrable because a minimum volume, referred to as a heel, is needed to maximize mixer pump operations. Following a successful transfer operation, the amount stainless steel solids transferred out of the MDT were measured and then additional simulant was added to refill the mixing tank. A series of 10 transfer and refill operations were performed in a campaign. The stainless steel solids remaining in the tank after each transfer campaign were measured and compared to the total stainless steel solids that are added during testing. 
The test objectives are summarized in Table 1-1 as listed in the overall test plan [3].

Table 1-1. Solids Accumulation Scouting Studies Test Objectives

\begin{tabular}{|c|c|c|}
\hline Objective & Success Criteria & Result \\
\hline \multirow[t]{6}{*}{$\begin{array}{l}\text { Demonstrate at two jet } \\
\text { nozzle velocities the } \\
\text { potential accumulation of } \\
\text { solids in the DST after } \\
\text { several transfer and re-fill } \\
\text { operations are conducted. }\end{array}$} & $\begin{array}{l}\text { Mixing and transfer demonstrations are } \\
\text { performed at two different jet nozzle } \\
\text { velocities with a base simulant that } \\
\text { contains moderately sized (approximately } \\
100 \text { microns), dense particles to represent } \\
\text { fissile material in the Hanford tank waste. } \\
\text { The spike particles are distinguishable in } \\
\text { collected samples by a physical property } \\
\text { that can be exploited for quantification. }\end{array}$ & $\begin{array}{l}\text { Two Velocities were used } 22.9 \\
\mathrm{ft} / \mathrm{s} \text {, which was used in } \\
\text { previous MDT testing for the } \\
\text { minimum energy level and } 23.9 \\
\mathrm{ft} / \mathrm{s} \text { which was the minimum } \\
\text { velocity that allowed solids to } \\
\text { accumulated in the mixing } \\
\text { tank. }\end{array}$ \\
\hline & $\begin{array}{l}\text { The dense } 100 \text { micron particles that } \\
\text { represent the very fast settling particles } \\
\text { that can accumulate inside a DST used } \\
\text { for several staged feeds are quantified } \\
\text { relative to the amount of the solids added } \\
\text { to the tank. }\end{array}$ & $\begin{array}{l}\text { Particle of } 125 \text { micron were } \\
\text { used. }\end{array}$ \\
\hline & $\begin{array}{l}\text { The relative quantities of undissolved } \\
\text { solids in each transfer batch are } \\
\text { estimated. }\end{array}$ & $\begin{array}{l}\text { Relative quantities of fast } \\
\text { settling particles, sand, were } \\
\text { estimated. }\end{array}$ \\
\hline & $\begin{array}{l}\text { The accumulation of heel solids is } \\
\text { evaluated after each tank volume transfer. } \\
\text { Visual changes will be noted and } \\
\text { photographs will be taken. }\end{array}$ & $\begin{array}{l}\text { Photographs of the } \\
\text { accumulated solids foot print } \\
\text { were obtained for each } \\
\text { transfer. }\end{array}$ \\
\hline & $\begin{array}{l}\text { The accumulation of heel solids is } \\
\text { quantified after the } 1^{\text {st }}, 5^{\text {th }} \text { and } 10^{\text {th }} \text { tank } \\
\text { volume transfer by measuring the volume } \\
\text { of heel in the tank. In the case where } 10 \\
\text { transfer volumes are not performed } \\
\text { accumulation of heels solids is quantified } \\
\text { at the } 1^{\text {st }}, 5^{\text {th }} \text {, and last cycles. }\end{array}$ & $\begin{array}{l}\text { Heel measurements were } \\
\text { performed after the } 1 \mathrm{st}, 5 \text { th, } \\
\text { and 10th tank volume for both } \\
\text { Campaigns } 1 \text { and } 2 .\end{array}$ \\
\hline & $\begin{array}{l}\text { Correlations relating the fraction of the } \\
\text { mock very fast settling solids transferred } \\
\text { and remaining in the tank are evaluated } \\
\text { with respect to each transfer batch and } \\
\text { after multiple tank volume transfers. }\end{array}$ & Correlations were developed. \\
\hline \multirow{2}{*}{$\begin{array}{l}\text { Develop and demonstrate } \\
\text { quantification techniques to } \\
\text { characterize the residual } \\
\text { tank waste in-situ. }\end{array}$} & $\begin{array}{l}\text { Techniques to sample and quantify the } \\
\text { volume of residual solids are identified } \\
\text { and documented. }\end{array}$ & $\begin{array}{l}\text { Techniques were developed, } \\
\text { demonstrated, and the results } \\
\text { are discussed. }\end{array}$ \\
\hline & $\begin{array}{l}\text { The error in the heel volume measurement } \\
\text { is quantified. }\end{array}$ & $\begin{array}{l}\text { Error was quantified and is } \\
\text { discussed. }\end{array}$ \\
\hline
\end{tabular}




\subsection{Experiment Operation and Setup}

The test operation was done to mimic the process expected with the Hanford Tank Farm to feed the Waste Treatment and Immobilization Plant (WTP). The staging tank for this task is AW-105, will be repeatedly filled with select wastes to be processed and then for each filled tank the contents will be transferred to WTP in batches, Table 2-1, currently planned to be divided into approximately 6.5 batches, until only a heel of 72 -inches of remained. At that time the staging tank will be refilled to repeat the process.

The following describes the scaled process and equipment used to mimic the feeding process. Also discussed is the simulant employed to represent the settling characteristics of the real waste and measurement equipment to quantify many aspects of the operation.

\subsection{Operation}

The two principal parts of the SASS test were the simulant and the test facility. They will be explained in more detail shortly, but it is important to understand the overall test operation to better understand the individual parts. The explanation of the operation here is only a summary of the overall operation. See Appendix B: Test Direction, for more detail. Refer to Figure 2-1, the piping and instrument diagram, for the equipment explanations below and Table 2-1 for the simulant test volumes.

1. The feed tank, shown above the Mixing Demonstration Tank (MDT) in Figure 2-1(a), was filled with about $80 \%$ of the test supernatant and $100 \%$ of the test solids. It was thoroughly mixed and then transferred to the MDT at approximately $1.75 \mathrm{gpm}$, but the MDT was not mixing during a fill.

2. The remaining $20 \%$ of the supernatant was then added to the feed tank to clean out the remaining solids and transferred to the MDT until it was filled to the full volume of 103.6 gallons, which was 18.7 inches in tank height. This filling generally occurred at the end of a day so the full MDT was left for about 12 hours before it was mixed. (See the Note on fully transferring the solids from the feed tank to the MDT at the end of this list of operational steps about concerns to transfer all the solids to the MDT.)

3. The following morning would be to mix the MDT at a predetermined jet pump velocity and rotational speed. For Campaign 1 the target velocity was $22.4 \mathrm{ft} / \mathrm{s}$ and $1.57 \mathrm{rpm}$ and for Campaign 2 it was $23.5 \mathrm{ft} / \mathrm{s}$ and $1.72 \mathrm{rpm}$. The MDT was mixed for a minimum of the time necessary to turn over the tank volume 5 times. For example, in Campaign 1 the $22.4 \mathrm{ft} / \mathrm{s}$ required $8 \mathrm{gpm}$ from each of the two jet pumps, therefore, the minimum mixing time was 103.6 gal x $5=518.1$ gal giving a time of $518.1 \mathrm{gal} /(2 \times 8 \mathrm{gpm})=32$ minutes and 23 seconds. (Note, 103.6 gal was a full cycle batch equal to 392 liters.)

4. When the MDT was fully mixed, the first batch was ready for transfer. First, photographs were taken of the bottom of the MDT to record the footprints of the settled solids. Then the temperature of the MDT volume was recorded. Finally, transfer began which was directed through the magnetic separator and into the appropriate receipt tank at a flowrate of $0.58 \mathrm{gpm}$.

5. Once a full batch of 13.1 gallons was transferred, the transfer pump was stopped and the mixing in the MDT was stopped.

6. After 20 minutes the heights of the settled solids in the target Receipt Tank were measured. Usually, only the sand height was visible. During these 20 minutes the 
Magnetic Separator was made ready for the next transfer by flushing it of the stainless steel solids that were captured to be measured.

Table 2-1. Full Scale and Scaled Mixing Tank Dimension

\begin{tabular}{|c|c|c|c|}
\hline Parameter & Unit & Full-Scale (1) & Scaled (2) \\
\hline Tank Diameter & inch & 900 & 40.5 \\
\hline Scale Factor & - & $1: 1$ & $1: 22$ \\
\hline Bottom Geometry & - & Flat & Flat \\
\hline Fill Volume & gallon & $\sim 1144000$ & 103.6 \\
\hline Tank Operating Height & inch & 416 & 18.7 \\
\hline Transfer Batches 1 to 6 & gallon & $\sim 145000$ & 13.13 \\
\hline Transfer Batch 7 & gallon & $\sim 76000$ & 6.88 \\
\hline Tank Heel & gallon & $\sim 198000$ & 17.93 \\
\hline Tank Heel Height & inch & 72 & 3.23 \\
\hline MJPs Location from Center (3) & inch & 240 & 11.9 \\
\hline MJP Suction Elevation (4) & inch & $5 \pm 1$ & 0.23 \\
\hline MJP Nozzle Diameter & inch & 6 & 0.27 \\
\hline MJP Nozzle Elevation (4) & inch & 18 & 0.41 \\
\hline MJP Rotational Speed (5) & $\mathrm{rpm}$ & 0.2 & $1.57 \& 1.72$ \\
\hline Nozzle Exit Velocity (5) & $\mathrm{ft} / \mathrm{sec}$ & 60 & $22.4 \& 23.5$ \\
\hline TP Location from Center (6) & inch & 36 & 3.5 \\
\hline TP Suction Inlet Diameter (7) & inch & $2.25-2.40$ & 0.245 \\
\hline TP Suction Inlet Height & inch & 6 & 0.27 \\
\hline TP Line Diameter & inch & 3.07 & 0.18 \\
\hline Transfer Velocity (8) & $\mathrm{ft} / \mathrm{sec}$ & $\sim 6$ & $7.3(0.58 \mathrm{gpm})$ \\
\hline Tank Obstructions & - & None & None \\
\hline \multicolumn{4}{|l|}{ Notes: } \\
\hline \multicolumn{4}{|c|}{ MJP = Mixing Jet Pump, TP = Transfer Pump } \\
\hline \multicolumn{4}{|c|}{ (1) Full Scale Tank = 241-AW-105 at Hanford } \\
\hline \multicolumn{4}{|c|}{ (2) Scaled = Mixing Demonstration Tank at SRNL } \\
\hline \multicolumn{4}{|c|}{ (3) MJPs are equal distances from center. The scaled 11.9 in. was larger } \\
\hline \multicolumn{4}{|c|}{ than the $1: 22$ scale of $10.9 \mathrm{in}$. because existing was used to reduce project cost. } \\
\hline \multicolumn{4}{|c|}{\begin{tabular}{|l|l|l|} 
(4) Elevation are from tank bottom & \\
\end{tabular}} \\
\hline \multicolumn{4}{|c|}{ (5) Two scaled MJP rotational speeds and nozzle velocities } \\
\hline \multicolumn{4}{|c|}{ are based on scale factor (1/22) and scale factor exponent, } \\
\hline \multicolumn{4}{|c|}{0.32 for Campaign 1 and 0.30 for Campaign 2.} \\
\hline \multicolumn{4}{|c|}{ (6) A single line through the tank center bisects both the } \\
\hline \multicolumn{4}{|c|}{\begin{tabular}{l|l|l} 
MJPs and the Transfer Line & \\
\end{tabular}} \\
\hline \multicolumn{4}{|c|}{ (7) Full-scale diameter is under development } \\
\hline \multicolumn{4}{|c|}{ (8) The velocity in transfer line from MDT to TP was $3.9 \mathrm{ft} / \mathrm{s}$ because inlet } \\
\hline \multicolumn{4}{|c|}{ diameter was 0.245 inch. From the pump discharge to a receipt tank the } \\
\hline \multicolumn{4}{|c|}{ velocity increased to $7.3 \mathrm{ft} / \mathrm{s}$ due to the smaller diameter transfer line. } \\
\hline
\end{tabular}




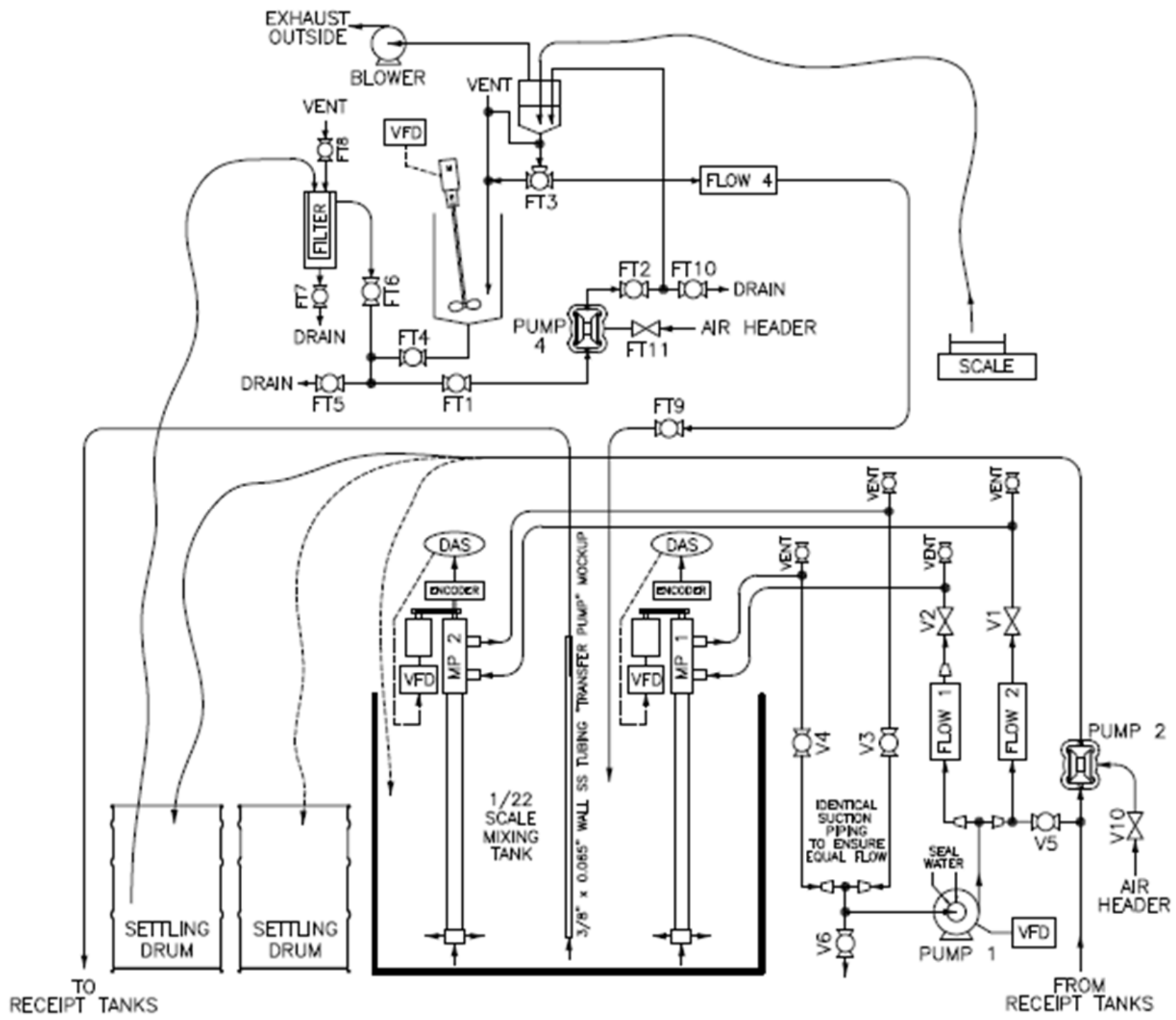

(a) Process Feed Preparation and Mixing Systems 


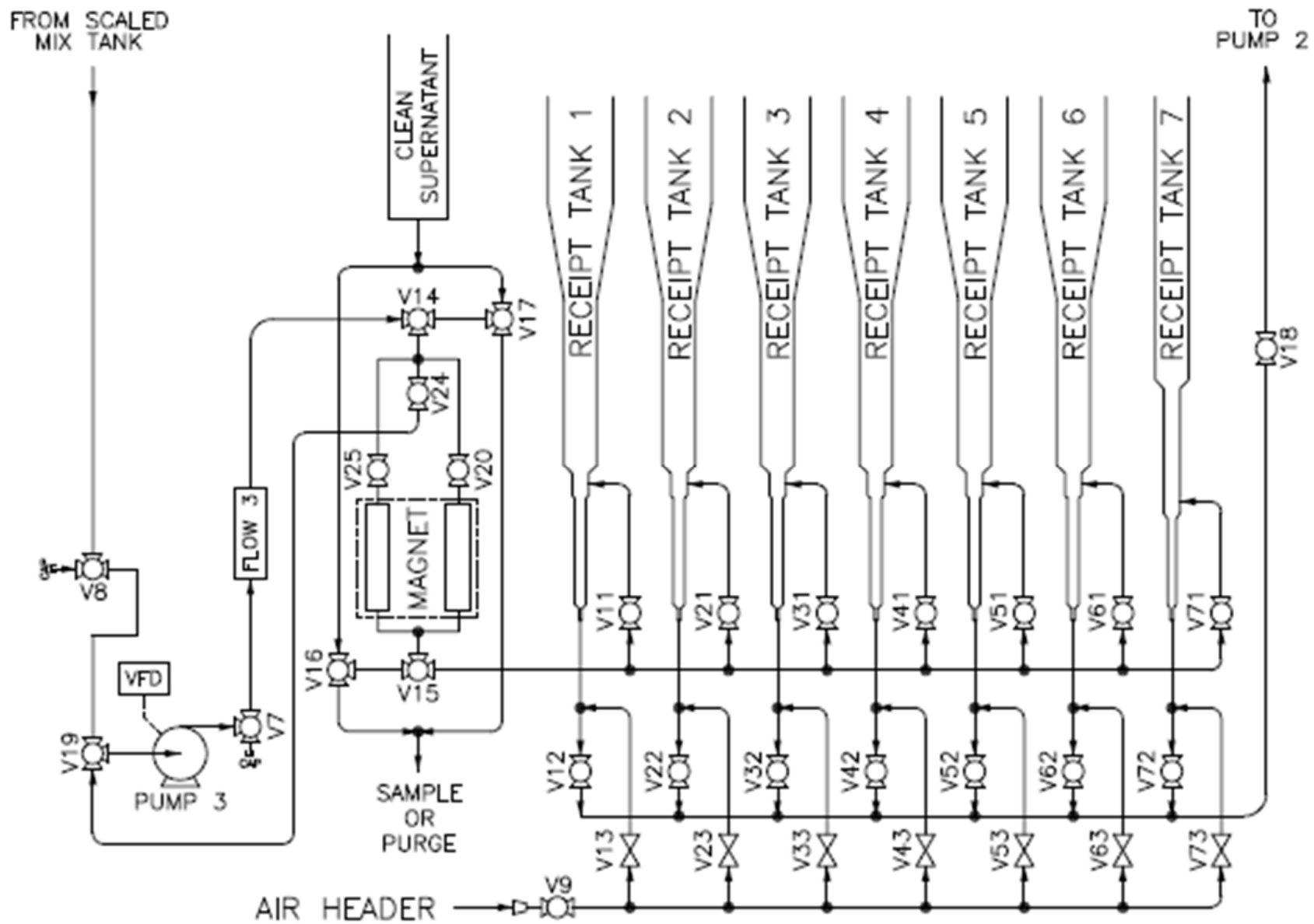

(b) Process Transfer and Receipt Systems

Figure 2-1. The P\&ID of the modified Mixing Demonstration Tank facility.

7. Once the sand height was recorded (Step 6) the mixing in the MDT began again and a time period was waited sufficient to turn over the current volume five times. Due to the smaller volume after each transfer this mixing period became successively shorter.

8. Steps 4 through 7 were repeated for transfer batches 2 to 6 .

9. The seventh and last batch was only a partial batch of 6.9 gal., because a heel of 3.23 inches was left in the MDT to represent the 72-inch heel to be left in the full scale tank.

10. Once the heel was reached the cycle was complete and that transfer was stopped. Simultaneously, while operating the cycle, the feed tank was filled with another charge of simulant to be ready for the next cycle. Note that cycle batches for the second through the tenth cycles are only 85.7 gallons each i.e., not 103.6 gallons, because of the heel of 17.9 gal., which remained in the MDT.

11. At the end of the $1^{\text {st }}, 5^{\text {th }}$, and $10^{\text {th }}$ cycles the following day was dedicated to making heel measurements to evaluate the accumulated solids. The method entailed:

a. Mix the 17.9 gal. of heel in the MDT to suspend the gibbsite and zirconia solids.

b. Stop the rotation of the jet pumps such that they were pointing at each other, that is, away from the accumulated solids mounds, and reduce the jet pump flowrate 
to a rate that appeared to keep the small solids suspended but did not disturb the accumulated solids ( $\sim 3 \mathrm{gpm} / \mathrm{pump}$ ).

c. Drain the MDT of the moving slurry. An arbitrary rate of $\sim 0.8$ gpm was used. As soon as the accumulated solid mounds become visible make note of the tank level.

d. When drained, begin mapping the tank bottom with laser system.

e. When the mapping sequence is complete use the laser positioning system to locate the core sampler. Core samples were only taken from the North mound (the largest) after the $1^{\text {st }}$ and $5^{\text {th }}$ cycles. After the $10^{\text {th }}$ cycle core samples were taken from both mounds. (See Section 4.9, Heel Measurement, for an explanation of this method.)

f. Once core sampling is complete begin to slowly refill the MDT with the removed heel material in 0.2-inch increments. At each increment pictures of each of the mounds are taken.

g. Once both mounds are covered the filling continued until the MDT heel is fully re-established to 3.23 inches.

h. The heel measurement process is now complete and the MDT is now ready to be fully refilled to begin the next cycle.

Note on fully transferring the solids from the feed tank to the MDT:

By design some of the solids were fast settling; therefore, how the simulant was prepared and transferred was important. Some of the important features for completely transferring the solids were:

- Conical bottom feed tank, Figure 2-2 - To make sure all the solids left the feed tank the bottom was conical to assist in draining. Nonetheless, the sand and stainless steel (SS) did accumulate on the sloping bottom and needed flushing to insure a total transfer.

- Smooth transitions from feed tank cone to the drain line, Figure 2-3 - Unless special fittings are ordered, the standard pipe fittings always have areas that allow liquid and solids to collect even when a tank is fully drained. Therefore, the drain of the bottom of the feed tank was cut and sanded to match smoothly with the pipe flange so there would be no hold-up areas. That is, the transition from the cone to the drain pipe was continuous and smooth.

- Double agitation - The mixing of the feed tank was performed with two devices: A double diaphragm positive displacement pump and a standard agitator. The function of the agitator is straight forward in that it kept the simulant in the tank well mixed during preparations and transferring. The pump had several functions: It circulated the simulant from the bottom of the feed tank to solids receipt tank, which then drained back to the feed tank, It initiated flow when solids settled in the tank and re-suspended those solids. That is, the pump was the primary mixer when the simulant level fell below the agitator. It also filled the feed tank with supernatant from settling drums through the filter to initiate feed preparation. (The solids receipt tank, above the feed tank, was not necessary and it was not used for its designed function to add solids to the system. They were added directly into the top of the feed tank, which was found to be a faster method after the test began.) 


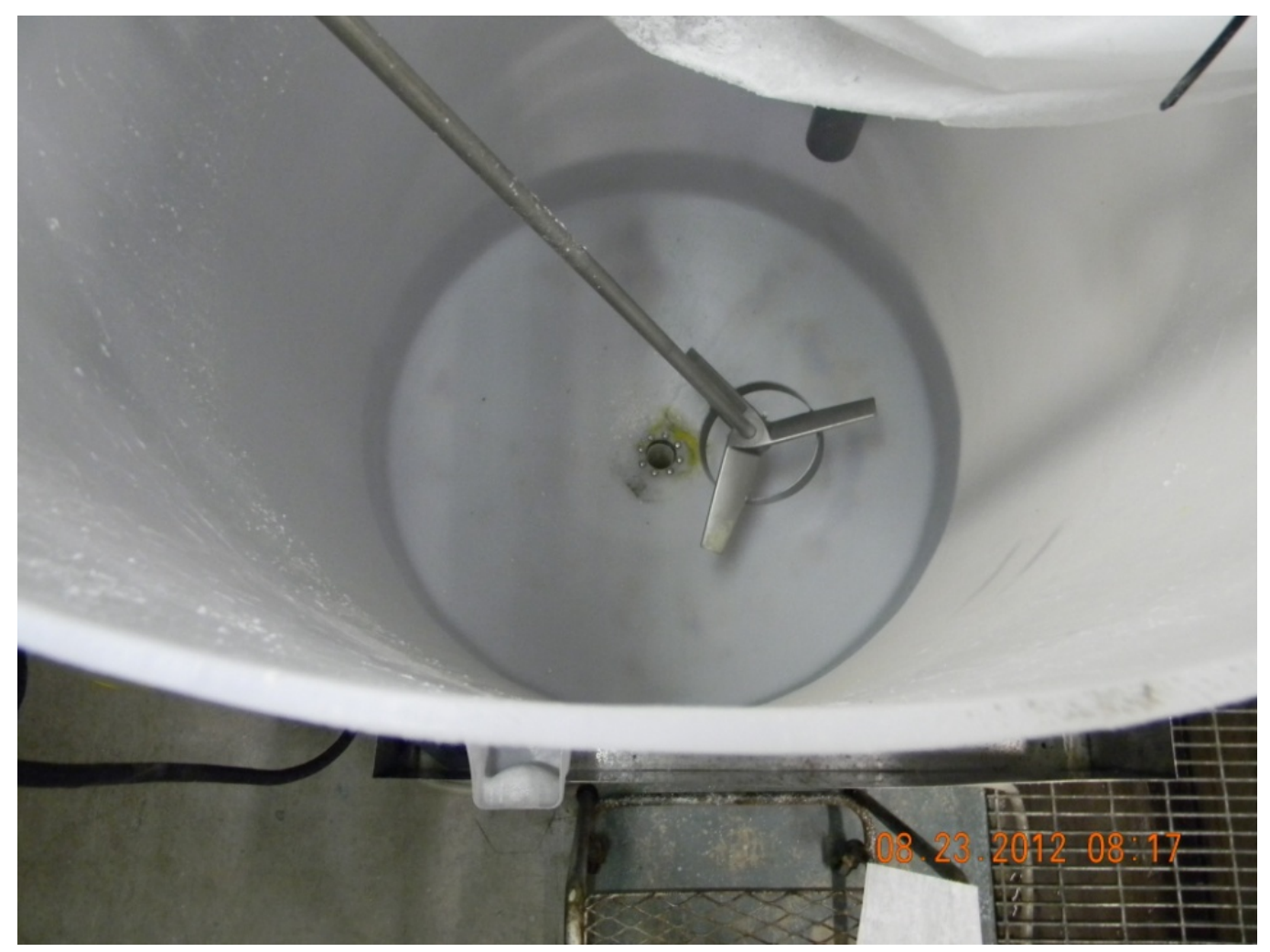

Figure 2-2. Conical-bottom feed tank showing agitator and drain port.

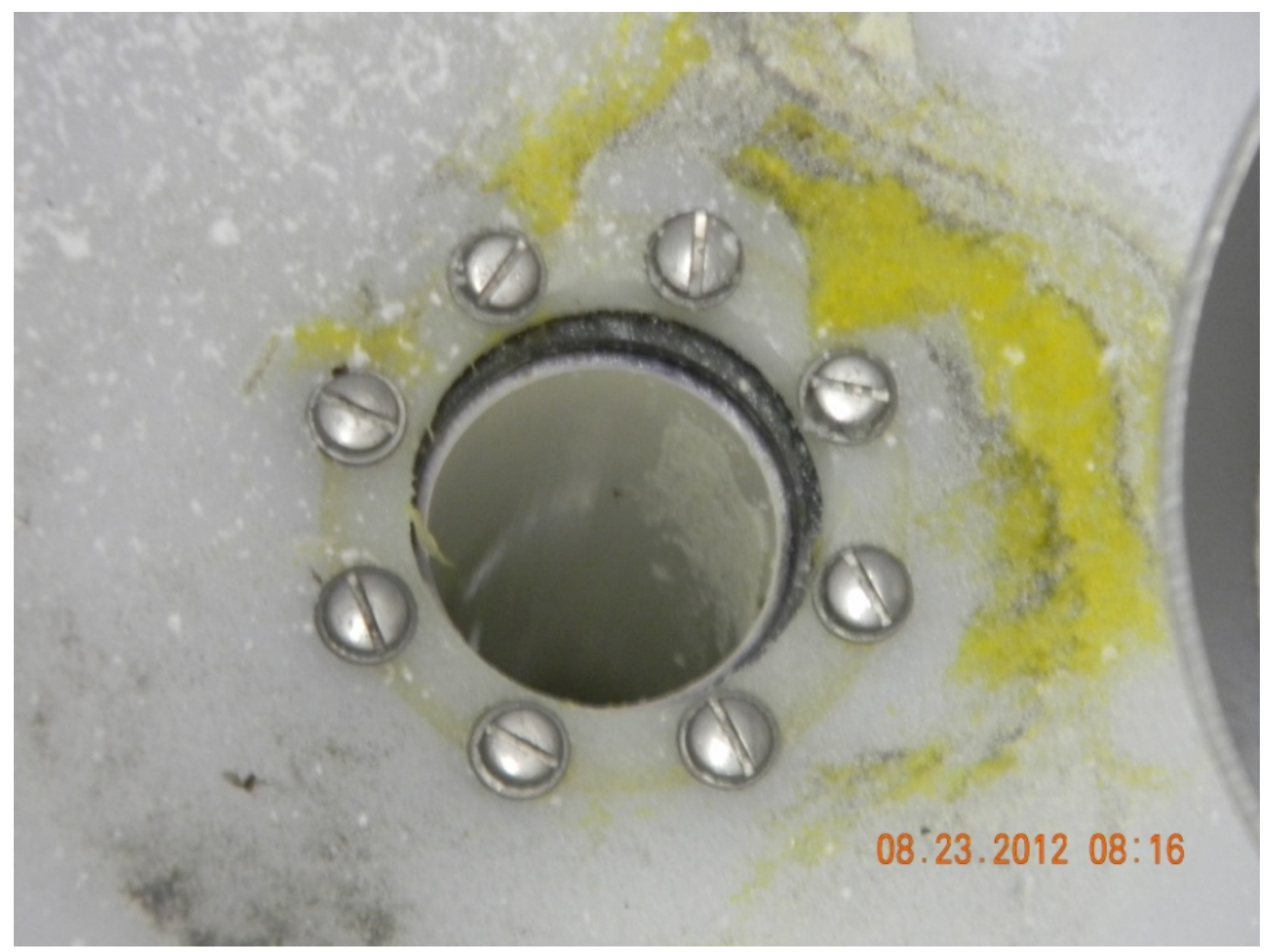

Figure 2-3. Close up of feed tank drain shown in the preceding figure. 
The most important issues while transferring feed to the MDT was to keep the solids suspended, keep them moving, minimize hold-up points, and include a least one flush with the volume to be transferred. The steps used to prepare the simulant and transfer it to the MDT were:

1. Feed is prepared and waiting in the feed tank - generally it was made ahead of time and sitting in the feed tanks with no agitation - All but the SS solids are in the tank and only about $80 \%$ of the supernatant.

2. Turn on the double diaphragm pump to begin circulation and re-suspend the sand, zirconia, and gibbsite.

3. Turn on the agitator to improve the mixing in the feed tank.

4. When the feed appears to be well mixed and well agitated slowly add the SS solids and wait 10-15 minutes to incorporate these solids.

5. Direct valve FT3 to the MDT, through the FLOW4 flowmeter, which was gravity fed.

6. When the simulant falls below the agitator it is shut off.

7. When most of the simulant is transferred and the pump begins to suck air through the system add enough supernatant directly into the top of the feed tank (separate line and pump) to flush the solids out of the bottom of the feed tank and feed system.

8. Repeat step 7 once to more thoroughly flush the feed system. (After a few times only one flush was used because it seemed to fully clean out the feed tank.)

9. Stop transfer when the MDT is full. At this point the MDT wa at the correct concentration of solids and there was generally some supernatant still in the feed system because more was put in than needed, which was used for the next batch.

\section{$2.2 \underline{\text { Simulant }}$}

In developing the simulant the starting point was to work with simulant material available from past SRNL testing [5-6] that included both a caustic supernatant and solids particles that would remain as undissolved solids (UDS) in the simulant, i.e., gibbsite and stainless steel particles. Based on published recommendations [8], and input from WRPS, the SASS project used stored supernatant that had a density of $1.28 \mathrm{~g} / \mathrm{mL}$ and viscosity of $2.7 \mathrm{cP}$, measured at $20^{\circ} \mathrm{C}$, and four solid particles of Gibbsite, Zirconium Oxide, Sand, and Stainless Steel. The last particle represented the large density solids in waste, e.g., plutonium, and was measured in the accumulated solids to determine its deposited distribution. Furthermore, the solids loading range of the wastes to be fed to tank AW-105 is estimated at 0.44 to $203 \mathrm{~g} / 1$, with an average of $89 \mathrm{~g} / \mathrm{l}$, but WRPS recommended using $100 \mathrm{~g} / 1$ for SASS and it was.

The supernatant was a Newtonian caustic solution with $\mathrm{pH} \sim 13$ made to represent Hanford waste. This liquid simulant is excess simulant from a previous test [11], but was deemed acceptable by WRPS to use for the present test. Because there were least eight 300 gallon totes of supernatant in storage at SRNL it was immediately available at no charge. Its chemical composition, Table 2-2, was developed to represent typical Hanford waste tank supernatants [11]. 
Table 2-2. Supernatant portion of SASS simulant used to suspend undissolved solids at $20^{\circ} \mathrm{C}$

\begin{tabular}{|c|c|c|}
\hline \multicolumn{2}{|c|}{$\begin{array}{c}\text { Sample } \\
\text { Species }\end{array}$} & \multicolumn{1}{c|}{$\begin{array}{c}\text { Unit } \\
\text { Date }\end{array}$} \\
\hline $\mathrm{Al}$ & $\mathrm{mg} / \mathrm{L}$ & 8945 \\
\hline $\mathrm{K}$ & $\mathrm{mg} / \mathrm{L}$ & 5915 \\
\hline $\mathrm{Na}$ & $\mathrm{mg} / \mathrm{L}$ & 137000 \\
\hline $\mathrm{P}$ & $\mathrm{mg} / \mathrm{L}$ & 1100 \\
\hline $\mathrm{S}$ & $\mathrm{mg} / \mathrm{L}$ & 4125 \\
\hline $\mathrm{F}$ & $\mathrm{mg} / \mathrm{L}$ & $<100$ \\
\hline $\mathrm{Cl}$ & $\mathrm{mg} / \mathrm{L}$ & 2960 \\
\hline $\mathrm{NO}_{2}$ & $\mathrm{mg} / \mathrm{L}$ & 25850 \\
\hline $\mathrm{NO}_{3}$ & $\mathrm{mg} / \mathrm{L}$ & 187000 \\
\hline $\mathrm{SO}_{4}$ & $\mathrm{mg} / \mathrm{L}$ & $<100$ \\
\hline $\mathrm{PO}_{4}$ & $\mathrm{mg} / \mathrm{L}$ & 2585 \\
\hline $\mathrm{C}_{2} \mathrm{O}_{4}$ & $\mathrm{mg} / \mathrm{L}$ & 11350 \\
\hline Density & $\mathrm{g} / \mathrm{mL}$ & 1.28 \\
\hline $\mathrm{pH}$ & - & 13.1 \\
\hline $\mathrm{Viscosity}$ & $\mathrm{cP}$ & 2.7 \\
\hline
\end{tabular}

As for the solids, WRPS and SRNL agreed on 2/29/2012 that the SASS simulant would have a UDS composition approximately to what is shown in Table 2-3.

Table 2-3. Typical Conceptual Simulant as Modified for SRNL Testing

\begin{tabular}{|c|c|c|c|}
\hline SASS Component & $\begin{array}{c}\text { Density } \\
(\mathrm{g} / \mathrm{ml})\end{array}$ & $\begin{array}{c}\text { Median particle size } \\
\text { by volume, } \mu \mathrm{m}\end{array}$ & $\begin{array}{c}\text { Mass percentage in } \\
\text { undissolved solids (2) }\end{array}$ \\
\hline gibbsite & 2.42 & 11 & 71 \\
\hline Safety yellow sand & $2.4(1)$ & 293 & 13 \\
\hline zirconium oxide & 5.7 & 15 & 15 \\
\hline stainless steel & 8 & 125 & 100 \\
\hline Total Mass \% & & & \\
\hline
\end{tabular}

(1) The sand itself had a density closer to $2.65 \mathrm{~g} / \mathrm{mL}$, but it is coated with a colored resin that is lighter and giving the small density of approximately $2.4 \mathrm{~g} / \mathrm{mL}$.

(2) The UDS loading was $100 \mathrm{~g} / \mathrm{l}$.

The particle size distributions (PSD) of the UDS can be found in Appendix A: Particle Size Distribution for Simulant Solids. These particles show an overall PSD similar to that of the Hanford Typical Conceptional Simulant [8], Figure 2-4. 


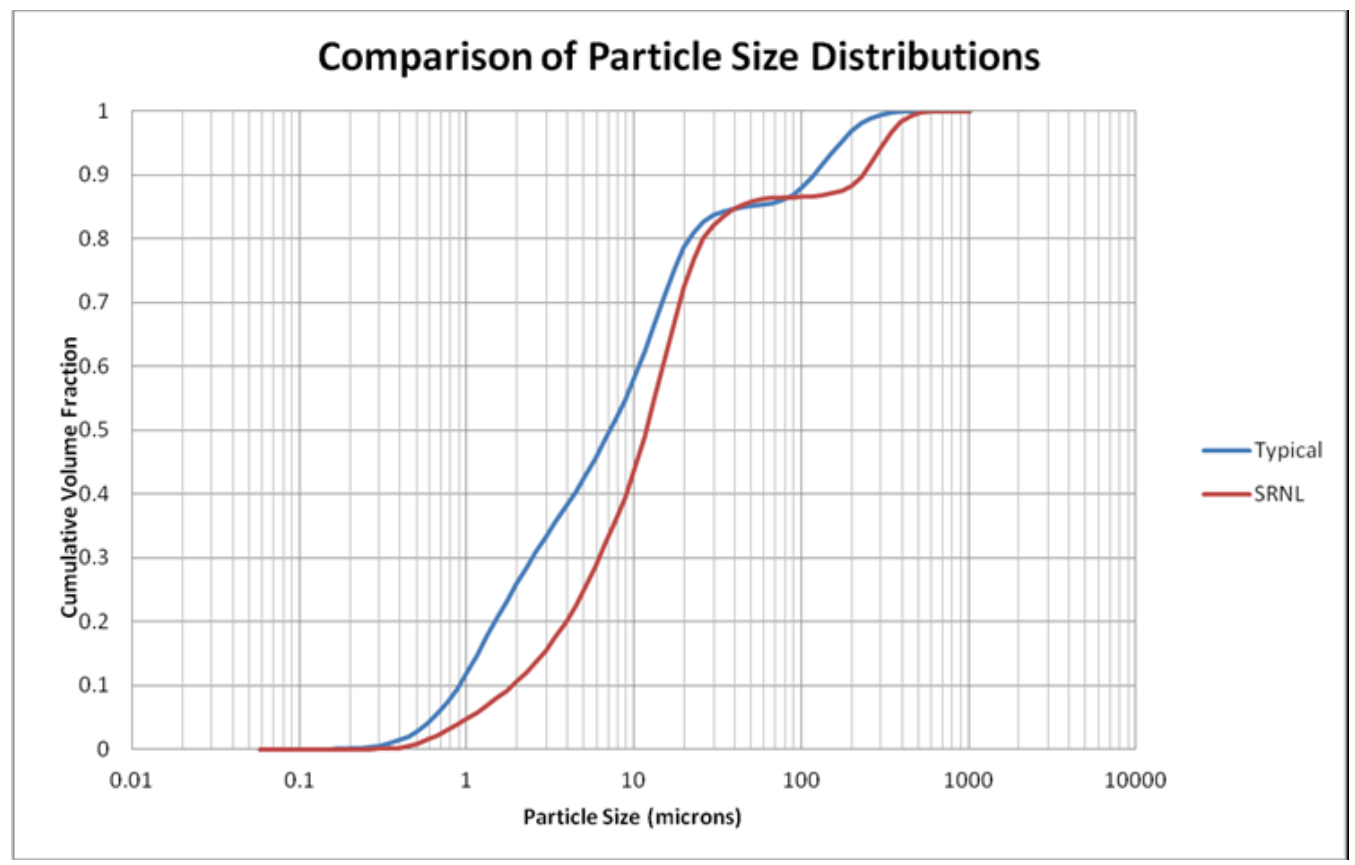

Figure 2-4. Particle sizes of SRNL simulant with four chosen UDS compared to the recommended [5] simulant.

When comparing the distribution of Archimedes number (Ar) of the SASS simulant to the Typical Conceptual Simulant [8] they are equivalent, Figure 2-5.

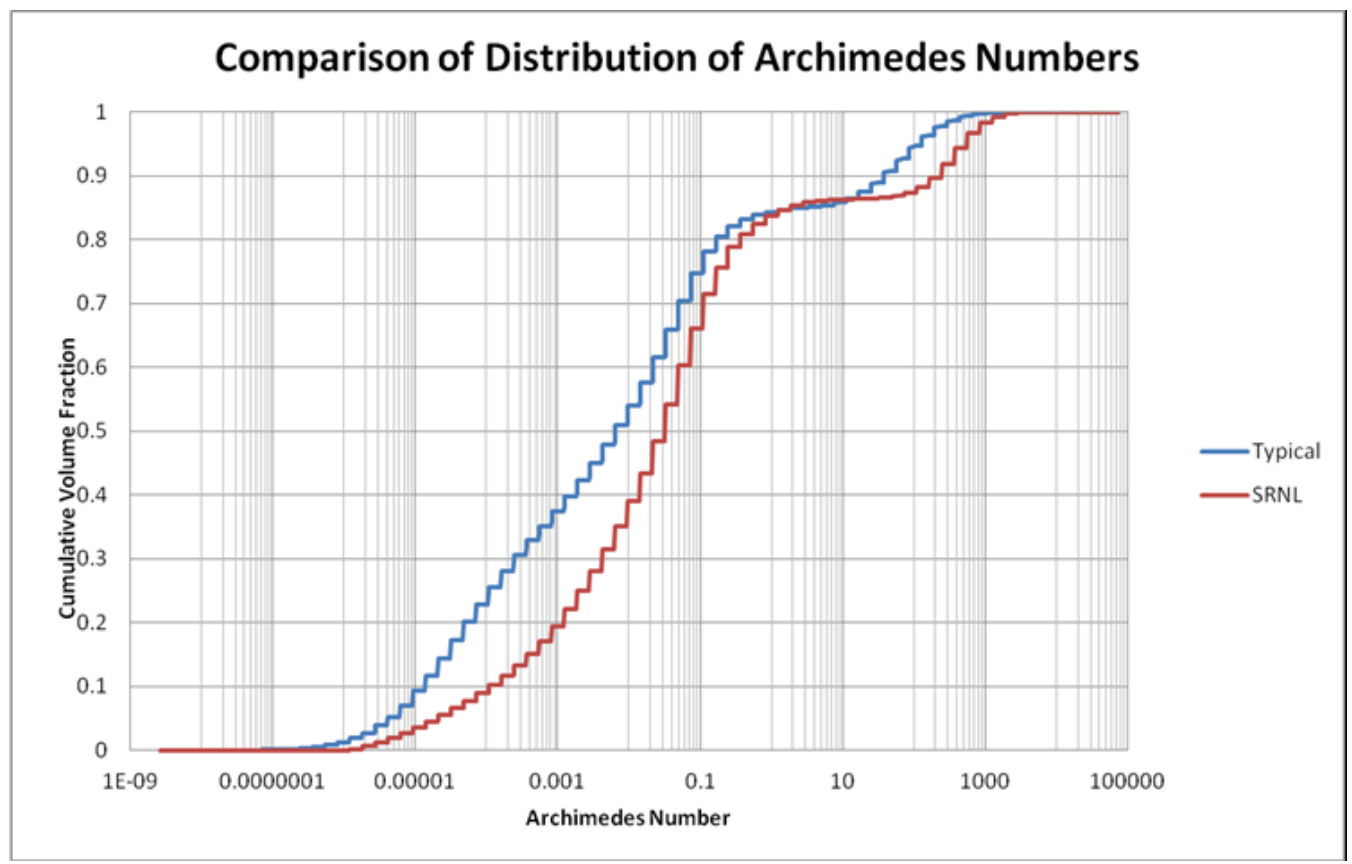

Figure 2-5. Settling characteristics of the SRNL simulant with four chosen UDS. compared to the recommended [8] simulant. 
Note that the Ar is a dimensionless number, Eq. (1), which is useful to compare the settling rates of different suspension slurries, i.e.,

$$
\operatorname{Ar}=\frac{\left(\frac{\rho_{\mathrm{S}}}{\rho_{\mathrm{L}}}-1\right) g \mathrm{gd}^{3}}{v^{2}}
$$

where $d$ is the particle diameter, $\rho_{\mathrm{S}}$ is the UDS density, $\rho_{\mathrm{L}}$ is the liquid density, $v$ is the kinematic viscosity of the liquid, which is the dynamic viscosity divided by the density of the liquid, and $\mathrm{g}$ is the gravitational constant.

\subsection{Utilization of the Measurement and Test Equipment}

The principal part of the SASS test facility was the Mixing Demonstration Tank (MDT) which is shown filled with simulant to the left side of Figure 1-1 and at the bottom of the piping and instrument diagram, Figure 2-1(a). However, the facility had many other parts that assisted testing in operation and measurement. Figure 2-1 shows the overall system and Table 2-4 lists some details of specific equipment and the calibrated uncertainties of the measurement devices. The following is an explanation of the system and its operation.

The small mixing system at the top of Figure 2-1(a) was to prepare and feed simulant. At the start of testing and at the end of each cycle a new batch of MDT feed simulant was made. Supernatant was pumped to the feed preparation tank, from the settling drums and through a 1micron filter, and put into circulation with Pump 4. Solids were weighed on the 0 to $45 \mathrm{~kg}$ scale and added to that tank. When ready, the contents of the feed tank was sent to the MDT through Flow Meter 4 until the MDT level reached 18.7 inches.

The MDT was then mixed by feeding the two jet pumps from Pump 1 and rotating, Figure 2-6, the two jet pumps in a clockwise direction and synchronized such that the jets from each pump were either pointing at each other or $180^{\circ}$ from each other, Figure 2-7. When ready a batch transfer was transferred from the MDT, through the magnetic separator, to one of the seven receipt tanks using Pump 3. After all the receipt tanks were full and level of sand was recorded in each then the contents of the receipt tanks were transferred to the settling drums to reuse the liquid portion of the simulant on the following day. During each cycle, and after each batch transfer, the stainless steel captured in the magnetic separator was collected. Later those samples were cleaned, dried, and weighed. 


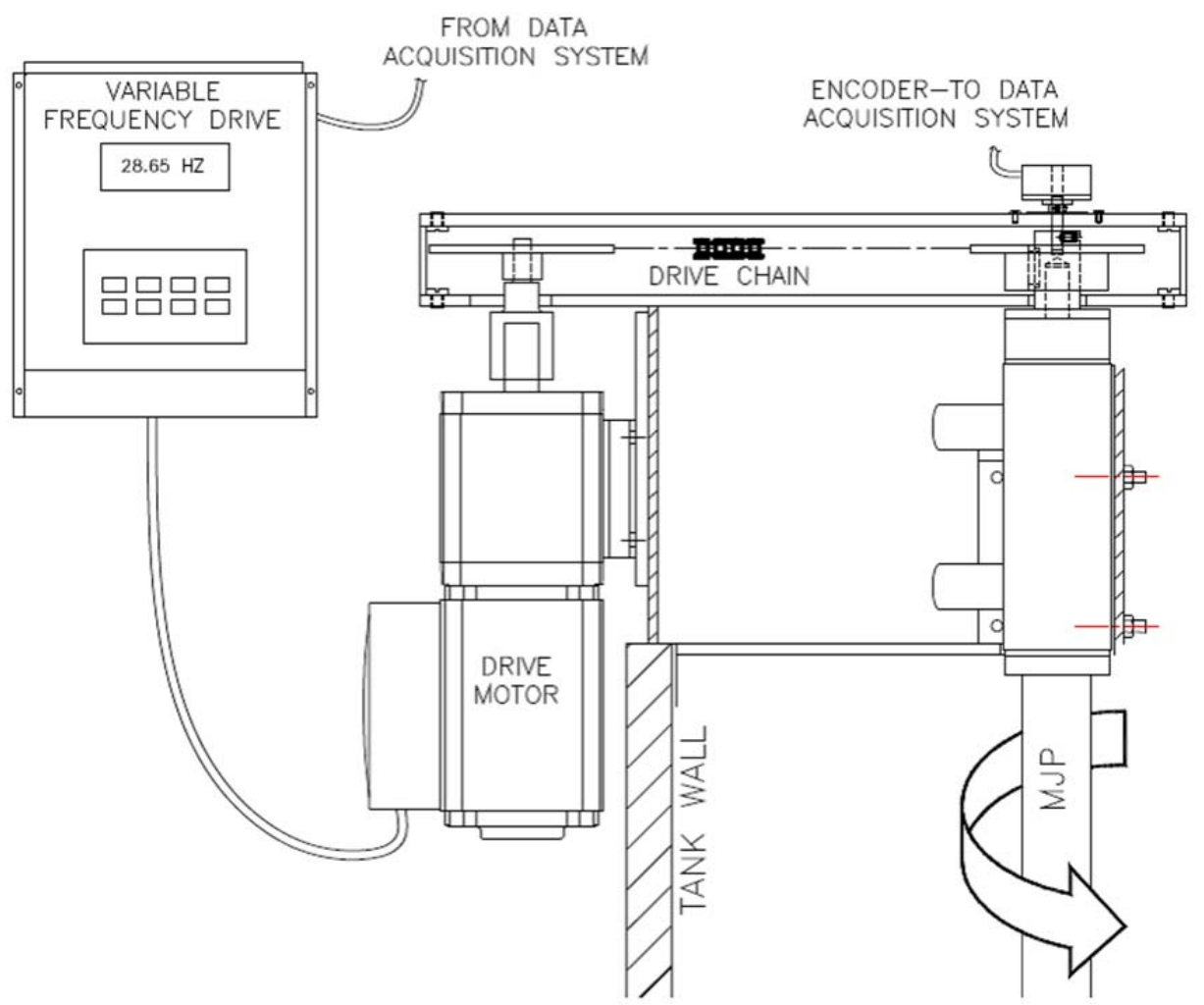

Figure 2-6. Illustrating one of the mixing jet pumps (MJP) and its system to rotate pump.

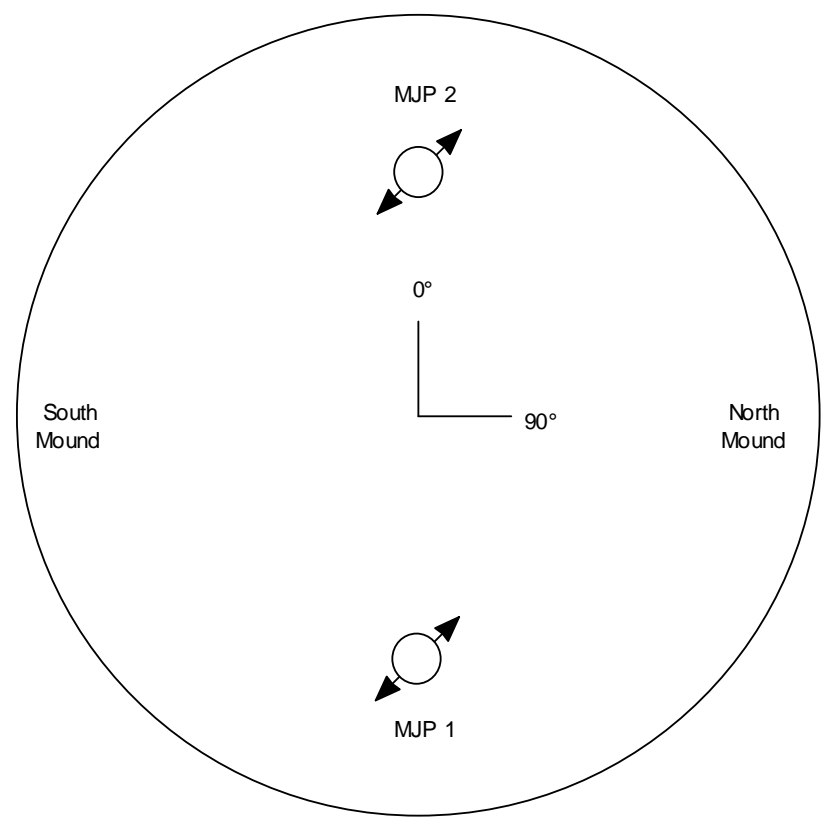

Figure 2-7. Top view of the MDT showing two MJPs and the angle convention. 
Table 2-4. Measurement and Test Equipment

\begin{tabular}{|c|c|c|c|c|c|c|}
\hline Location & Function & Instrument & Model & M\&TE\# & Calibrated Range & Accuracy \\
\hline \multicolumn{7}{|c|}{ Measurement and Testing Equipment } \\
\hline $\begin{array}{c}\text { Upstream } \\
\text { of MJP1 }\end{array}$ & $\begin{array}{l}\text { MDT Mixing } \\
\text { Flowrate }\end{array}$ & $\begin{array}{l}\text { FM1- Fischer-Porter } \\
\text { Magnetic Flowmeter }\end{array}$ & $10 \mathrm{D} 1475$ & TR-03674 & 0 to $25 \mathrm{gpm}$ & $\pm 0.4 \mathrm{gpm}$ \\
\hline $\begin{array}{c}\text { Upstream } \\
\text { of MJP2 }\end{array}$ & $\begin{array}{l}\text { MDT Mixing } \\
\text { Flowrate }\end{array}$ & $\begin{array}{l}\text { FM2- Fischer-Porter } \\
\text { Magnetic Flowmeter } \\
\end{array}$ & 10D1475 & TR-03811 & 0 to $25 \mathrm{gpm}$ & $\pm 0.4 \mathrm{gpm}$ \\
\hline $\begin{array}{c}\text { Upstream } \\
\text { of MJP3 }\end{array}$ & $\begin{array}{c}\text { Batch Transfer } \\
\text { Flowrate }\end{array}$ & $\begin{array}{l}\text { FM2- Fischer-Porter } \\
\text { Magnetic Flowmeter } \\
\end{array}$ & 10D1475 & TR-03680 & 0 to $1.6 \mathrm{gpm}$ & $\pm 0.04 \mathrm{gpm}$ \\
\hline $\begin{array}{l}\text { Upstream } \\
\text { of MJP4 }\end{array}$ & MDT Fill Flowrate & $\begin{array}{l}\text { FM2- Fischer-Porter } \\
\text { Magnetic Flowmeter }\end{array}$ & $10 \mathrm{D} 1475$ & TR-40134 & 0 to $4 \mathrm{gpm}$ & $\pm 0.03 \mathrm{gpm}$ \\
\hline In MDT & Temperature & $\begin{array}{c}\text { Barnant Thermister, } \\
\text { LogR }\end{array}$ & $600-1075 /$ YSI406 & TR-03667 & -40 to $125^{\circ} \mathrm{C}$ & $\pm 0.2^{\circ} \mathrm{C}$ \\
\hline $\begin{array}{l}\text { North Side } \\
\text { of MDT }\end{array}$ & Height & Leica Geosystems AC & 722508 Disto Pro4 & TR-40133 & 500 to $1000 \mathrm{~mm}$ & $\pm 1.5 \mathrm{~mm}$ \\
\hline $\begin{array}{c}\text { South Side } \\
\text { of MDT }\end{array}$ & Height & Leica Geosystems AC & 722508 Disto Pro4 & GT4-0003 & 500 to $1000 \mathrm{~mm}$ & $\pm 1.5 \mathrm{~mm}$ \\
\hline EDL & Mass Measure & Mettler-Toledo Scale & $8142 / 2045$ & TR-30074 & 0 to $45 \mathrm{~kg}$ & $\pm 0.045 \mathrm{~kg}$ \\
\hline EDL & Mass Measure & A\&D Balance & $\mathrm{HC}-3 \mathrm{KA}$ & TR-03525 & 0 to $3000 \mathrm{~g}$ & $\pm 2 \mathrm{~g}$ \\
\hline EDL & Mass Measure & $\begin{array}{c}\text { Denver Instruments } \\
\text { Balance }\end{array}$ & TR-403 & WP-951 & 0 to $400 \mathrm{~g}$ & $\pm 0.075 \mathrm{~g}$ \\
\hline \multicolumn{7}{|c|}{ Other Equipment } \\
\hline $\begin{array}{l}\text { Pump } 1 \\
\text { Upstream } \\
\text { MJP1\&2 } \\
\end{array}$ & $\begin{array}{c}\text { Circulates MDT } \\
\text { Simulant } \\
\end{array}$ & Galigher Pump & 1.5VRA1000 & N/A & $\begin{array}{l}\text { To } 180 \mathrm{gpm} \text { of } \\
\text { water at } 5 \mathrm{hp}\end{array}$ & N/A \\
\hline $\begin{array}{l}\text { Powers } \\
\text { Galigher }\end{array}$ & $\begin{array}{c}\text { Drives Galigher } \\
\text { Pump }\end{array}$ & Seimans Motor & LR 39020 & $\mathrm{~N} / \mathrm{A}$ & $\begin{array}{c}5 \mathrm{hp}, 230 \mathrm{VAC} \\
3 \mathrm{PH}, 60 \mathrm{~Hz}\end{array}$ & $\mathrm{~N} / \mathrm{A}$ \\
\hline $\begin{array}{c}\text { Pump } 2 \\
\text { Down- } \\
\text { stream RTs } \\
\end{array}$ & $\begin{array}{l}\text { Empties RTs to } \\
\text { Settling Drums }\end{array}$ & $\begin{array}{l}\text { Wilden Double } \\
\text { Diaphragm Pump }\end{array}$ & $\mathrm{T} 2$ & $\mathrm{~N} / \mathrm{A}$ & To $35 \mathrm{gpm}$ & N/A \\
\hline $\begin{array}{c}\text { Pump } 3 \\
\text { MDT to RT }\end{array}$ & Batch Transfer & Moyno Pump & 33201 & $\mathrm{~N} / \mathrm{A}$ & To $2 \mathrm{gpm}$ & $\mathrm{N} / \mathrm{A}$ \\
\hline $\begin{array}{l}\text { Pump } 4 \text { at } \\
\text { Feed Prep } \\
\text { Tank }\end{array}$ & \begin{tabular}{|c|} 
Send Supernate \\
to, and Mix \\
Simulant in, Feed \\
Prep Tank \\
\end{tabular} & Marlow ITT AC Pump & $11 / 2$ AOD-S & $\mathrm{N} / \mathrm{A}$ & To $35 \mathrm{gpm}$ & $\mathrm{N} / \mathrm{A}$ \\
\hline EDL & DAS+Controller & Dell Computer & Precision 690 & $\mathrm{~N} / \mathrm{A}$ & $\mathrm{N} / \mathrm{A}$ & N/A \\
\hline EDL & DAS+Controller & $\begin{array}{l}\text { NI Software: } \\
\text { Labview2009 }\end{array}$ & Version 9.0 (32 bit) & $\mathrm{N} / \mathrm{A}$ & $\mathrm{N} / \mathrm{A}$ & $\mathrm{N} / \mathrm{A}$ \\
\hline $\begin{array}{l}\text { On top of } \\
\text { MJP1\&2 }\end{array}$ & $\begin{array}{c}\text { Transmits } \\
\text { Rotation }\end{array}$ & $\begin{array}{l}\mathrm{NI} \text { - Encoders, } \\
\text { Incremental } \\
\text { Quadrature }\end{array}$ & $780251-1$ & $\mathrm{~N} / \mathrm{A}$ & $\begin{array}{l}0 \text { to } 16,000 \\
\text { revolutions }\end{array}$ & $\mathrm{N} / \mathrm{A}$ \\
\hline At each MJP & Rotates MJPs & $2 \times$ Croshopp motor & AC8040NV-PL73377 & $\mathrm{N} / \mathrm{A}$ & $\begin{array}{c}230 \mathrm{VAC}, 0.42 \\
\text { Amp, } 47 \mathrm{~W}, 3: 7.1 \\
\text { Ratio, } 3.7 \mathrm{rpm} \\
\end{array}$ & $\mathrm{N} / \mathrm{A}$ \\
\hline EDL & $\begin{array}{l}\text { Controls Motors: } \\
\text { Galigher and } \\
\text { Croshopp }\end{array}$ & $\begin{array}{l}3 \times \text { Leeson Variable } \\
\text { Frequency Drive }\end{array}$ & 174919 & N/A & $\begin{array}{c}0 \text { to } 60 \mathrm{~Hz}, 0 \text { to } 5 \\
\text { hp, } 208 \text { VAC }\end{array}$ & $\mathrm{N} / \mathrm{A}$ \\
\hline \multicolumn{2}{|c|}{ DAS = Data Acquistion System } & & \multicolumn{3}{|c|}{ M\&TE - Measurement and Testing Equipment } & \\
\hline \multirow{3}{*}{\multicolumn{3}{|c|}{$\begin{array}{l}\mathrm{FM}=\text { Flow Meter } \\
\mathrm{EDL}=\text { Engineering Development Laboratory } \\
\mathrm{MDT}=\text { Mixing Demonstration Tank }\end{array}$}} & \multicolumn{2}{|c|}{ MJP = Mixing Jet Pump } & & \\
\hline & & & \multicolumn{2}{|c|}{$\mathrm{NI}=$ National Instrument } & & \\
\hline & & & RT = Receipt Tank & & N/A $=$ Not Applicabl & \\
\hline
\end{tabular}


At the end of the $1^{\text {st }}, 5^{\text {th }}$, and $10^{\text {th }}$ cycles measurements of the volume and shape of the accumulated solids in the MDT were made by slowly draining the heel simulant that was being mixed to keep the small particles suspended. Measurement began by mapping the mounds with the two height-measuring lasers with the probe positioning system. The mapping was followed by taking core samples, using the same positioning system. Finally photographs were taken of the mounds as the heel was slowly reestablished in 0.2-inch increments. Details of these measurement techniques are included in the next section on pre-test trial runs.

\subsection{Pre-Test Trial Runs and Measurement Techniques}

Before the test began many aspects of the SASS test facility and measurement methods had to be evaluated. As described in the Test Plan [9] a series of trial test runs were performed; however, the order was changed as information from the trial test runs guided each subsequent step.

\subsection{Obtained Settled Sand Volumes}

A small sample of the full simulant was made and introduced into a small settling tube to observe the height of each of the settled solids. Another simulant was made up of just sand at the same concentration in the simulant supernatant and using the same diameter tube, 0.6 inch. The settled sand heights were compared and the two had the same heights indicating that the large sand particle $(293 \mu \mathrm{m})$ allowed the other solids to reside interstitially and not significantly affect the settled height. This was good information to show that the batch to batch transfers of the settled sand in the receipt tanks would be a good measure of the transferred sand material, Figure 3-1. 


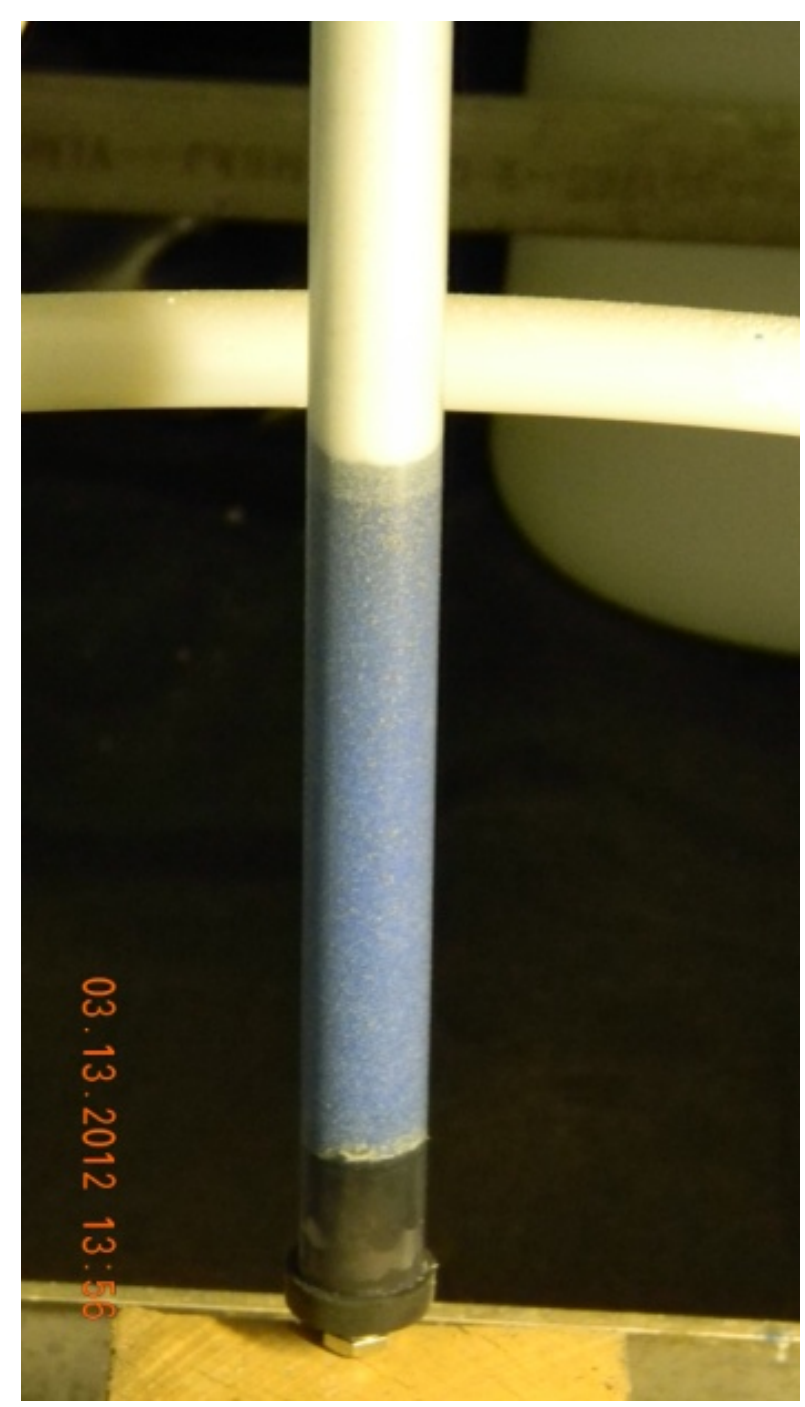

(a)

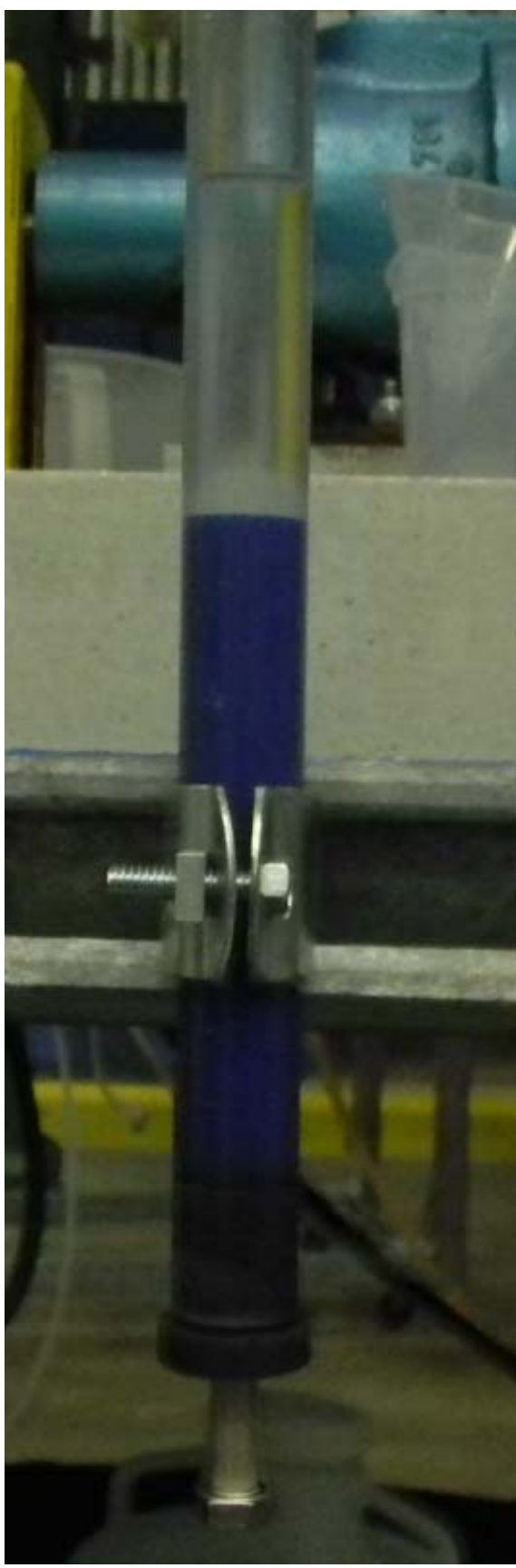

(b)

Figure 3-1. Sand settling test showing sand in the full simulant (a) had the same height as sand settling be itself (b) in only the simulant supernatant.

The tube that contained the full simulant also assisted in designing the receipt tanks by obtaining an approximate knowledge of the settling heights of the solids. The designed tanks are shown in Figure 3-2 with the full transfer batch volume of 13.13 gal (49.7 liters) and the partial batch volume of 6.88 gal (26.1 liters). 


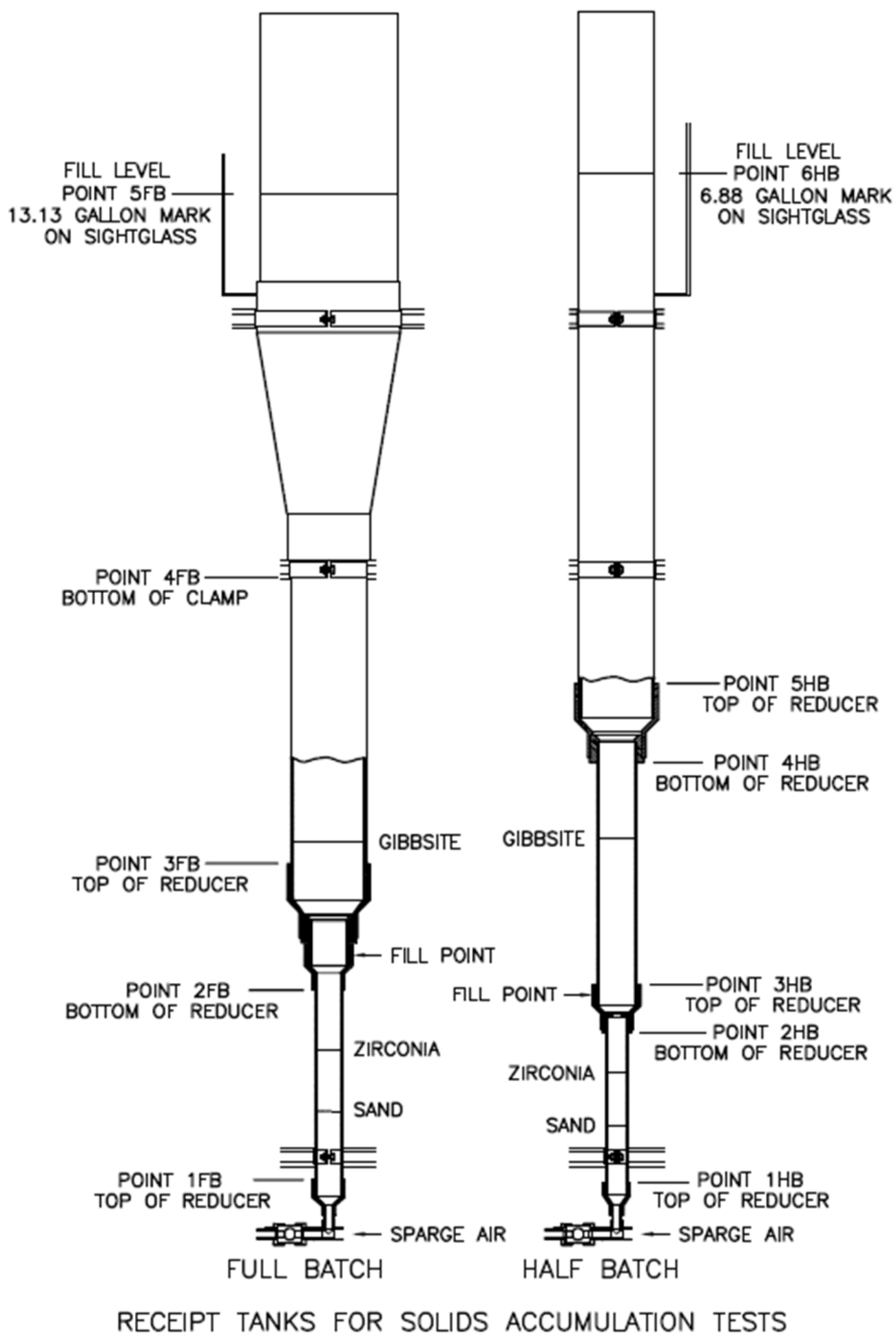

Figure 3-2. The design of the modified receipt tanks

to capture the sand, zirconia, and gibbsite particles from each transfer. However, only the sand would be consistently measured after 20 minutes of a transfer because the other particles were very small and took much longer to settle. 


\subsection{Determination of Removal Efficiency of Magnetic Separator}

A single cycle batch of 13.13 gal (49.7 liters) of simulant was made to test the magnetic separator, Figure 3-3. The batch was made in one of the Receipt Tanks, having the exact volume needed and transferred through the separator at 0.58 gpm into another Receipt Tank.

The amount of stainless steel collected was:

41.3 grams collected in the Magnetic Separator

13.5 grams were trapped in the Moyno Pump

54.8 grams total were recovered, which means that 5.1 grams were left in the Moyno pump even after being cleaned. However, before the test it was cleaned with just flushing with water, but after the test it was dismantled and cleaned by using a magnet to collect all the material. There was no stainless steel observed in the receipt tank after passing through the Magnetic separator; therefore, the separation was deemed successful.

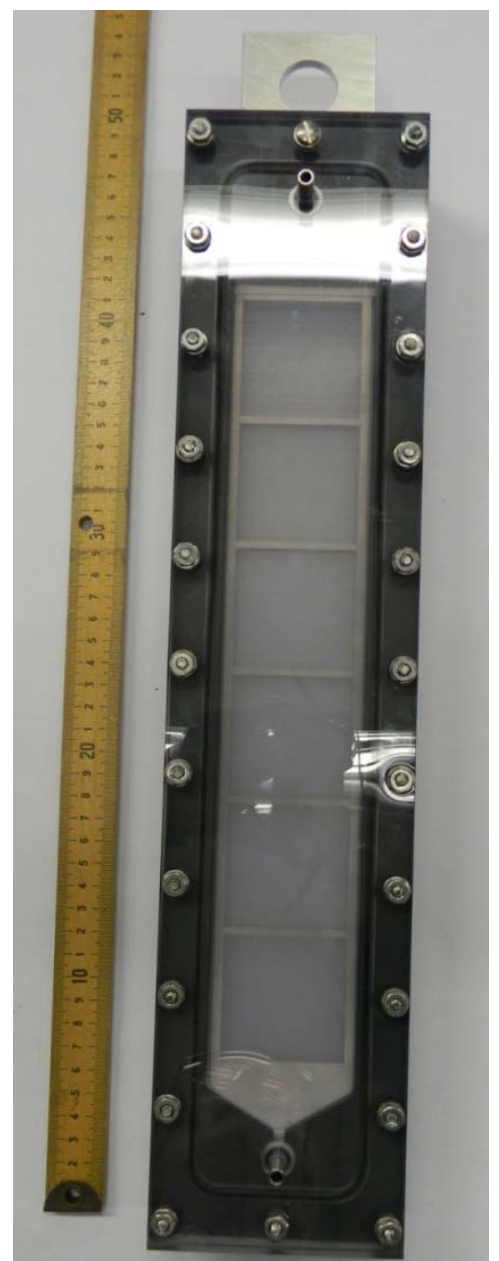

(a)

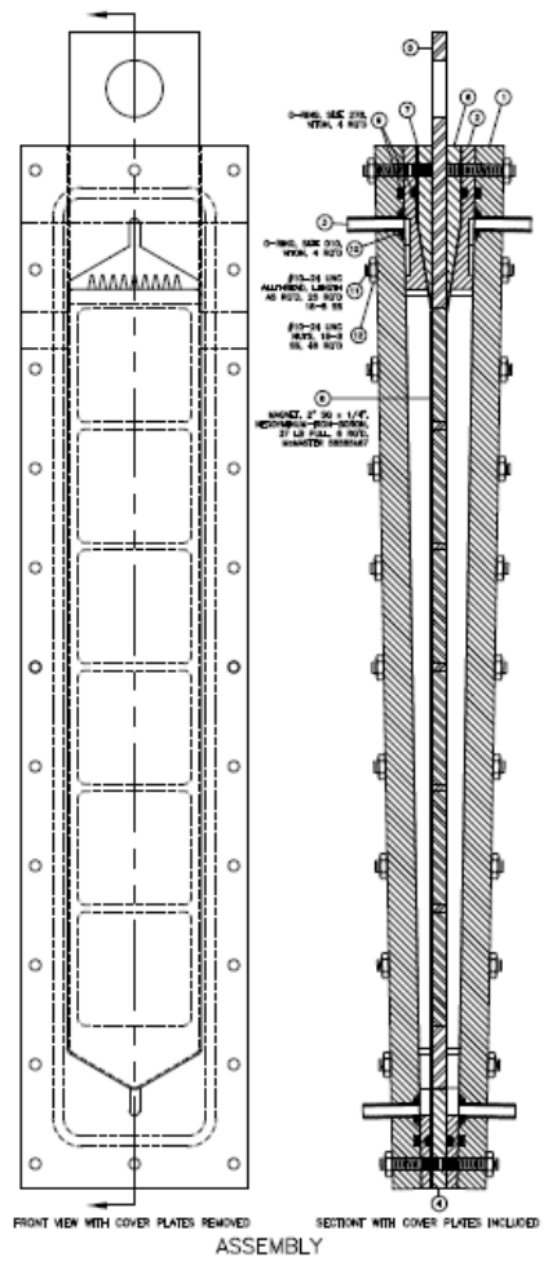

(b)

Figure 3-3. Magnetic Separator

(a) Photograph showing is 50-mm height, (b) As-built drawing. Not show is the protective aluminum face plate to better illustrate the device. 


\subsection{Determination of Jet Pump Flow Rate for Campaign 2}

This trial checked the operation of the receipt tank system and determined jet pump velocities that permit solids to accumulate on the bottom of the tank for Campaign 2. From past mixing MDT tests [5-7] at a given jet pump velocity if solids accumulate ${ }^{1}$ at all, they accumulate during initial tank mixing of 5 MDT volume turnovers and before a transfer began. Furthermore, there were clear solids accumulations at a jet pump velocity of $22.4 \mathrm{ft} / \mathrm{s} /$ nozzle (or $8 \mathrm{gpm} / \mathrm{pump}$ ) and a jet pump rotational speed of $1.57 \mathrm{rpm}$; therefore, this combination was used for Campaign 1 . However, accumulations at higher velocities/speeds have not been clearly defined. As such a series of jet pump velocities and jet pump rotational speeds were tested to determine the highest velocity/speed that allows solids to accumulate. The combinations of velocity/speed ${ }^{2}$ tried for Campaign 2 are listed in Table 3-1.

Table 3-1. Jet Pump Parameters used to determine Campaign 2 jet velocity

\begin{tabular}{|c|c|c|c|c|c|}
\hline $\begin{array}{c}\text { Nozzle } \\
\text { Velocity }\end{array}$ & $\begin{array}{c}\text { Nozzle } \\
\text { Flowrate }\end{array}$ & $\begin{array}{c}\text { Pump } \\
\text { Flowrate }\end{array}$ & $\begin{array}{c}\text { Pump } \\
\text { Rotation }\end{array}$ & $\begin{array}{c}\text { Scaling } \\
\text { Exponent }\end{array}$ & $\begin{array}{c}\text { Mixing } \\
\text { Time (1) }\end{array}$ \\
\hline $\mathrm{ft} / \mathrm{s}$ & $\mathrm{gpm}$ & $\mathrm{gpm}$ & $\mathrm{rpm}$ & - & $\mathrm{min}$. \\
\hline 32.3 & 5.76 & 11.5 & 2.37 & $1 / 5$ & 23 \\
\hline 27.7 & 4.94 & 9.9 & 2.03 & $1 / 4$ & 26 \\
\hline 24.4 & 4.35 & 8.7 & 1.79 & $7 / 24$ & 30 \\
\hline 22.4 & 4.00 & 8.0 & 1.57 & $1 / 3$ & 32 \\
\hline
\end{tabular}

(1) Mixing Time is the time necessary to attain turnover of the volume of simulant in the MDT five times. For a full tank was 103.6 gallons. The times listed were for the full-tank volume using the flowrates from both jet pumps.

To determine what the Campaign 2 jet pump velocity and rotation would be the operation performed was as follows:

1. A full batch, 103.6 gal (392 liters), of simulant was fed to the MDT at $1.75 \mathrm{gpm}$.

2. Once the MDT was full the jet pumps were started slowly and the pump head rotation synchronized.

3. The jet pumps were then set at the first flow parameters listed in Table 3-1.

4. After waiting for approximately 5 MDT turnovers no solids settling was observed and the pumps where stopped and a period of 2.5 hours were waited for settling to occur.

5. After the wait period the process was repeated with the next set of pump parameters.

6. Immediately after startup all of the settled solids were picked up and after 5 MDT turnovers, with no solids settling on the tank bottom, the next set of flow parameters were set without turning off the pumps.

\footnotetext{
${ }^{1}$ Actually the solids are more being picked up and redistributed in poorly mixed regions.

${ }^{2}$ As explained in Ref. 3, the scaling method uses exponents based the model criteria and for this task the chosen exponent, i.e., $1 / 3,1 / 4,1 / 5$, etc. is used to determine both the scaled jet pump velocity and jet pump rotational speed based on the full scale jet pump nozzle velocity of $60 \mathrm{ft} / \mathrm{s}$, jet pump rotation of $0.2 \mathrm{rpm}$, and tank diameter of 900 inches. That is, whatever exponent is chosen it determined both variables, which make them dependent. The scaling equations are:

$U_{j e t 2}=U_{j e t 1}\left(\frac{d_{\operatorname{tank2}}}{d_{\text {tank } 1}}\right)^{a}$ and $\omega_{\operatorname{tank2}}=\frac{\omega_{\operatorname{tank1} 1}}{S F^{1-a}}$ where SF $=1 / 22$ is the scale factor, subscript 1 is for full scale.
} 
7. With each successive drop in flow rate, i.e., 11.5, 9.9, and $8.7 \mathrm{gpm} / \mathrm{pump}$, an observation was made to look for settling solids, but the tank bottom remained solids free. Since the next set of flow parameters in Table 3-1 were to be used for Campaign 1, two more flowrates and corresponding rotation values were tested:

a. At $8.6 \mathrm{gpm}$ no solids settling was observed.

b. Finally at a jet flow rate of $8.4 \mathrm{gpm} /$ pump, i.e., a nozzle velocity of $\mathbf{2 3 . 5} \mathbf{~ f t / s}$ and a rotation of $1.72 \mathrm{rpm}$, solids began to settled on the tank bottom, or rather, could not be picked up; therefore, this flow setting was chosen for Campaign 2.

Note: If solids accumulation was to occur it happened almost immediately upon mixing the tank and the visible footprint of the solids mounds did not significantly change once the solids settled. Mixing for 5 MDT turnovers may not be necessary to obtain a good mix environment with respect to solids that settle.

\subsection{Overall Operation with Receipt Tanks, Magnetic Separator, and Solids Accumulation at Scaled Flow Rates}

After determining the Campaign 2 flow parameters from the previous trial test the full MDT was used to test the overall operation of the test facility. The MDT was mixed using Campaign 1 flow parameters, i.e., $8 \mathrm{gpm} /$ pump $(22.4 \mathrm{ft} / \mathrm{s})$ and a synchronized rotation of $1.57 \mathrm{rpm}$. After waiting for approximately 5 MDT turnovers the receipt tanks were filled one at a time at the planned rate of $0.58 \mathrm{gpm}$. During a transfer the magnetic separator was used to separate the stainless steel particles to determine the batch-to-batch operation of the separator. After filling of Receipt Tank 1 with the first transfer, the MDT mixing was stopped for 20 minutes and the magnetic separator of was emptied of SS solids and measured to check the separation process. A ruler was used to roughly measure the lengths and widths of the mounds of solids and the South mound was estimated at 20 long and 3 inches wide with the North mound being 18 inches long 4 inches wide, see Figure 3-4.

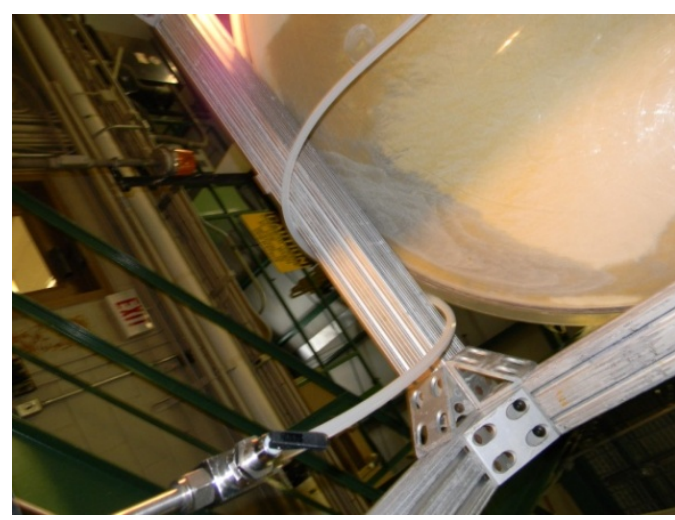

(a)

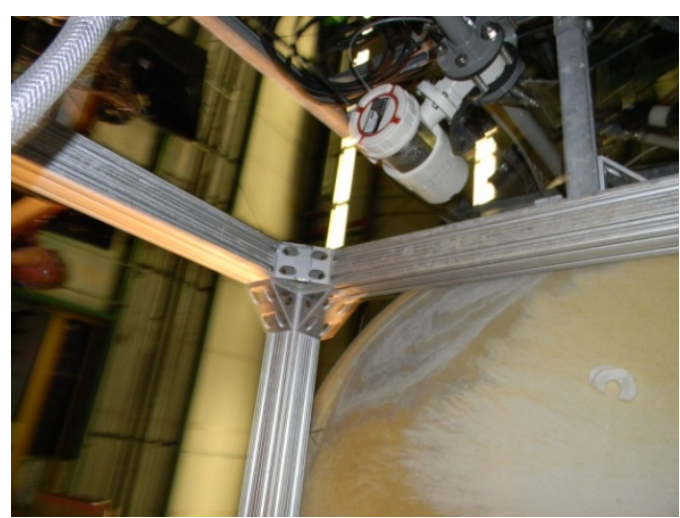

(b)

Figure 3-4. Pre-test solids accumulations during batch transfer to RT-1 (a) South mound, (b) North mound.

As soon as the magnetic separator was ready the entire transfer process was repeated once more filling Receipt Tank 2 and then the trial was ended for the day to allow the remaining tank volume to settle for the next trial run. 


\subsection{Wait Time between Each Feed Batch to Determine Effect on Accumulated Solids}

After waiting for 4 days (over the Memorial Day Holiday) the two filled receipt tanks were completely settled like Receipt Tank 1 shown in Figure 3-5.

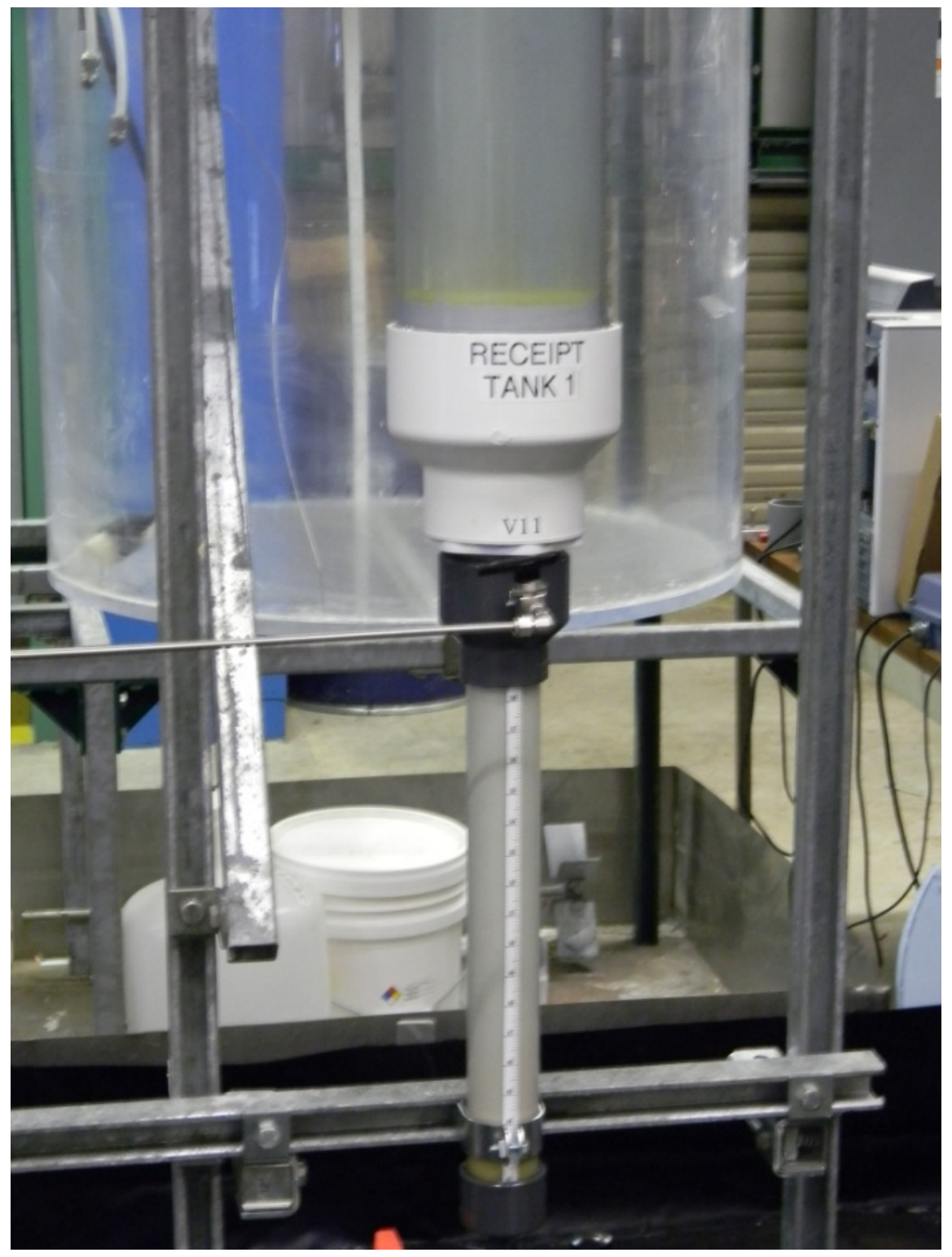

Figure 3-5. Solids settled in Receipt Tank 1 after four days.

From the previous trial the MDT still contained 5 more transfer batches, plus the heel volume. The remaining batch transfers were done and, on starting, the solids mounds were approximately the same size as they were before waiting 4 days. As the batch transfers progressed the mounds remained basically the same size or slightly smaller. It was concluded that either waiting 20 
minutes or 4 days between transfers did not seem to have a significant effect on the mound establishment; therefore, the 20-minute period was chosen to be used for the campaigns.

Along with evaluating the settling time, the settling in the receipt tanks was observed and the tanks securing brackets were readjusted to better observe the sand height (shown on the bottom of the RT1 in Figure 3-5). Once the MDT volume was reduced to the heel height, i.e., 3.23 inches, the jet pumps were stopped to begin the next trial test.

\subsection{Feed Rate to the MDT that Will Not Disturb Accumulated Solids}

After the preceding trial test the solids in the3.23-inch heel were allowed to settle for 75 minutes and then a refill stream of simulant was introduced at different flowrates to determine the maximum rate that would not disturbed the settled solids. The results obtained are in Table 3-2:

Table 3-2. Tank fill rate results

\begin{tabular}{|c|c|c|c|c|c|}
\hline \multicolumn{2}{|c|}{ Refilling MDT } & Height & Impingement & MDT & Mounds \\
\cline { 1 - 2 } Flowrate & Velocity & Above Heel & Point Disturbance & Diameter & Disturbed \\
\hline gpm & $\mathrm{ft} / \mathrm{s}$ & in & in & in & \\
\hline 0.6 & 1.7 & 16.3 & 2 & 40.4 & No \\
\hline 1.2 & 3.5 & 16.3 & 2.5 & 40.4 & No \\
\hline 1.5 & 4.4 & 16.3 & 2.8 & 40.4 & No \\
\hline 1.8 & 5.2 & 16.3 & 2.8 & 40.4 & No \\
\hline
\end{tabular}

The refill jet in the full scale tank was roughly estimated to impart 4 horsepower into the 72 -inch heel of waste, which is assumed to be too small to disturb accumulated solids in the dead zones near the tank wall. Therefore, any scale fill flowrate that did not visibly to disturb the solids was deemed acceptable; with the faster the better so the testing period can be kept at a minimum. From the results above the largest flowrate tested, i.e., $1.8 \mathrm{gpm}$, was used to refill the MDT.

3.7 Position Accuracy of the Mechanical Positioner to Measure Solids Mound Heights and Take Core Samples

A mechanical positioner, Figure 3-6 and Figure 3-7, was built that could easily be mounted and dismounted on top of the MDT. Computer control, Figure 3-8(a) rotated the positioner over a $110^{\circ}$ span and translated each sensor a distance up to 12 inches. The device had two sensor positions $180^{\circ}$ apart where laser rangefinders were mounted and two other positions, 3 inches from the rangefinders, where core sampling devices were mounted. 


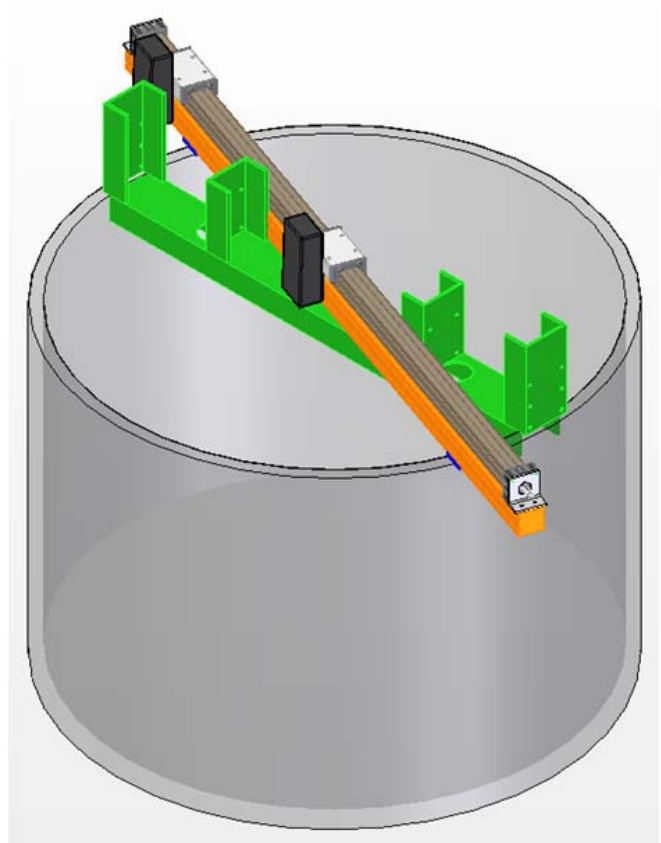

Figure 3-6. Mechanical positioner

The dark rectangles on the positioner arm indicate the laser rangefinders. In this initial sketch they are shown on the same side of the arm; however, during the trial run they were placed on opposite sides to increase the scan area.

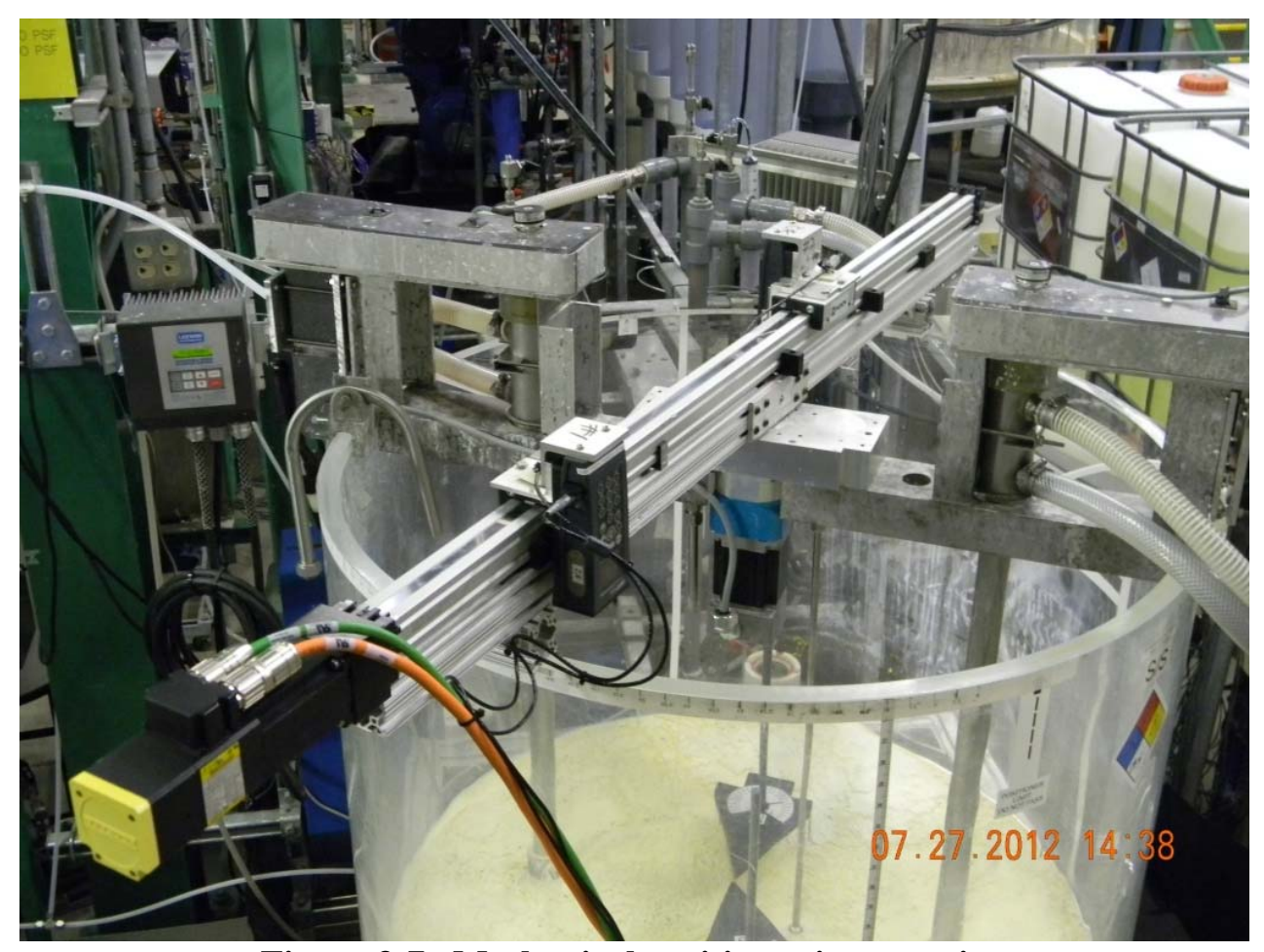

Figure 3-7. Mechanical positioner in operation

The laser rangefinders is mapping the bottom of the tank. 
Figure 3-8(b) shows a sensor position with the rangefinder-core-sampler combination on one side of the positioner arm. Actually the core sampler is not shown, but only the sampler holding bracket next to the rangefinder. The laser rangefinders move in tandem and as one rangefinder moved further from one side of the mixing tank wall the other rangefinder moved closer to the opposite wall. To measure solids mounds the positioner was set at $0^{\circ}$ and 0 -inch translation ${ }^{3}$. Height measurements were made by both rangefinders simultaneously and after each measurement the positioner was translated 0.75 inch. At the end of a series of height measurements, along a single angle, the positioner was returned to the 0 -inch translation location and then the positioner was rotated $2.5^{\circ}$. The process would then repeat until reaching the $110^{\circ}$ rotation position, resulting in a total of 1400 measurements.

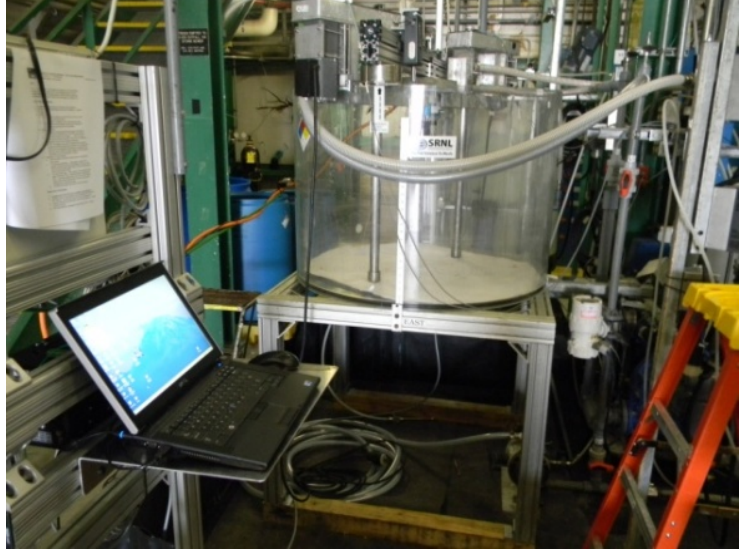

(a)

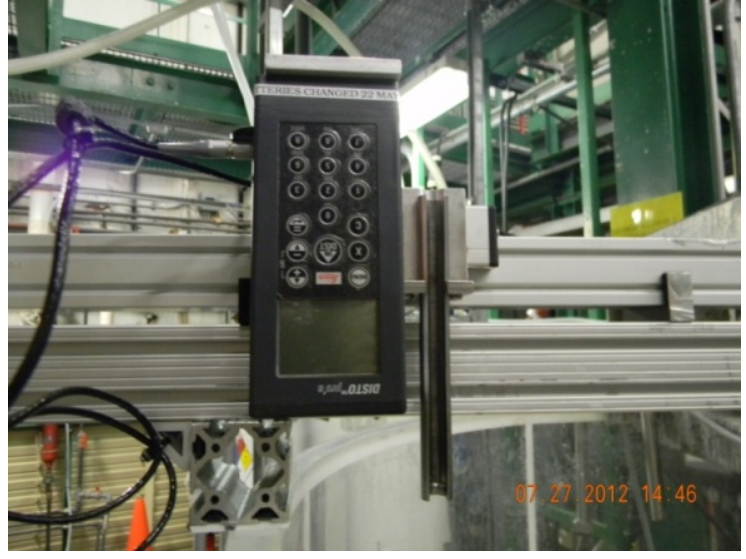

(b)

Figure 3-8. Calibration the laser system

(a) System was driven by a computer, (b) and made height measurements with a laser rangefinder - the core sampler fit in the slotted bracket shown next to the rangefinder.

The principle of operation for a laser rangefinder was to emit a short burst of light and measure the time for light to reflect from the object. Consideration was given to measuring through liquid in the tank but there were two problems. First, light travels slower in water than air by the factor of the refractive index. Second, the slowly settling suspended solids in the supernatant interfered with the laser beam, confusing the rangefinder.

Before installation on the positioner the two lasers to be used were calibrated for distance to \pm 0.04 inches, which is also the distance increment for the measurements. Once the lasers were installed the positions on the tank bottom were calibrated with a map of the laser measurement positions that was placed in the MDT, Figure 3-9(a). Many laser positions were checked and the positional accuracy was estimated at \pm 0.19 inches. Finally, the position accuracy of core sampler, shown in Figure 3-9(b) as the long skinny tube just above one of the red X marks, was also measured. Because of the long tube and being held at the top of the tank, the accuracy is estimated at \pm 0.38 inch. It was planned that the laser rangefinders be zeroed at tank bottom. However, this did not work because the tank bottom is transparent. Sometimes the rangefinder read the top surface of tank bottom, sometimes the bottom surface and sometimes the floor of the building.

\footnotetext{
${ }^{3}$ For the positioner its $0^{\circ}$ point was $47^{\circ}$ from the MJP centerline, see Figure 2-7, and the full $110^{\circ}$ rotation was from $47^{\circ}$ to $157^{\circ}$ to scan one mound and $227^{\circ}$ to $337^{\circ}$ to scan the opposite mound.
} 
After the calibration of the positioning system it was tested for its full operation of 1400 laser positions. Once the system was manually set to its zero position the computer took over. The system was preset to take a reading every $2.5^{\circ}$ and 0.75 inches and once started the entire process of mapping the tank bottom took an hour ${ }^{4}$.

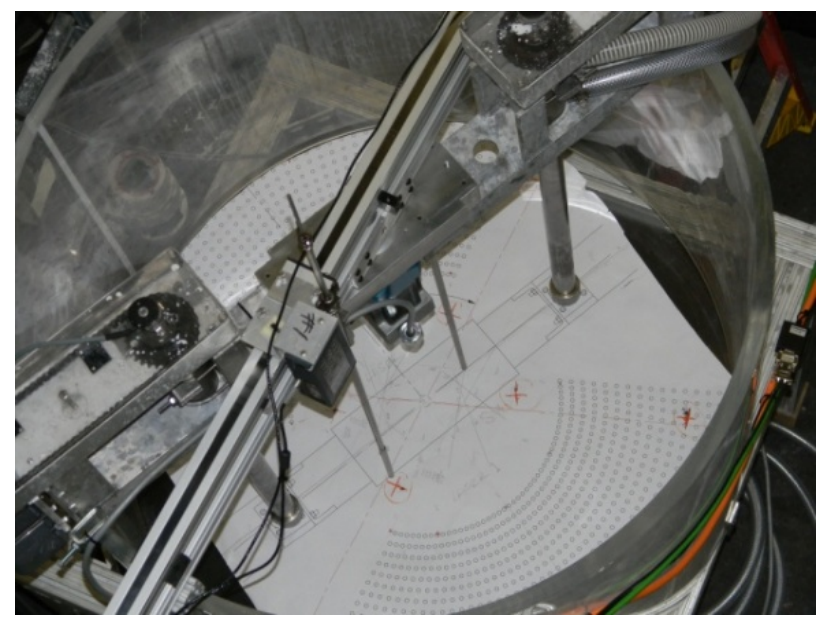

(a)

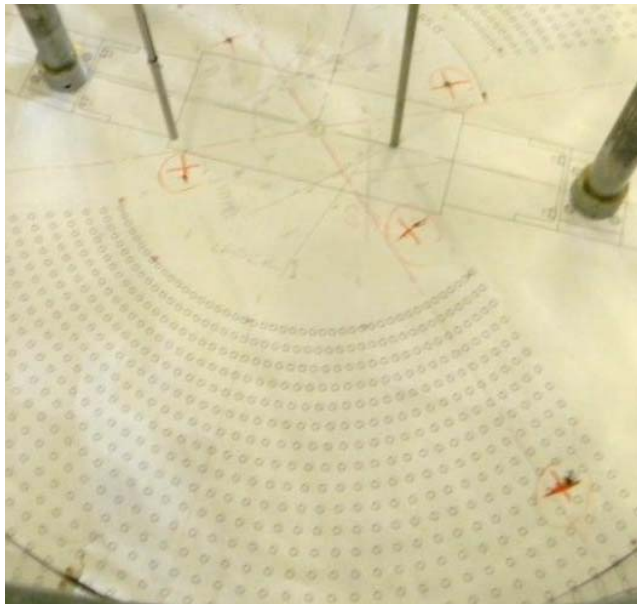

(b)

Figure 3-9. Positioner points being checked against full tank size grid map in relation to mixing jet pumps: (a) Positioner from above, (b) Grid Map.

\subsection{Measurement of Accumulated Solids Volume}

Two methods were used to measure volume: Laser rangefinder and 3-D topographical photographs. Because the heel supernatant affected the laser measurements is was necessary to first remove it. The original plan was to slowly drain the heel from the MDT, to not disturb the accumulated solids, at fixed intervals of height. At each interval the draining would be stopped, liquid heel height measured, and photographs taken. When the MDT was completely empty then a laser scan of the tank bottom would begin. Unfortunately, it turned out that the suspended, and settling, gibbsite and zirconia solids create problems in that a layer of these particles coated everything making the measurements difficult, especially the photographic technique because it was difficult to distinguish between the slurry and settled solids, which was necessary to determine volume. This solids coating also hampered the cores sampling somewhat because the fine gibbsite/zirconia layer on the top of the mounds would immediately backfill the holes where the core plugs were extracted. Therefore, the operation was changed to improve the measurement capability to the following:

\footnotetext{
${ }^{4}$ Note that during Campaign 1 the positioner rotation motor controller failed. Because of the time and cost to repair the controller was prohibitive the rotation was done manually. The tank was marked along its perimeter at the 45 radial measurement locations, i.e., $0^{\circ}$ to $110^{\circ}$. The computer still operated the linear movements of the lasers, along each radius, and recorded the data, but after each measurement sequence along a radius the positioner would be rotated manually to the next position, which didn't affect the time to make the laser scans and hopefully the accuracy of the radial location.
} 
1. Starting at the full heel height of 3.2 inches, mix the MDT as it would be at the end of the seventh transfer batch to suspend the fine solids. For example, for Campaign 1 the jet pump flowrate was $8 \mathrm{gpm} /$ pump and rotation was $1.57 \mathrm{rpm}$.

2. After at least ten minutes of mixing, stop the jet pump rotation at $0^{\circ}$ or $180^{\circ}$ so the jets are pointing away from the dead zones where the accumulated solids reside.

3. Reduce the jet pump flow to approximately $3 \mathrm{gpm} / \mathrm{pump}$ to have a flow that maintains the gibbsite/zirconia suspended but does not disturb the mounds. This was determined visually.

4. Start removing slurry at $0.8 \mathrm{gpm}$ through the central transfer tube while continuing to mix. Maintain this removal flowrate until the tank level is too low to allow it. During the process the mounds are closely observed for strong flow circulations that could disturb the settled solids. If it appears that a disturbance is possible the flowrate is dropped below $3 \mathrm{gpm} / \mathrm{pump}$. [Note, the actual changes in flowrates and levels as the heel was drained were chosen rather arbitrarily. The principal goal was to keep the gibbsite and zirconia suspended while not disturbing the accumulated mounds, primarily sand and stainless steel.]

5. Stop the jet pumps when the tank level reaches the bottom of the transfer pipe, i.e., 0.27 inches, and begins to draw air. At the same time open the MDT center drain to further drain the tank; however, once totally drained there was still approximately $1 / 8$-inch of material at the bottom that could not be removed by simply draining and therefore not removed.

6. Begin the laser scan of the bottom, which takes about one hour.

7. After completing the laser scan, use the positioner to place the core sampler to take samples at designated tank bottom locations. The size and shape of the mounds determined the locations.

8. After core sampling the heel was re-established by slowly ( 0.5 gpm was used) refilling the tank with clear supernatant. The rising level was stopped at measured increments and photographs were taken of the mounds.

9. Once the liquid level reached the top of the mounds the rest of the heel material, i. e, supernatant and solids, was returned to the MDT to reestablish the full 3.2 inches of heel.

\subsubsection{Volume by Laser Scan}

The process of obtaining volume from laser data began by adding three inches to the average distance measured to the tops of the two 3-inch tall black arrowhead-shaped indicator platforms, thereby obtaining an accurate distance measure from the laser to the tank bottom. (Note, these platforms, one of which is shown in Figure 3-10 and they are both shown in Figure 3-7, were placed in the MDT after the heel material was drained. They served to both verify laser heights, because they were exactly 3 -inch above the tank bottom had a scale that was manually set during the photographic technique so the liquid level was recorded. For example, Figure 3-10 shows the platform indicating 02 which meant a height of 0.2 inches of liquid. The letter $\mathrm{S}$ shown on the platform in Figure 3-10 indicated the South mound.) 
Each laser reading was subtracted from the laser-to-tank-bottom distance to give local mound height. Any measurement of mound height less than -0.02 " or greater than 20 " was assumed to be invalid and reset to zero. An Excel spreadsheet was used to plot the data for North and South mounds. Three dimensional plots were prepared in which the $\mathrm{x}$ axis was the angle of rotation with respect to the axis of the two pump heads, Figure 2-7, the y axis was the radial position, and the $\mathrm{z}$ axis was local mound height. To calculate mound volume, a trapezoidal area was associated with each laser measurement point. Areas further from the center of the tank had larger volumes than areas closer. Every height measurement was multiplied by its associated area to give an increment of volume. Increments of volume were added to give total mound volume, Eq. (2).

$$
V=\sum_{i=1}^{n} z_{i} A_{i}
$$

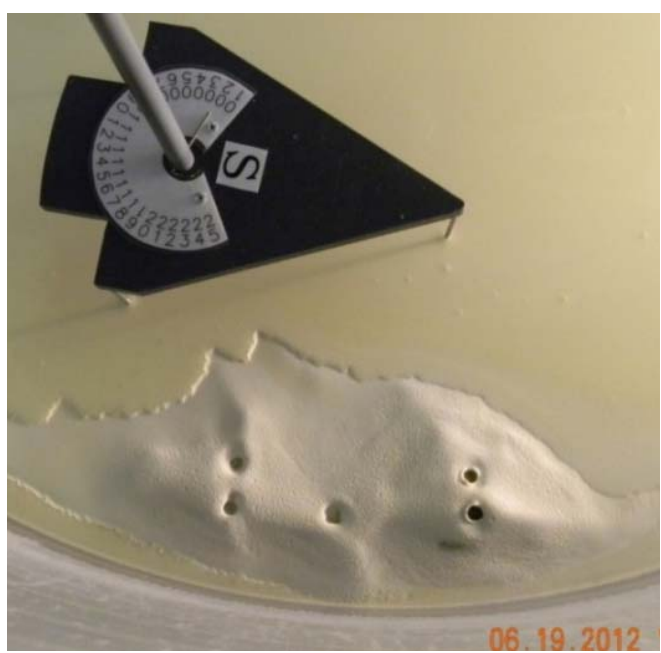

Figure 3-10. Triangular platform that stood 3 inches above the tank bottom. It also indicated which mound, e.g., $S=$ South, and the tank level by manually setting indicator, e.g., 0.02 inch.

To estimate the measurement uncertainty for the laser scan of the solids mounds both sand mounds and objects of known volume were placed in the tank, e.g., Figure 3-11. Table 3-3 shows an aluminum block was measured to $\pm 9 \%$ however, the block had straight sides. Because the laser positions were every 0.75 inch and $2.5^{\circ}$; therefore, this large uncertainty was not surprising. Furthermore, the measured volumes of the sand mounds depend on the packing density, so once again the range of measurement error obtained, i.e., \pm 1.9 to $\pm 8 \%$ was not surprising. The large plastic mound shown in Figure 3-11, gives a better representation of the measurement uncertainty because it was large (many laser points) and smooth (not large changes). However, the $\pm 0.3 \%$ obtained with the plastic mounds is believed much better than can be reasonably expected during actual used to measure real mounds because they were much bigger and wider than the actual mounds obtained. The measurement uncertainty was estimated at the average for the two sand mounds, or $\pm 5 \%$. The uncertainty may be better than the estimate, but more time would be necessary to determine this fact. 


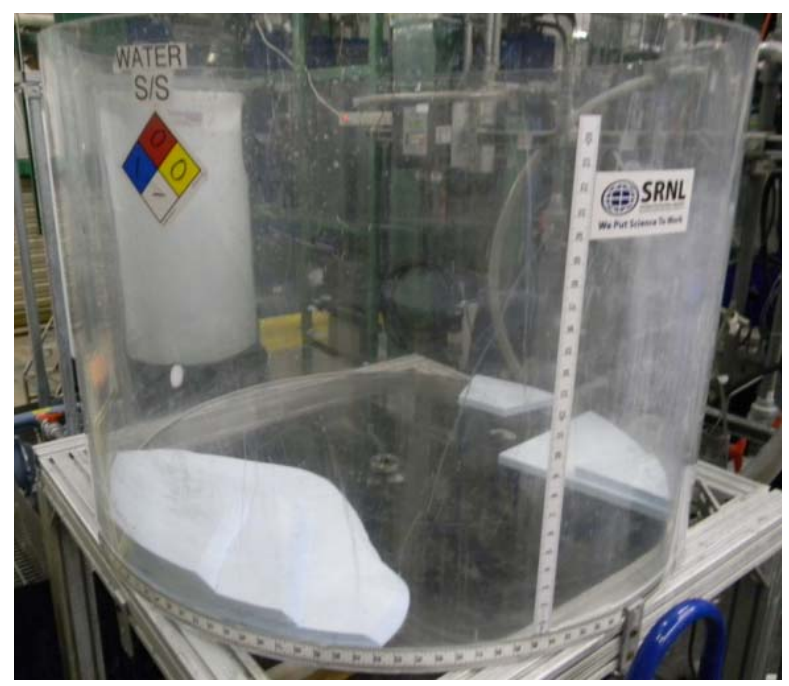

Figure 3-11. Determining volumes in the MDT with the laser using known volumes. Laser was not placed on the tank yet in the picture.

Table 3-3. Trial volumes for the laser to measure

\begin{tabular}{|c|c|c|c|c|}
\hline Date & Test Item of & $\begin{array}{c}\text { Known } \\
\text { Volumes }\end{array}$ & $\begin{array}{c}\text { Laser } \\
\text { Measure }\end{array}$ & $\begin{array}{c}\text { Difference } \\
\text { from Known }\end{array}$ \\
& Know Volume & inch $^{3}$ & inch $^{3}$ & Percent \\
\hline $5 / 15 / 2012$ & Sand Mound & 374 & 367 & $1.9 \%$ \\
\hline $6 / 21 / 2012$ & Aluminum Block & 65.1 & 59.4 & $8.8 \%$ \\
\hline $7 / 11 / 2012$ & Plastic Mound & 357 & 358 & $0.3 \%$ \\
\hline $7 / 11 / 2012$ & Sand Mound & 122 & 112.2 & $8.0 \%$ \\
\hline
\end{tabular}

\subsubsection{Volume by Photographs}

Each photographic image included one of the black plastic indicator platforms labeled $\mathrm{N}$ or $\mathrm{S}$ to designate which mound, Figure 3-10. While the series of photographs was taken of each mound the platforms were not moved to allow later alignment of those images. A pointer on each platform showed the tank level for the photograph and was manually increased to correspond to the increasing tank level. Furthermore, as the tank level increased the mounds formed islands of decreasing area. From the photographs each image was traced to follow shoreline of the islands after which the photographs were resized so that image of the black platform was the same length in each. The area inside the shoreline was determined for each photograph and scaled by the known dimensions of the black plastic platform. The result for each mound was a list of areas and associated elevations (tank heights). The first method used to integrate the data to yield volume was the Simpson's Rule [13, p. 171], Eq. (3).

$$
V=\frac{\Delta z}{3} \sum A_{1}+4 A_{2}+2 A_{3}+4 A_{4}+2 A_{5} \ldots+A_{n}
$$

Simpson's Rule worked well in a test using sand and water when performing the trial test for the photographic/volume technique with a mock mound shown in Figure 3-12. 


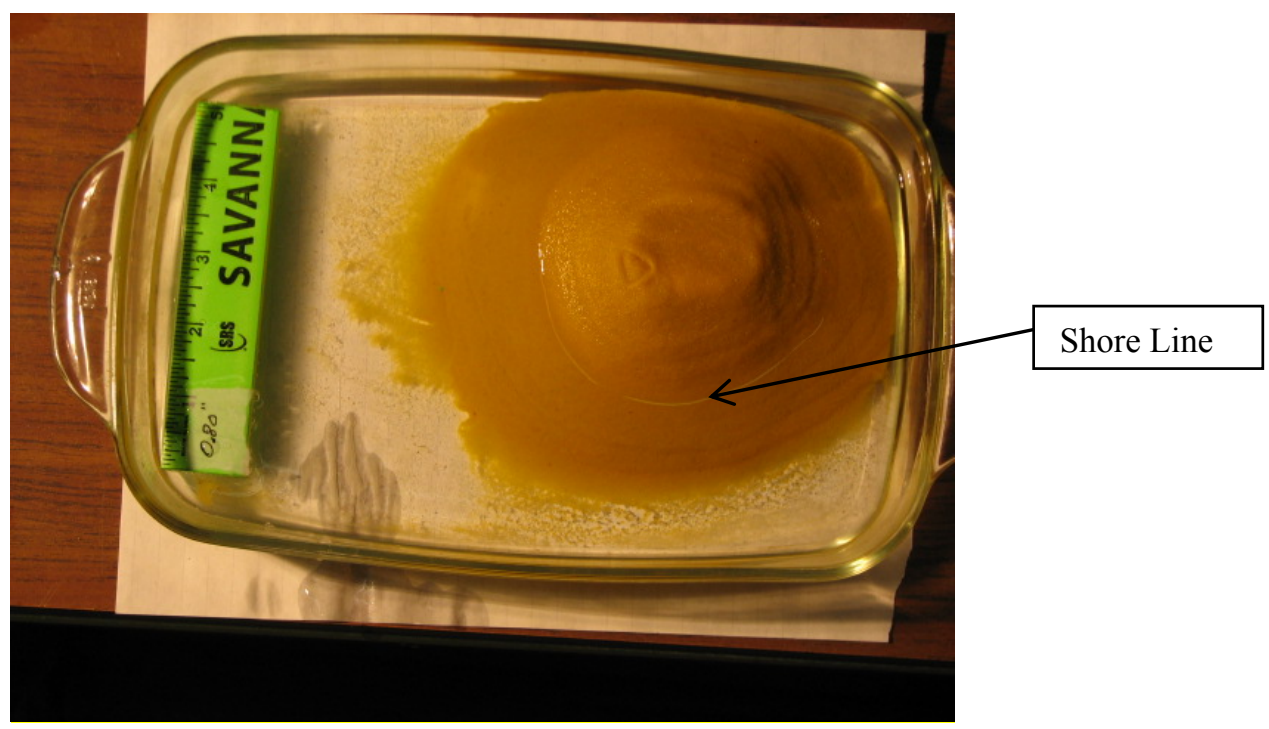

Figure 3-12. Yellow sand mound in water to determine contrast between liquid and solids, which is can be seen in this photograph, and estimate measurement uncertainty.

Photographs were taken at nine fixed liquid levels, e.g., Figure 3-12 is at 0.8 inches. For each photograph the shoreline between the island and the water was visually determined and manually superimposed on a drawing, Figure 3-13. The horizontal area of each elevation was measured and volume was computed as the integral of area with respect to elevation. In using the Simpson's rule the resulting volume was $20.1 \mathrm{in}^{3}$, which is within $3 \%$ of the volume of sand originally added. Unfortunately, the care and time necessary to perform this measurement by hand was prohibitive. A more practical method was to use digital images of the photographs, Figure 3-14, and from this process the volume was estimated at $21.6 \mathrm{in}^{3}$, or within $11 \%$ of the known volume. However, this is an ideal case, under controlled conditions, and the measurement uncertainty for the actual mounds will be higher.

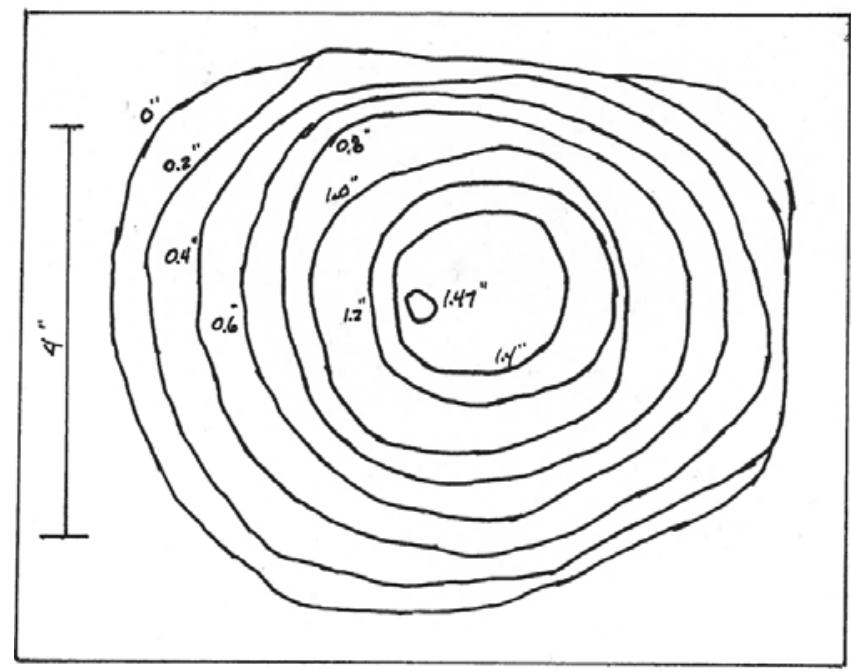

Figure 3-13. Contour plot of the solids mound shown in the preceding figure. 
However, a problem arose when using the photographic method in a MDT because of light colored and small sized solids. The residual layer of gibbsite and zirconia was difficult to make an area measurement for tank level less than 0.4 inch. If the increment of tank level was maintained at 0.4 inch, there would be too few measurements because mound height was usually less than 1.0 inch. Therefore, the Trapezoidal Rule [13, p. 171], Eq. (4) was used. This rule is less accurate but does not require that increments of tank level to be all equal.

$$
V=\sum_{i=1}^{n} A_{1}\left(z_{1}\right)+A_{2}\left(z_{2}-z_{1}\right)+A_{3}\left(z_{3}-z_{2}\right)+\cdots
$$

Based on this analysis the photographic technique the measurement uncertainty was estimated to be $\pm 20 \%$. However, for the actual test the volume of accumulated solids, as measured using the photographic method, was expected to have more uncertainty than the laser method because the:

a. Gibbsite/Zirconia that coated the mounds, and also in the liquid layer, made determining the "shore line" of the liquid/solids interface difficult.

b. The size of the mounds was small and shallow, i.e., less than 1 inch in height; therefore, there were fewer surfaces to estimate the volume. With fewer surfaces the Simpson's rule of integration would have to be replaced with the less accurate Trapezoid rule.

Because of these challenges, the measurement uncertainty was expected to be close to $\pm 20 \%$ for the larger North Mound, but higher for the smaller South Mound.
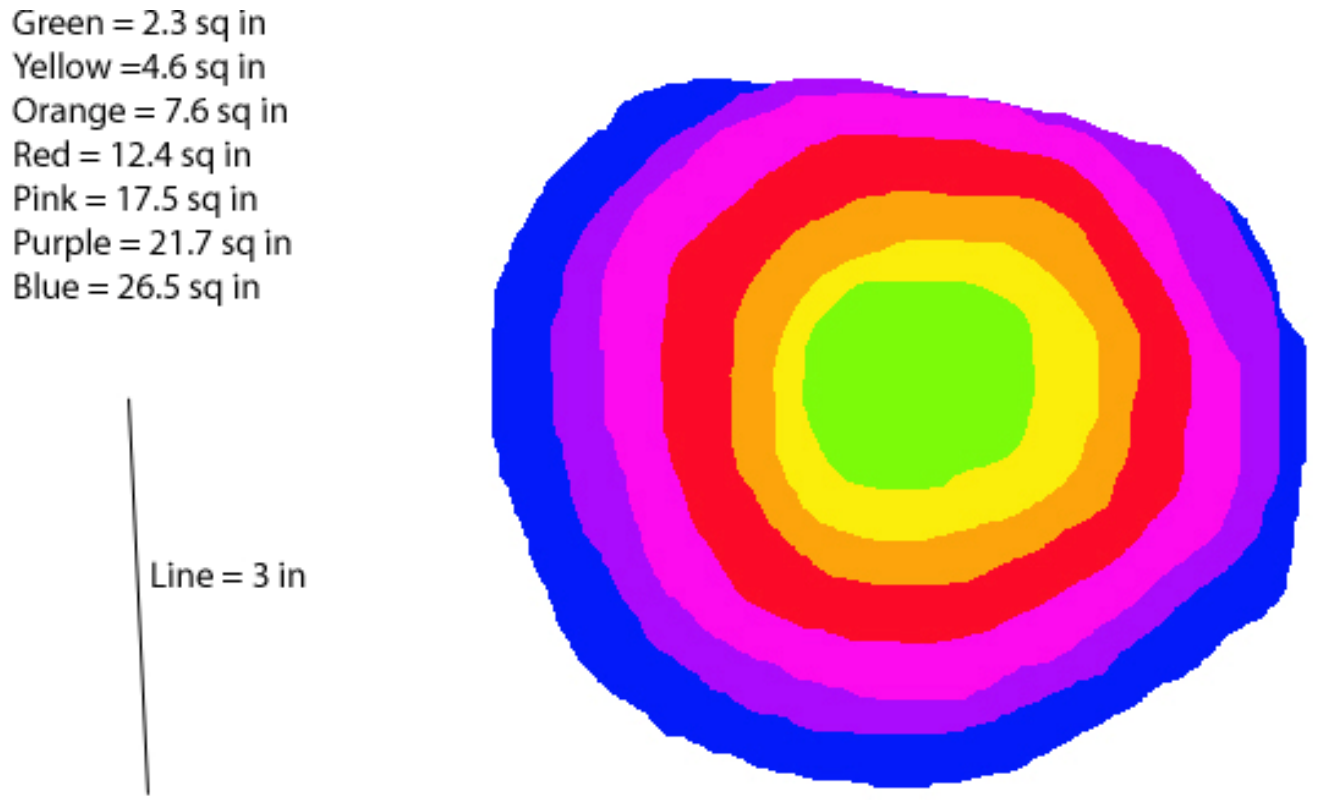

Figure 3-14. Digital contour plot of the solids mound shown in the preceding figure. 


\subsection{Core Sampler and Its Ability to Capture Complete cores}

Conventional sampling techniques with commercial equipment should suffice for most sampling needs. However, one critical need was for a device that could be placed at a precise location over the heel mound and collect a core sample with minimal disturbance to the mound.

The accumulated solids core samples were only taken after a full tank transfer, which means the slurry level was at the predetermined heel height of 3.2 inches, i.e., a scaled equivalent to the planned 72-inch heel of full scale operation. As previously explained, the heel liquid was slowly drained from the tank to measure the volume of the accumulated solids. Once the supernatant and suspended solids were fully drained to expose the mounds of accumulated solids the core sampler was placed in the positioner bracket, next to one of the laser, Figure 3-8(b), and then moved to a chosen location to obtain a core sample.

The concept that was developed uses the a "finger over the straw" technique, that is, pushing a thin-wall tube into the heel mound, and then sealing off the top of the tube to lift the captured core sample out of the mound. Of course, implementing this simple process without disturbing the mound significantly and doing it at the desired location requires a more sophisticated technique. Figure 3-15 shows the device constructed and the numbers in the following text correspond to the numbers shown in Figure 3-15(a).

The core sample incorporates a thin wall outer tube, 1, with an O-ring sealed plug, 9, where the tube and seal can move relative to each other. The general operation involves the following steps:

1. Using the wing nuts to unlock the outer tube, 1 , and plug supporting tube, 3 , adjust the outer tube, 1, and plug, 2, so the plug is flush with the bottom of the tube and lock in place.

2. Secure the sampler support, 4, in the positioner system bracket, Figure 3-8(b), and move the positioner arm to locate the sampler a desired place on one of the solids mound.

3. Lower the sampler until the bottom of the tube, 1, and plug, 2, are just touching the top of the mound and lock at that elevation.

4. The sampler tube is pushed, by unlocking the top wing nut and using the out tube positioning rod, 6, vertically downwards, past the stationary seal plug, 2, into the mound until it reaches the tank bottom and then lock the top wing nut to maintain the relative positions of the tube to the plug.

5. Lift the entire sampler unit with the captured sample of solids out of the support bracket, Figure 3-8(b).

6. Discharged the captured core from the sampler by unlocking plug and move the tube relative to the plug to push out the core over a suitable catch dish or sample paper. Cores of sufficient length are then separated into pieces and stored separately so that the concentration of stainless steel in each piece can be measured. [In the Results Section show two examples are shown: Figure 4-61 and Figure 4-62] 


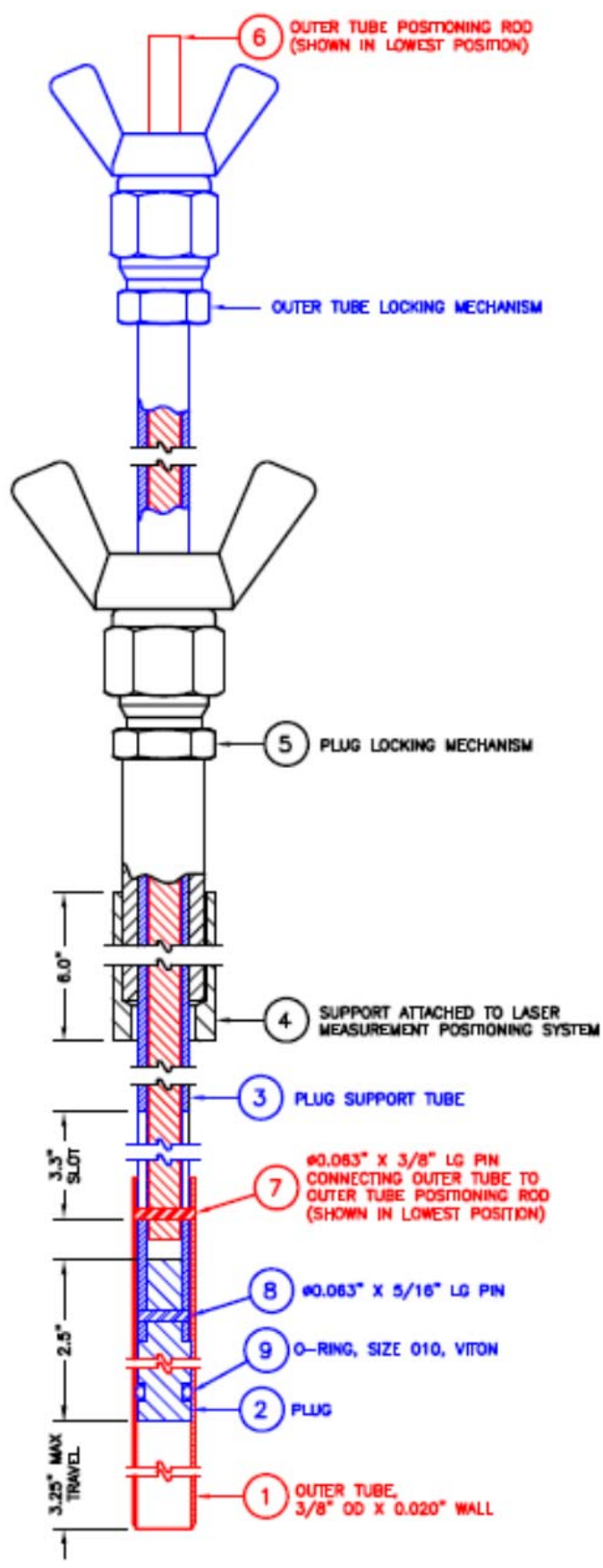

(a)

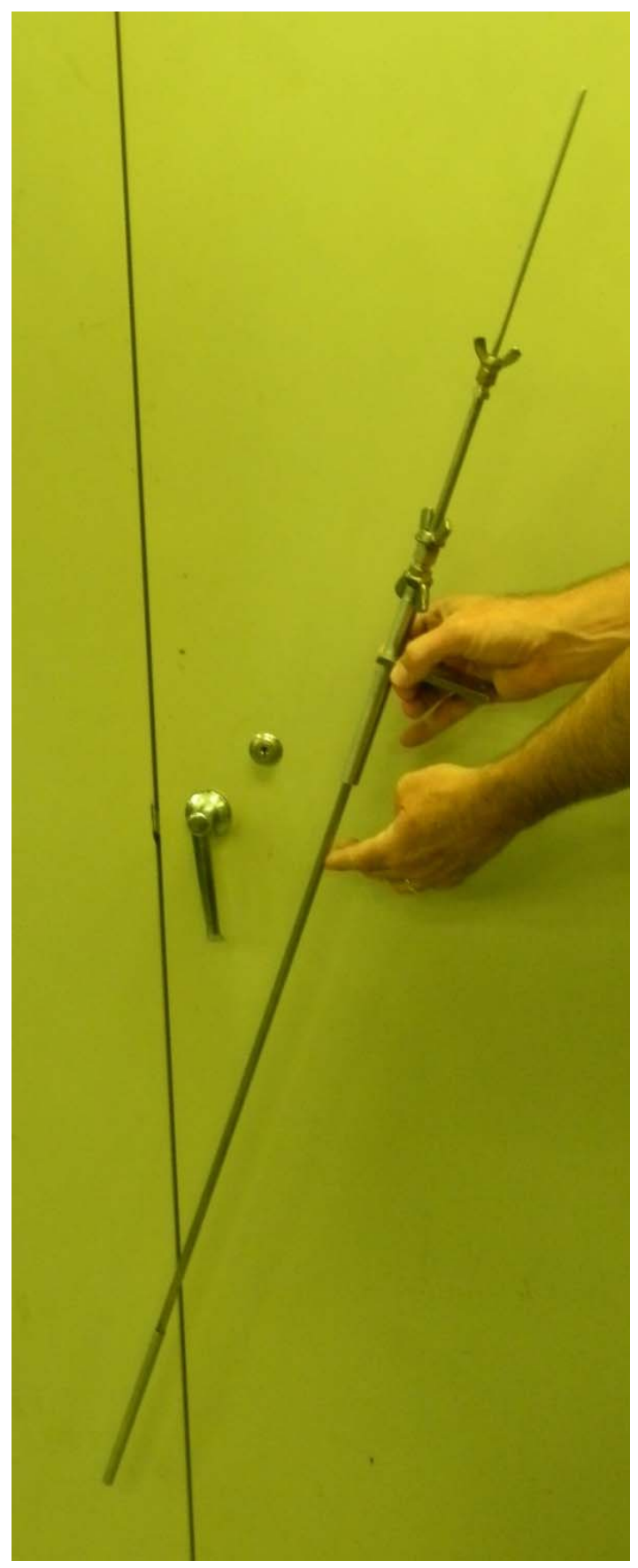

(b)

Figure 3-15. Details of the core sampler (a) Illustrated drawing, (b) actual device.

The core sampler was tested with trial solids mounds and found to extract good cores if the solids were slightly damp and the plug was mildly packed before retracting the sampler. Figure 3-16(a) shows the bottom of the sampler and Figure 3-16(b) shows a sample being taken. The sampler removes most of the solids plug targeted, however, there is always a little bit of the core left behind. The amount left behind was not quantified because of the difficulty obtaining the remnants. As can be seen in Figure 3-16(b), in most cases the overlying solids on the mound, 
assumed to be mostly gibbsite, back filled the hole as soon as the core sampler removes a plug. Trial runs indicate better than $95 \%$ of the plug is removed by the core sample. Unfortunately, when looking from the bottom it was not possible to see where a core was taken. As already mentioned, the accuracy of the placement of the sampler was estimated at $\pm 3 / 8$ inch.

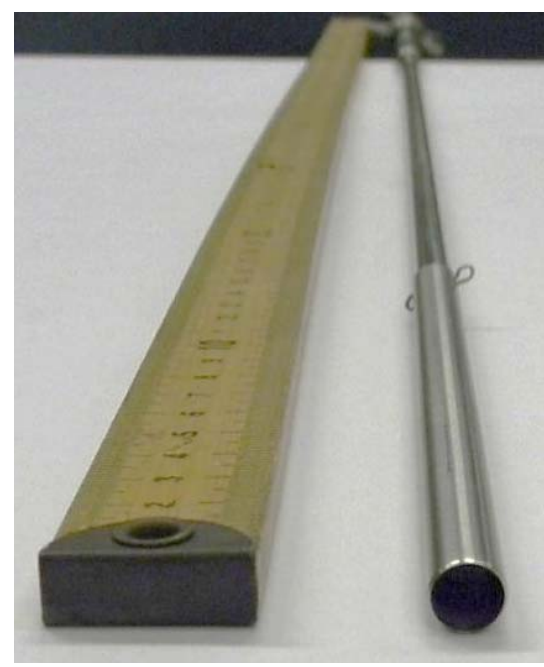

(a)

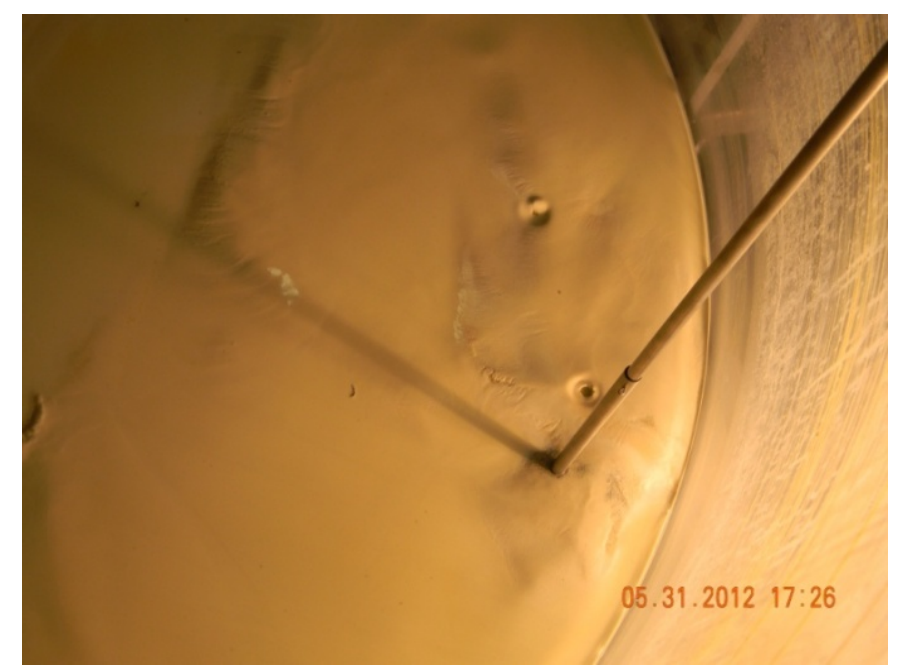

(b)

Figure 3-16. Core Sampling (a) The core end of the sampler, (b) a core being extracted during Cycle 1 of Campaign 1.

Each time heel measurements were made the heel liquid was removed to expose the mounds of accumulated solids. Unless a campaign was at its end that removed heel material was replaced. This process was demonstrated by removing the heel material through the MDT transfer tube and it was replaced in the same manner by simply reversing the flow. A peristaltic pump was connected to the transfer line to perform this process. As already mentioned above, the flowrate during the heel removal was approximately $0.8 \mathrm{gpm}$, which appeared not to disturb the accumulated solids, but it was returned at a slower rate, i.e., $0.5 \mathrm{gpm}$, to better stop at the 0.2 -inch increments when pictures were taken to determine mound volumes.

\subsection{Accumulated Solids Removal}

As the end of each campaign it was important to recover the solids left in the MDT to determine the amount of stainless steel accumulated over the Ten fill and empty cycles, especially from the two mounds. This was done in two steps:

1. Mixing the MDT vigorously to suspend all of the accumulated solids and transferring the mixture through the magnetic separator, Figure 3-3, until the heel level was too small to effectively mix.

2. Manually sweep the remaining material in the MDT with hand magnets, Figure 3-17, to collect as much of the stainless steel as possible. The hand-held magnets were made up of a wooden probe with a strong magnet at one end. The probe was inserted into a plastic sleeve to pick up the stainless steel particles. After capturing some particles the sleeve was placed into a sample bottle and the probe was removed to release the solids. The process was 
repeated until the sample bottle was full. This method worked well and when finally washing out the remaining material from the MDT no significant stainless steel was captured.

As will be explained in the Results Section, Steps 1 and 2 above were performed at the end of Campaign 1, and the process worked well, but because Step 1 suspends all the MDT solids information was lost for solids residing in the two individual mounds. For Campaign 2 only Step 2 was used in attempt to determine the stainless steel in each of the mounds and what remained in the other areas of the tank bottom.

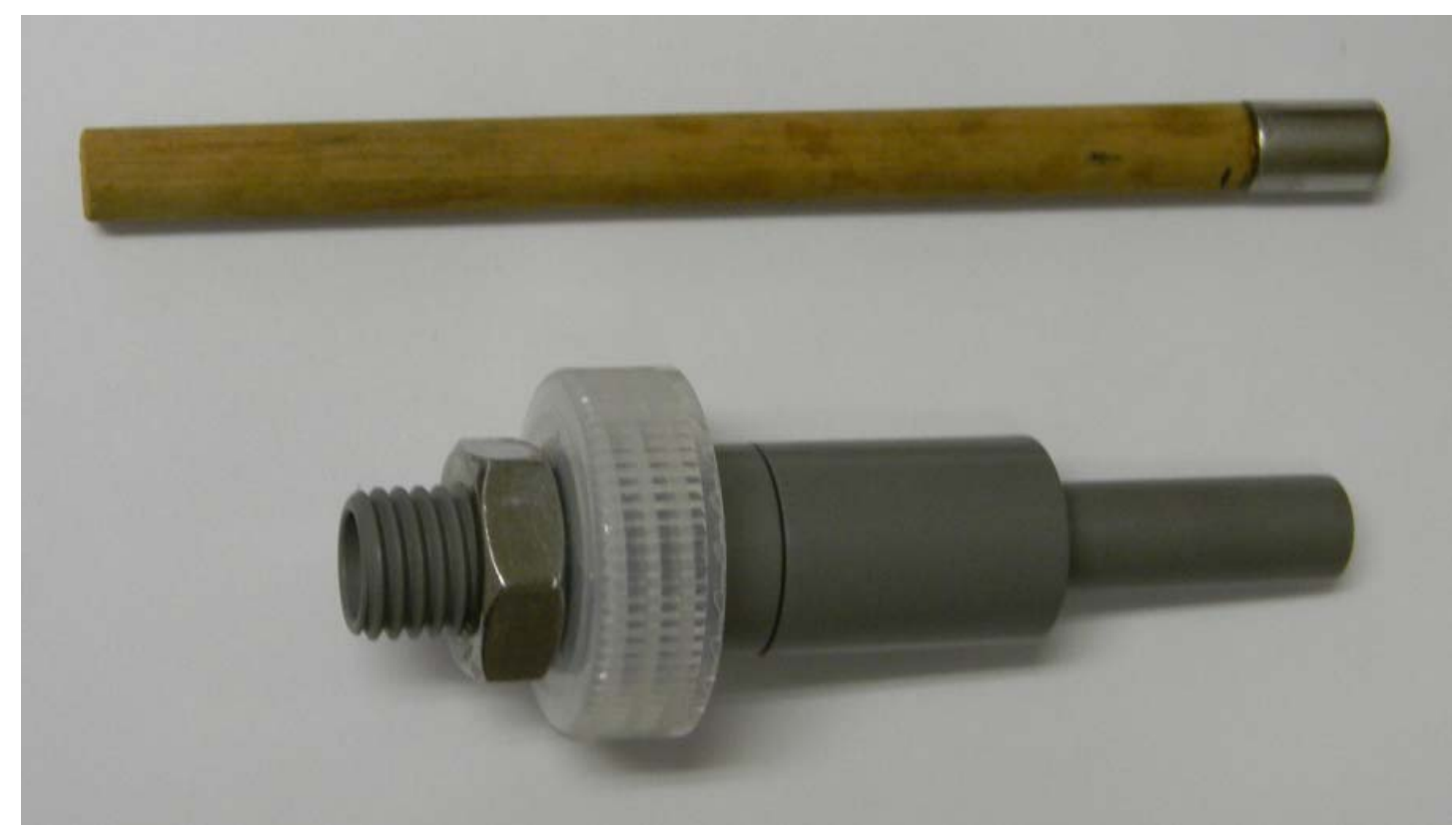

Figure 3-17. Hand-held magnet for separating stainless steel manually.

\subsection{Results and Discussion}

The results can be broken into three broad categories:

1. Operation of the test facility - Sections 4.1

2. Solids transferred out of the MDT - Section 4.2

3. Solids accumulated in the MDT - Section 4.3

The remaining sections show the results of the stainless steel mass balance, Section 4.4, and explain why the North mound was larger than the South mound, Section 4.5.

\subsection{Operation of the Test Facility}

The operation of the test facility processed flow in three phases: MDT Filling, MDT Mixing and Pump Rotation, and Batch Transfers. 


\subsubsection{MDT Filling}

To start each campaign and each cycle the MDT had to be first filled and after Cycle 1 the MDT was filled at the end of each cycle to begin the next cycle. During pretrial tests, Section 3.6, several MDT filling rates were tested in order to choose the fastest rate that would not disturb the accumulated solids contained within the heel of 3.2 inches of simulant. The flowrate chosen was the maximum attainable of $1.8 \mathrm{gpm}$ and therefore used for all fillings. In most cases the actual flowrate was only recorded in the task notebook because it was constant and the filling generally occurred after all batch transfers were complete.

\subsubsection{MDT Mixing and Pump Rotation}

Once filled the MDT was mixed with two rotating jet pumps. Table 4-1 shows all the average Campaign 1 results for each transfer batch with the key results being:

- The average jet pump velocity was $22.9 \mathrm{ft} / \mathrm{s}$ and the target was $22.4 \mathrm{ft} / \mathrm{s}$.

- The average jet pump rotation was $1.54 \mathrm{rpm}$ and the target was $1.57 \mathrm{rpm}$.

- The average batch transfer flowrate was $0.58 \mathrm{gpm}$ and the target was $0.58 \mathrm{rpm}$.

Table 4-1. Campaign 1 flowrates, velocities, and pump rotation

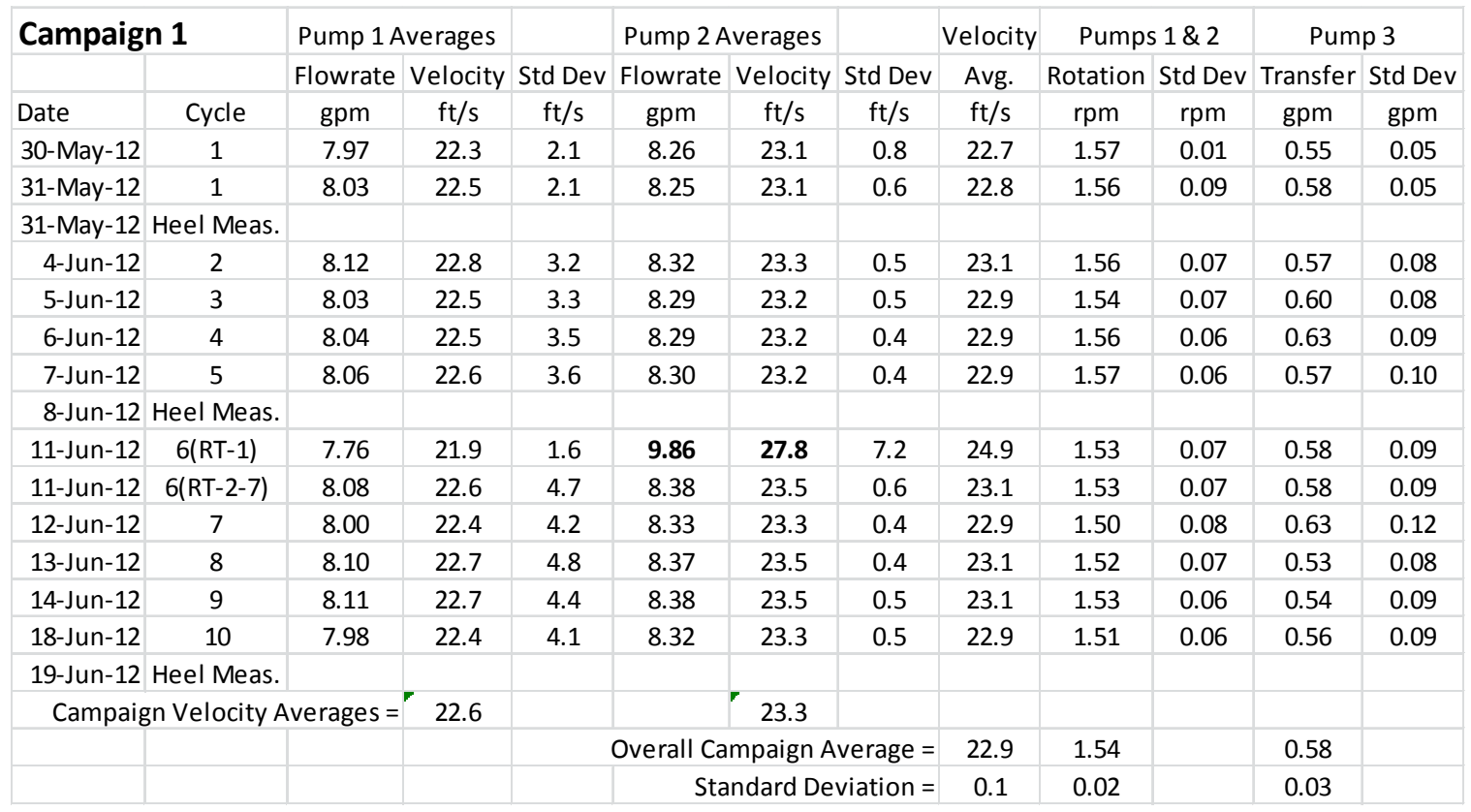

Notes on Table 4-1:

a. On 11 June during RT-1 one of the seal failed on MJP2 causing simulant to recirculate, which gave the appearance of a high velocity, i.e., $27.8 \mathrm{ft} / \mathrm{s}$. However, this was the result of the high flowrate internal to the pump as it recirculated simulant. The actual jet velocity was much less than the target causing considerably less solids to transfer for that batch transfer, which can be seen in the solids transfer data. After replacing the seal, the 
next transfer began, i.e., RT-2 and the velocity returned to what was expected, as is seen in the table.

b. The large flow/velocity fluctuations seen with MJP1, seen in both Table 4-1 and Table 4-2, are discussed later in following Section 4.1.2.1.

Table 4-2 shows all the average Campaign 2 results for each transfer batch with the key results being:

- The average jet pump velocity was $23.9 \mathrm{ft} / \mathrm{s}$ and the target was $23.5 \mathrm{ft} / \mathrm{s}$.

- The average jet pump rotation was $1.75 \mathrm{rpm}$ and the targeted was $1.72 \mathrm{rpm}$.

- The average batch transfer flowrate was $0.55 \mathrm{gpm}$ and the target was $0.58 \mathrm{rpm}$

Table 4-2. Campaign 2 flowrates, velocities, and pump rotation

\begin{tabular}{|c|c|c|c|c|c|c|c|c|c|c|c|c|}
\hline \multicolumn{2}{|c|}{ Campaign 2} & \multicolumn{2}{|c|}{ Pump 1 Averages } & \multirow[b]{2}{*}{ Std Dev } & \multicolumn{2}{|c|}{ Pump 2 Averages } & \multirow{2}{*}{ Std Dev } & \multirow{2}{*}{$\begin{array}{l}\text { Velocity } \\
\text { Avg. }\end{array}$} & \multicolumn{2}{|c|}{ Pumps $1 \& 2$} & \multicolumn{2}{|c|}{ Pump 3} \\
\hline & & Flowrate & Velocity & & Flowrate & Velocity & & & Rotation & Std Dev & Transfer & Std Dev \\
\hline 20-Jun-12 & 1 & 8.34 & 23.4 & 4.2 & 8.68 & 24.3 & 0.6 & 23.9 & 1.73 & 0.06 & 0.60 & 0.10 \\
\hline 21-Jun-12 & Heel Meas. & & & & & & & & & & & \\
\hline 22-Jun-12 & 2 & 8.23 & 23.1 & 4.1 & 8.63 & 24.2 & 0.4 & 23.7 & 1.68 & 0.08 & 0.59 & 0.08 \\
\hline 25-Jun-12 & 3 & 8.30 & 23.3 & 4.6 & 8.65 & 24.3 & 0.4 & 23.8 & 1.77 & 0.07 & 0.54 & 0.07 \\
\hline 26-Jun-12 & 4 & 8.24 & 23.1 & 5.0 & 8.67 & 24.3 & 0.4 & 23.7 & 1.76 & 0.06 & 0.54 & 0.08 \\
\hline 27-Jun-12 & 5 & 8.22 & 23.0 & 4.3 & 8.62 & 24.2 & 0.4 & 23.6 & 1.76 & 0.07 & 0.54 & 0.10 \\
\hline 28-Jun-12 & Heel Meas. & & & & & & & & & & & \\
\hline 2-Jul-12 & 6 & 8.31 & 23.3 & 4.9 & 8.71 & 24.4 & 0.4 & 23.9 & 1.77 & 0.06 & 0.58 & 0.09 \\
\hline 3-Jul-12 & 7 & 8.27 & 23.2 & 6.0 & 8.80 & 24.7 & 0.5 & 24.0 & 1.79 & 0.20 & 0.57 & 0.12 \\
\hline 5-Jul-12 & 8 & 8.40 & 23.5 & 4.9 & 8.79 & 24.6 & 0.6 & 24.1 & 1.69 & 0.38 & 0.52 & 0.10 \\
\hline 6-Jul-12 & 9 & 8.38 & 23.4 & 4.7 & 8.84 & 24.8 & 0.5 & 24.1 & 1.78 & 0.06 & 0.54 & 0.12 \\
\hline 9-Jul-12 & 10 & 8.34 & 23.4 & 4.7 & 8.87 & 24.9 & 0.5 & 24.2 & 1.77 & 0.06 & 0.52 & 0.10 \\
\hline \multicolumn{13}{|c|}{ 10-Jul-12 Heel Meas. } \\
\hline \multicolumn{3}{|c|}{ Campaign Velocity Averages = } & 23.3 & & & 24.5 & & & & & & \\
\hline & & & & \multicolumn{4}{|c|}{ Overall Campaign Average $=$} & 23.9 & 1.75 & & 0.55 & \\
\hline & & & & & \multicolumn{3}{|c|}{ Standard Deviation $=$} & 0.2 & 0.04 & & 0.03 & \\
\hline
\end{tabular}

\subsubsection{Mixing Jet Pump Fluctuation}

The mixing jet pumps (MJP) were used to mix the MDT before and during each batch transfer. Figure 4-1 shows the data for one example of the jet pumps in operation. Obviously, the flowrate for MJP1 "appears" to be very erratic with large swings for reasons unknown. This difference in pump operations was also seen in past tests using the same equipment [5-7] and the reason for such swings was not investigate primarily because the flowrate into the MDT appeared to the be even and steady. 


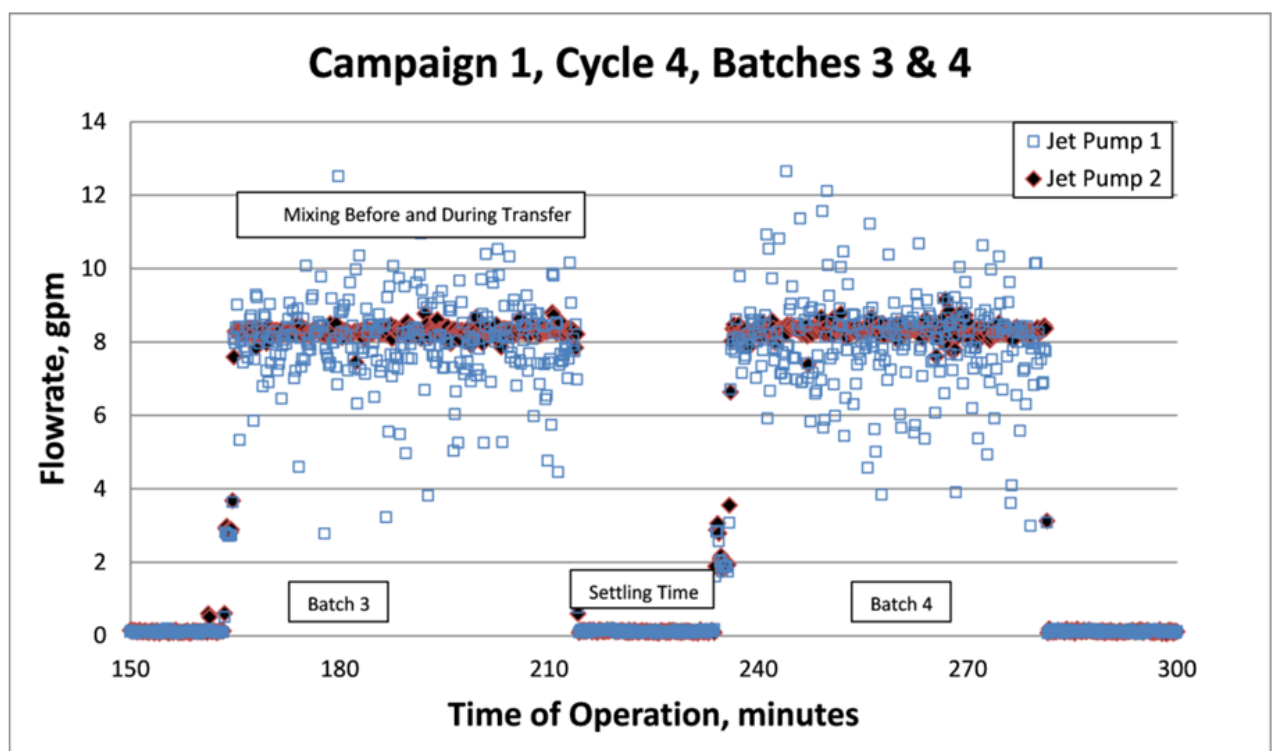

Figure 4-1. Example of the Jet Pump history during two batch transfers.

When studying the many pictures of the jets from the two MJPs the strength and stability of the jets appeared equal, for example see Figure 4-2(a) and Figure 4-2(b). While it is hard to judge from still pictures, watching the moving films show jets of similar strength that projected to a uniform distance. Both appeared to have approximately the same amount of fluctuation. The two magnetic flowmeters used to measured jet flows were located in pipe sections of different sizes. This style of flowmeter imposes a magnetic field around the flow. When a conductive liquid flows through the magnetic field, a small voltage is induced. This voltage is proportional to the velocity of the flow and is accurately measured by two electrodes mounted opposite each other inside the metering pipe. It is assumed the flowmeter exhibiting the larger fluctuation may have been affected by air or solids that influenced the sensing electrodes, but this issue was not investigated; however, these large fluctuations were not present during calibration in a separate calibration loop, therefore, it was not an instrument problem. The large fluctuations seen in the MJP1 data do not appear as the jets exit the pumps, as mentioned above. The large pressure drop and long pathway to the jet at the end of the jet pump probably dampens those fluctuations, if they were hydraulically real.

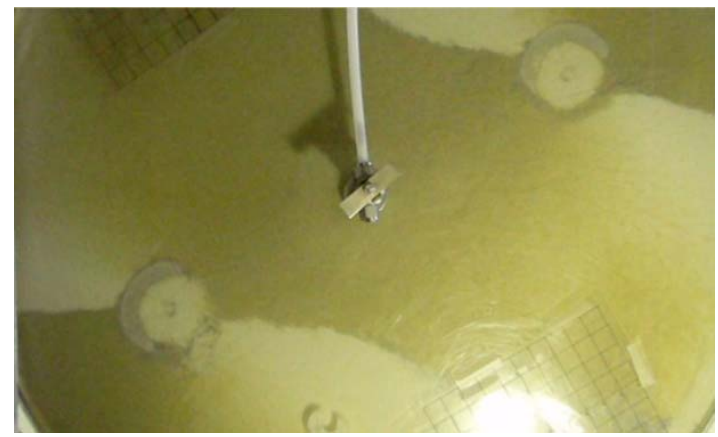

(a)

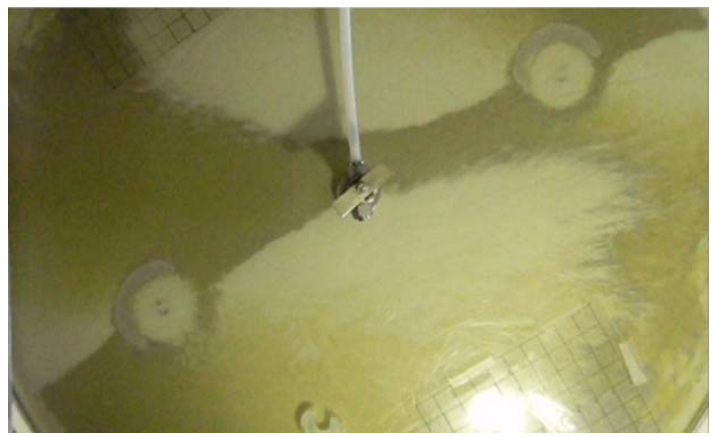

(b)

Figure 4-2. A typical Campaign 2 jet pump operation rotating at $1.74 \mathrm{rpm}$.

The time difference between (a) and (b) of 3 to 4 second in time. The jets appear to be uniform in length and width and both appear to be equally stable. 


\subsubsection{Batch Transfers}

Figure 4-1 and Figure 4-3 are comparable in that they are for both the same transfer histories and shown over the same operational time period. After the MDT was mixed for 5 turnovers of its volume, then a batch transfer began. During both the MDT mixing and batch transfer the MJPs continued to mix and rotate. For both campaigns the mixing and transferring process was the same and Figure 4-3 is used to explain the process:

1. During the period from 150 to 160 minutes the MJP were not mixing and rotating, which allowed the MDT contents to settle while waiting to begin the next batch transfer, Batch 3 . This period was approximately 20 -minutes long, but only 10 minutes are shown here.

2. From 150 to 190 minutes the transfer pump was in recirculation. This was done to minimize solids plugging in the transfer lines and pump.

3. From 160 to 210 minutes the MJP were mixing and rotating during the period in order to mix the MDT contents five volume turnovers and when transferring to the appropriate Receipt Tank (RT).

4. From 190 to 210 minutes the simulant was transferred from the MDT to the RT.

5. From 210 to 235 minutes the MJP was not mixing and rotating, as in Step 1, while waiting to repeat the entire process for Batch 4.

As in previous testing [5-7] the transfer line leading to the transfer pump was a 3/8-inch tube with a 0.065 -inch wall giving a transfer velocity of $3.9 \mathrm{ft} / \mathrm{s}$, which is the same as the full scale system. From the transfer pump discharge to the receipt tank the transfer line was $1 / 4$-inch tube with a 0.035 -inch wall, giving the transfer velocity as $7.3 \mathrm{ft} / \mathrm{s}$, which is slight higher that the full-scale of $6.1 \mathrm{ft} / \mathrm{s}$. At both scales that velocity was not expected to allow solids to settle while being transferred.

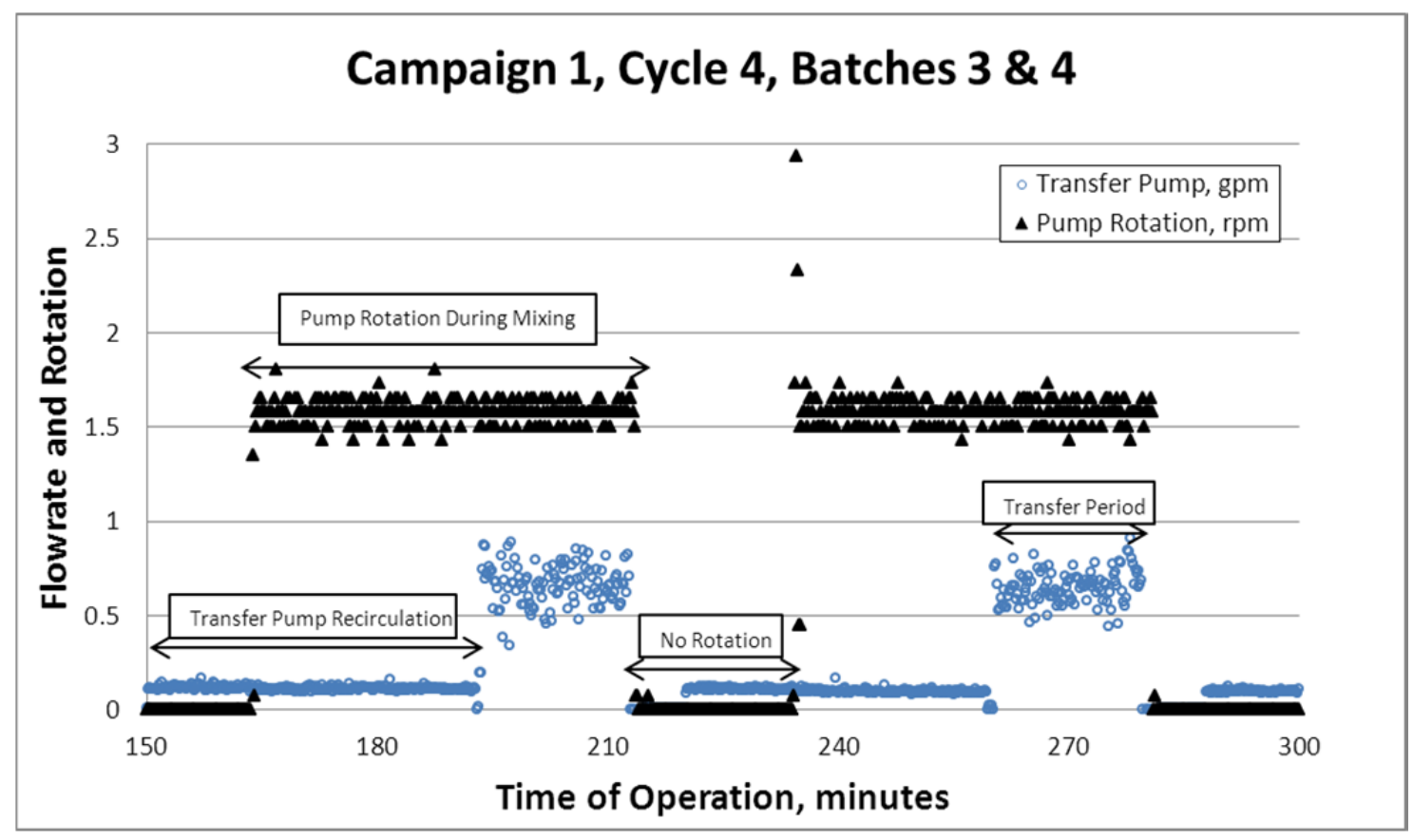

Figure 4-3. Example of the transfer pump and jet pump rotational histories. 
From Figure 4-3 it can be seen that flow through the transfer pump was generally not stopped. When it was not transferring a batch it was put into a recirculation mode at a low flowrate, e. g. $0.1 \mathrm{gpm}$, to minimize pluggage of the fast settling solids.

\subsubsection{Temperature}

Testing was performed at room temperature, which of course changes during the day as the environmental temperature changes. The temperature of MDT contents will also change to represent the pump heat dissipation. Because the jet pump mixing energy was held constant throughout all seven batch transfers the simulant temperature should increase as the tank level drops, which left less volume of liquid to remove the heat. Figure 4-4 shows the measured results, which were obtained using a hand-held thermistor, calibrated uncertainty of $\pm 0.2^{\circ} \mathrm{C}$, Table $4-2$, that was submerged into the mixing MDT simulant during each batch. The data for the campaigns are grouped by transfer batch because for the same batch number the simulant volume of the MDT was the same and each datum point is the average from all 10 cycles for each campaign. The difference between the campaigns from batch to batch is basically due the difference in the room temperature. Campaign 2 was generally hotter than Campaign 1 due to the increasing outside temperature during the period from May to July. Note, during the entire test period the laboratory air conditioning system was being replaced and not working making the building temperature totally dependent upon the environment.

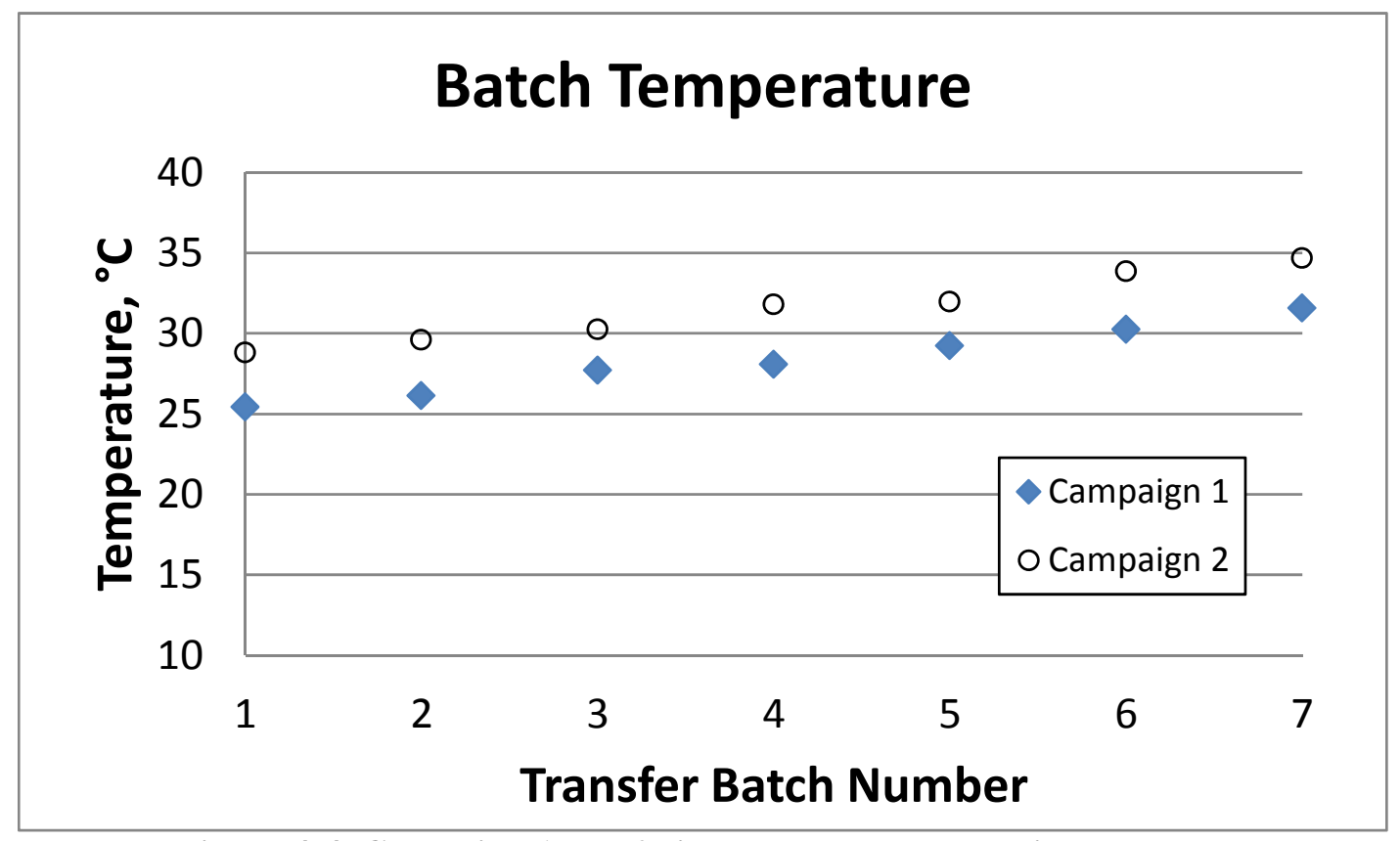

Figure 4-4. Campaign 1 and 2 simulant temperatures in the MDT. 


\subsection{Solids Transferred out of the MDT}

During a batch transfer the slurry contained concentrations of all four undissolved solids of the simulant, two of which were quantified: Sand and Stainless Steel. The sand was roughly estimated from the sand that settled in the seven receipt tank and the stainless steel was estimated by using a magnetic to separate, and subsequently measure, those solids as the slurry was transferred to the receipt tanks. The next two sections discuss the results of those estimations.

\subsubsection{Sand Transfer}

To estimate the amount of sand transfer in each batch transfer, seven for each cycle, the sand height was measured in the appropriate Receipt Tank (RT). This was done after waiting 20 minutes from the time a transfer was complete. Of course this was a rough measurement because the sand settled with the other solids, i.e., Zirconia and gibbsite, and not always evenly in the settling tube portion of a RT. However, as discussed in the trial test runs, Section 3.1, the settled sand height in a full simulant test was approximately the same as the sand settling when it was the only solids suspended in simulant supernatant, Figure 3-1. Therefore, the measure of sand from the transfers was expected to be reasonably close to the actual sand transferred. Figure 4-5 shows typical settling heights.

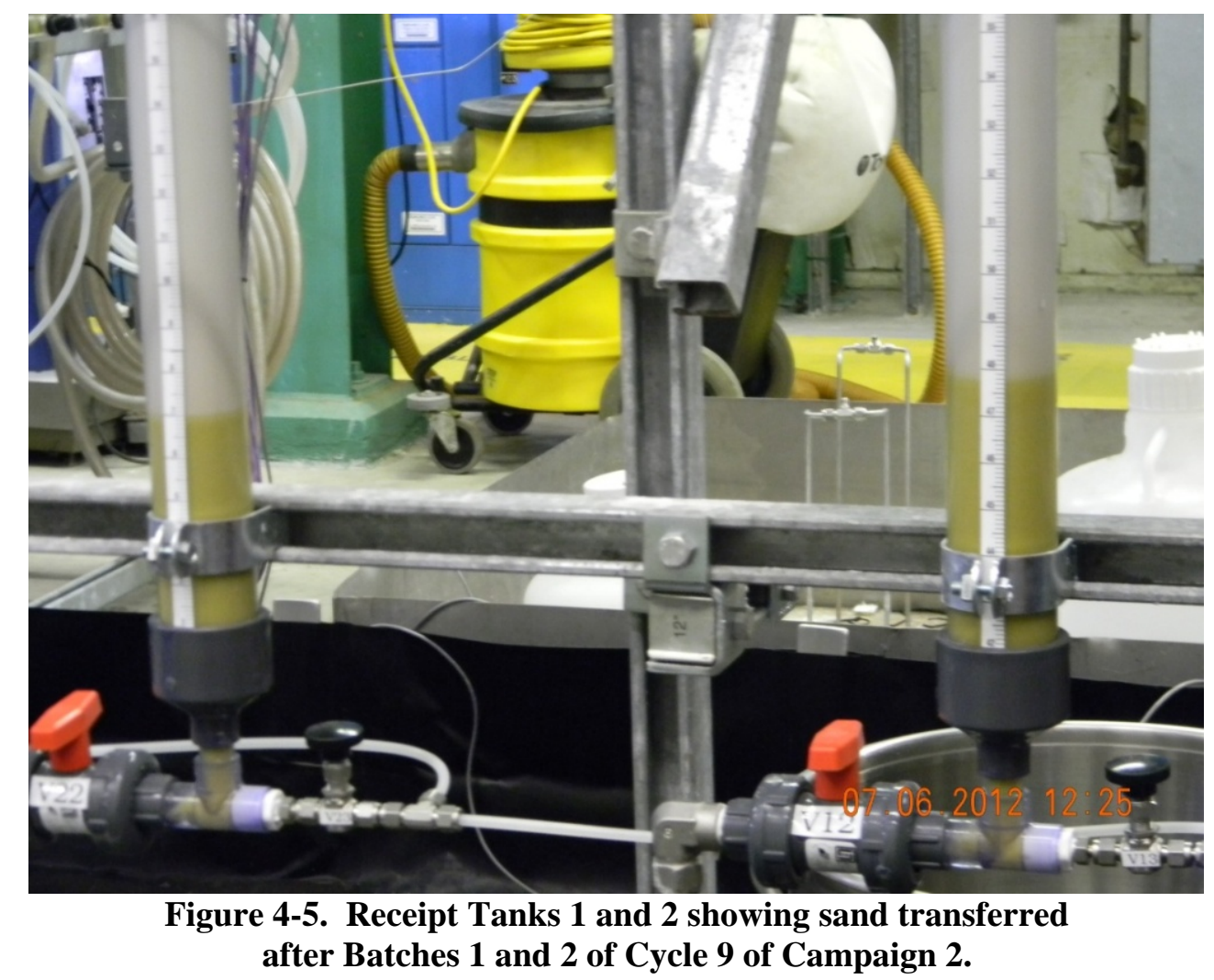

The sand settling results are shown in Figure 4-6 and Figure 4-7. After about the second cycle the sand no longer accumulated significantly in the MDT and the transferred amount began to 
level out. The fact that the results surpass $100 \%$ probably indicates that some of the other solids actually became trapped with the sand, which led to slightly higher readings, but in general the measurement of transferred sand is fairly good. An attempt was made to record the zirconia levels in the RTs. The zirconia was a very light grey in color, while the gibbsite had a more whitish tone. A few times it was possible to make the distinctions between those two solids in the RTs but for most transfers it was impossible to see the difference. There were not sufficient data to determine transferred zirconia volumes.

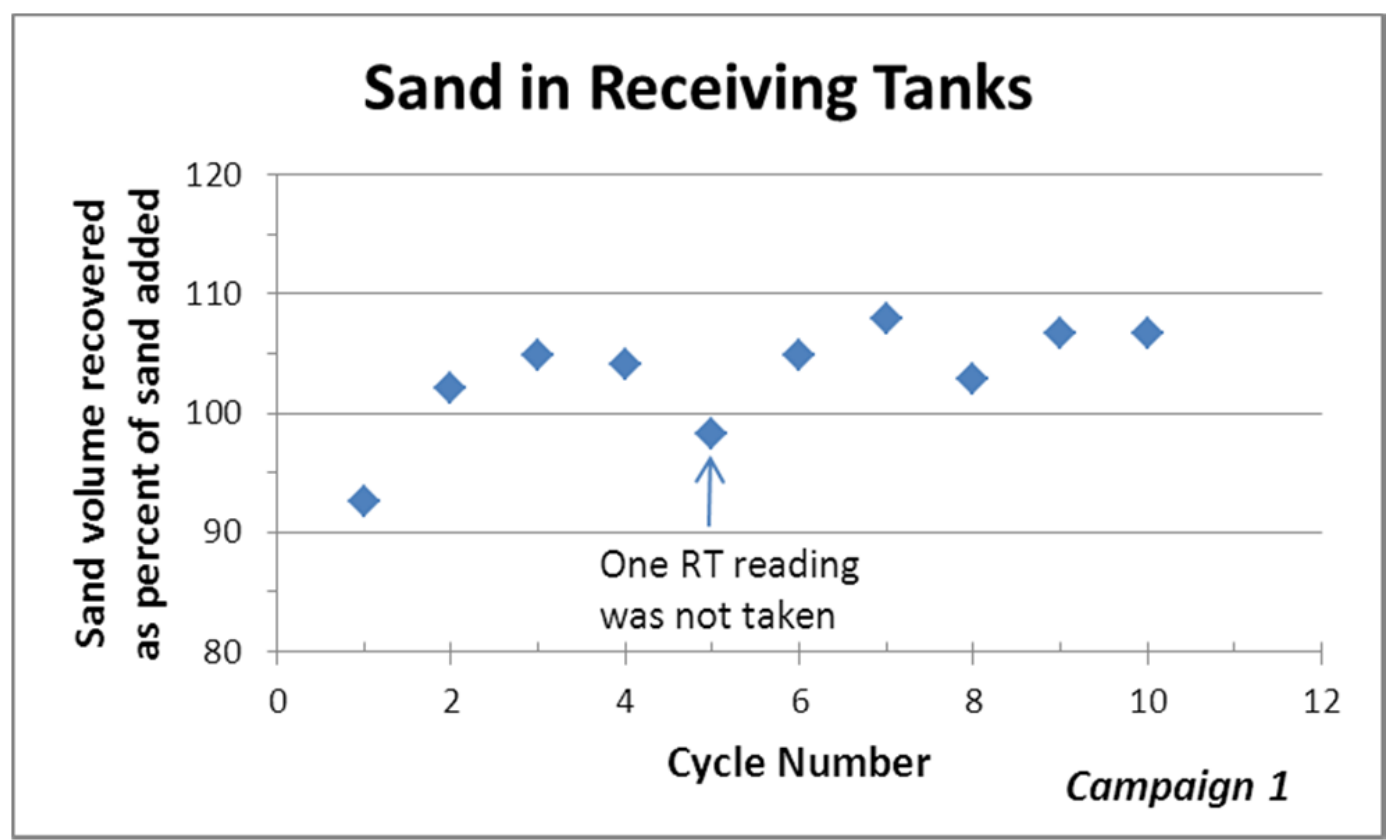

Figure 4-6. Sand transferred during Campaign 1.

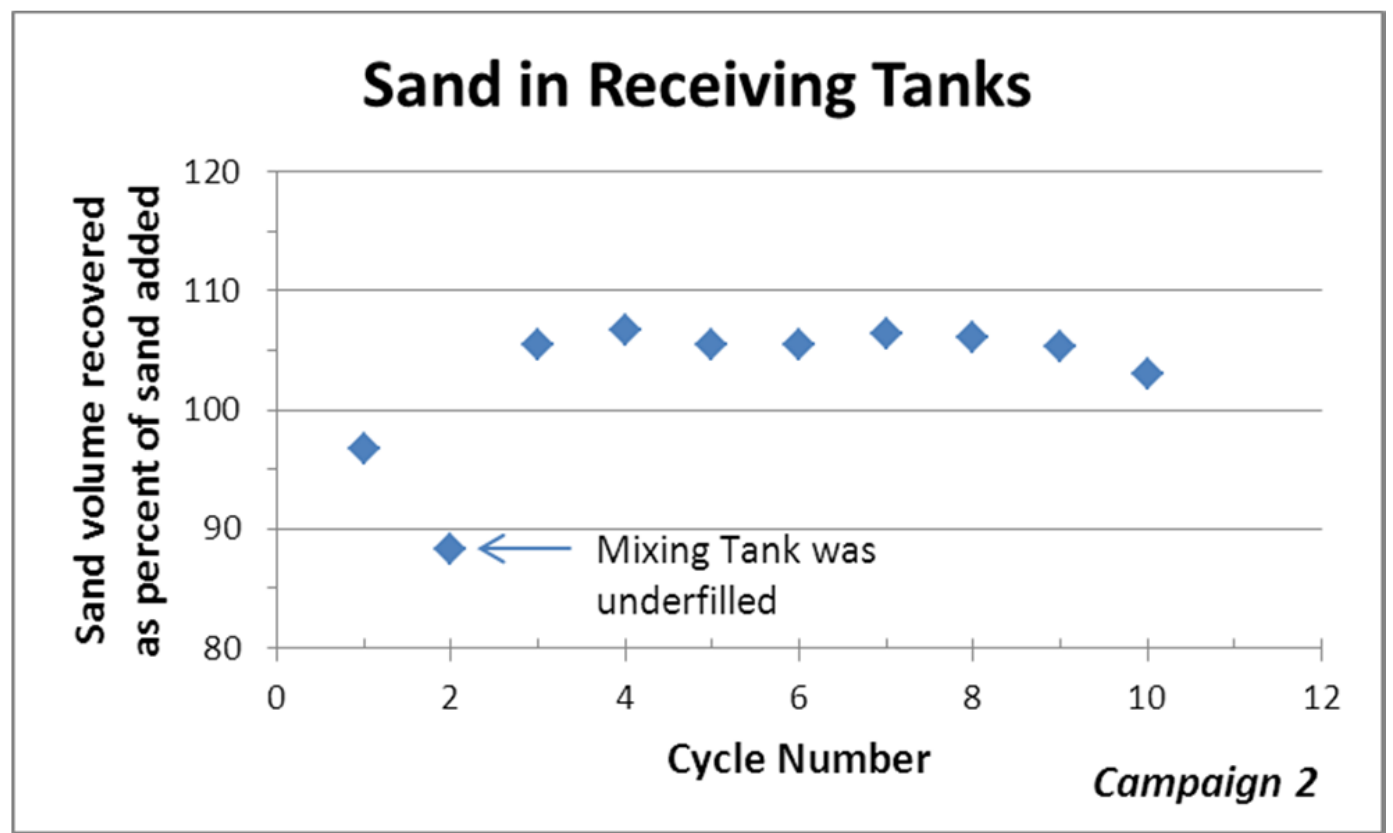

Figure 4-7. Sand transferred during Campaign 2. 


\subsubsection{Stainless Steel Transfer}

Based on the requirement of an undissolved solids loading of $100 \mathrm{~g} / \mathrm{l}$, Table 2-3, a scaled cycle batch size of 392 liters (or 103.6 gal. Table 2-1), and the customer agreement that stainless steel (SS) particles make up $1 \mathrm{wt} \%$ of the solids, as shown in Table 2-3, then the first cycle of both campaigns was loaded with 392 grams of SS. Due to the requirement to leave a 3.2-inch heel (68 liters) in the MDT (to represent the 72-inch heel planned for the full-scale tank after each fill and empty cycle) the subsequent refill volume was only 324 liters, which required a charge of 324 grams of SS. Over the entire campaign the amount of SS added to the MDT was 3,308 grams, which should have been the maximum SS mass that could be recovered. The amount of stainless steel recovered was expected to be slightly less than this value due to losses throughout the campaign; however, the mass of nominal stainless steel recovered was larger, which will be discussed later in the section on the SS mass balance, Section 4.4. Briefly stated, the collected stainless steel was always somewhat contaminated with other substances, so the reported mass reflected more than just stainless steel.

For Campaign 1, Table 4-3, a total of 3282 grams of nominal SS was measured to have transferred out of the MDT during the 70 transfers over the 10 cycles of operation with an average transfer of 340 grams per cycle (this average is explained in the following paragraph). As previously stated a mass of 392 grams was initially charged to the MDT. By assuming that the mass loss of stainless steel from the tank was proportional to total volume transfer, an estimated 68 grams of stainless steel remained in the tank at the end of each cycle. Before the start of each new cycle 324 grams of stainless steel were added to bring the estimated total inventory to 392 grams. As a result of this reasoning the actual mass of stainless steel transferred was compared to 392 grams as a percentage in the last column of Table 4.3 (and Table 4-4 for Campaign 2).

Note that in Table 4-3, the average transfer does not include Cycles 3 and 6 which transferred less SS due to Magnetic Separator being accidentally left out of the flow stream; therefore, the 340 grams is slightly larger than $1 / 10^{\text {th }}$ of the total actual mass transferred. Furthermore, because a 68-liter remained after each transfer only 324 liters are transferred each cycle with an initial concentration of 324 grams. This means that not only does most of the SS in the mixed MDT leave during the transfer, but the heel is depleted of SS with time. 
Table 4-3. Stainless steel transferred during Campaign 1

\begin{tabular}{|c|c|c|c|c|c|c|c|c|c|}
\hline \multirow[b]{2}{*}{ Cycle } & \multicolumn{7}{|c|}{ Mass of SS Batch Transfer to Receipt Tanks (g) } & \multicolumn{2}{|c|}{ SS Transferred } \\
\hline & 1 & 2 & 3 & 4 & 5 & 6 & 7 & gram & Percent (1) \\
\hline 1 & 47.6 & 44.2 & 44.8 & 45.6 & 44.1 & 41.7 & 15.7 & 283.7 & $72 \%$ \\
\hline $2(2)$ & 51.5 & 47.0 & 47.9 & 46.3 & 47.5 & 52.3 & 11.8 & 304.2 & $78 \%$ \\
\hline 3 & 51.4 & (3) & 37.5 & 54.8 & 55.8 & 57.5 & 19.0 & 276.1 & $70 \%$ \\
\hline 4 & 55.5 & 51.8 & 53.5 & 49.9 & 49.6 & 52.6 & 19.4 & 332.4 & $85 \%$ \\
\hline 5 & 50.9 & 49.5 & 51.1 & 51.5 & 47.3 & 46.9 & 17.1 & 314.3 & $80 \%$ \\
\hline $6(5)$ & 23.9 & 66.4 & (4) & 67.2 & 74.7 & 54.7 & (4) & 286.9 & $73 \%$ \\
\hline 7 & 56.1 & 57.6 & 62.0 & 58.6 & 67.7 & 50.6 & 14.6 & 367.4 & $94 \%$ \\
\hline 8 & 53.8 & 54.2 & 65.0 & 61.1 & 59.1 & 50.3 & 17.9 & 361.4 & $92 \%$ \\
\hline 9 & 61.1 & 58.7 & 56.6 & 54.8 & 59.1 & 62.0 & 18.0 & 370.5 & $95 \%$ \\
\hline \multirow[t]{4}{*}{10} & 64.2 & 53.5 & 56.7 & 63.0 & 69.9 & 58.9 & 18.6 & 384.9 & $98 \%$ \\
\hline & & & & & \multicolumn{3}{|c|}{ Averages $(6)=$} & 340 & $87 \%$ \\
\hline & & & & \multicolumn{4}{|c|}{ Standard Deviation $=$} & 36 & $9 \%$ \\
\hline & & \multicolumn{6}{|c|}{ Total SS transferred in Campaign $1=$} & 3281.6 & \\
\hline \multicolumn{10}{|l|}{ Notes: } \\
\hline \multicolumn{10}{|c|}{ (1) Percent Transferred is based on $100 \mathrm{~g} / \mathrm{l}$ of the 392 liters full cycle batch } \\
\hline \multicolumn{10}{|c|}{ (2) Cycle 2, Batch 7: Magnet left out of Magnetic Separator for $\sim 2$ minutes } \\
\hline \multicolumn{10}{|c|}{ (3) Cycle 3, Batch 2: Magnet left out of Magnetic Separator } \\
\hline \multicolumn{10}{|c|}{ (4) Cycle 6, Batches 3 and 7: Magnet left out of Magnetic Separator } \\
\hline \multicolumn{10}{|c|}{ (5) Cycle 6, Transferred more simulant than target: plugged sight glass } \\
\hline \multicolumn{10}{|c|}{ (6) Cycles 3 and 6 are not included in the averages, see Note (3) and (4) } \\
\hline
\end{tabular}

This is further illustrated in Figure 4-8, which shows the batch-to-batch transfer amounts were fairly constant throughout each cycle. Note that Batch 7 was a partial batch by design; therefore, the amount of solids transferred was less; however, the small size batch doesn't fully explain the lower amount of stainless steel transferred because in Figure 4-9 the data of Figure 4-8 are shown as the concentration transferred and Batch 7 is still low. 


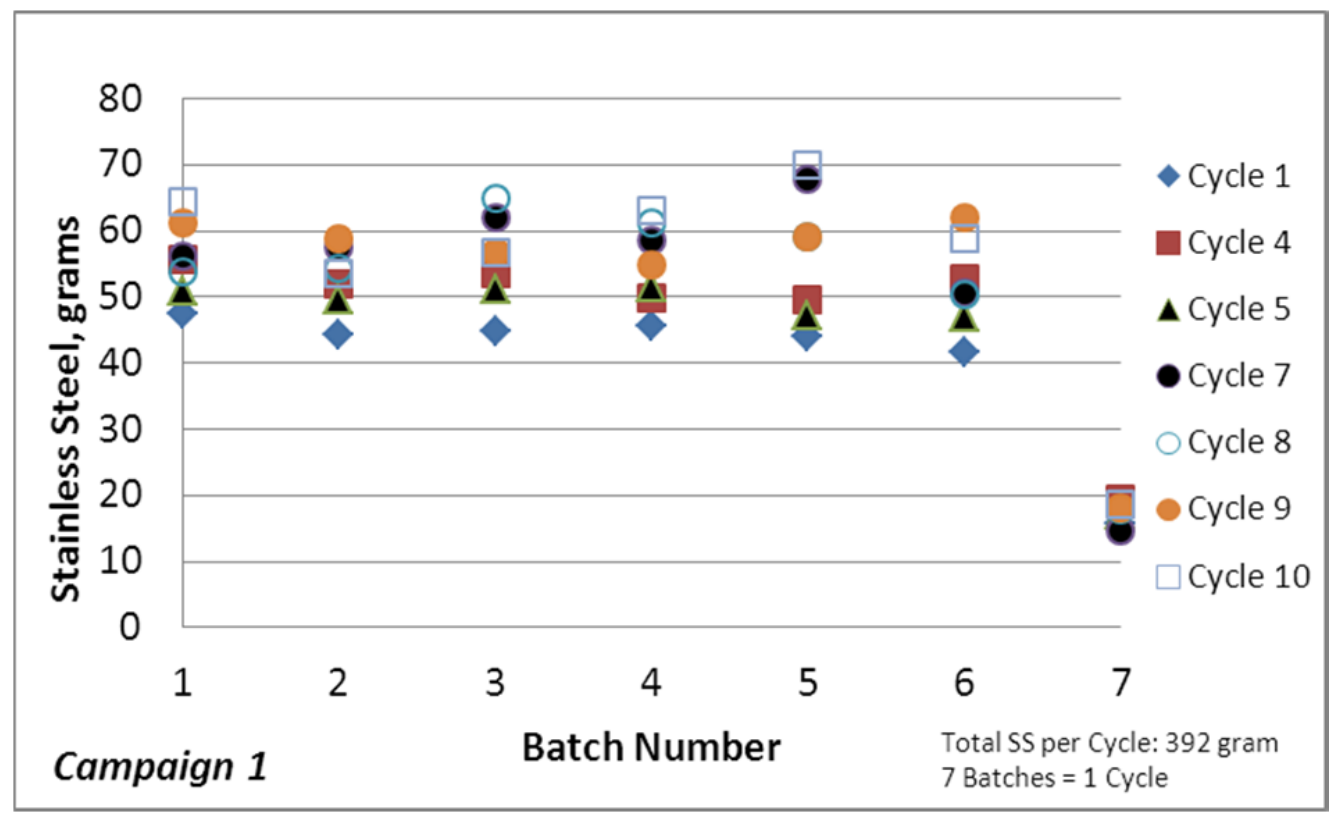

Figure 4-8. Batch to batch SS transferred during Campaign 1.

The cycle shown are expected to be a good representation of the transfer capacity of the system. Note, Batch 7 was the partial batch and therefore was smaller and to better show the batch-to-batch trends the data from Cycles 2, 3, and 6 were not included because of the Magnetic Separator being accidentally left out of the transfer line, see Notes 2, 3, and 2 in Table 4.3.

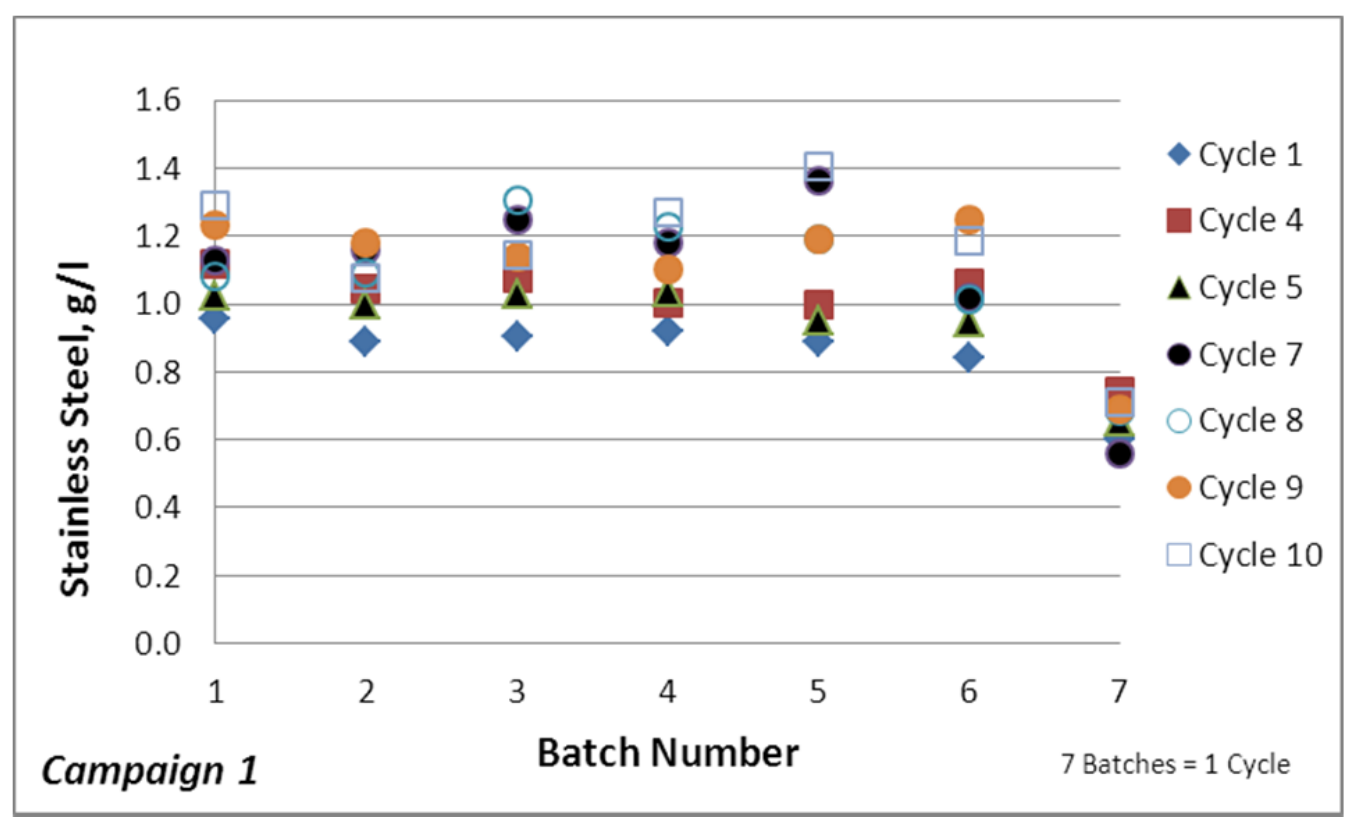

Figure 4-9. Batch to batch SS transferred during Campaign 1.

The cycle shown are expected to be a good representation of the transfer capacity of the system. Note, Batch 7 was the partial batch and therefore was smaller and to better show the batch-to-batch trends the data from Cycles 2, 3, and 6 were not included because of the Magnetic Separator being accidentally left out of the transfer line, see Notes 2, 3, and 2 in

\section{Table 4.3.}


Previous batch transfer work [see Fig. 12 of Ref. 7] has shown that the batches at the lowest tank levels result in less solids transferred, probably because at the lowest levels the thin shallow slurry pool left in the tank is harder to thoroughly mix due to the larger influence of the boundaries. Furthermore, for Figure 4-9 note that because the MDT begins with a simulant batch of 1 gram/liter (i.e., 392 grams/392 liters), the data for initial cycles transferred a concentration slightly below $1.0 \mathrm{~g} / \mathrm{l}$, but as the campaign continued the concentration increased to above $1.0 \mathrm{~g} / 1$. Figure 4-10 shows these data, but for entire cycles, based on percentage transferred. Neglecting the outliers, the transferred SS reaches approximately $90 \%$ of the starting value of SS at the beginning of a cycle. Those outliers, e.g., cycles 3 and 6 , had problems with the SS collection system, thus compromising the results for those cycles. On the average the cycle-to-cycle transfer of SS was $87 \%$, which had a standard deviation of $9 \%$, Table 4-3.

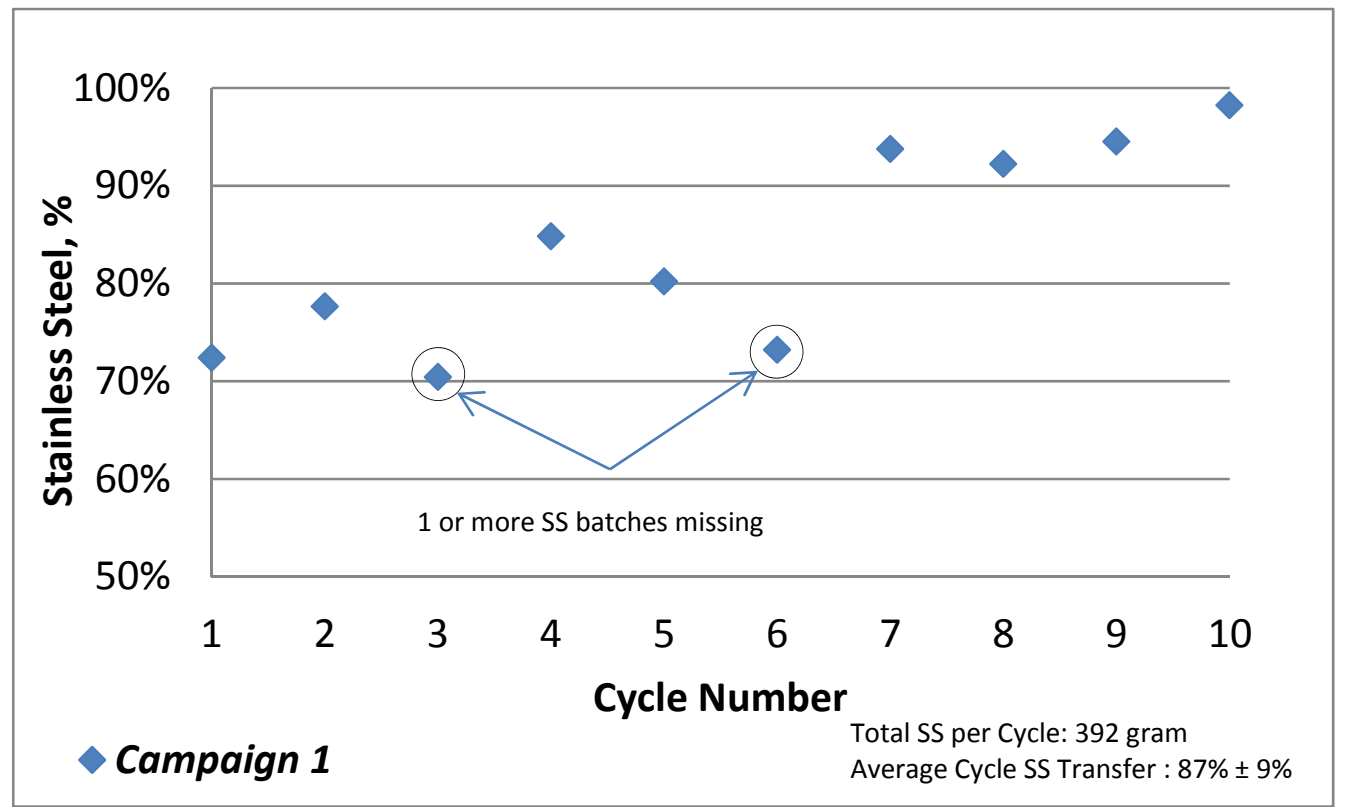

Figure 4-10. Shows the results of Figure 4-8 for each cycle.

That is, all seven batches per cycle were summed and normalized to the full cycle batch of SS available, i.e. 392 grams. Here it is clear that accumulation of the heavy SS particles does occur, but reaches a maximum at around the 7 th cycle.

Campaign 2, Table 4-4, shows a similar trend as Campaign 1. Because there were fewer problems during Campaign 2 from the lessons learned from the first campaign; therefore, the experimental data are more complete. For this second campaign, while the amount of SS used was exactly the same, the total mass of SS transferred was slightly higher, i.e., 3469 grams during the 70 transfers over the 10 cycles of operation. The average transfer of 346 grams versus the 340 grams for each cycle and the principal reason for the higher transferred amount was the higher jet pump velocity and faster pump rotation, i.e., from $22.9 \mathrm{ft} / \mathrm{s} /$ nozzle and $1.75 \mathrm{rpm}$ to 23.9 $\mathrm{ft} / \mathrm{s} /$ nozzle and $1.54 \mathrm{rpm}$, Table 4-1 and Table 4-2. That is, the higher the MJP energy the more the solids could be suspended and transferred. As for Campaign 1, for Campaign 2 most of the SS in the mixed MDT leaves during the transfer, as depicted in Figure 4-11, and it shows the batch-to-batch transfer amounts were fairly constant throughout each cycle. Figure 4-12 show the same information but based on concentration. As explained for Figure 4-9, in Figure 4-12 
because the MDT begins with a simulant batch of 1 gram/liter (i.e., 392 grams/392 liters), the data for initial cycles transfer a concentration slightly below $1.0 \mathrm{~g} / 1$, but as the campaign continued the concentration increased to above $1.0 \mathrm{~g} / \mathrm{l}$. Figure 4-13 shows these data, but for entire cycles, based on percentage transferred. Neglecting Cycle 2, which began with low initial volume in the MDT causing a slight lower end result, Campaign 2 reached $90 \%$ cycle transfers after only three cycles. This basically indicates that after approximately three cycles the solids that will accumulated on the tank bottom have reached a maximum value. From Cycle 3 to 10 the amount of SS particles leaving the MDT was fairly constant. The mounds of accumulated solids still change slightly in size and shape but there does not appear to be further growth, as will be seen from the photographic evidence shown in the next section.

Table 4-4. Stainless steel transferred during Campaign 2

\begin{tabular}{|c|c|c|c|c|c|c|c|c|c|}
\hline \multirow[b]{2}{*}{ Cycle } & \multicolumn{7}{|c|}{ Mass of SS Batch Transfer to Receipt Tanks (g) } & \multicolumn{2}{|c|}{ SS Transferred } \\
\hline & 1 & 2 & 3 & 4 & 5 & 6 & 7 & gram & Percent (1) \\
\hline 1 & 43.1 & 43.8 & 49.2 & 50.1 & 49.1 & 43.7 & 14.9 & 294.0 & $75 \%$ \\
\hline 2 & 53.0 & 49.8 & 46.3 & 51.4 & 51.9 & 22.7 & (2) & 275.2 & $70 \%$ \\
\hline 3 & 61.6 & 57.0 & 54.6 & 53.5 & 51.5 & 52.1 & 16.1 & 346.4 & $88 \%$ \\
\hline 4 & 65.1 & 60.8 & 56.0 & 54.1 & 52.4 & 53.6 & 17.7 & 359.7 & $92 \%$ \\
\hline 5 & 62.8 & 60.2 & 59.2 & 53.5 & 49.3 & 49.6 & 18.7 & 353.4 & $90 \%$ \\
\hline 6 & 65.0 & 59.0 & 56.9 & 53.0 & 51.4 & 50.9 & 17.6 & 353.7 & $90 \%$ \\
\hline 7 & 61.2 & 56.8 & 53.7 & 55.8 & 57.0 & 56.0 & 19.3 & 359.7 & $92 \%$ \\
\hline 8 & 63.4 & 61.8 & 59.3 & 58.2 & 59.4 & 52.2 & 18.1 & 372.5 & $95 \%$ \\
\hline 9 & 69.5 & 61.2 & 58.6 & 56.9 & 61.6 & 50.7 & 30.7 & 389.2 & $99 \%$ \\
\hline \multirow[t]{4}{*}{10} & 58.3 & 54.6 & 60.2 & 59.1 & 62.1 & 54.0 & 17.1 & 365.4 & $93 \%$ \\
\hline & & & & & & \multicolumn{2}{|c|}{ Averages $=$} & 346 & $88 \%$ \\
\hline & & & & \multicolumn{4}{|c|}{ Standard Deviations = } & 40 & $10 \%$ \\
\hline & & & \multicolumn{5}{|c|}{ SS transferred in Campaign $2=$} & 3469.0 & \\
\hline \multicolumn{10}{|l|}{ Notes: } \\
\hline \multicolumn{10}{|c|}{ (1) Percent Transferred is based on $100 \mathrm{~g} / \mathrm{l}$ of the 392 liters full cycle batch } \\
\hline \multicolumn{10}{|c|}{ (2) Cycle 2: MDT started accidentally underfilled and was not noticed until } \\
\hline \multicolumn{10}{|c|}{ the 1st transfer to RT1 was filled. This resulted in a partial RT6 and no RT7. } \\
\hline
\end{tabular}




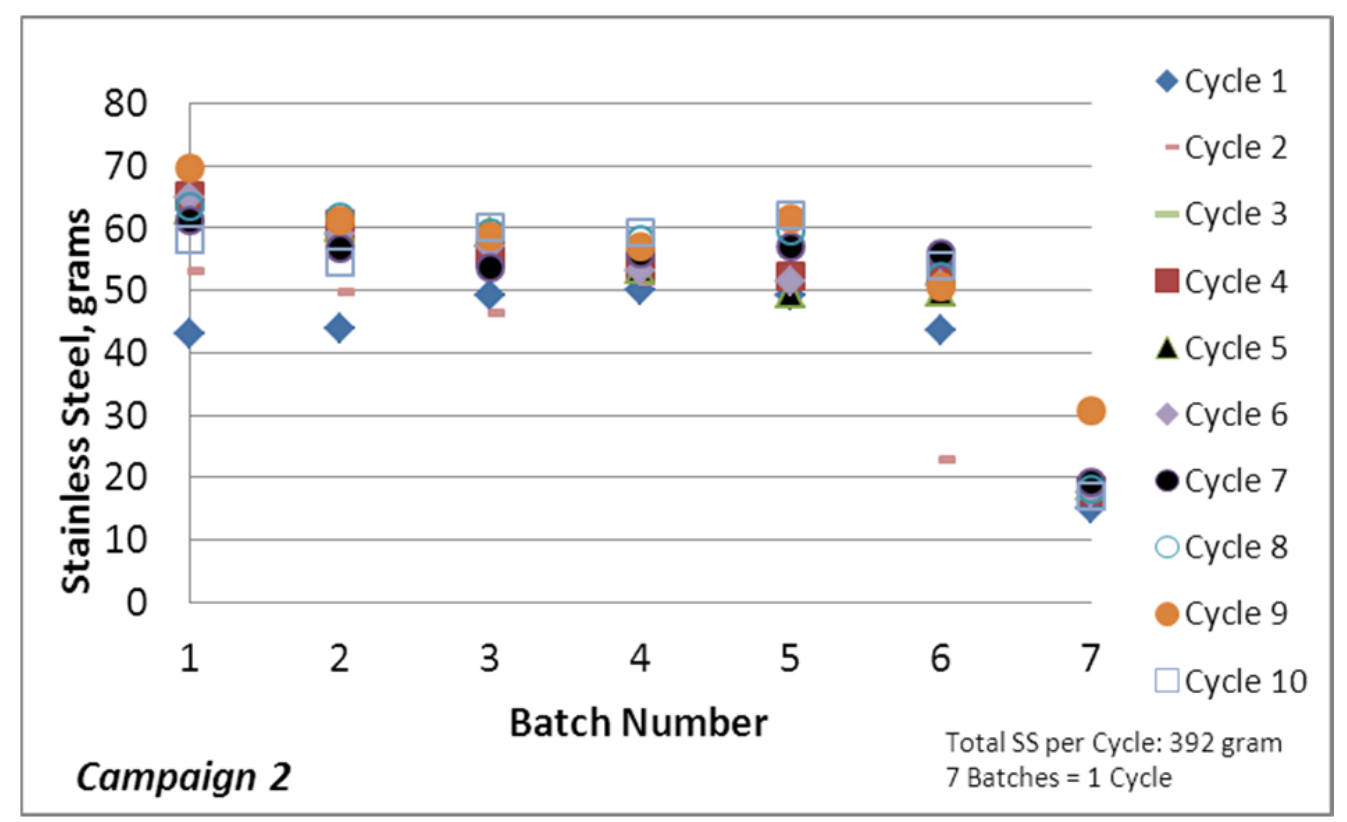

Figure 4-11. Batch to batch SS transferred during Campaign 2.

The cycle shown are expected to be a good representation of the transfer capacity of the system. Note, Batch 7 was the partial batch and therefore was smaller.

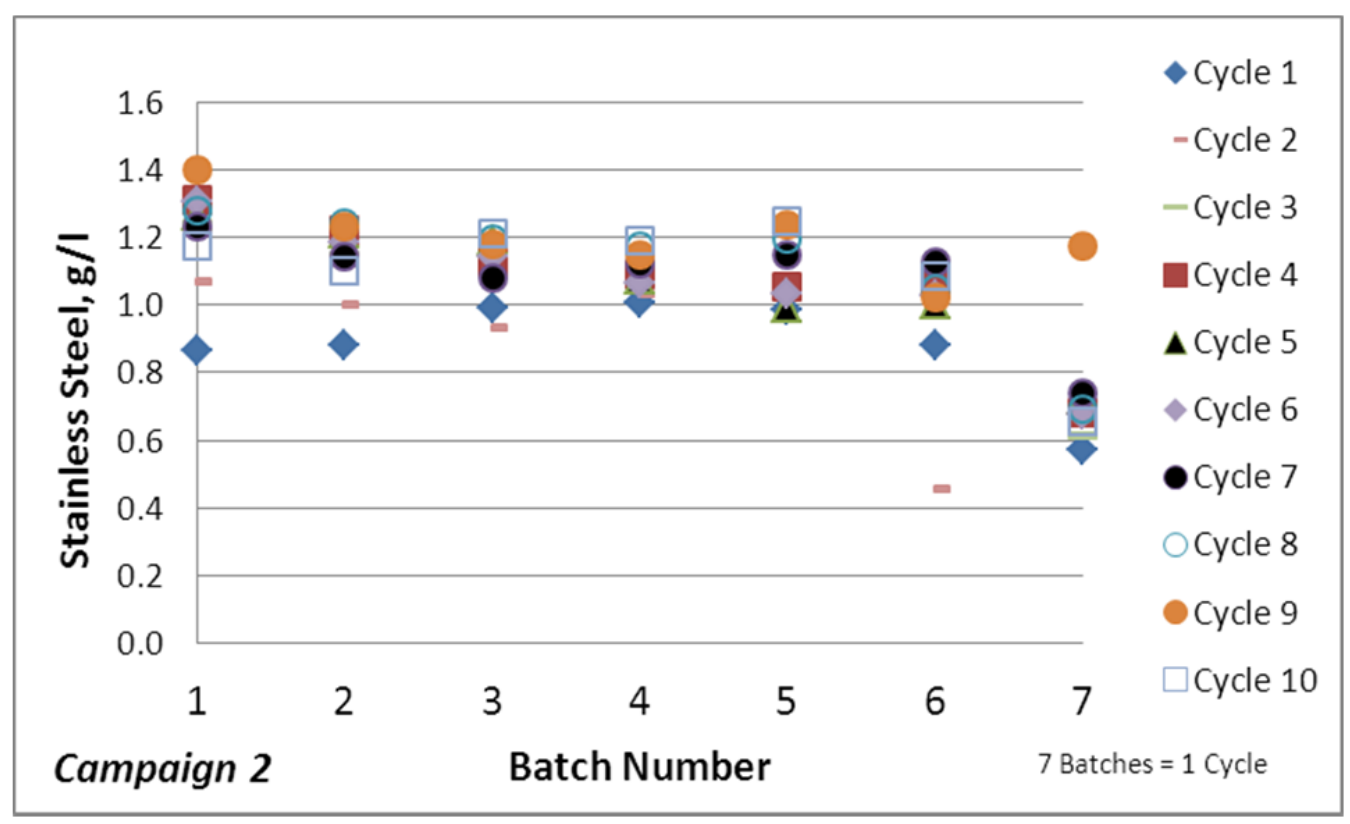

Figure 4-12. Batch to batch SS transferred during Campaign 2.

The cycle shown are expected to be a good representation of the transfer capacity of the system. Note, Batch 7 was the partial batch and therefore was smaller. 


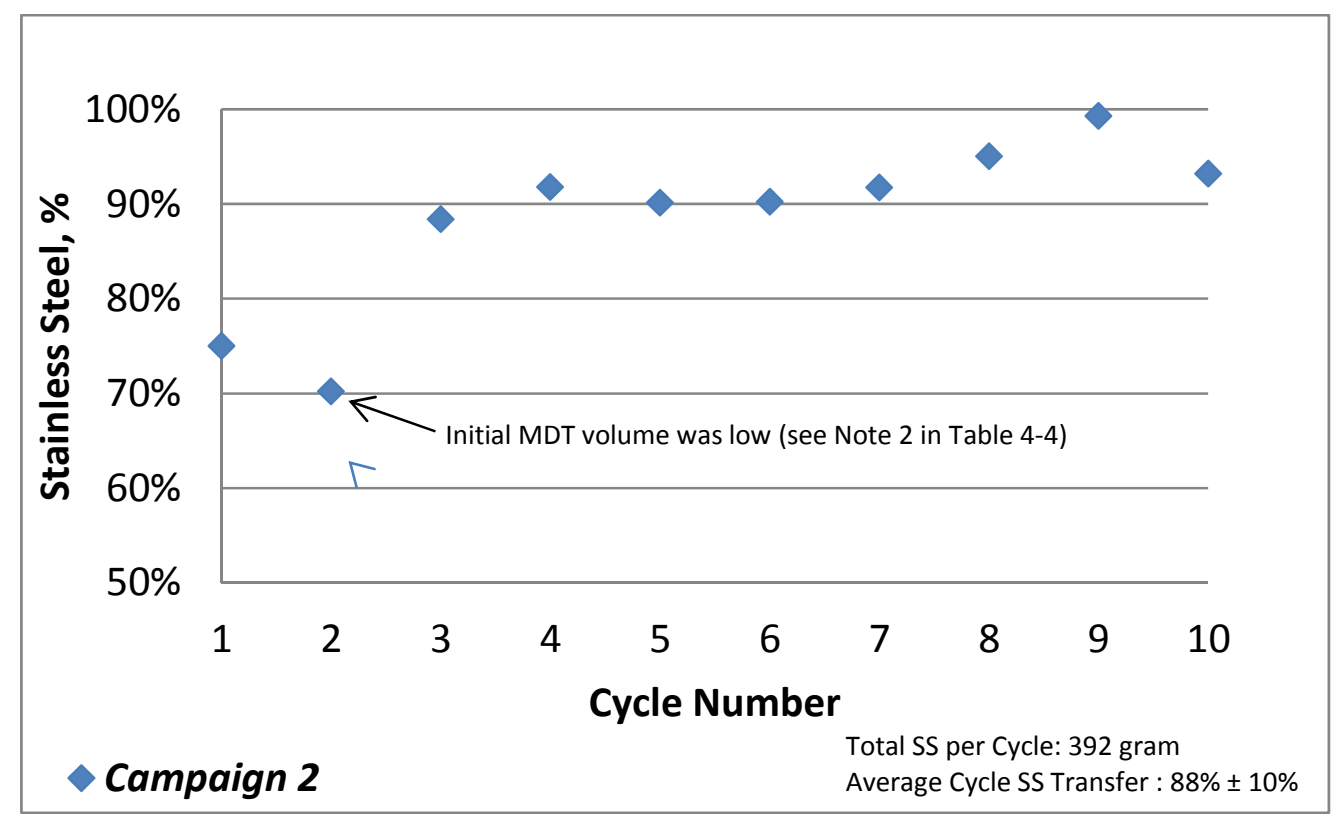

Figure 4-13. Shows the results of Figure 4-11 for each cycle.

That is, all seven batches per cycle were summed and normalized to the full cycle batch of SS available, i.e. 392 grams. Here it is clear that accumulation of the heavy SS particles does occur, but reaches a maximum at around the 7 th cycle.

\subsection{Solids Accumulation in the MDT}

The accumulated solids were measured several different ways: During batch-to-batch transfers the solids mounds in the MDT were photographed through the bottom of the transparent tank to roughly estimate the mound footprints. At the end of Cycles 1, 5, and 10 with only a heel of simulant remaining, when the mounds were measured for volume and core samples were taken from the mounds. Finally, at the end of each campaign when all of the accumulated solids were collected to estimate the total amount of stainless steel left behind after 10 complete fill and transfer cycles.

\subsubsection{Solids Accumulation - Photographic Evidence}

On each day of the test photographs were taken ${ }^{5}$ of the transparent bottom of the MDT to get a rough estimate of the life cycle of the accumulated solids from transfer to transfer. Because the simulant was opaque nothing, or very little, could be seen from the side or the top of the mixing simulant, but the mounds of accumulated solids could be observed from the bottom. Some examples are shown in

Figure 4-14(a)-(d).

\footnotetext{
${ }^{5}$ Starting with Campaign 2 videos were also taken of the MJP movement for each batch. The list is in Appendix D: List of Videos Taken.
} 
Revision 0

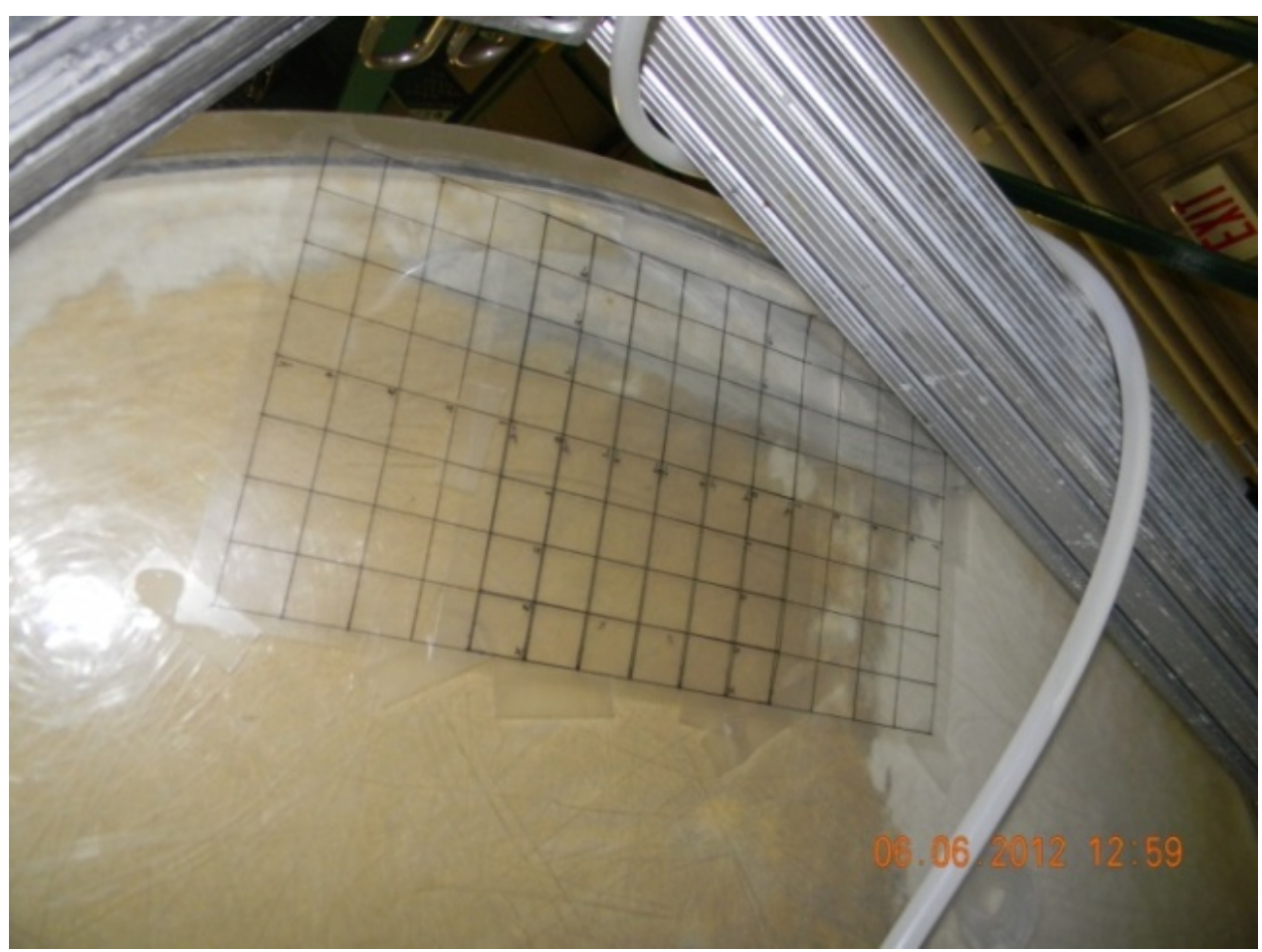

(a) Campaign 1 South Mound

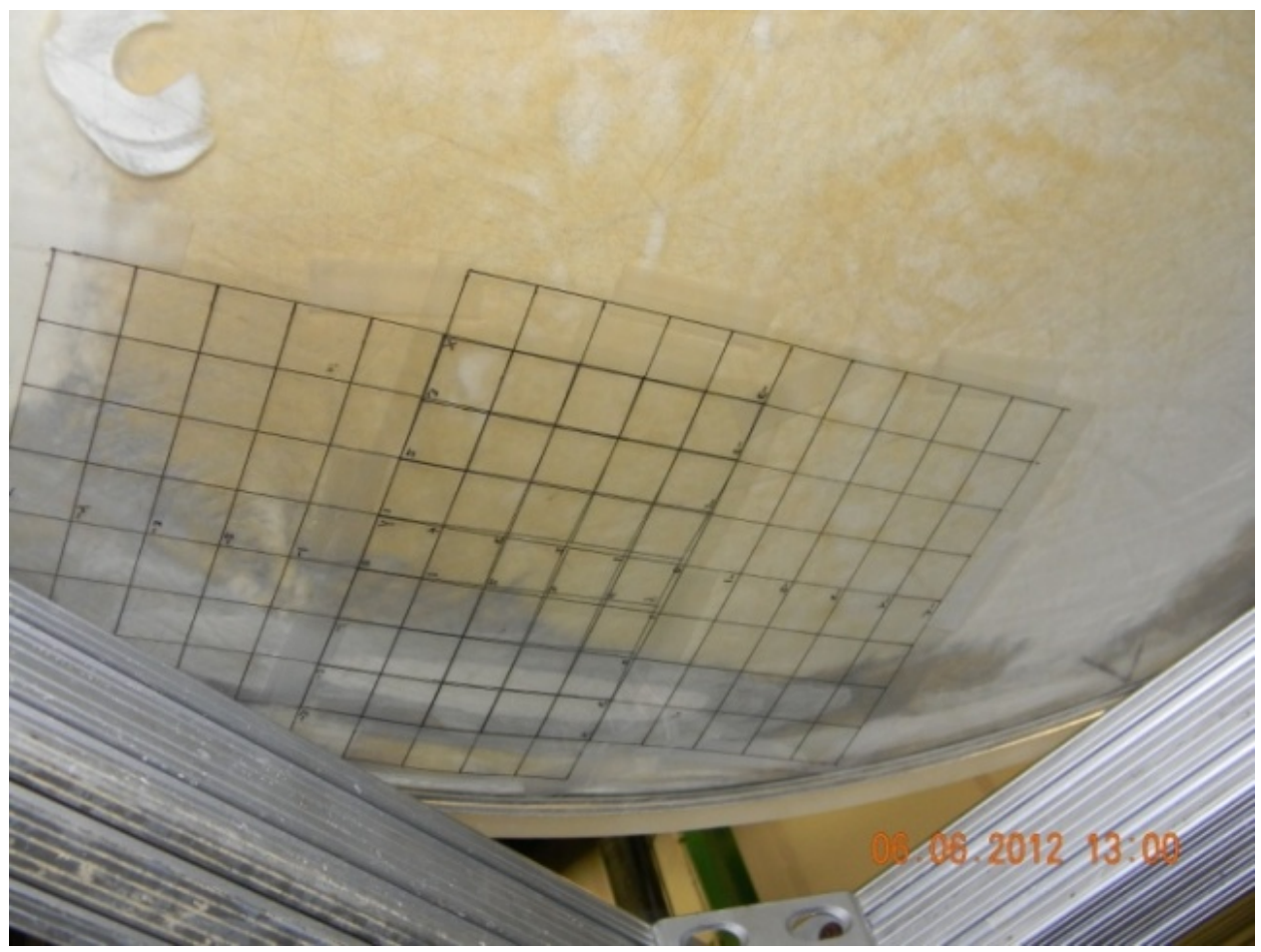

(b) Campaign 1 North Mound 
Revision 0

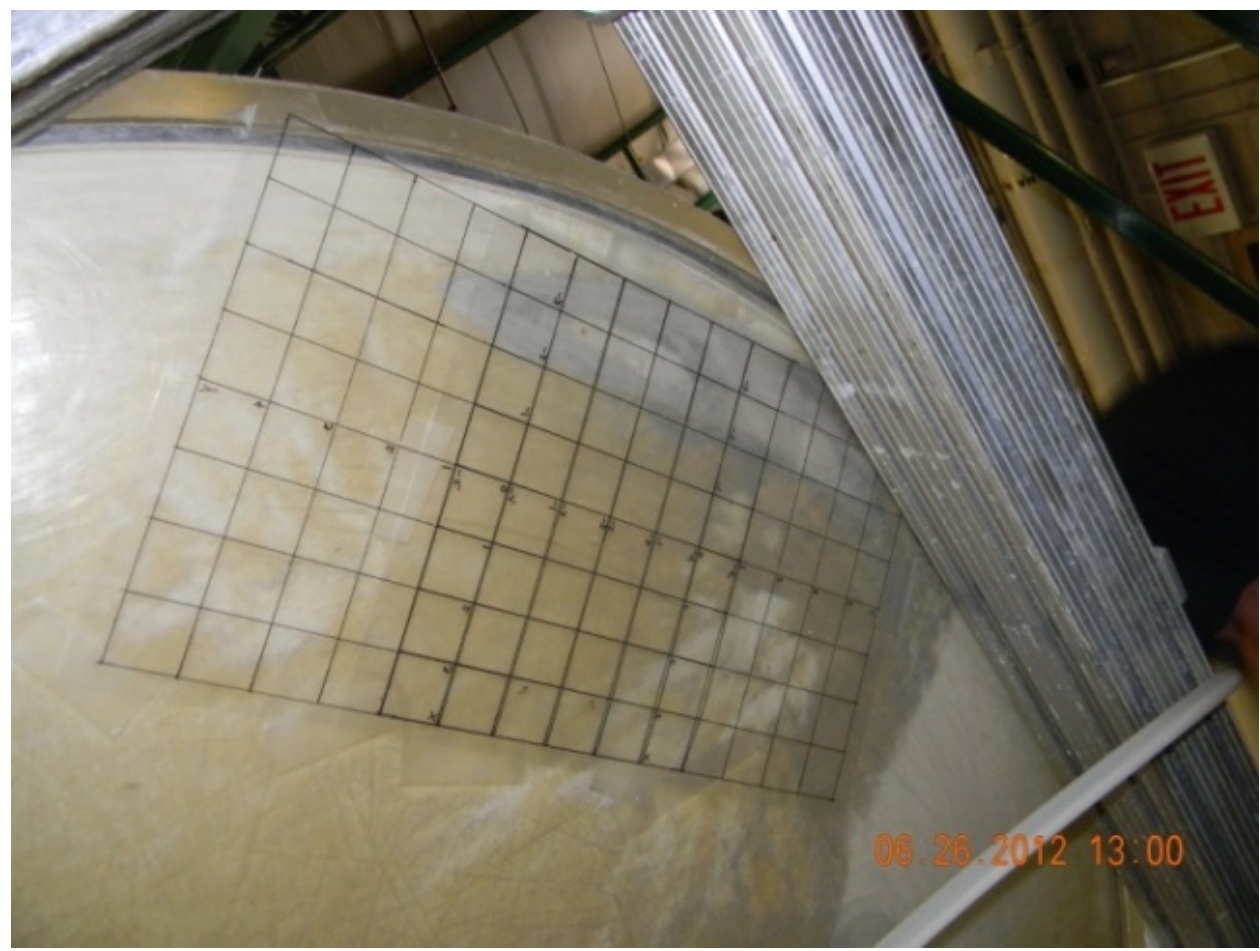

(c) Campaign 2 South Mound

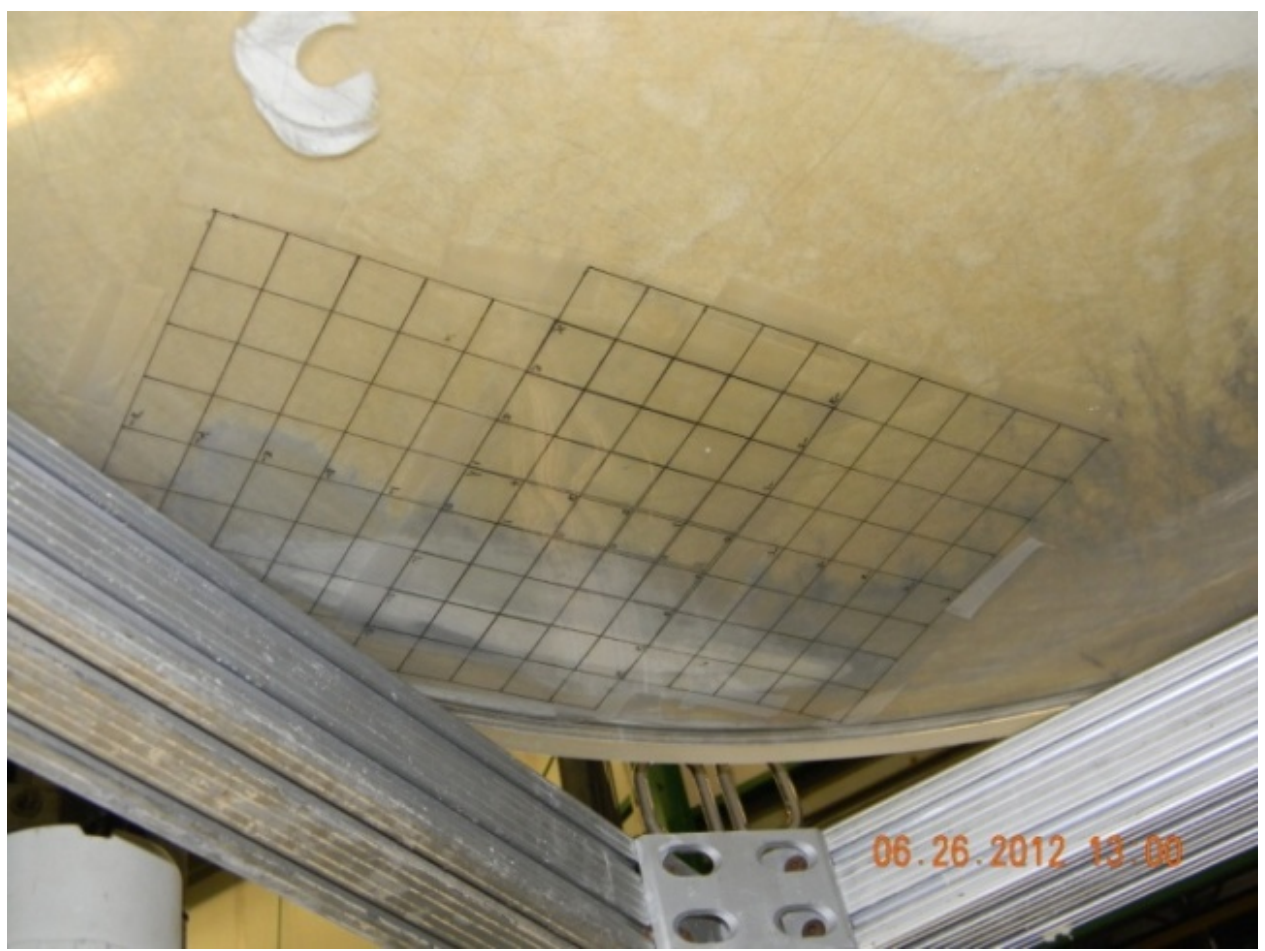

(d) Campaign 2 North Mound

Figure 4-14 (a) - (d). Accumulated solids footprints during Cycle 4 Batch Transfer. The grid used to measure size is in inches. 
In an attempt to quantify the mound changes, the mounds were roughly measured with the grids shown in

Figure 4-14 and the data are given in Figure 4-15, Figure 4-16, Figure 4-17, and Figure 4-18. However, in general the mound footprints were very uneven and hard to measure the length and width because of the very irregular shapes. That is, the measure really does not give a good quantitative estimate of mound size or shape, but it is useful to understand the qualitative movement of the accumulated solids.

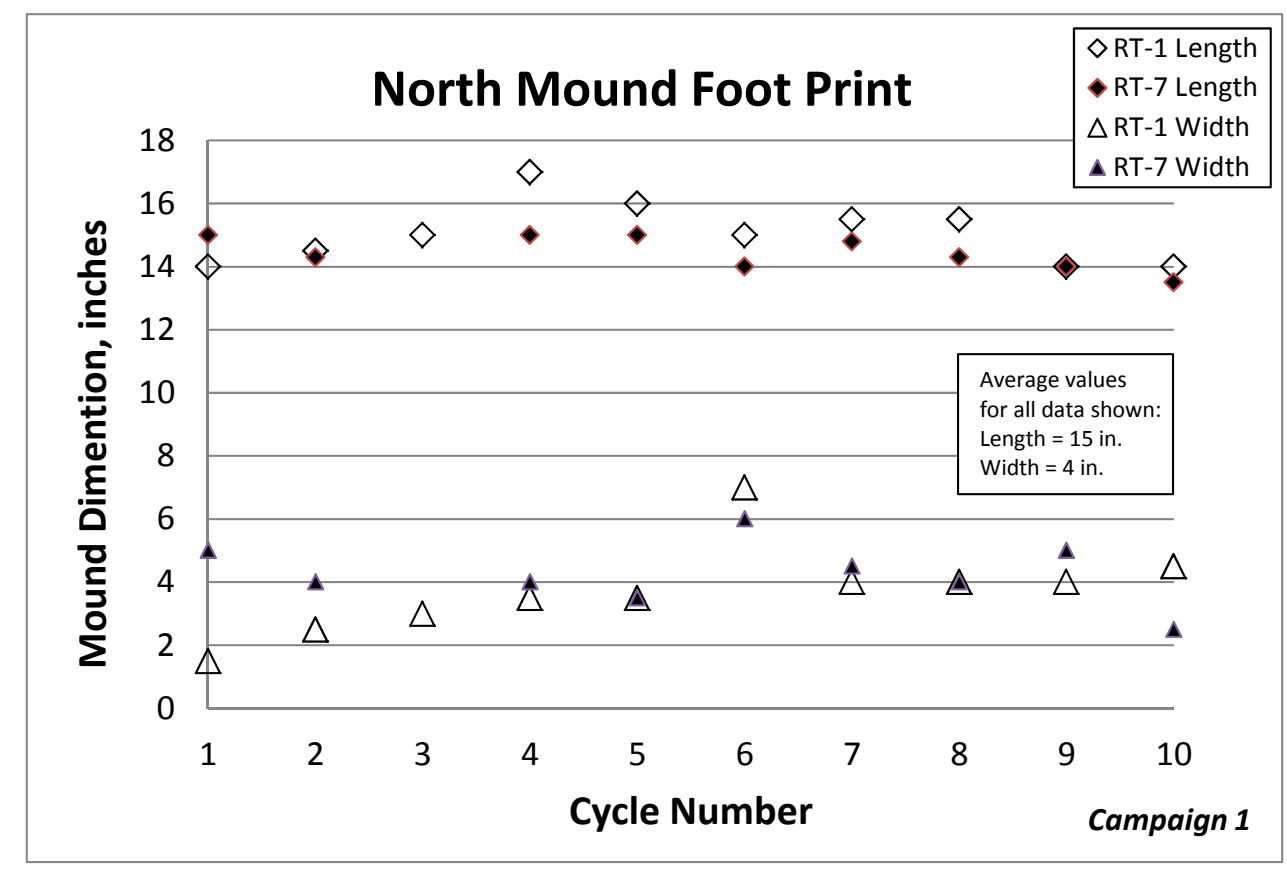

Figure 4-15. Campaign 1 cycle to cycle "north mound" accumulated solids foot print at the first and seventh transfer indicating the approximate length and largest width.

Overall the mounds did not move from their general locations. The two mounds were on opposite sides of the tank, referred to as the North and South mounds, and in the areas furthest from the mixing pumps. Roughly the footprints were crescent in shape, which grew and shrank slightly from batch to batch. The only significant difference happened during the last transfer when the mounds tended to spread out more because of the lower level of tank simulant. However, when the tank was refilled and the new cycle started the mounds generally returned to the original sizes.

It appears that with the slightly higher MJP velocity of Campaign 2, i.e., $23.9 \mathrm{ft} / \mathrm{s}$, Table 4-2, did have an effect because mound footprints were slightly smaller than in Campaign 1, which had an average velocity of $22.9 \mathrm{ft} / \mathrm{s}$, Table 4-1. The size of the North mound of Campaign 1 averaged about 15 inches long and 4 inches wide, Figure 4-15, while for Campaign 2 it averaged about 14 inches long and 3 inches wide, Figure 4-17. The South mound of Campaign 1 averaged about 12 inches long and 3 inches wide, Figure 4-16, while for Campaign 2 it averaged about 11 inches long and 2 inches wide, Figure 4-18. 


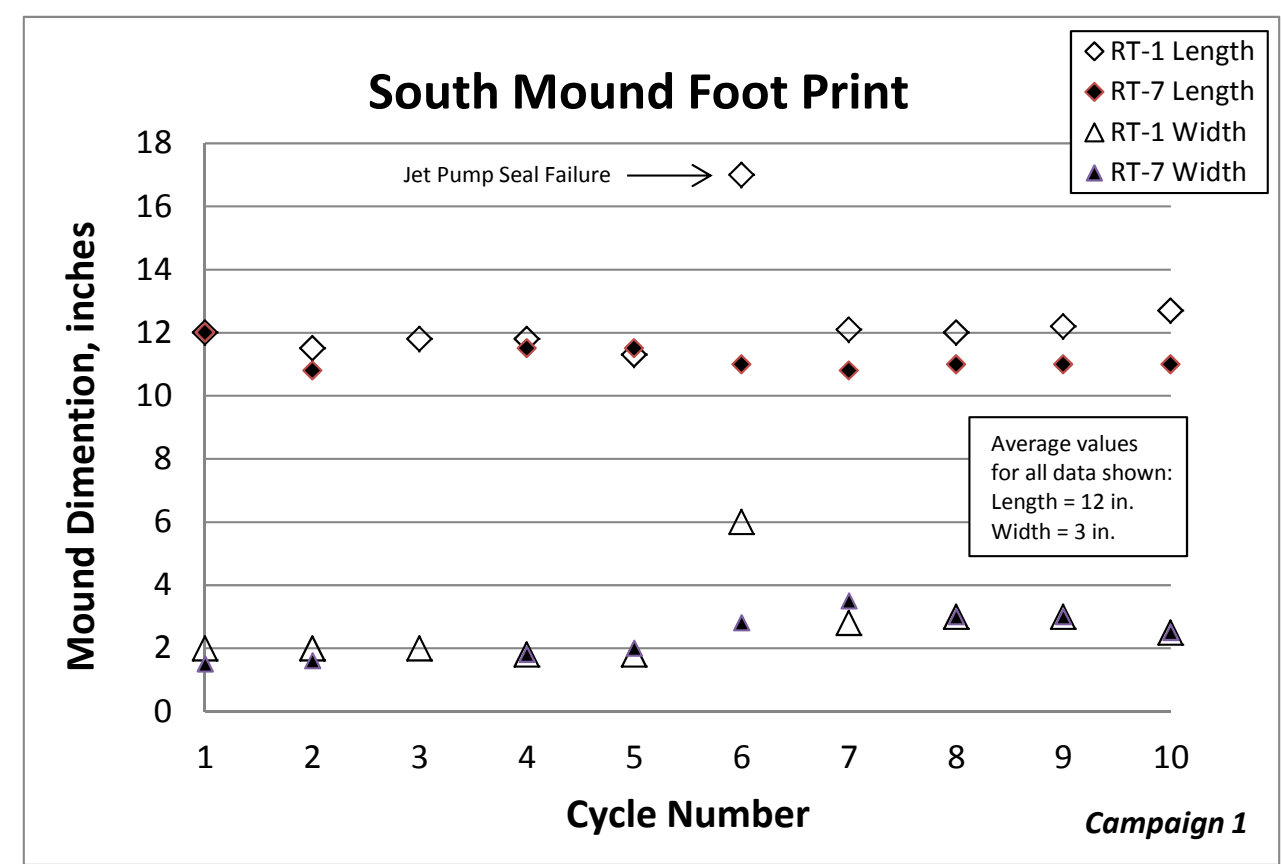

Figure 4-16. Campaign 1 cycle to cycle "south mound" accumulated solids foot print at the first and seventh transfer indicating the approximate length and largest width.

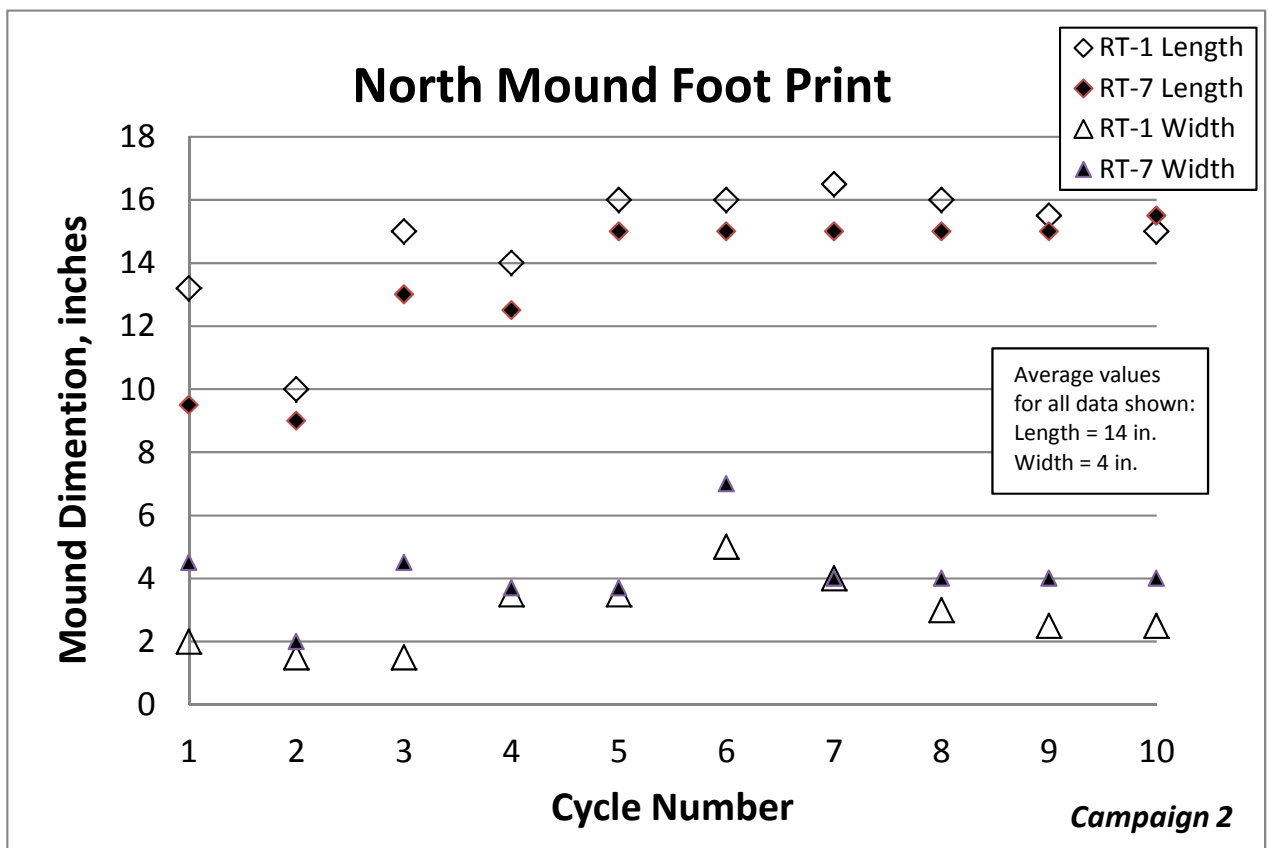

Figure 4-17. Campaign 2 cycle to cycle "north mound" accumulated solids foot print at the first and seventh transfer indicating the approximate length and largest width. 


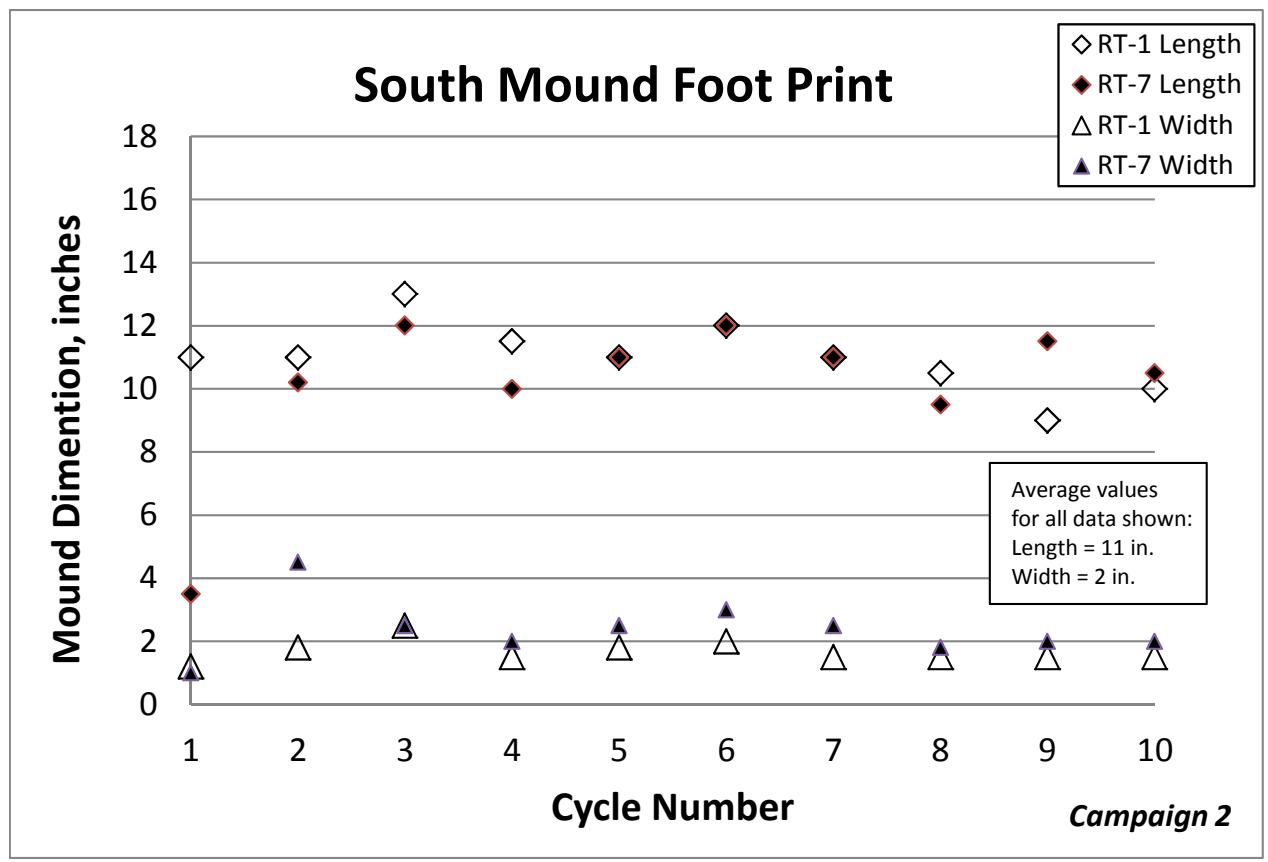

Figure 4-18. Campaign 2 cycle to cycle "south mound" accumulated solids foot print at the first and seventh transfer indicating the approximate length and largest width.

\subsubsection{Heel Measurements}

The amount stainless steel (SS) transferred from each fill and empty of a staging tank only indirectly gave information of what was left in a tank once the transfer was complete. To obtain a better measure of the solids accumulated in a tank the mounds needed to be measured directly; therefore, for SASS the residual solids were measured three ways:

1. Estimating the volumes of two mounds that formed in the poorly mixed sections of the tank after Cycles 1, 5 and 10 of both campaigns.

2. Taking samples from those mounds to determine spacial concentrations of the stainless steel particles after Cycle 1, 5 and 10 of both campaigns. Only one mound was sampled after Cycles 1 and 5, but both mounds were sampled after Cycle 10.

3. Removing all the stainless steel particles left in bottom of the MDT at the end of each campaign to measure the residual mass.

Three times during each campaign these direct, heel, measurements, were made, specifically after the $1^{\text {st }}, 5^{\text {th }}$, and $10^{\text {th }}$ cycles. During all heel measurements most of the heel material was gently drained to expose, but not disturb, the accumulated solids mounds. Those mounds were then scanned with lasers to determine their volumes and shapes, sampled with the core sampler, and finally, as the heel material was returned to the MDT, the mounds were photographed at fixed and known liquid levels, as another method to estimate the volumes of the mounds. However, of the three heel measurement events only after the $10^{\text {th }}$ cycle of each campaign were core samples taken from the South mound. The rationale was to only disturb one of the two mounds after the $1^{\text {st }}$ and $5^{\text {th }}$ cycle so that after the $10^{\text {th }}$ cycle there would be one mound undisturbed and thereby increase the chances of more accurate measurements and compare that mound's results to the disturbed mound. As will be discussed later, the core sampling disturbed the mounds 
insignificantly because the total mass removed was only $2 \%$ to $3 \%$ of the mass of the mound. Furthermore, the North and South mounds were of significantly difference sizes, which made a one to one comparison impossible.

\subsubsection{Determining Volume of the Accumulated Solids}

The volume of the mounds of accumulated solids were estimated two ways: Measuring the shape of the mounds using a laser and photographing exposed sections of the mounds at known levels of simulant to estimate the exposed mound surfaces to then integrate all the area versus height estimates to obtain a volume. The results are described below along with a final section to compare the volume estimates from both methods.

\subsection{Volume Estimation from Laser Measurements}

With the equipment shown in Figure 3-6, Figure 3-7, and Figure 3-8 laser measurements were made to map the bottom of the MDT to obtain 3-D information of the accumulated solids mounds. Sections 3.7 and 3.8 explained the equipment, its operation; and how the results were obtained. Those volumetric results obtained from the laser scans are shown in Figure 4-19 and the mounds are displayed both in graphical and photographical evidence the following 36 figures.

The laser scan graphs are in chronological order to see the development of the mounds of solids that accumulated with time. To better understand the actual size of the mounds for each cycle is a photograph of the North and South mounds. To actually measure the mounds the scale of the laser data are expanded, which make the mounds look much larger that they were. Furthermore, for each mound in each cycle there are two laser measurement views: First, a side view, which has the perspective of looking at the mound from the center of the tank, and second, a top view of same mound.

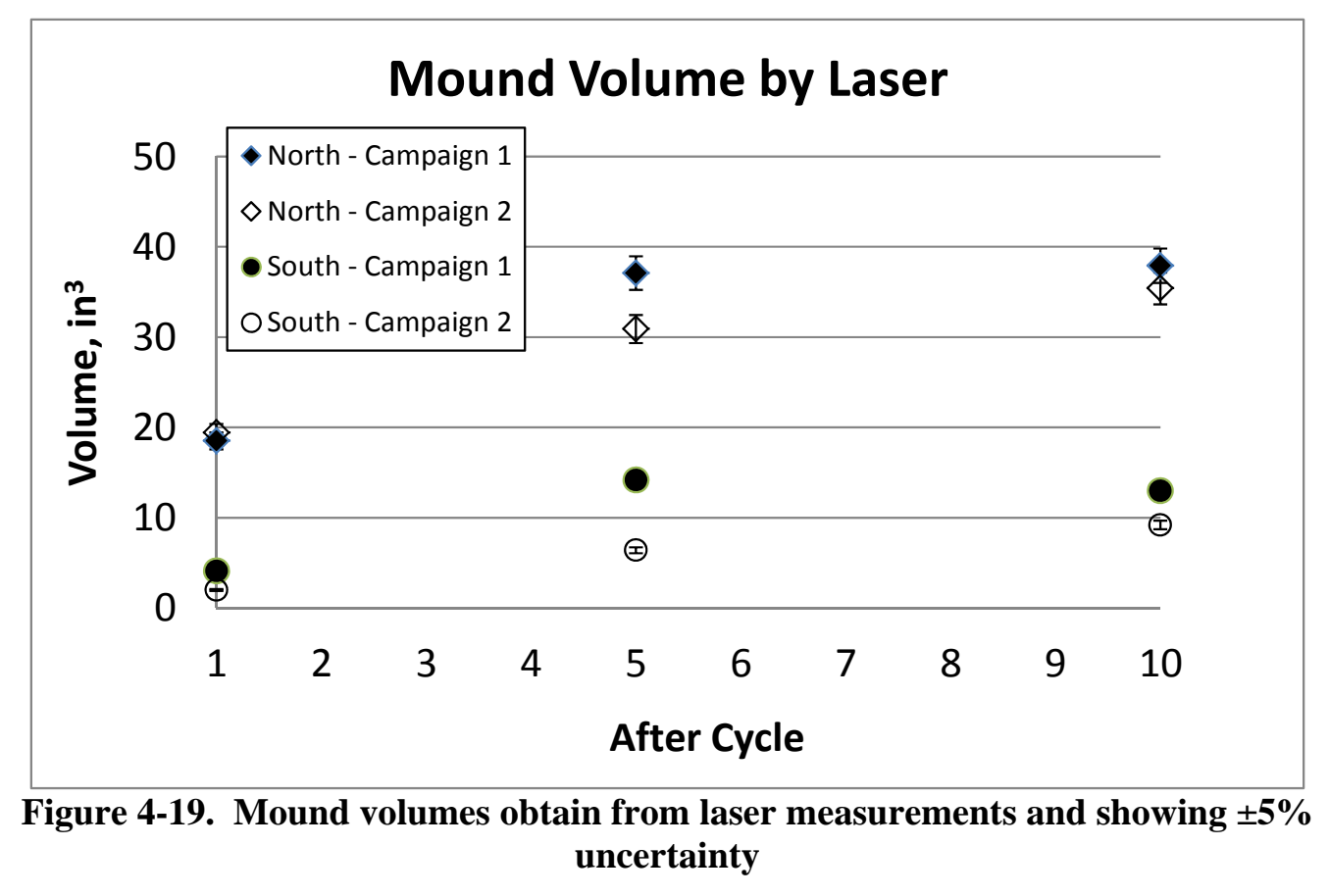


Photographs Campaign 1:

-, Figure 4-20 \& Figure 4-21, Figure 4-26 \& Figure 4-27, Figure 4-32 \& Figure 4-33

Photographs Campaign 2

- Figure 4-38 \& Figure 4-39, Figure 4-44 \& Figure 4-45, Figure 4-50 \& Figure 4-51

There was no systematic method of taking photographs for the laser measurement; therefore, the figures listed above were of pictures taken either to highlight interesting details or during one of the other measurement methods, e.g., core sampling or determining volume with photographs. Furthermore, all of the pictures were taken from above and orientation was not considered. However, to compare them to the laser graphs of the mounds note that the circular surface in all the photographs is MDT wall and therefore the outer edge of the mounds. The average diameter of the MDT was 40.5 inches so the radial dimension towards the wall was 0 inches at the center and 20.25 inches at the wall. The radial dimension is shown on all the laser graphs. The following are some facts about the photographs:

Figure 4-20 and Figure 4-21 show a ruler with a dimension and two machine bolts, which was the initial method used to show scale. The bolts were used to superimpose pictures so scaling would be accurate. These pictures were taken during the photographic volume determination phase of the heel measurements with Figure 4-20 showing the tank level at 0.2 inches and Figure 4-21 at 0.4 inches.

Figure 4-26 and Figure 4-27 illustrate show why it was important to empty the MDT of the heel material and the small gibbsite and zirconia particles. Figure 4-26 shows that most of the North mound was successfully exposed for measurement; however, Figure 4-27 shows the very small South mound completely covered with a thin layer of the small gibbsite and zirconia particles which challenged the photographic method to determine volume because of the difficulty of distinguishing the liquid-solid interface while the tank level was slowly raised.

Figure 4-32 and Figure 4-33 show the mounds after the core samples were collected and just before the photographic volume determining technique was to begin. Starting with Cycle 10 of Campaign 1 the method of determining scale and showing tank level was changed. The ruler and tape, shown in Figure 4-32 and Figure 4-33 were replaced by a plastic arrowhead-shaped platform. The platform served four purposes:

1. It stood on three nails with the upper surface being exactly 3 inches and therefore was used to adjust the laser measurements to a known height.

2. It contained a manually adjustable indicator to register the tank level from photographs that ranged from 0.01 inch to 2.5 inches.

3. It contained either the letter $\mathrm{N}$ or $\mathrm{S}$ to indicate the North or South mounds, respectively.

4. Like the machine bolts in Figure 4-32 and Figure 4-33, the dimensions of the platform were used to superimposed photographs so they were scaled properly to accurately estimate a mound volume.

As previously discussed the North mound was partially exposed after the heel level was lowered but the South mound was completely covered.

Figure 4-38, Figure 4-39, Figure 4-44, Figure 4-45, Figure 4-50, and Figure 4-51 show the photographs taken during Campaign 2, immediately after the core samples were taken and in preparation for the photographic volume determination technique. Note that after Cycle 1, Figure 
4-39, and after Cycle 5, Figure 4-45, core sample were not taken from the South mound, but they were taken from the South mound after Cycle 10, Figure 4-51. Also, in Figure 4-39 the top left corner of the picture shows a 3-inch tall aluminum block that was placed in the tank for calibration check purposes, which was already discussed in Section 3.8.1 and the results were shown in Table 3-3.

Laser Graphs Campaign 1

- Figure 4-22 to Figure 4-25, Figure 4-28 to Figure 4-31, and Figure 4-34 to Figure 4-37

\section{Laser Graphs Campaign 2}

- Figure 4-40 to Figure 4-43, Figure 4-46 to Figure 4-49, and Figure 4-52 to Figure 4-55

As can be seen on the following laser graphs the locations of the mounds was on either side of the MDT and referred to as the North and South Mounds. The extent of rotation of the laser measurements was $110^{\circ}$ and for the North side they ranged from $47^{\circ}$ to $157^{\circ}$ with respect to the pump axis and for the South side from $227^{\circ}$ to $337^{\circ}$. To show more detail on some of the graphs the range of angles plotted was reduced to $75^{\circ}$ from $52^{\circ}$ to $132^{\circ}$ on the north side and from $237^{\circ}$ to $312^{\circ}$ on the south side. Note that in the MDT the diameter that ran from $0^{\circ}-180^{\circ}$ along the centerline of the MJP as shown in Figure 2-7. The tank radius was approximately 20 inches therefore the span of the radial positions was from 10 to 20 inches, which encompassed the entire mounds. For mound heights there was no fixed upper range but the interval was either 0.2 inch or, for most of the graphs 0.1 inch, to show the detail because all of the mounds were under 1 inch. The topographical maps were color coded.

The three dimensional graphs of the mounds look much rougher than the photographs indicate and the many pointed peaks in the blue shaded areas, near the bottom of the tank, are an artifact of the resolution of the laser rangefinders, 0.04 inches. Furthermore, most of those peaks were very small, that is, they are on the order of 0.1 inch and within the measurement uncertainty for a laser height, which is $\pm 1.5 \mathrm{~mm}$ (0.06 inch), see Table 2-4.

Each of the figures includes the volume obtained from the laser measurements, which will be discussed later. Some of the important conclusions from the figures are:

1. The North Mound was 3 to 4 times larger than the South mound. This will be discussed further in a later section, but it was because MJP2 mixing velocity was slightly stronger than MJP1, i.e., about $1 \mathrm{ft} / \mathrm{s}$, see Table 4-1 and Table 4-2.

2. Sometime between Cycles 1 and 5 the mounds stopped growing and stabilized at a fixed volume. The sand transfer data, Figure 4-6 and Figure 4-7 imply the transfer concentration stabilized between Cycle 2 and 3. The stainless steel data from Campaign 2 , Figure 4-13, imply the transfer concentration stabilized at approximately Cycle 3 . The Campaign 1 data are slightly harder to predict, Figure 4-10, but the trend indicates this stabilization may have occurred at approximately Cycle 4. However, all the data appear to indicate that by Cycle 5 an asymptote was reached.

3. Campaign 1, the filled symbols, resulted in slightly larger North and South mounds implying that the slightly higher MJP velocity for Campaign 2, i.e., $22.9 \mathrm{ft} / \mathrm{s}$ to $23.9 \mathrm{ft} / \mathrm{s}$, respectively, suspended the solids and mixed the MDT slightly better, which allowed more solids to be transferred. 


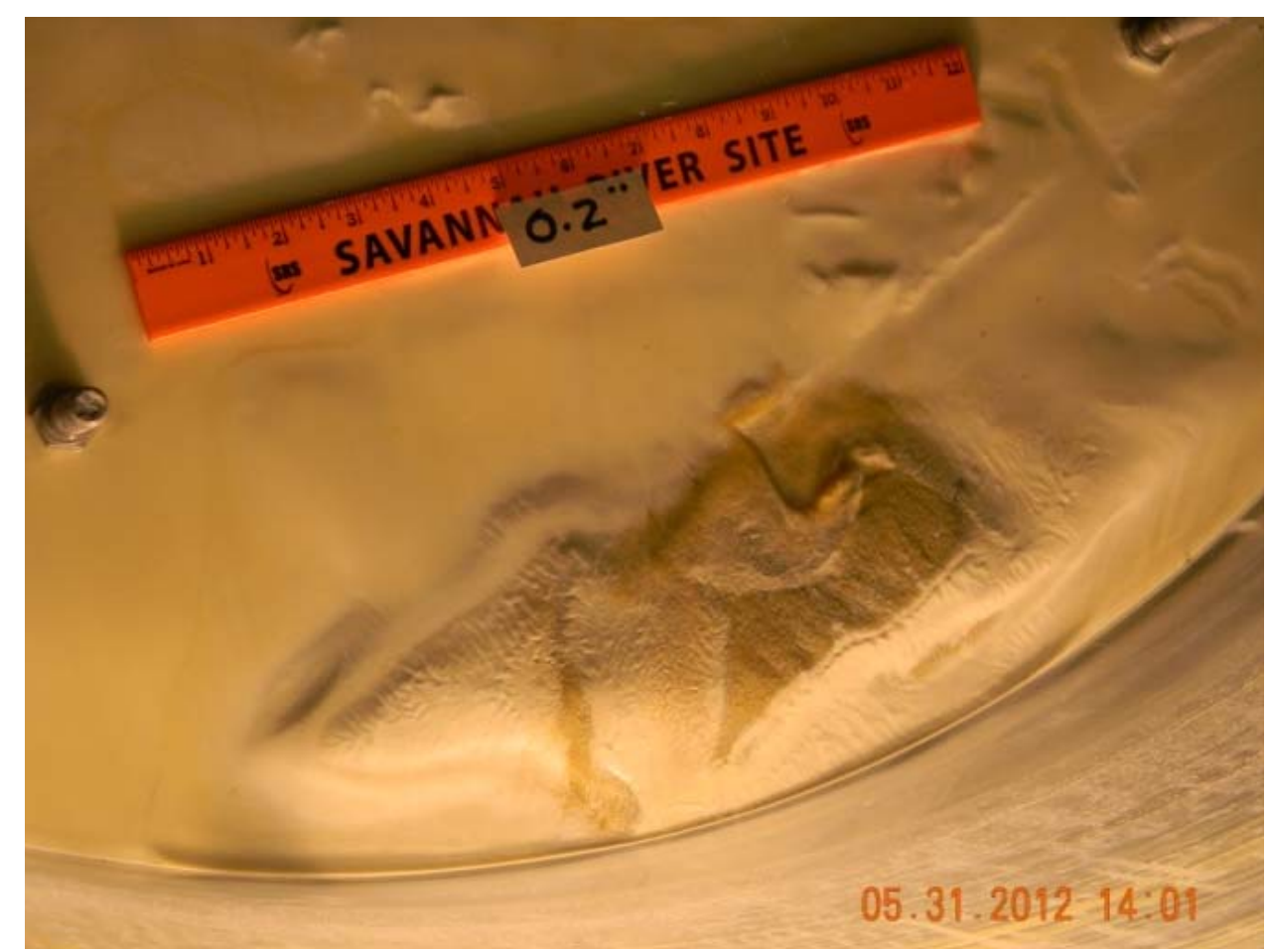

Figure 4-20. Solids North mound after Cycle 1 of Campaign 1.

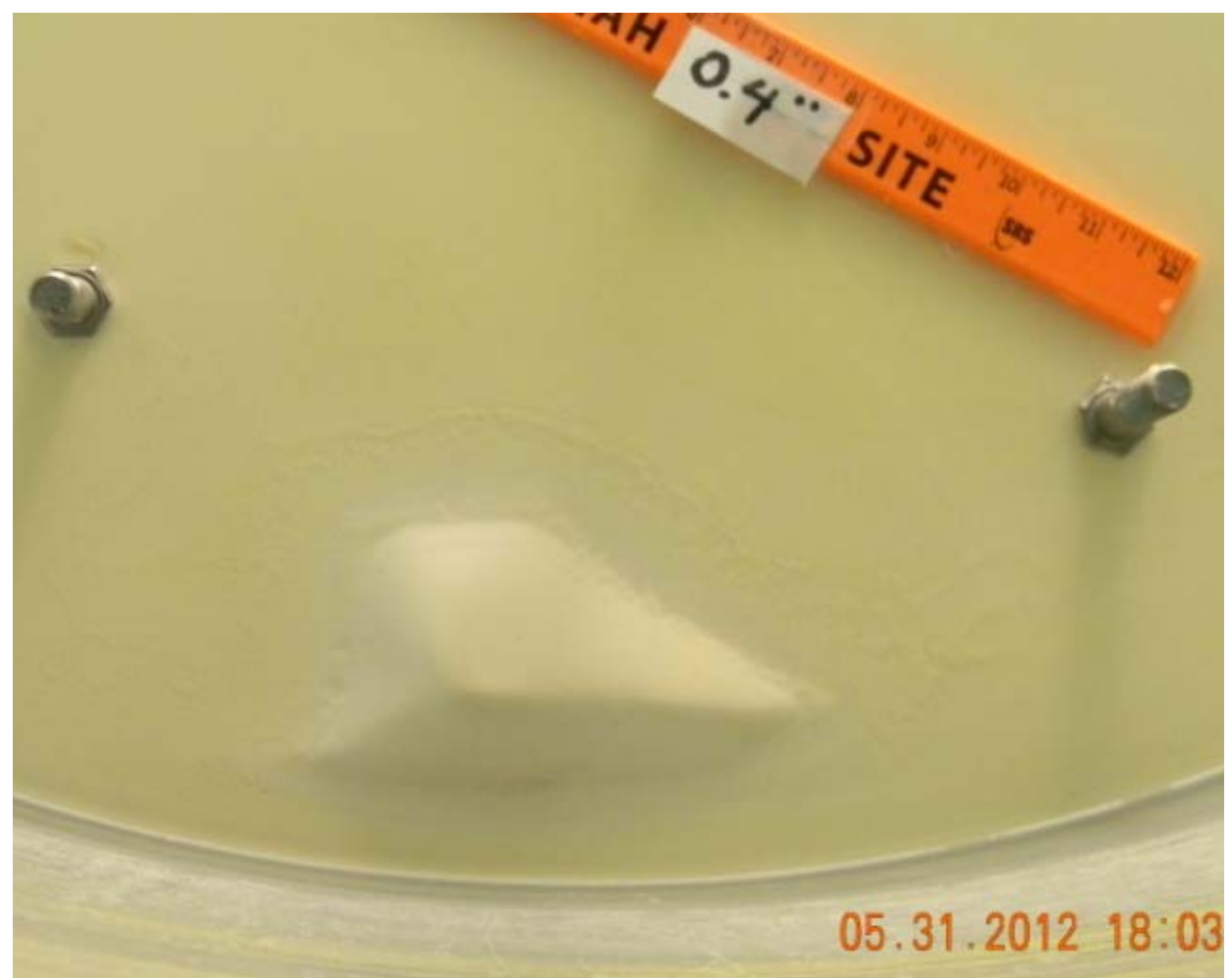

Figure 4-21. Solids South mound after Cycle 1 of Campaign 1. 


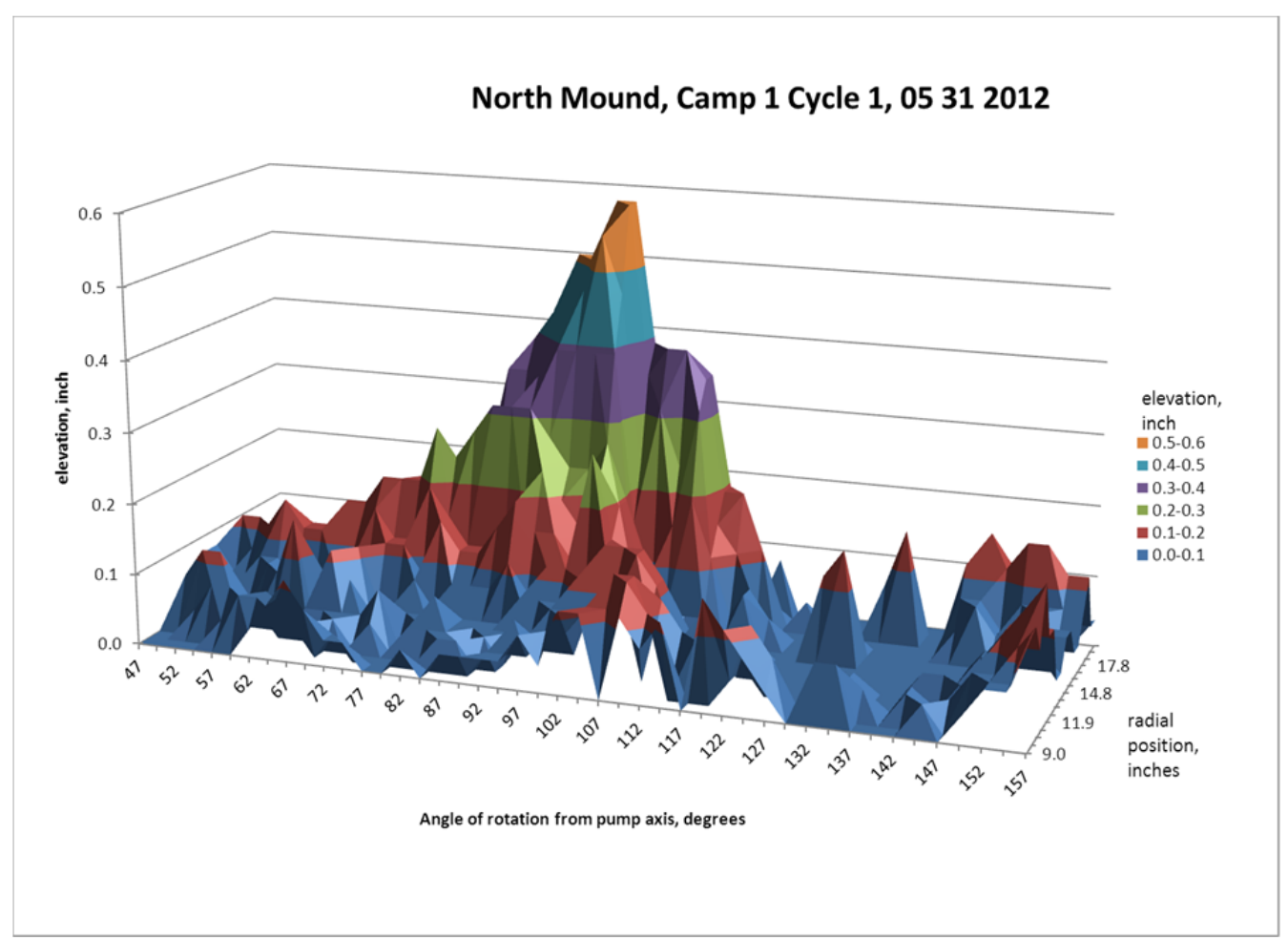

Figure 4-22. North mound side view after Cycle 1 of Campaign 1 - Volume of $18.5 \mathrm{in}^{3}$.

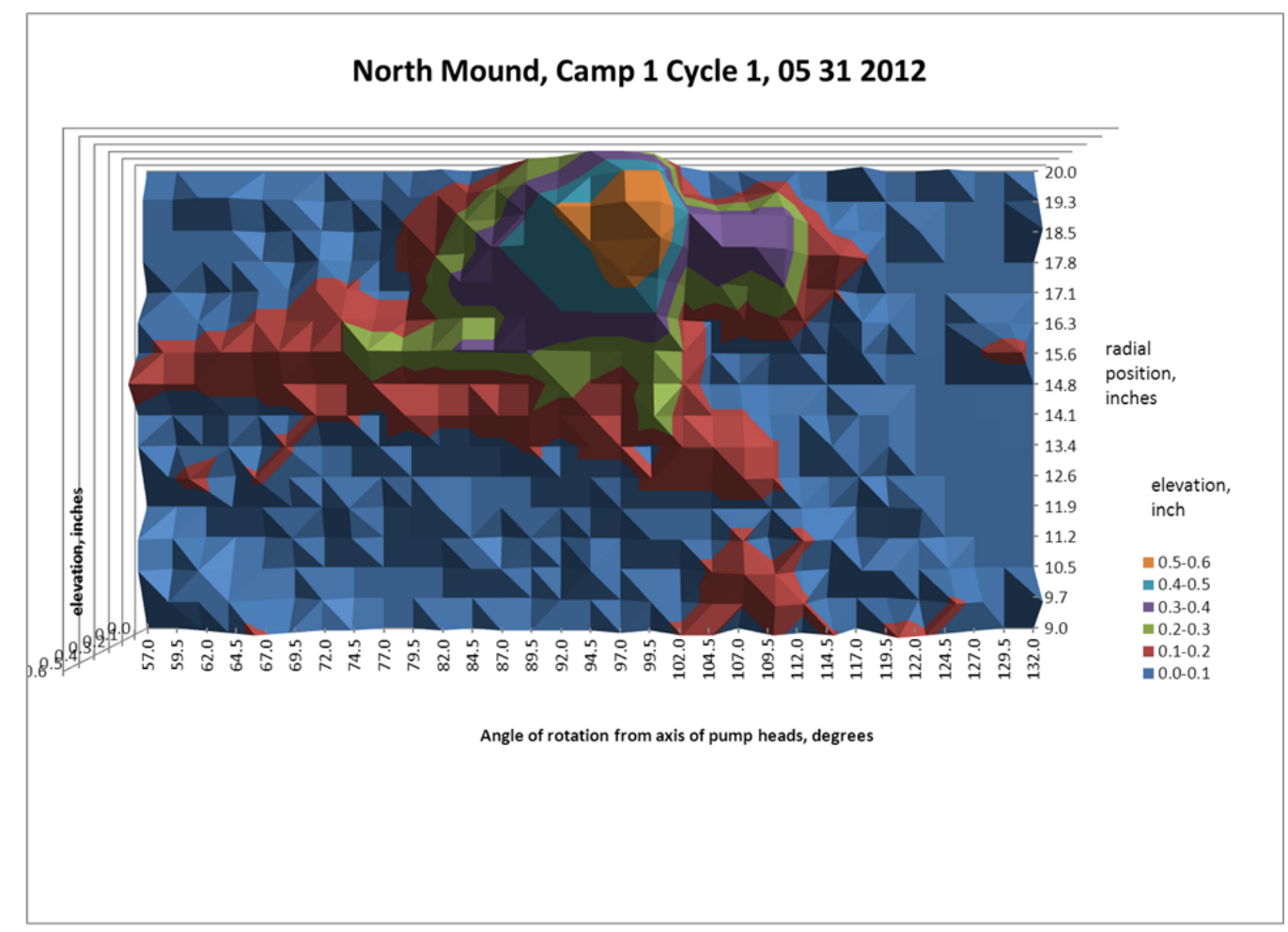

Figure 4-23. North mound top view after Cycle 1 of Campaign 1 - Volume of $18.5 \mathrm{in}^{3}$. 


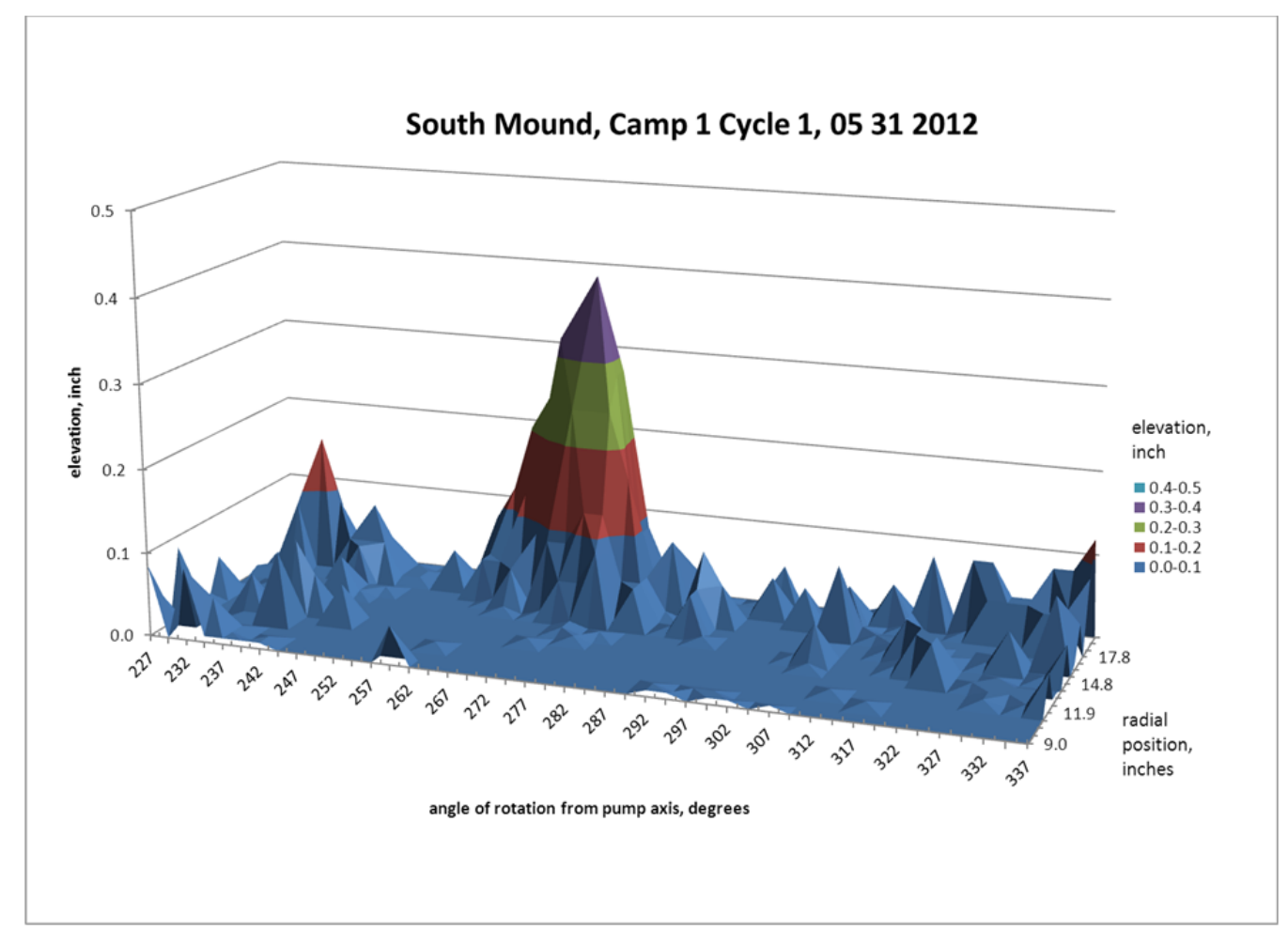

Figure 4-24. South mound side view after Cycle 1 of Campaign 1 - Volume of $4.1 \mathrm{in}^{3}$.

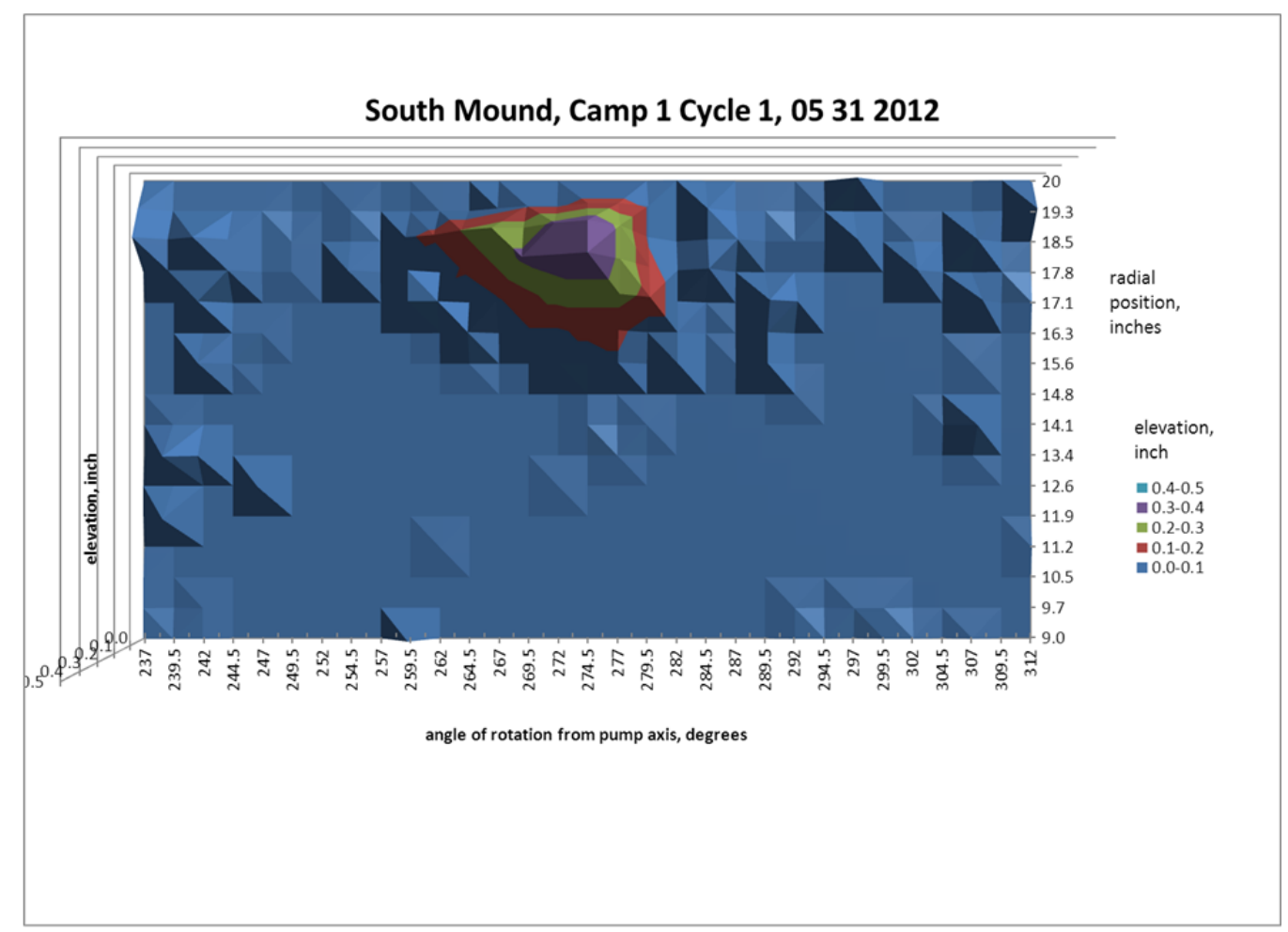

Figure 4-25. South mound top view after Cycle 1 of Campaign 1 - Volume of 4.1 in $^{3}$. 
Revision 0

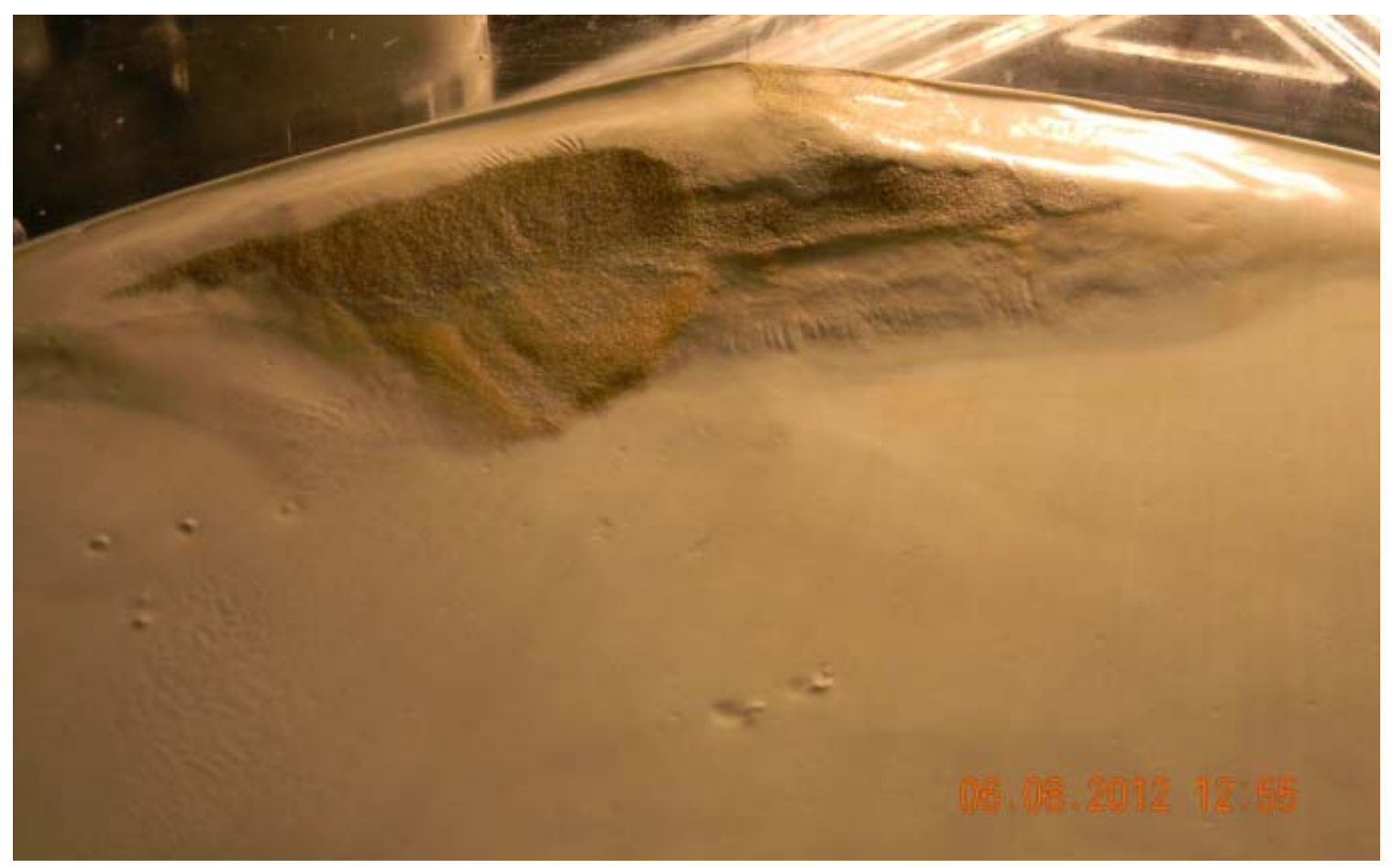

Figure 4-26. Solids North mound after Cycle 5 of Campaign 1.

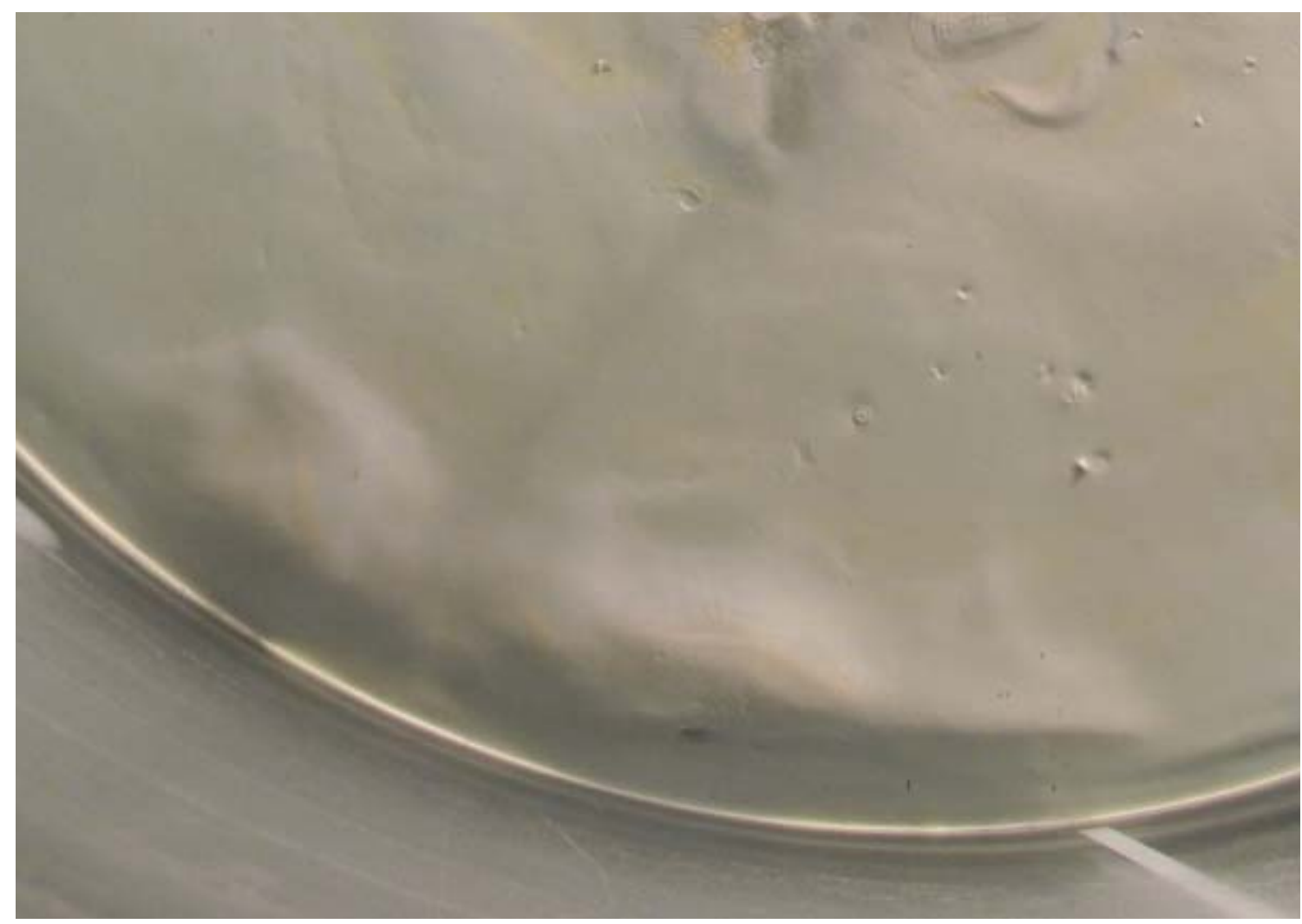

Figure 4-27. Solids South mound after Cycle 5 of Campaign 1. 


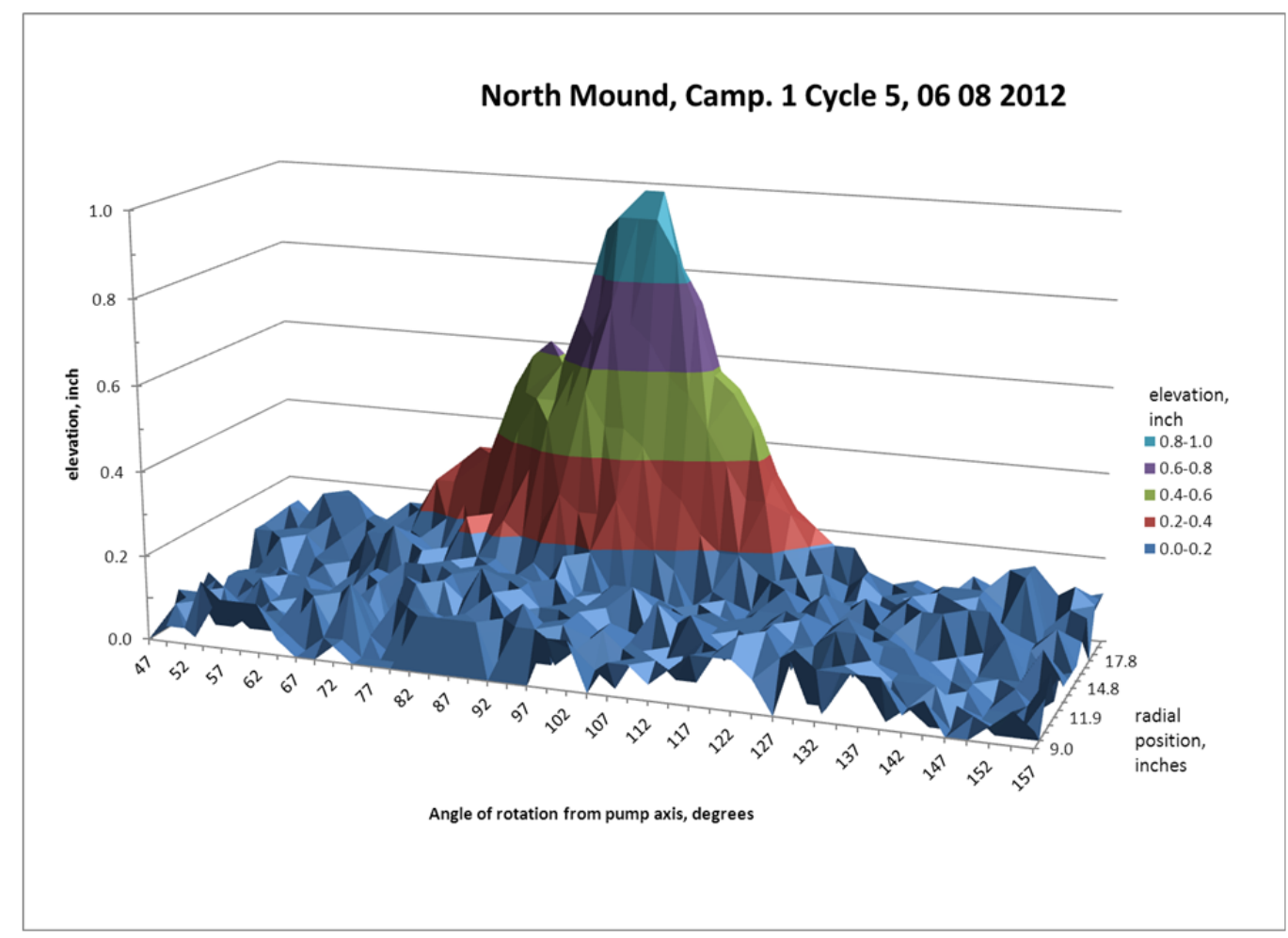

Figure 4-28. North mound side view after Cycle 5 of Campaign 1 - Volume of 37.1 in $^{3}$.

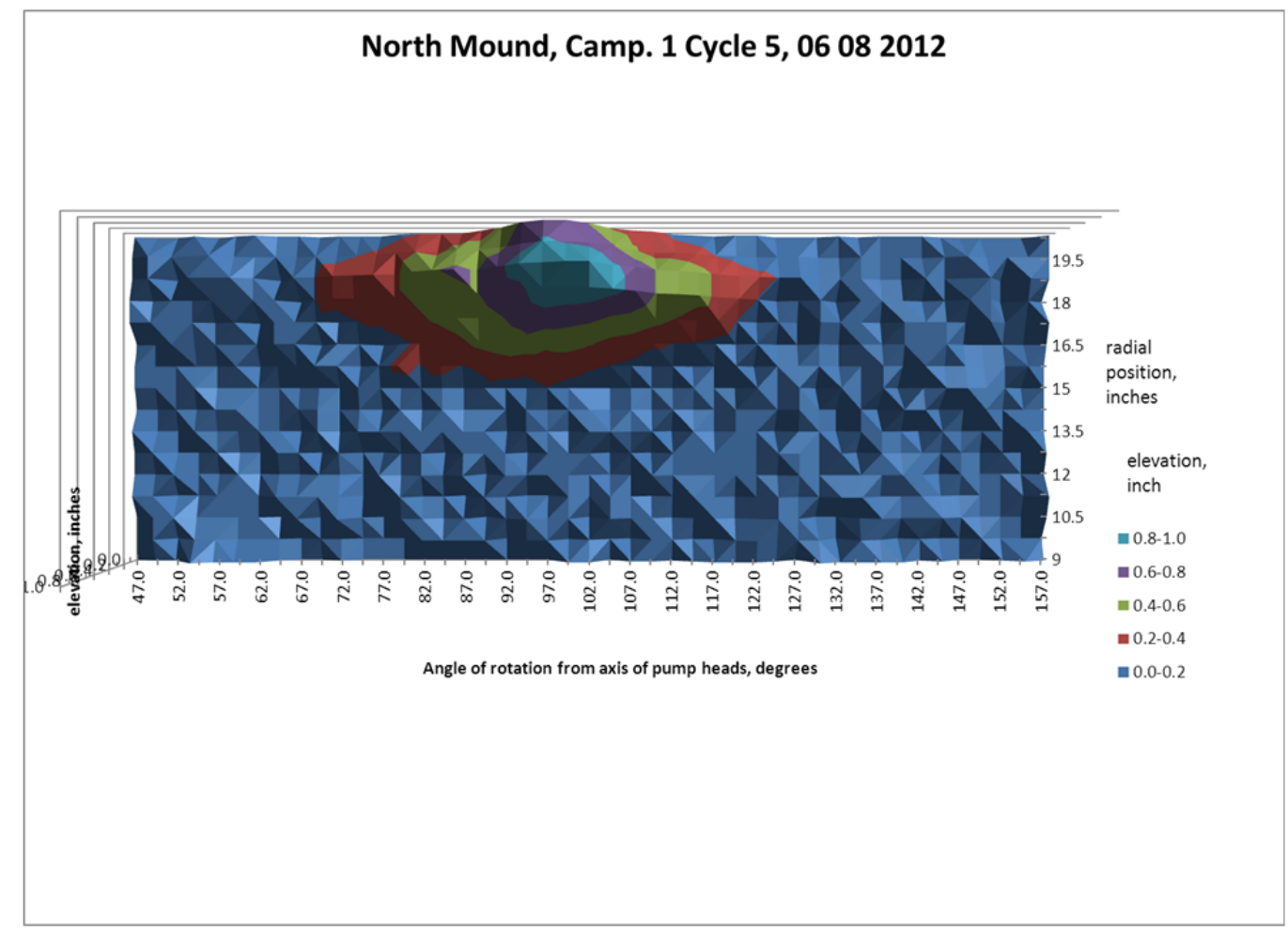

Figure 4-29. North mound top view after Cycle 5 of Campaign 1 - Volume of $37.1 \mathrm{in}^{3}$. 


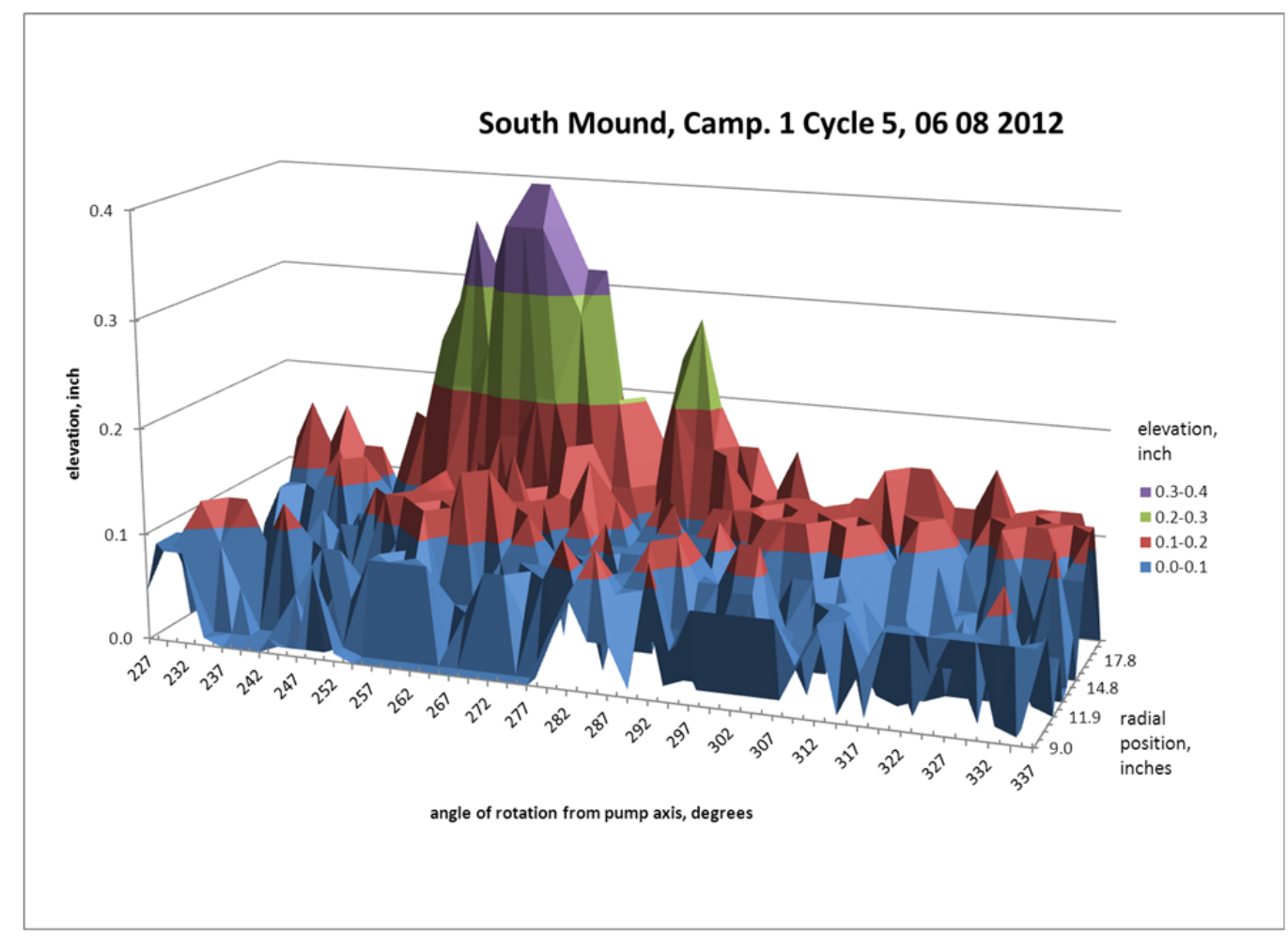

Figure 4-30. South mound side view after Cycle 5 of Campaign 1 - Volume of $14.2 \mathrm{in}^{3}$.

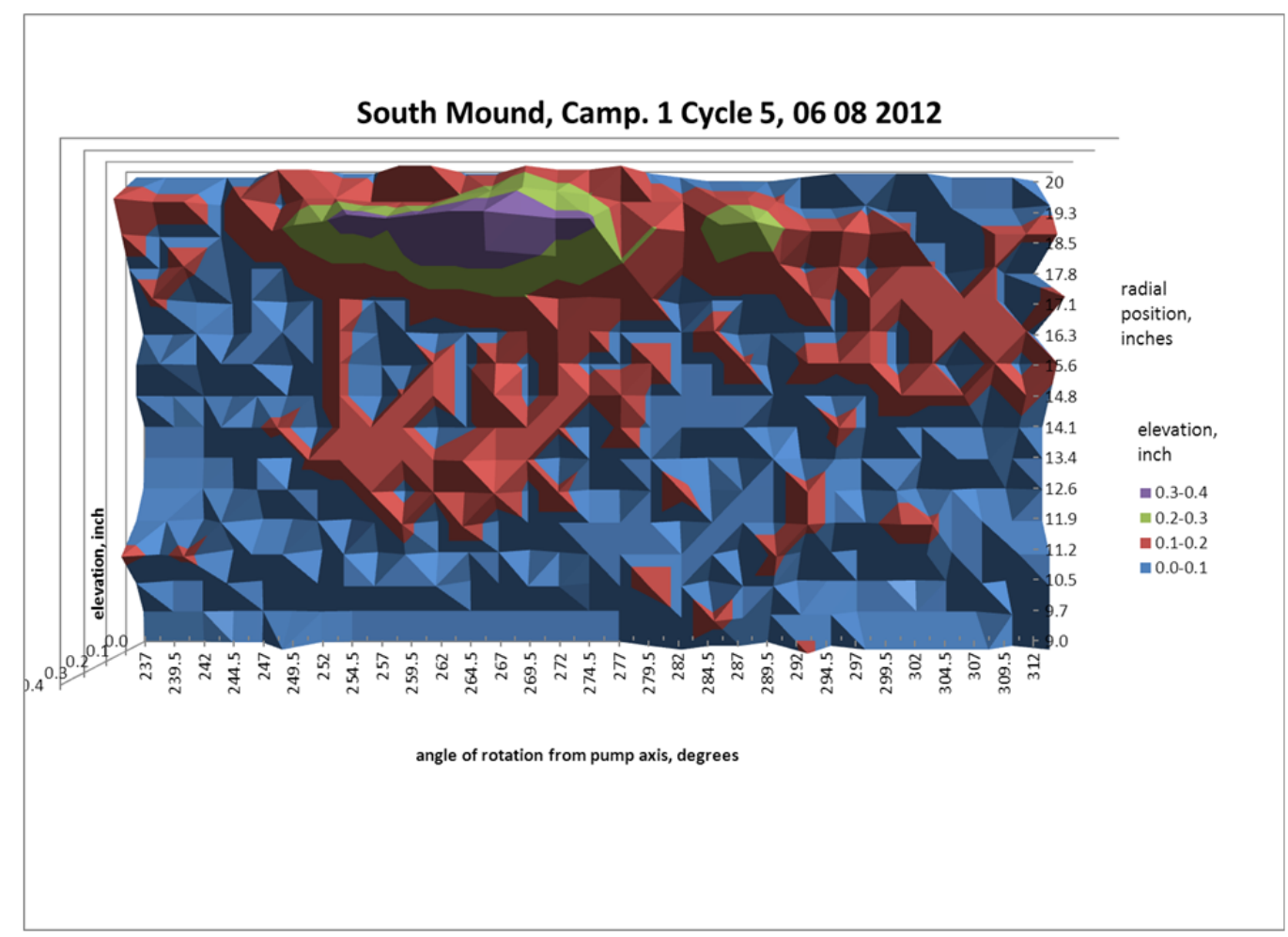

Figure 4-31. South mound top view after Cycle 5 of Campaign 1 - Volume of $14.2 \mathrm{in}^{3}$. 


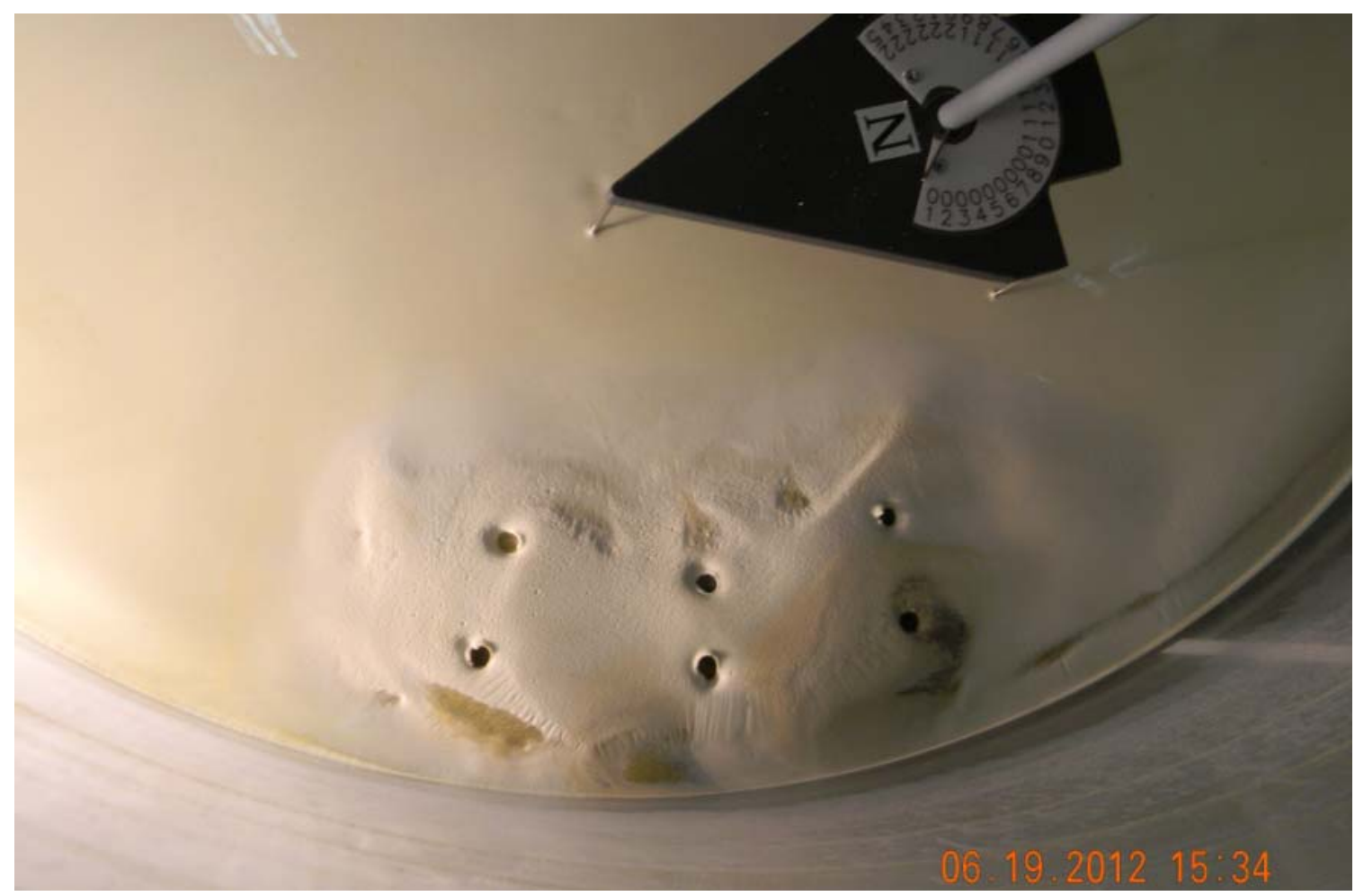

Figure 4-32. Solids North mound after Cycle 10 of Campaign 1.

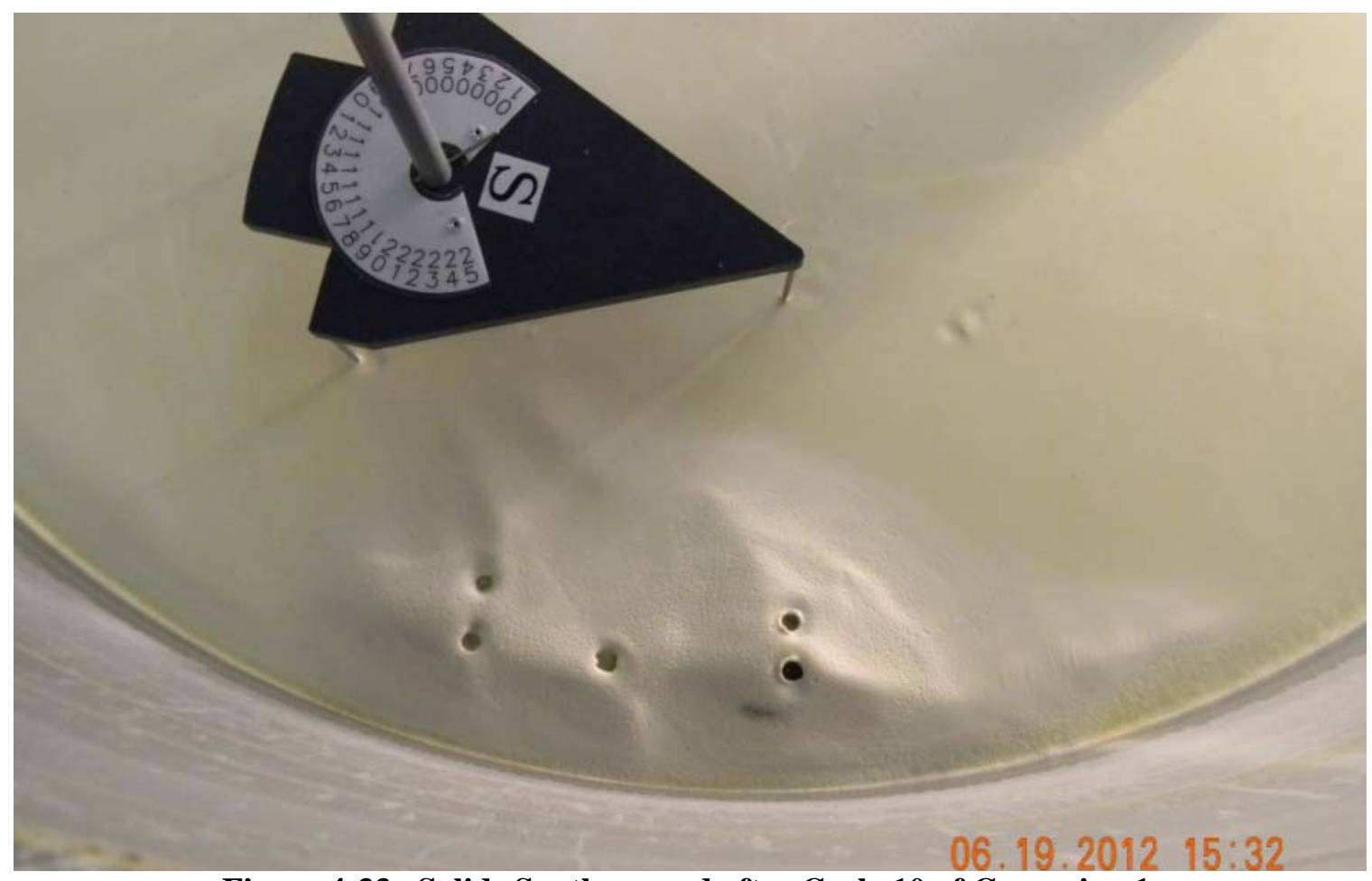

Figure 4-33. Solids South mound after Cycle 10 of Campaign 1. 


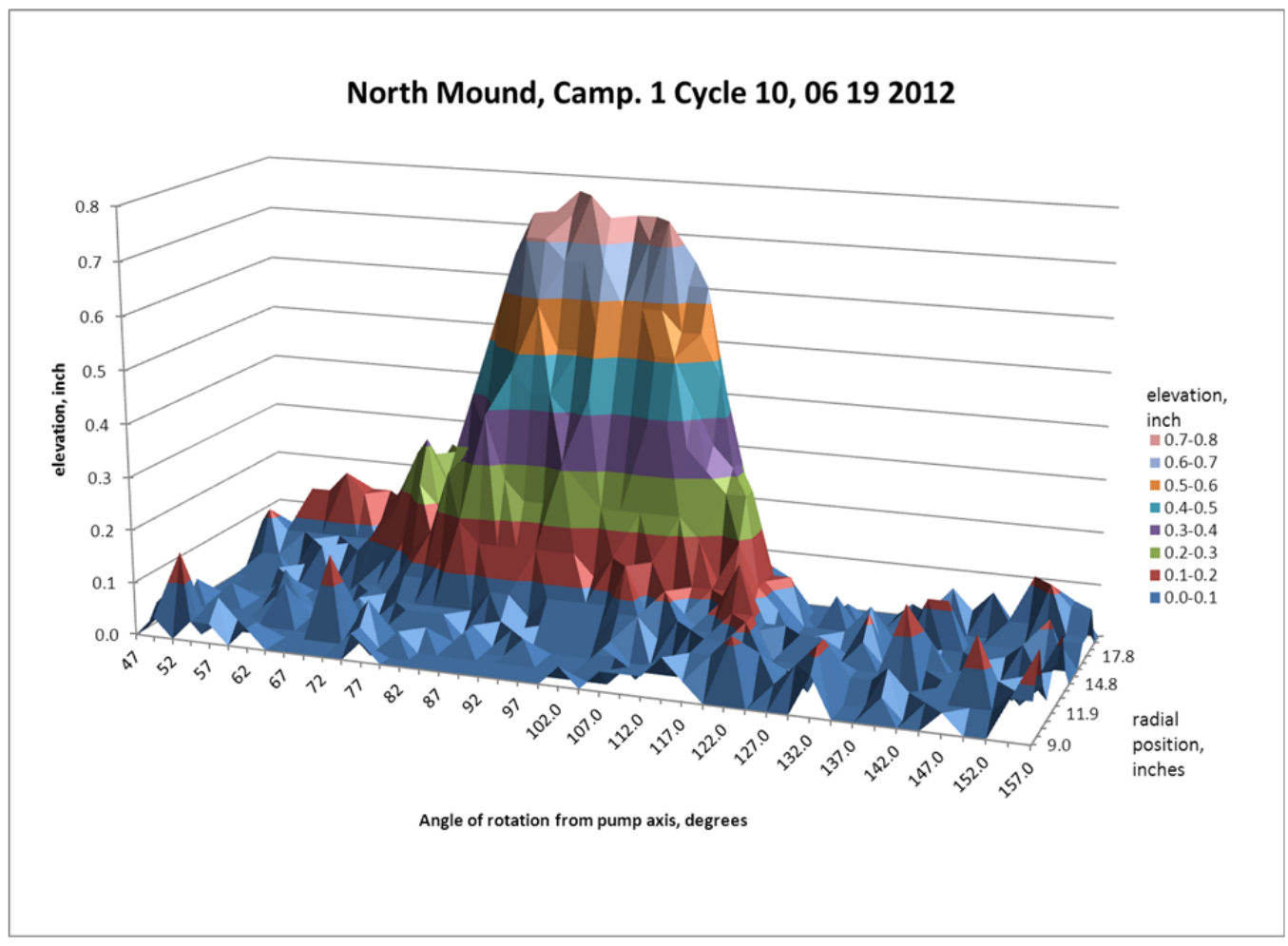

Figure 4-34. North mound side view after Cycle 10 of Campaign 1 - Volume of 37.9 in $^{3}$.

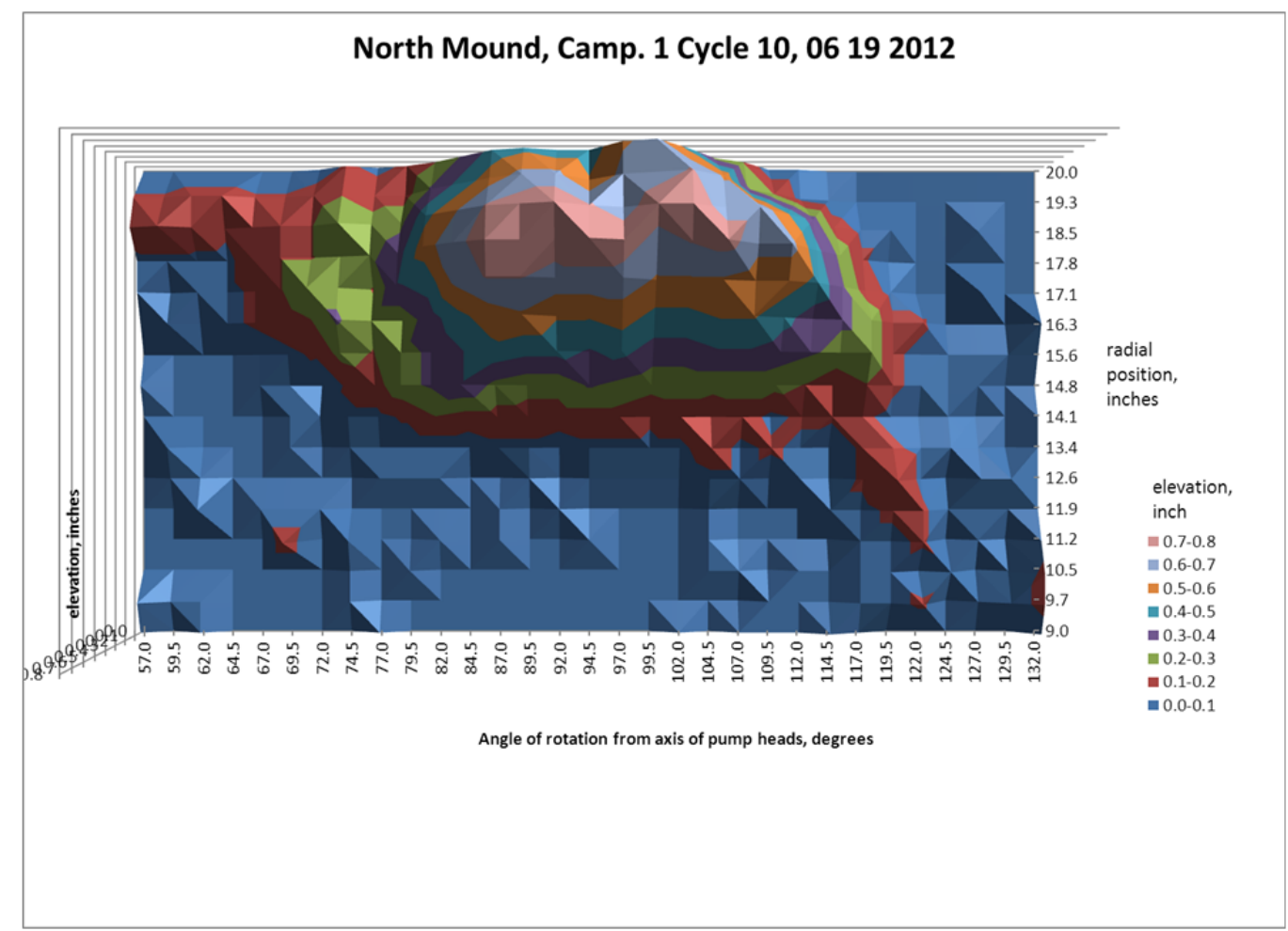

Figure 4-35. North mound top view after Cycle 10 of Campaign 1 - Volume of $37.9 \mathrm{in}^{3}$. 


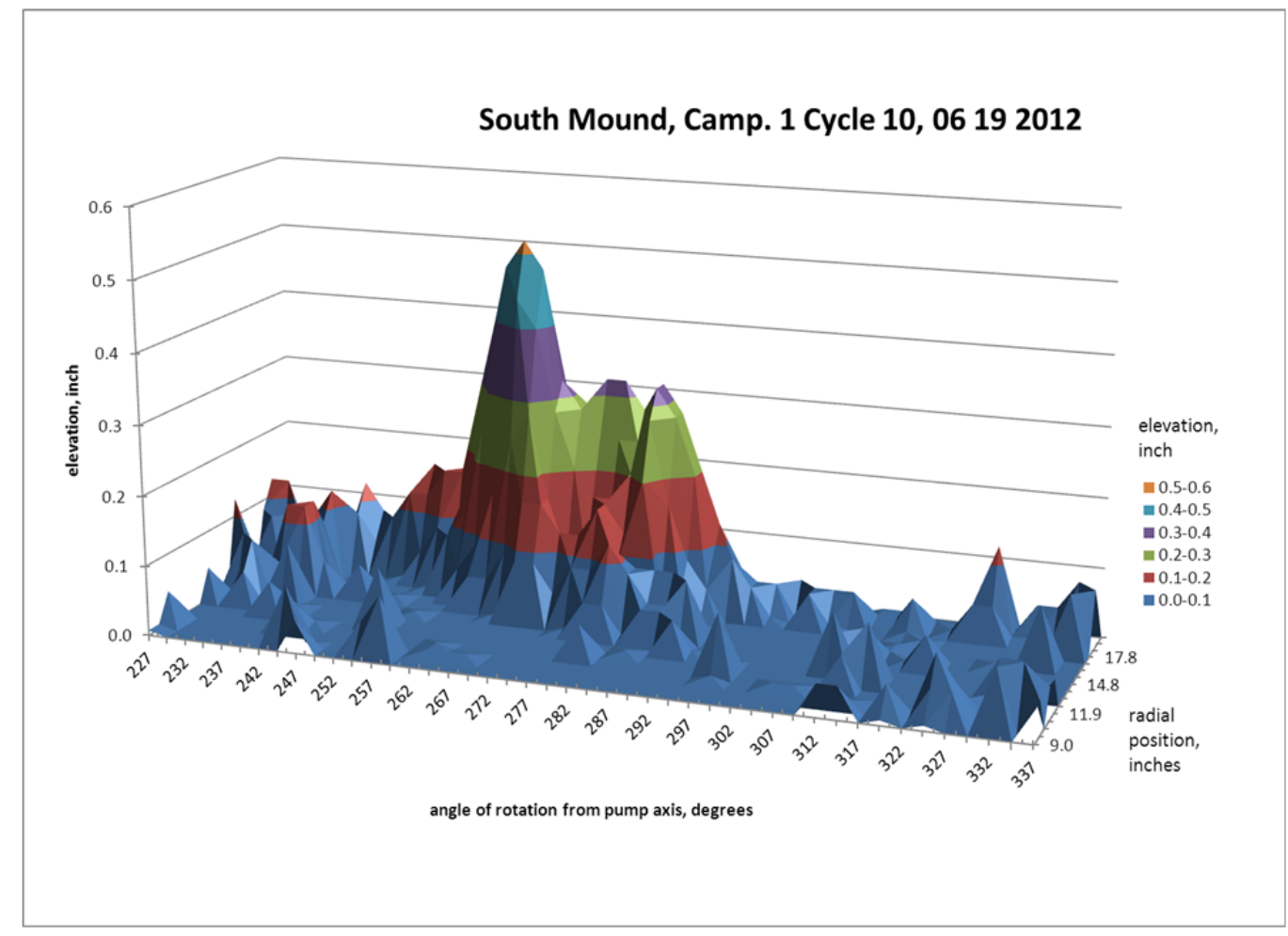

Figure 4-36. South mound side view after Cycle 10 of Campaign 1 - Volume of $13.0 \mathrm{in}^{3}$.

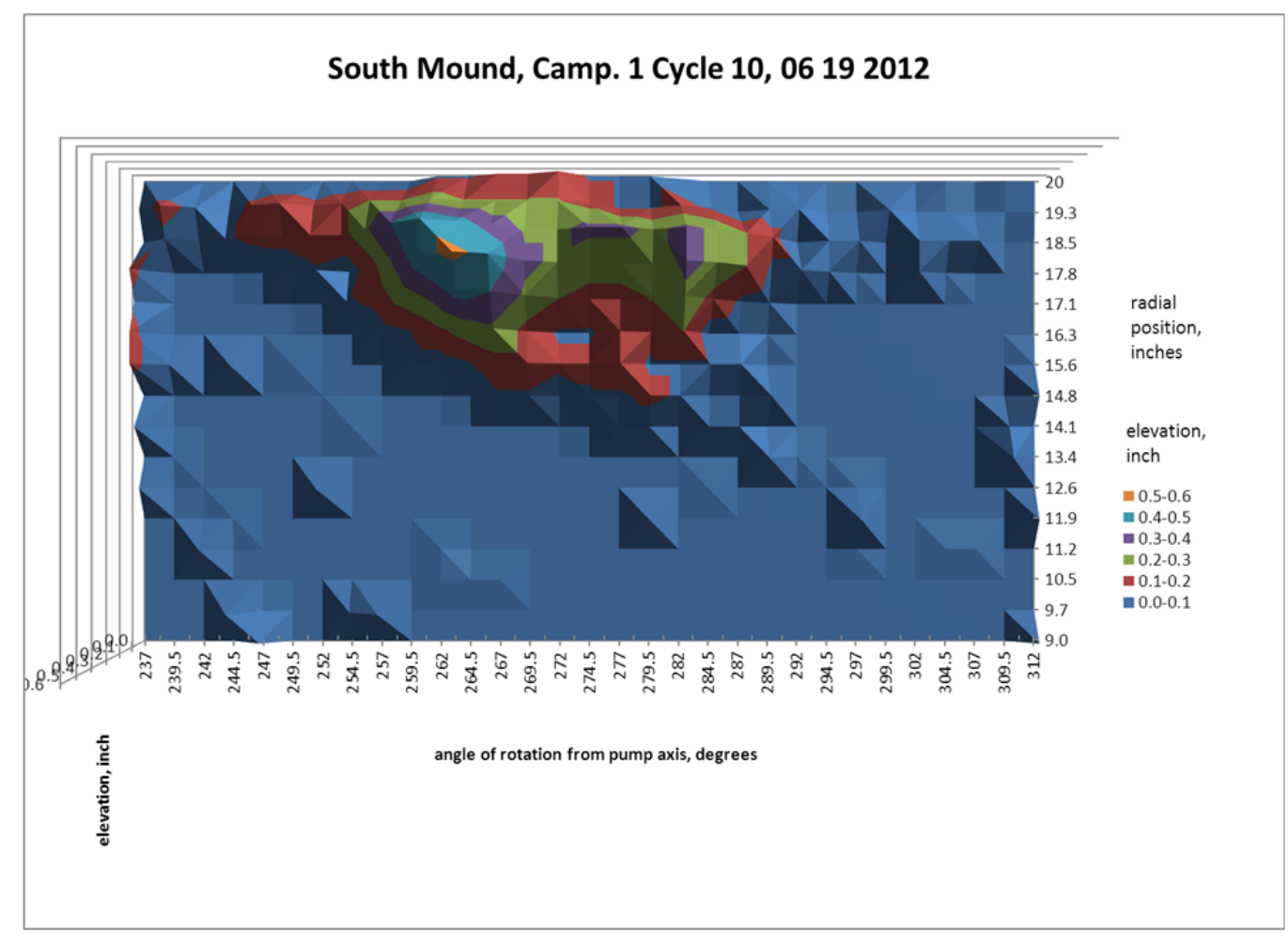

Figure 4-37. South mound top view after Cycle 10 of Campaign 1 - Volume of $13.0 \mathrm{in}^{3}$. 
Revision 0

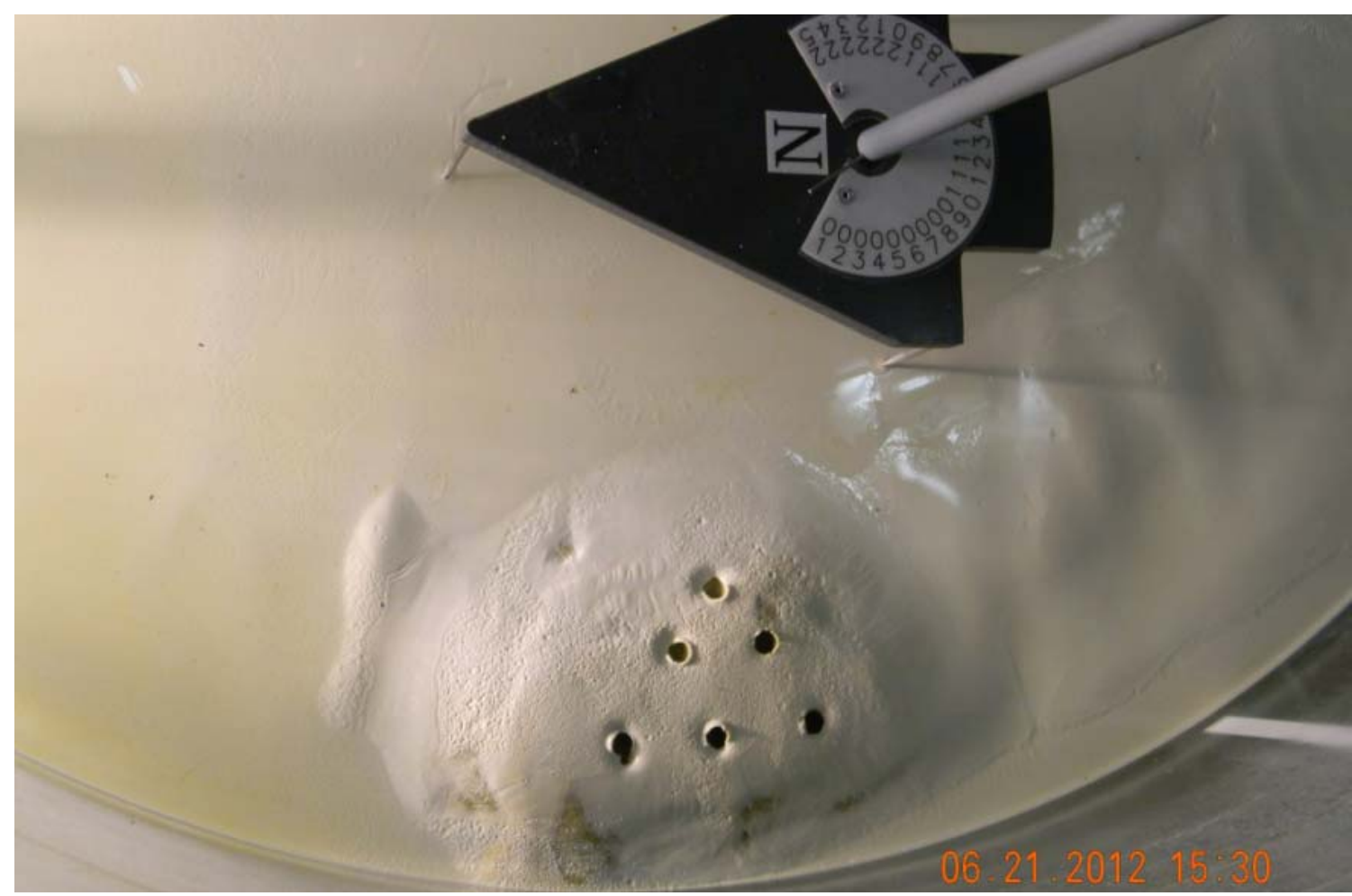

Figure 4-38. Solids North mound after Cycle 1 of Campaign 2.

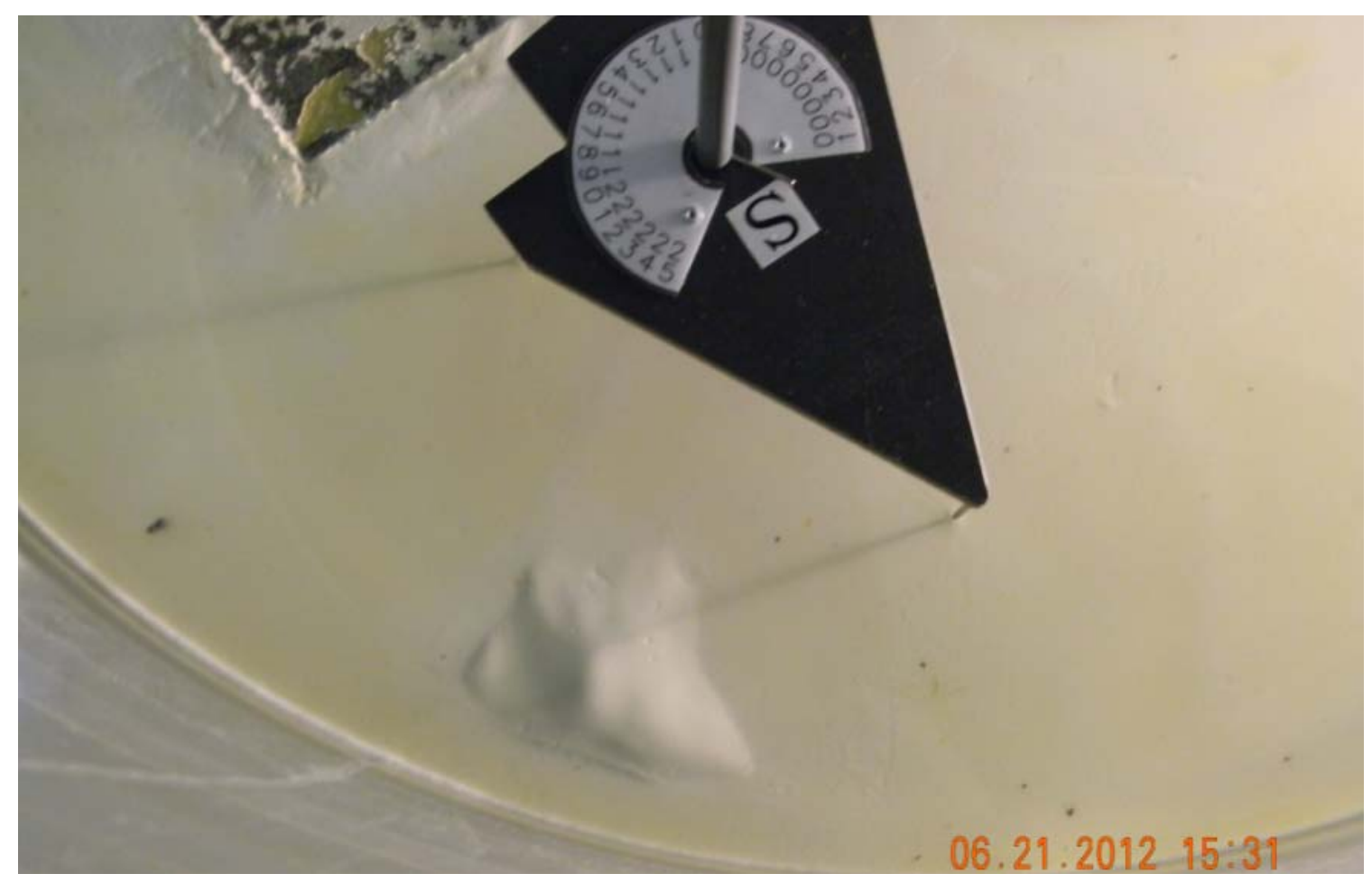

Figure 4-39. Solids South mound after Cycle 1 of Campaign 2. 


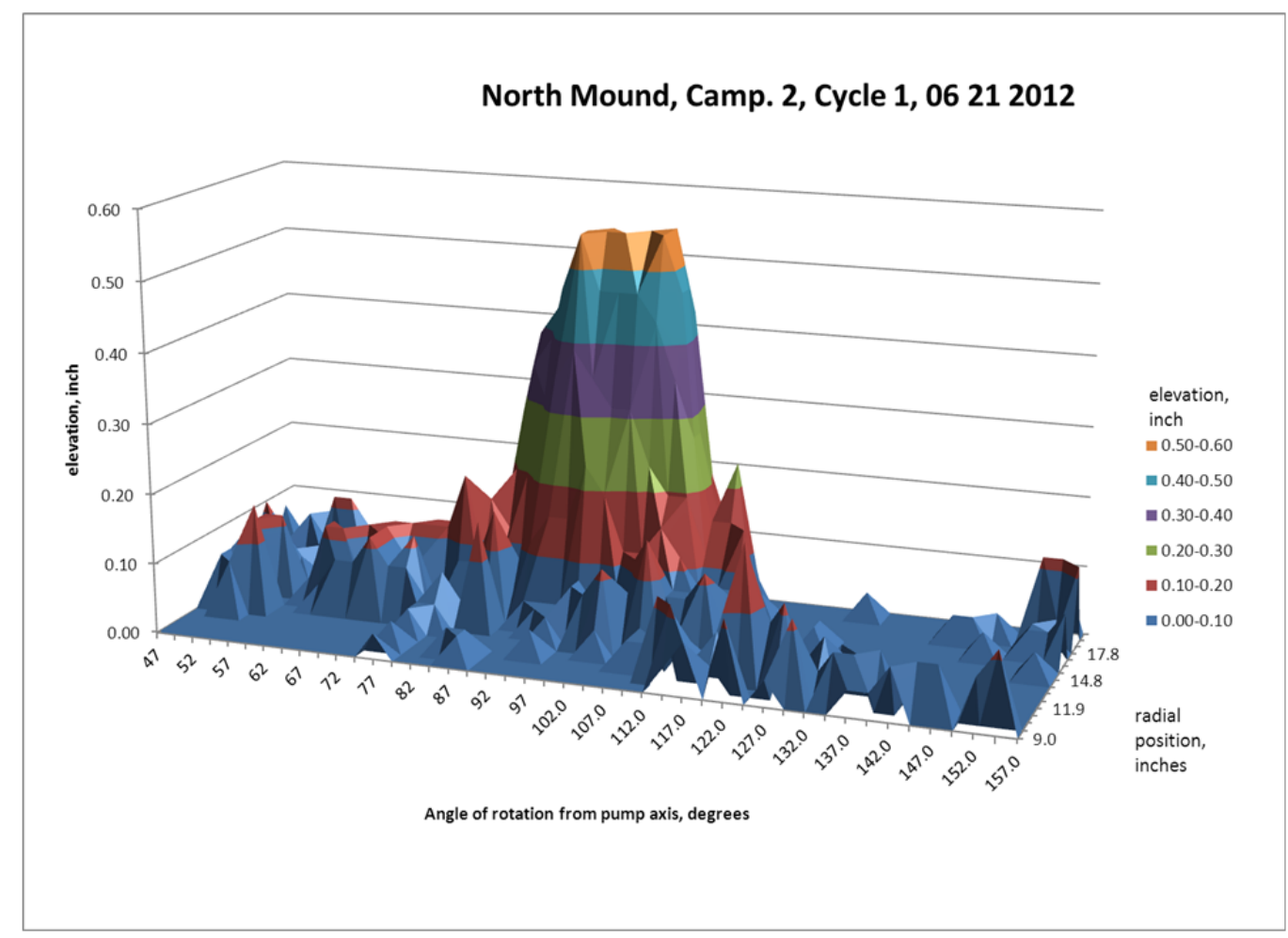

Figure 4-40. North mound side view after Cycle 1 of Campaign 2 - Volume of 19.4 in $^{3}$.

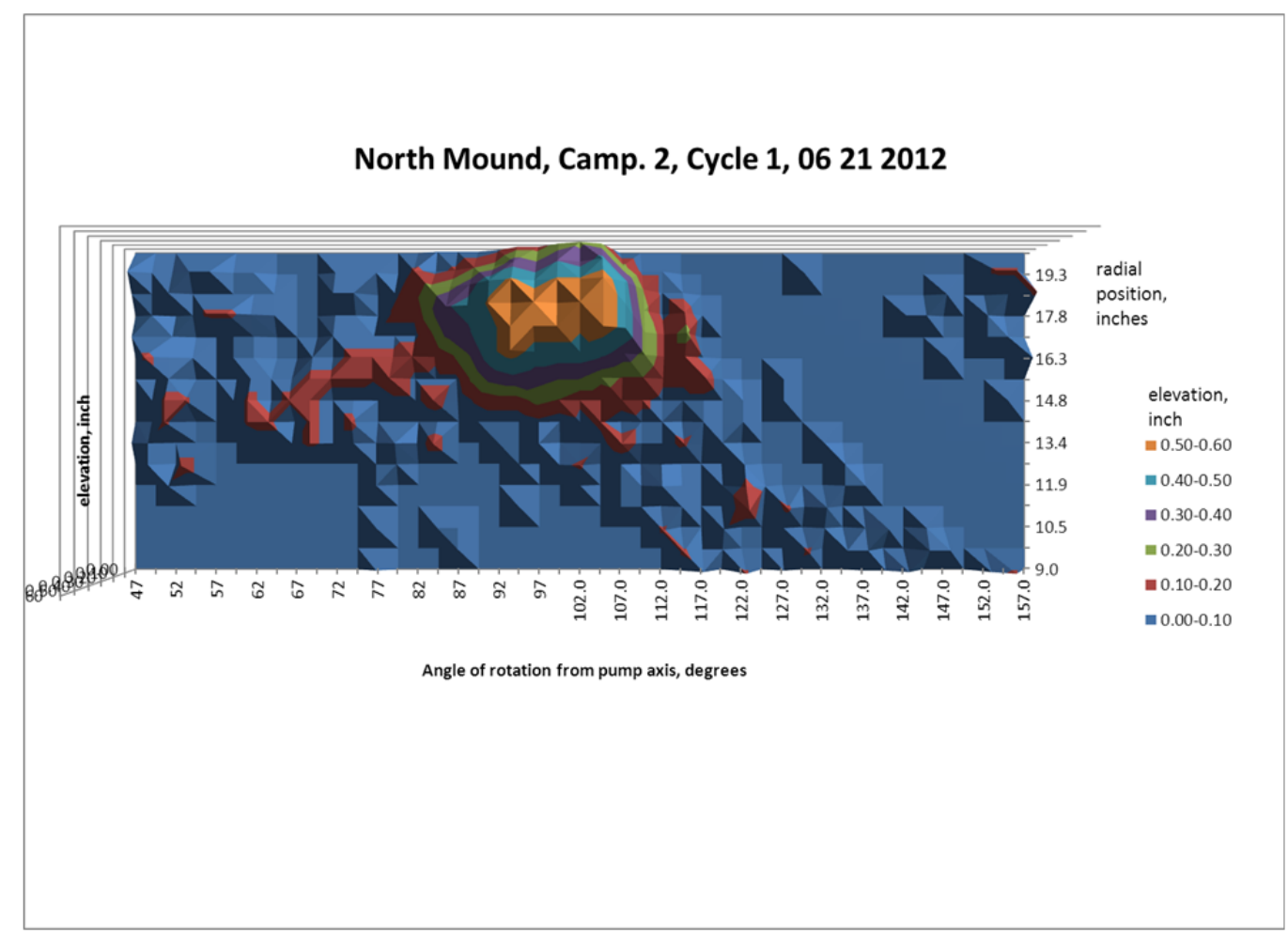

Figure 4-41. North mound top view after Cycle 1 of Campaign 2 - Volume of $19.4 \mathrm{in}^{3}$. 


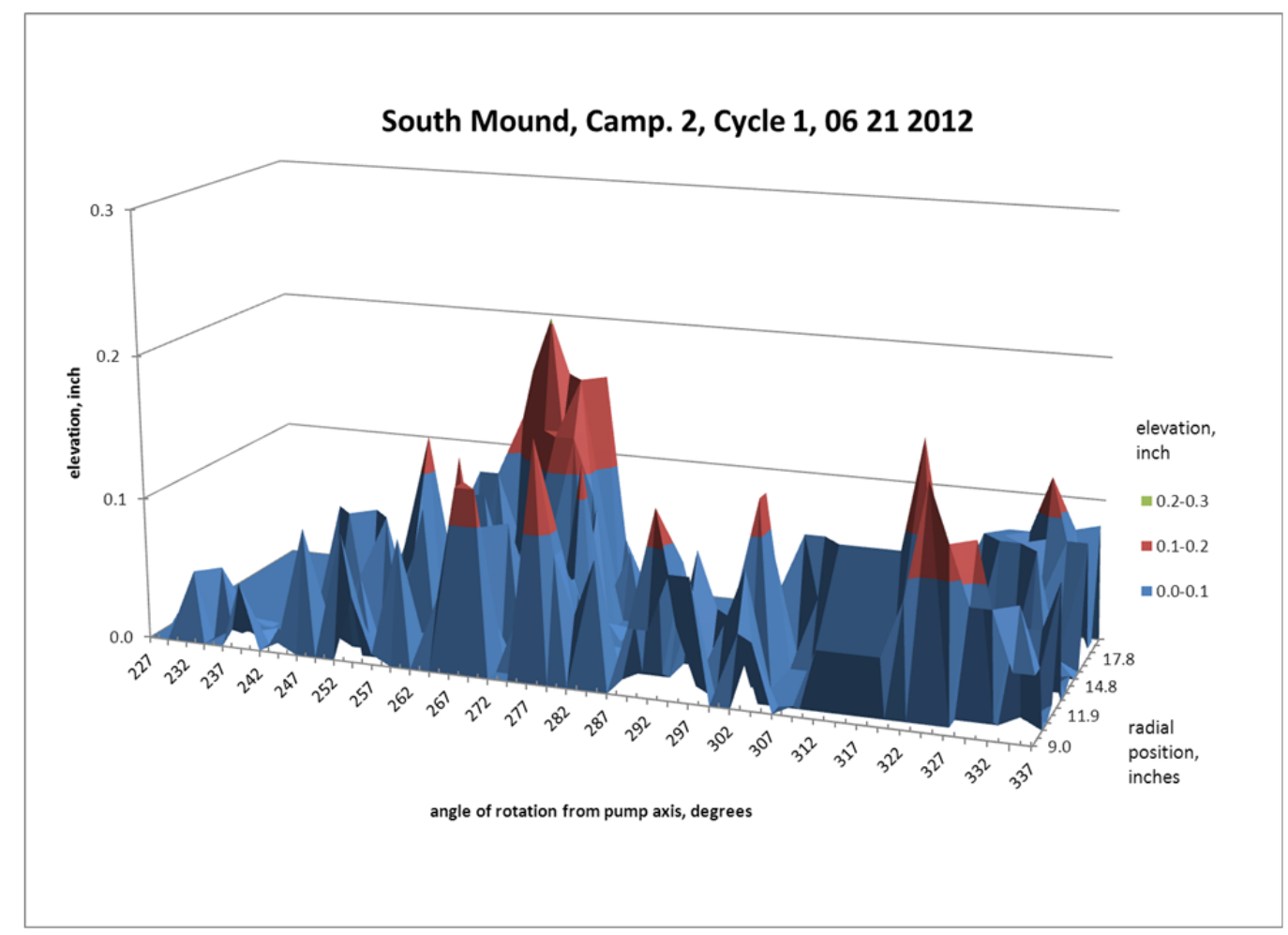

Figure 4-42. South mound side view after Cycle 1 of Campaign 2 - Volume of $2.0 \mathrm{in}^{3}$.

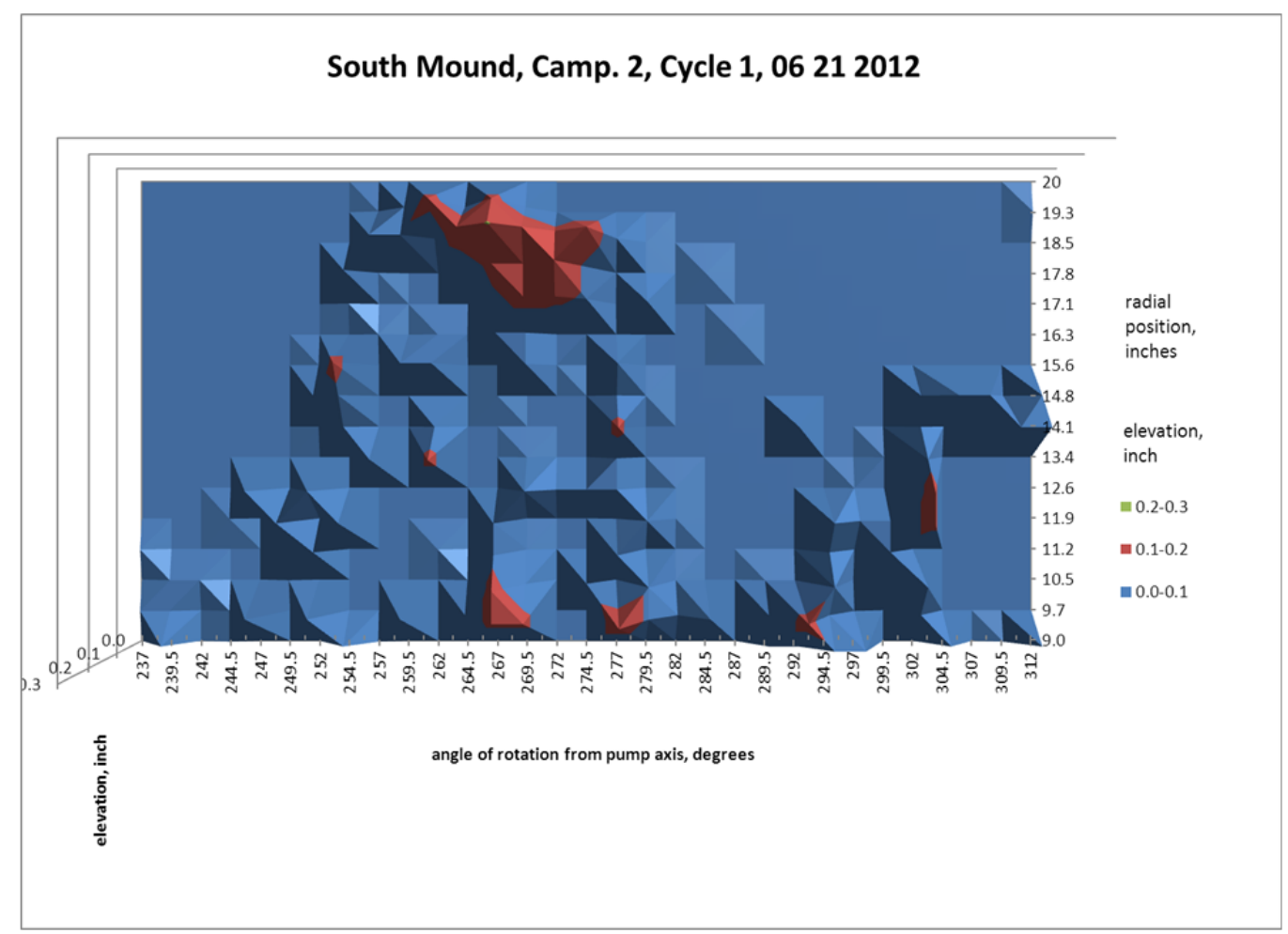

Figure 4-43. South mound top view after Cycle 1 of Campaign 2 - Volume of 2.0 in $^{3}$. 


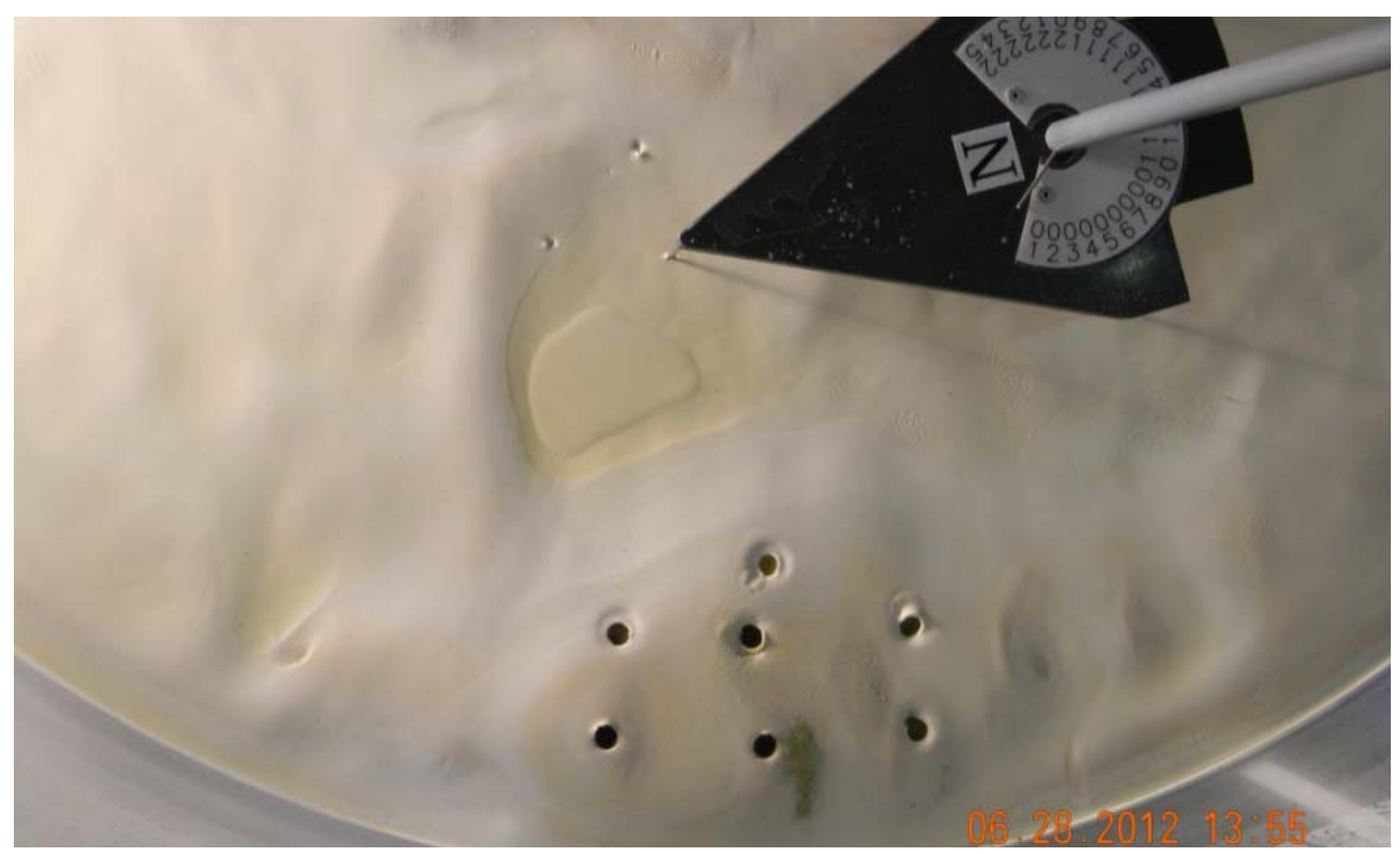

Figure 4-44. Solids North mound after Cycle 5 of Campaign 2.

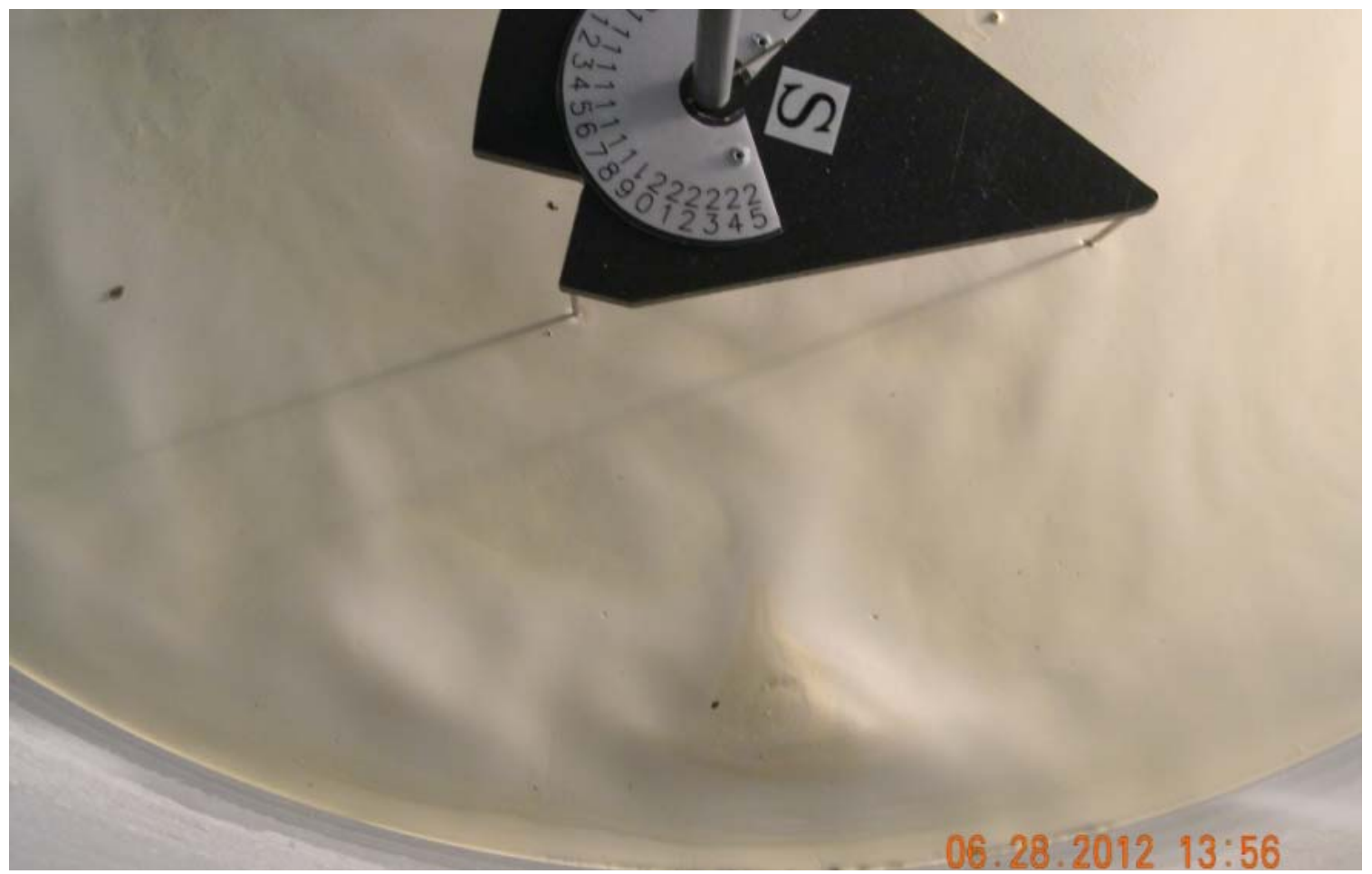

Figure 4-45. Solids South mound after Cycle 5 of Campaign 2. 


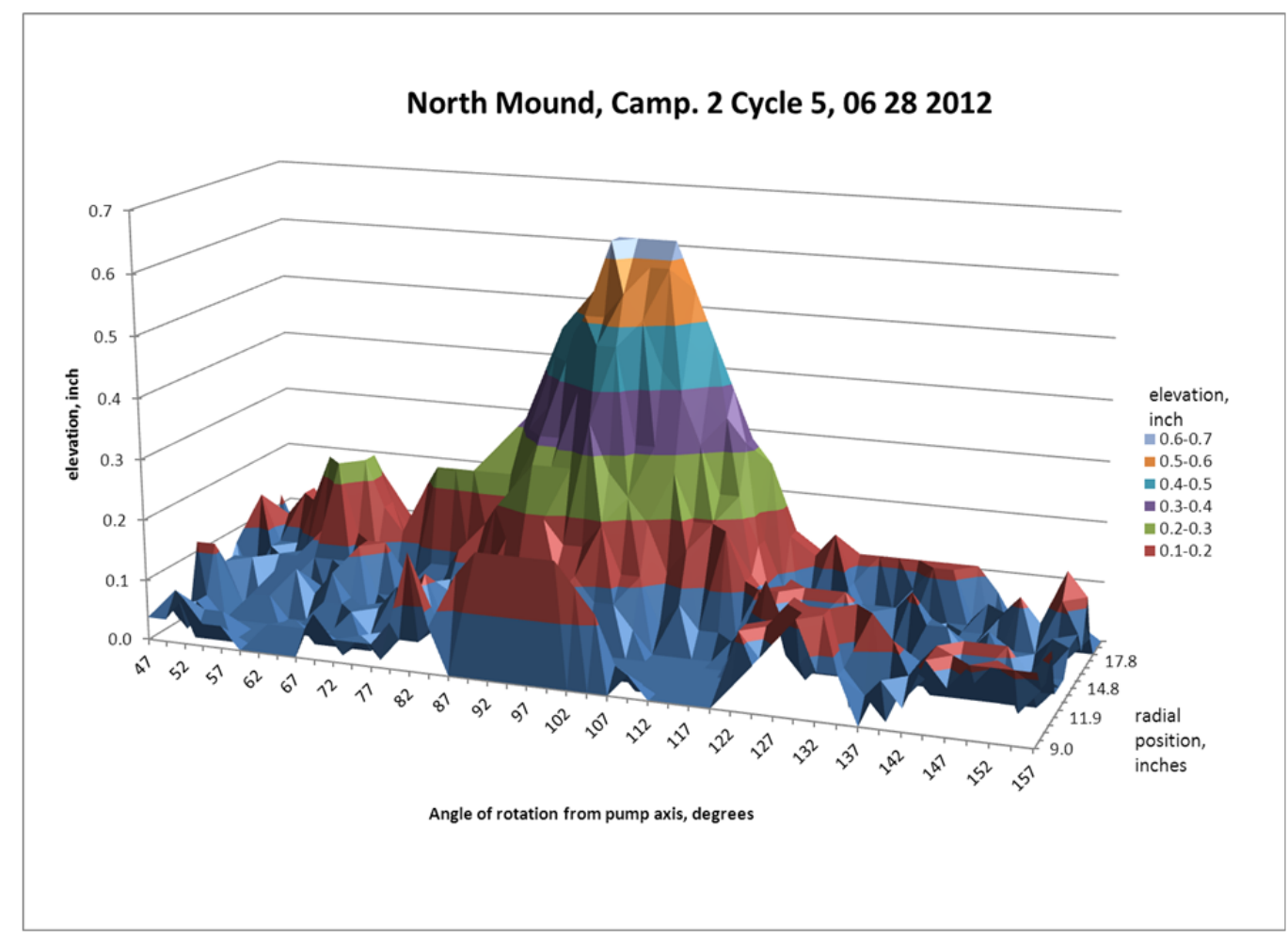

Figure 4-46. North mound side view after Cycle 5 of Campaign 2 - Volume of 30.9 in $^{3}$.

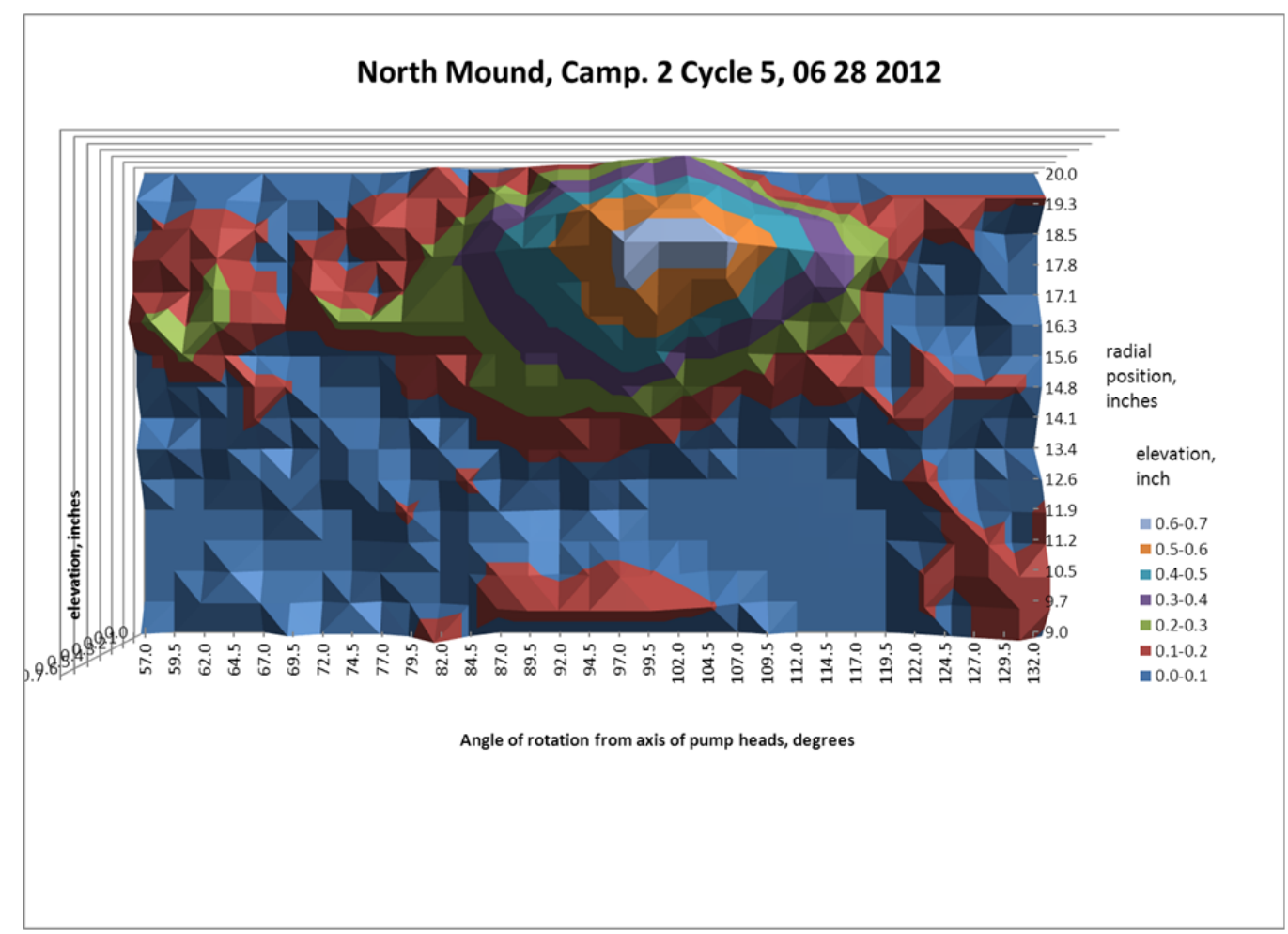

Figure 4-47. North mound top view after Cycle 5 of Campaign 2 - Volume of $30.9 \mathrm{in}^{3}$. 


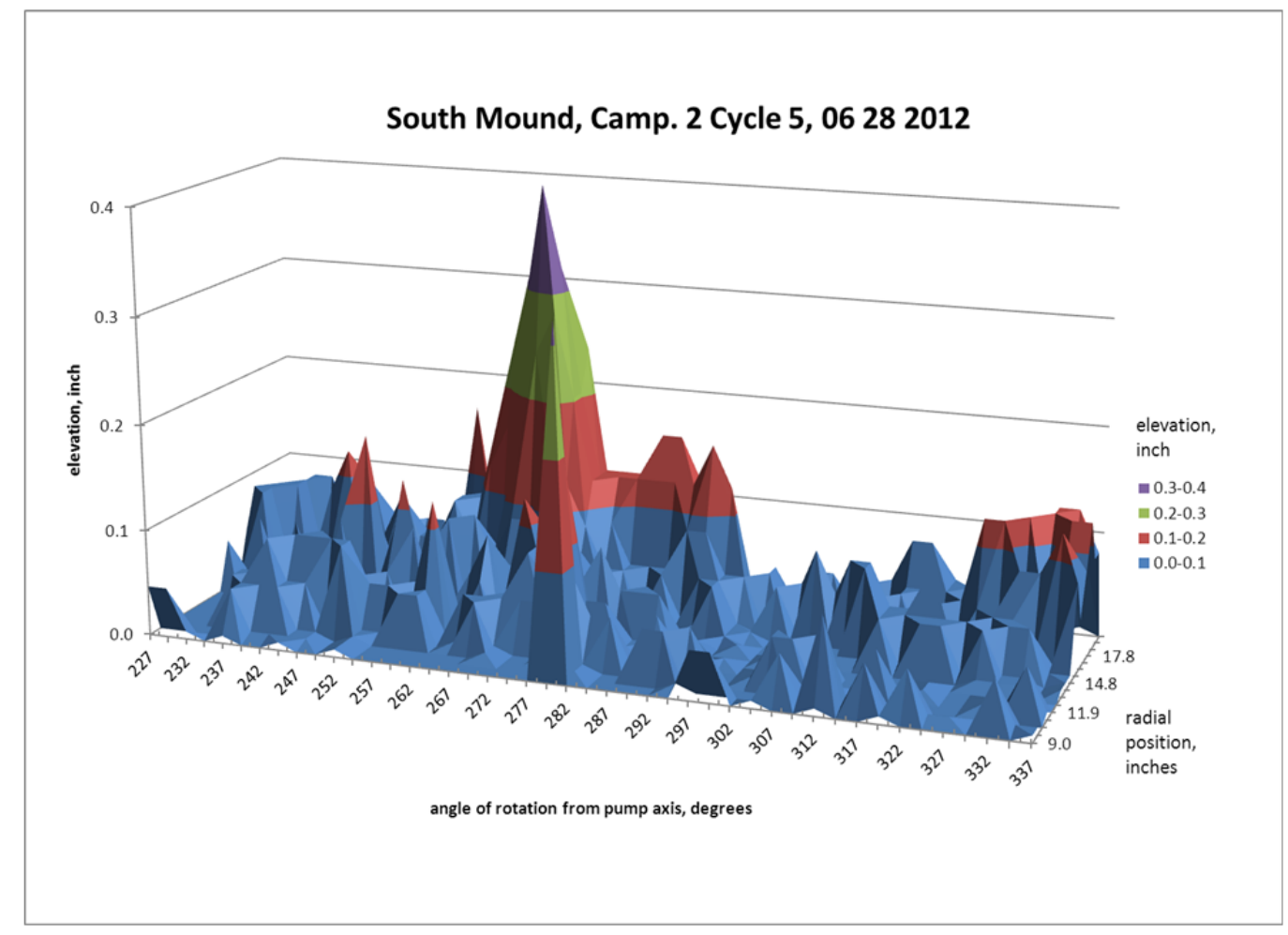

Figure 4-48. South mound side view after Cycle 5 of Campaign 2 - Volume of 6.4 in $^{3}$.

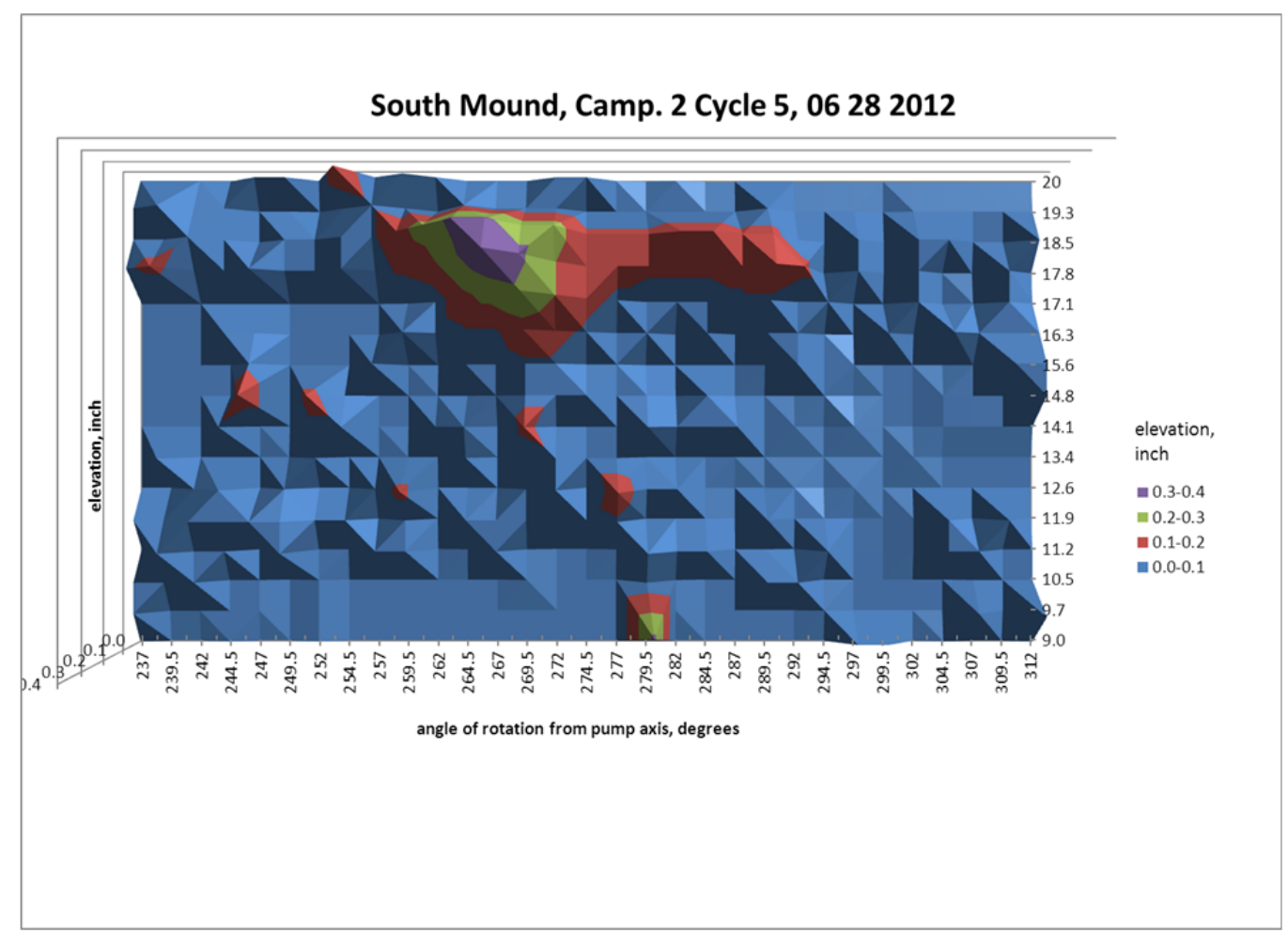

Figure 4-49. South mound top view after Cycle 5 of Campaign 2 - Volume of 6.4 in $^{3}$. 
Revision 0

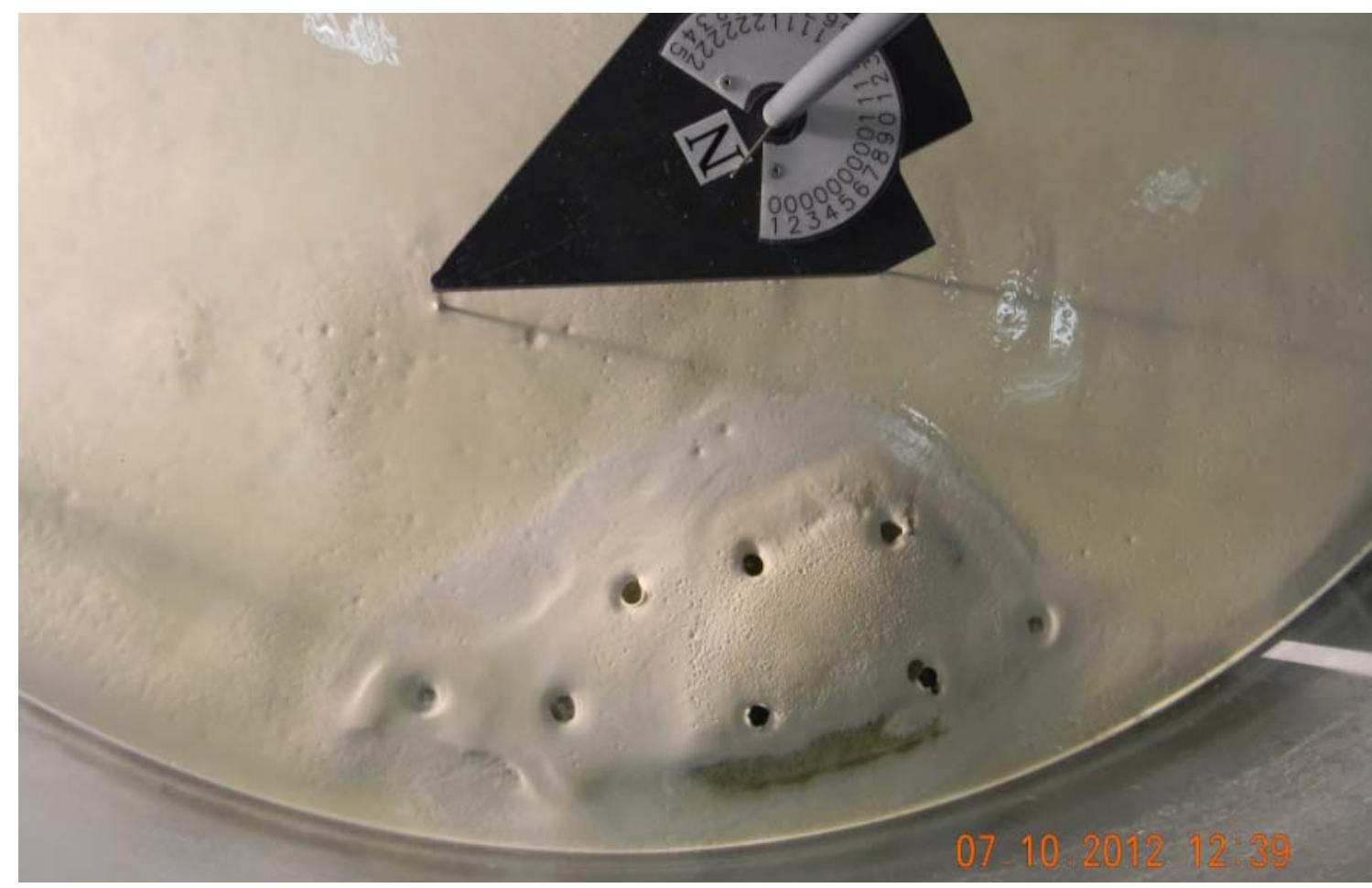

Figure 4-50. Solids North mound after Cycle 10 of Campaign 2.

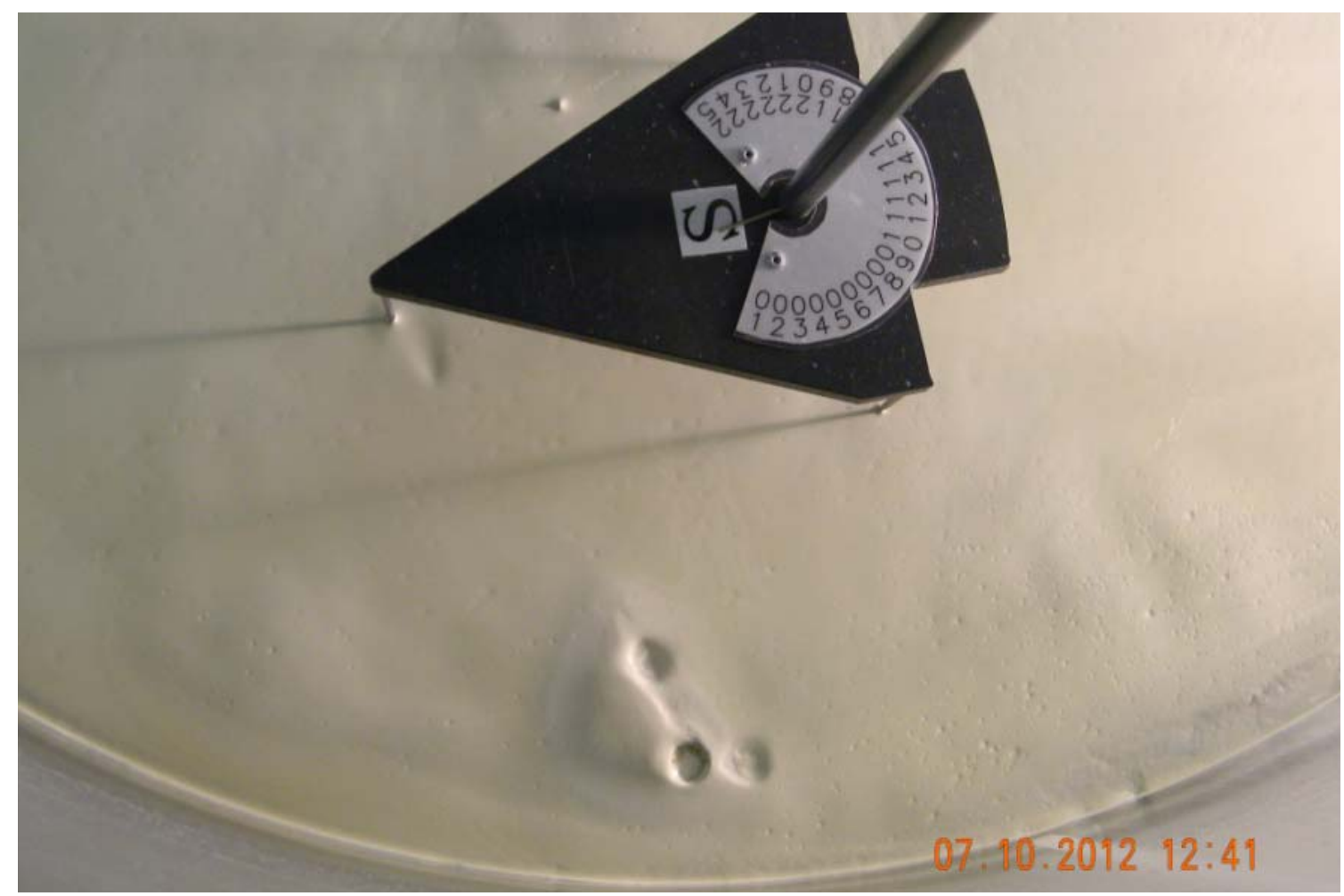

Figure 4-51. Solids South mound after Cycle 10 of Campaign 2. 


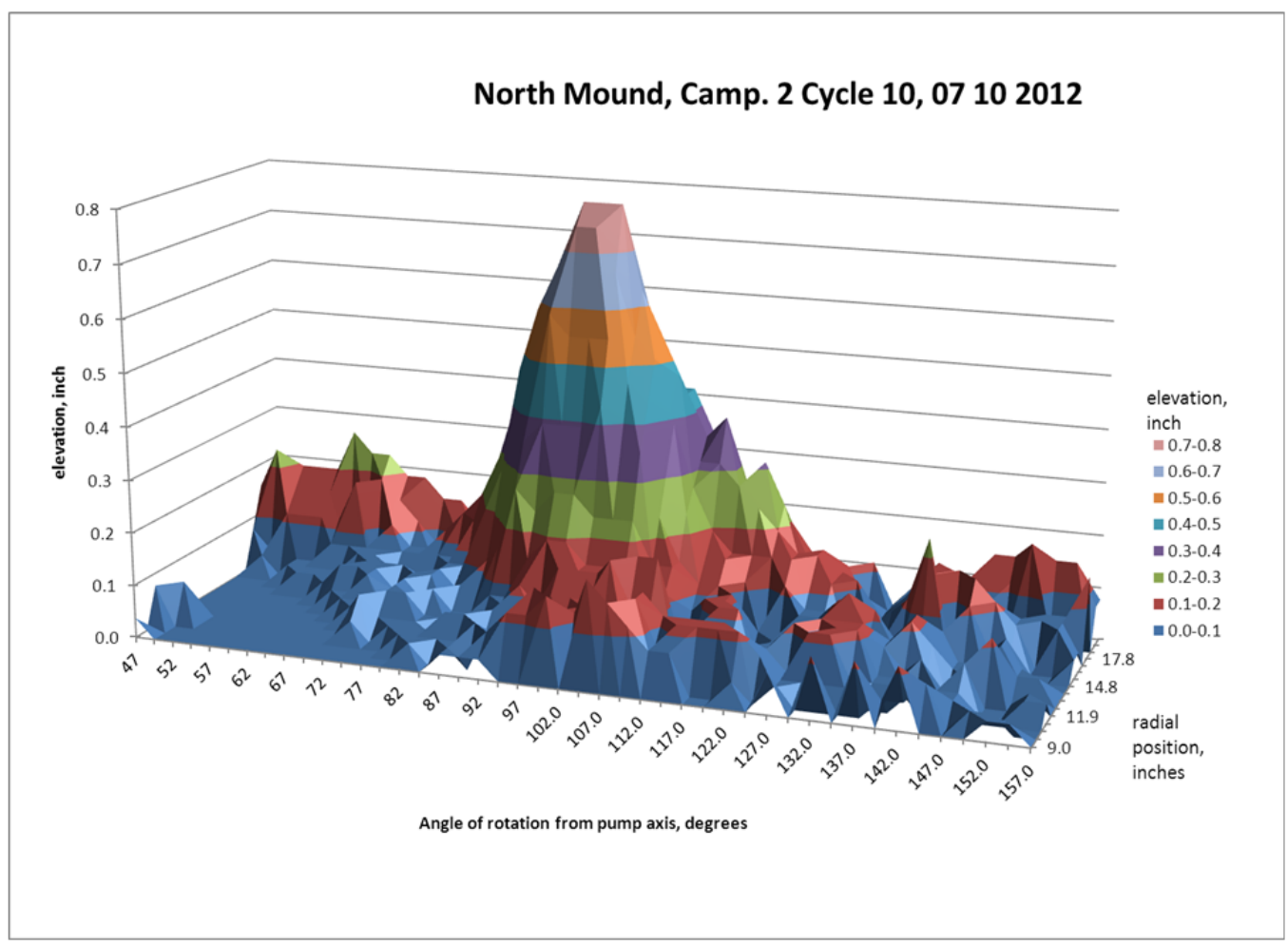

Figure 4-52. North mound side view after Cycle 10 of Campaign 2 - Volume of $35.4 \mathrm{in}^{3}$.

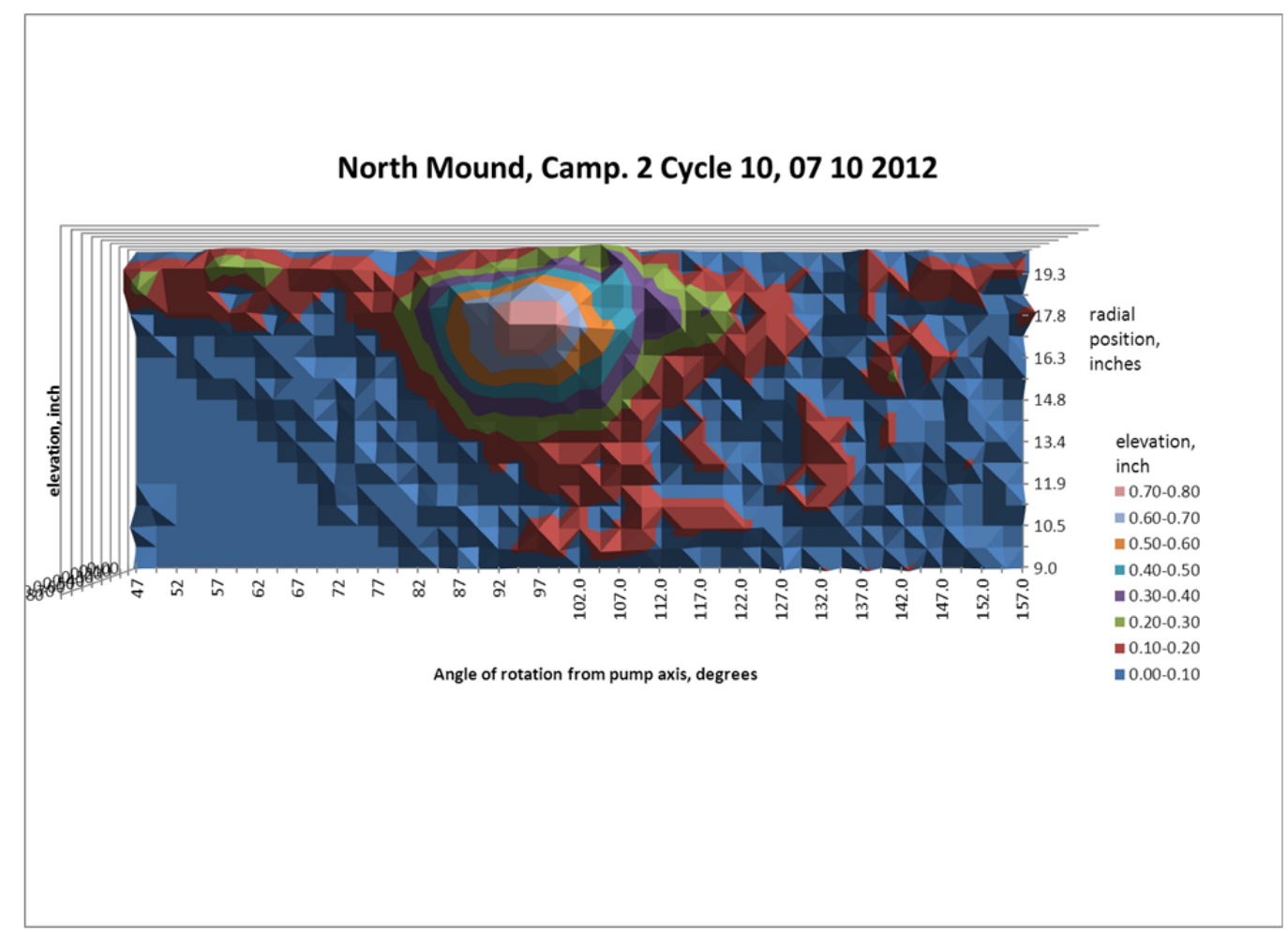

Figure 4-53. North mound top view after Cycle 10 of Campaign 2 - Volume of $35.4 \mathrm{in}^{3}$. 


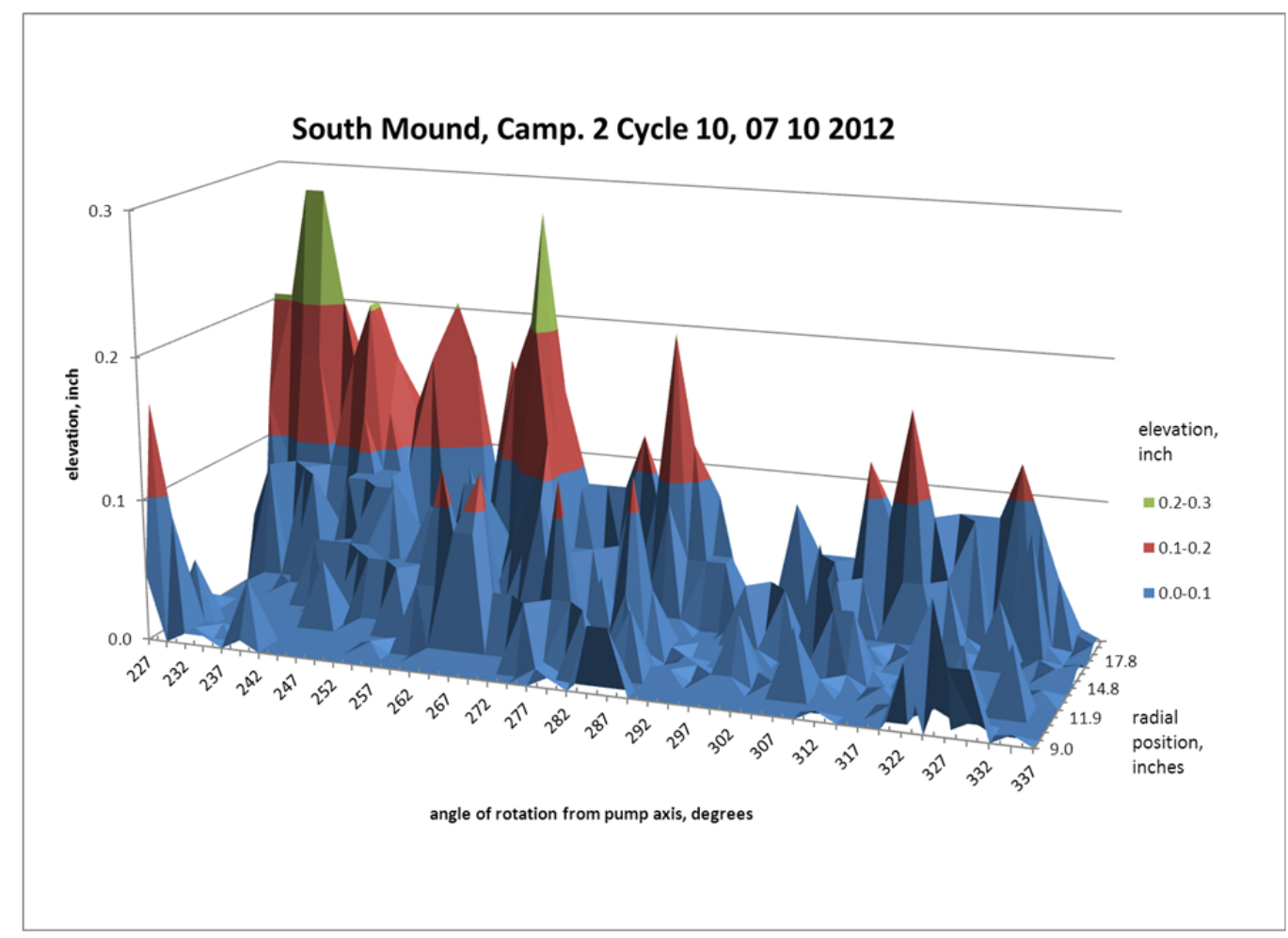

Figure 4-54. South mound side view after Cycle 10 of Campaign 2 - Volume of 9.2 in $^{3}$.

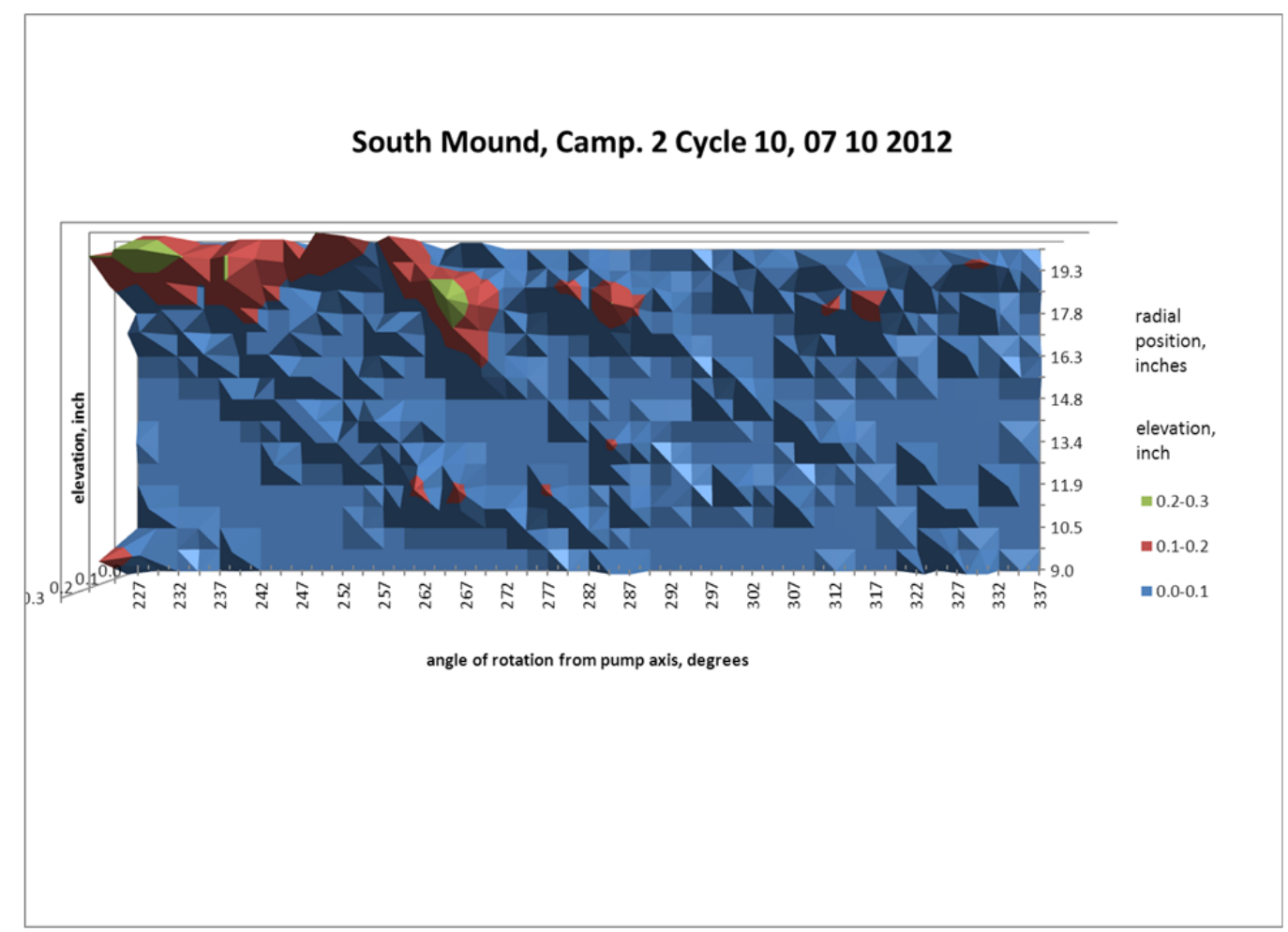

Figure 4-55. South mound top view after Cycle 10 of Campaign 2 - Volume of $9.2 \mathrm{in}^{3}$. 


\subsection{Volume Estimation from Photographic Technique}

Along with laser measurement, a photographic technique was used to measure the volume of the mounds of accumulated solids, as previous explained in Section 3.8.2. The process is straight forward in that a series of photographs is made at predetermined liquid levels and then those pictures are analyzed to determine the area of the exposed mound, i.e., the part of the mound not covered with supernatant. The topographical composite of measured areas and level are then integrated to estimate the volume of the structure. Figure 4-56 shows the volumes obtained and while the North mound data follow the trend of the laser measurements fairly well, the South mound data are more erratic as comparable to those obtained by the laser, shown in Figure 4-19. A comparison of those two data sets will be discussed in the next section; however, the measurement uncertainty for the photographic method is considerably large, e.g.,, $> \pm 20 \%$, because of the small size of the mounds, especially the South mound and the poor visual contrast between the gibbsite/zirconia covered mound surfaces and the simulant.

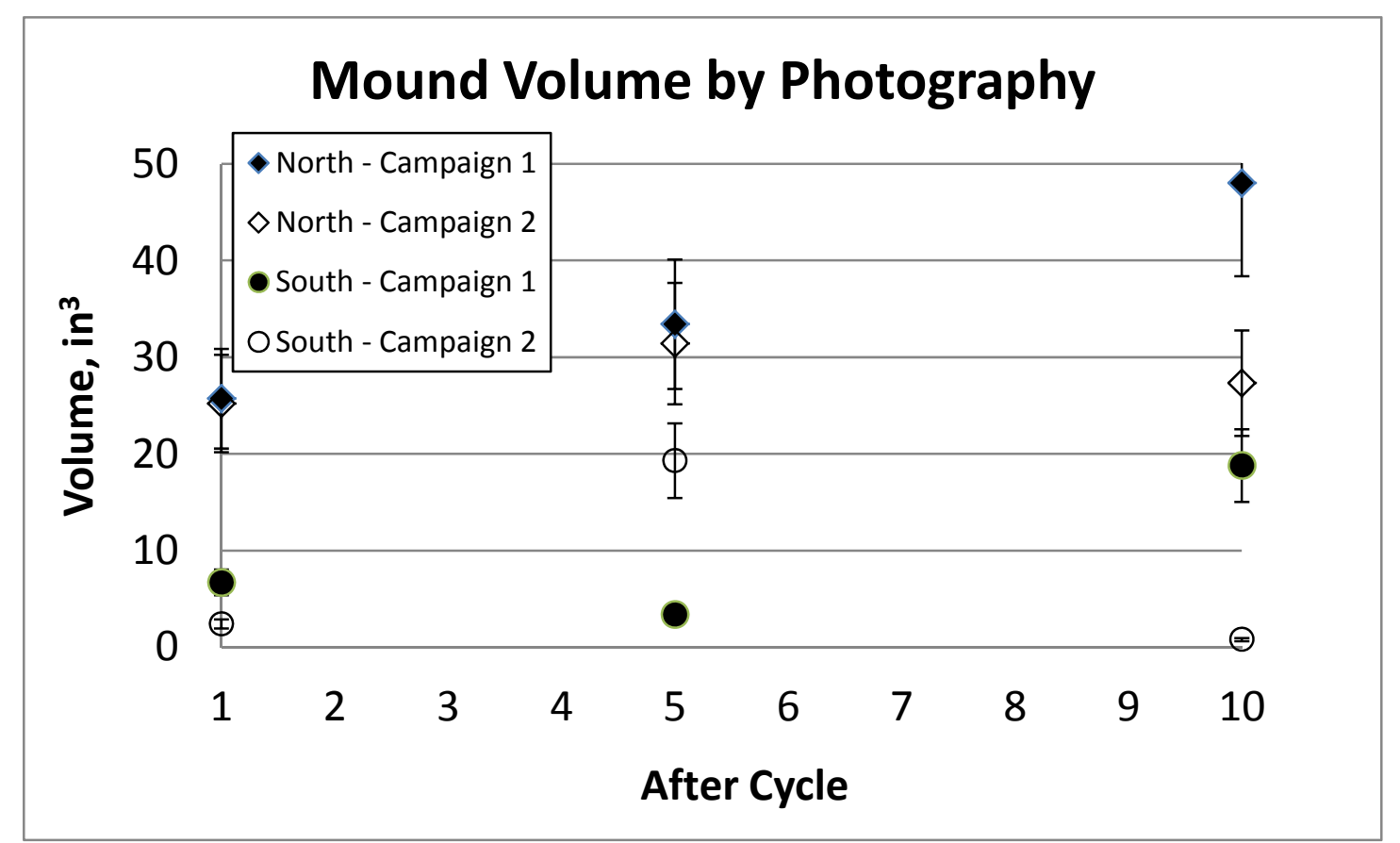

Figure 4-56. Mound volumes obtain from photographic measurements and showing $\pm 20 \%$ uncertainty (uncertainties bars not seen are within data symbols)

The accuracy of the volume obtained with the photographic method is more dependent on the size of the object than the laser technique. Besides this, the accuracy depends on the contrast between the object and the surrounding liquid level. The laser accuracy is just a matter of the knowledge of where the laser point is, which is fairly easy to determine; however, for the photographic technique more judgment necessary by the analyzer. For all of the mound evaluations the process is the same, so the following paragraphs discuss just one of the cycle measurements, specifically the last one, i.e., after Cycle 10 of Campaign 2.

From a series of picture a topographical map of the exposed mound area is constructed as shown in Figure 4-57. On each photograph, Figure 4-58(a) to (g) the "shoreline" must be identified so the exposed area is obtained, which was sometimes difficult. The photographic lighting, the 
angle of view, the liquid-solids contrast, and the proper scaling among all the pictures all play a role in estimating the exposed area. Once all the areas are determined, then the actual liquid level and the number of liquid levels affect the accuracy, which increases as the number of levels increases. For the North mound depicted in Figure 4-57, it had seven levels, because it was about 0.9-inch tall, but the South mound was smaller and had fewer levels.

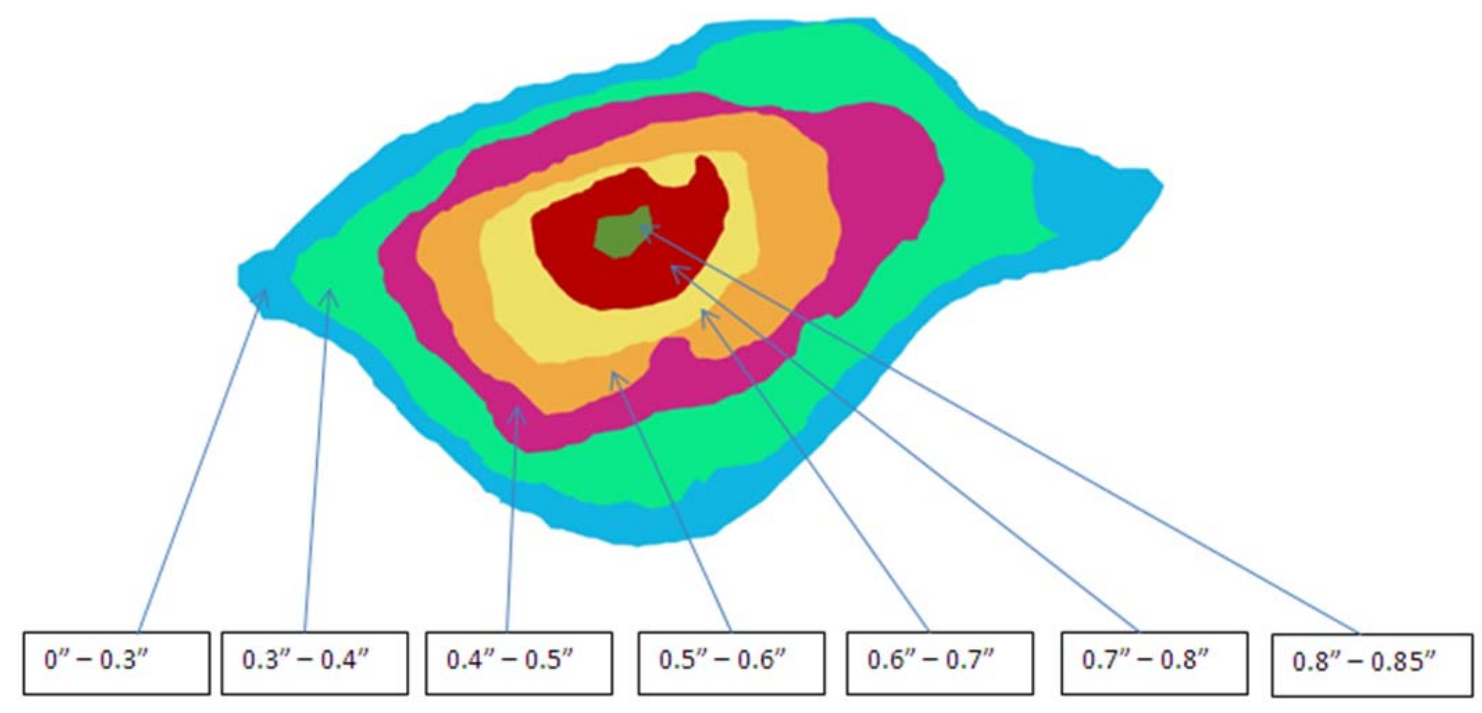

Figure 4-57. Photographic technique: Cycle 10 of Campaign 2 - North Mound Top View

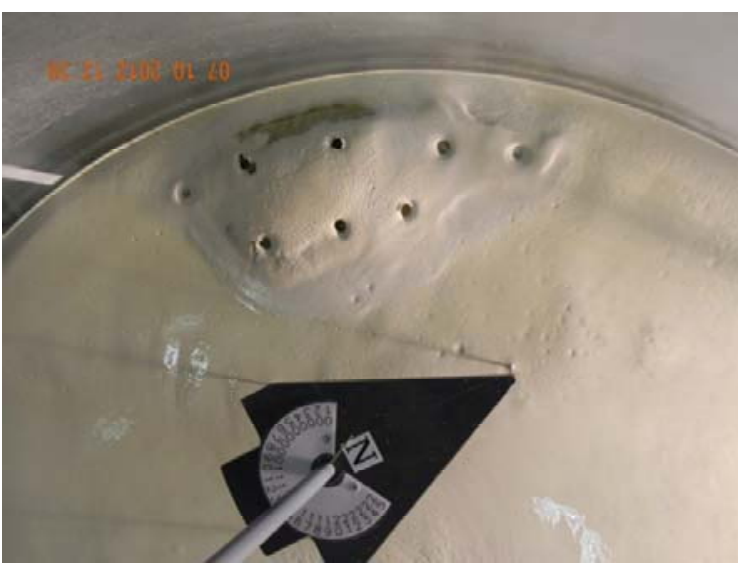

(a) 0.0 inch tank level

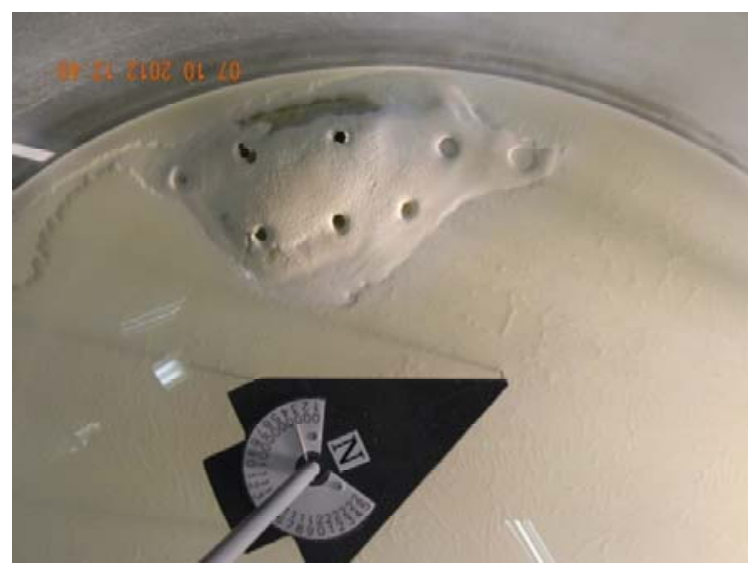

(b) 0.3 inch tank level 
Revision 0

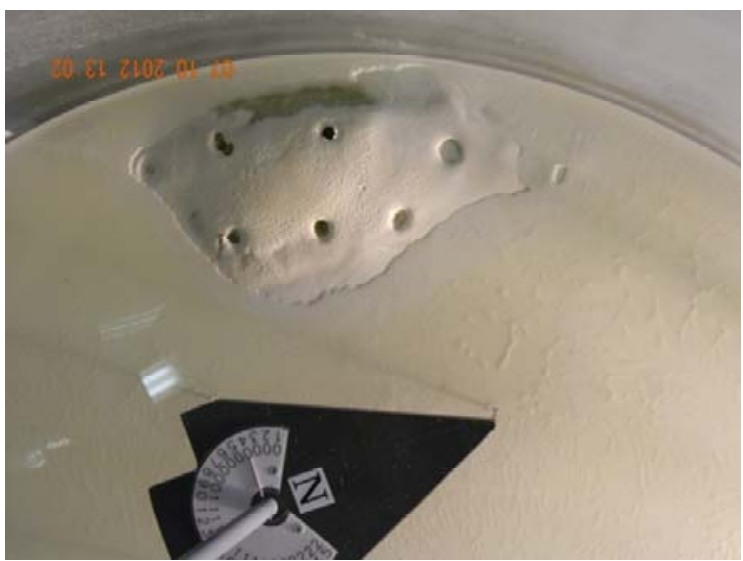

(c) 0.4 inch tank level

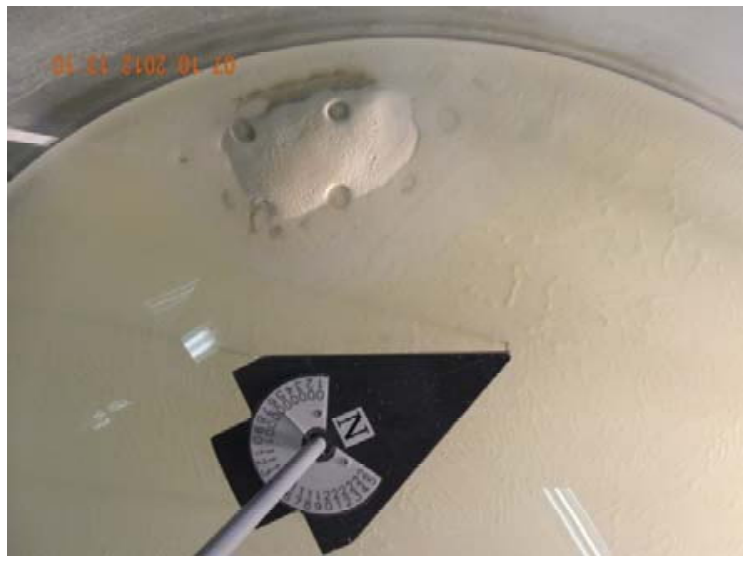

(e) 0.6 inch tank level

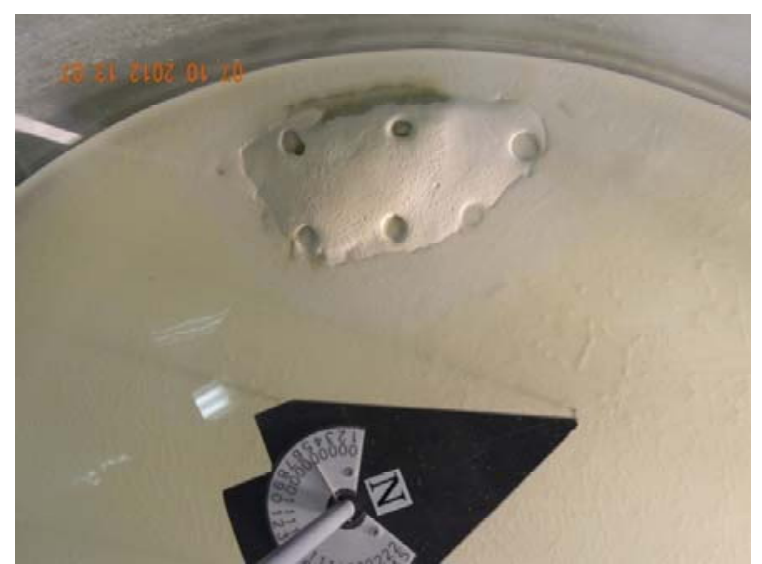

(d) 0.5 inch tank level

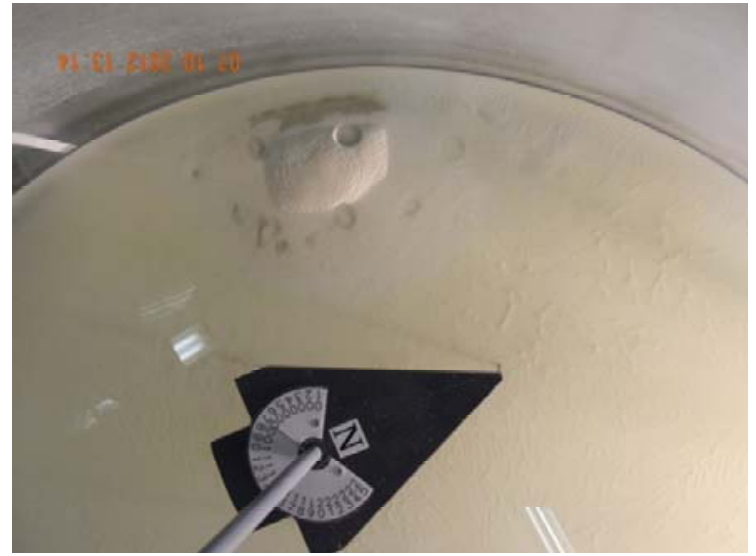

(f) 0.7 inch tank level

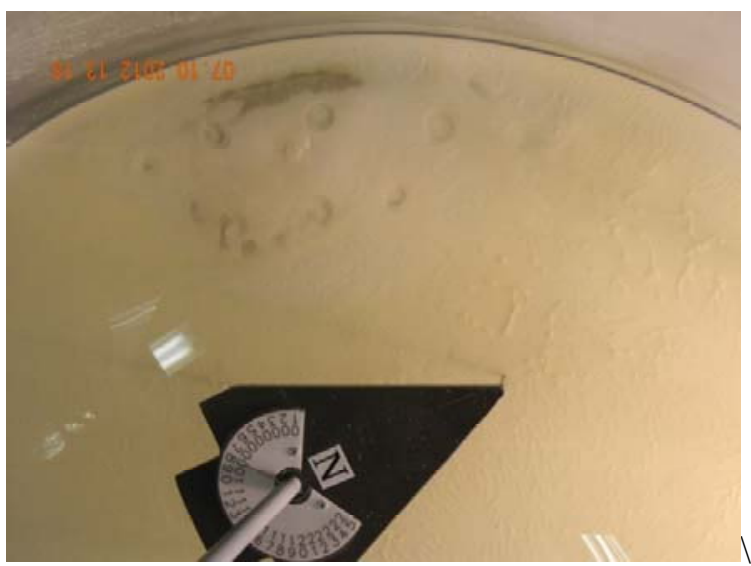

(g) 0.8 inch tank level

Figure 4-58. Photographs of changing tank level to estimate North mound volume: (a) to (g) 
Figure 4-59 shows the South mound of Cycle 10 of Campaign 2. It was just under 0.5 -inch in height and as can be seen from the photographs, Figure 4-60(a) to (c) only two liquid levels above the tank bottom showed an exposed mound surface. There is insufficient information to assemble an accurate composite to estimate the volume, so the volume uncertainty is much more than the $20 \%$ estimated for the North mound.

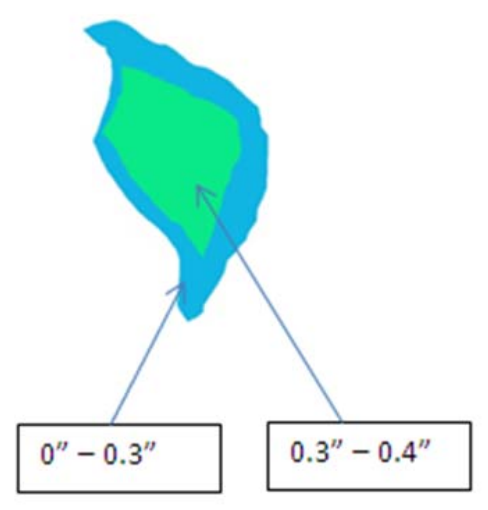

Figure 4-59. Photographic technique: Cycle 10 of Campaign 2 - South Mound Top View

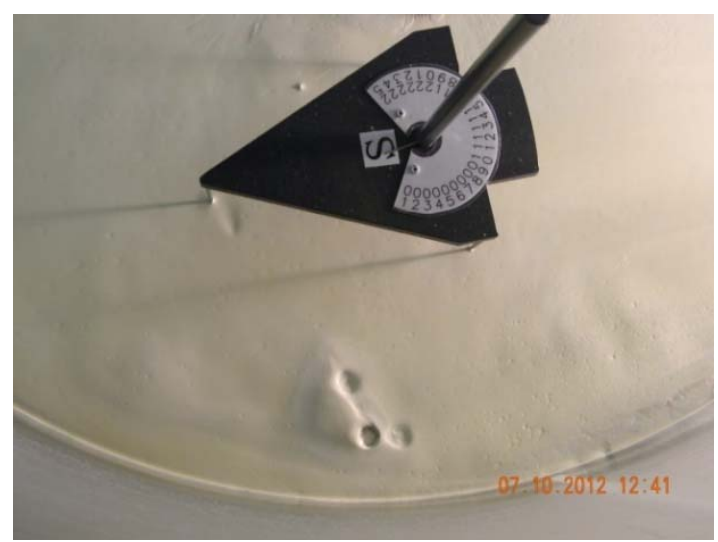

(a) 0.0 inch tank level

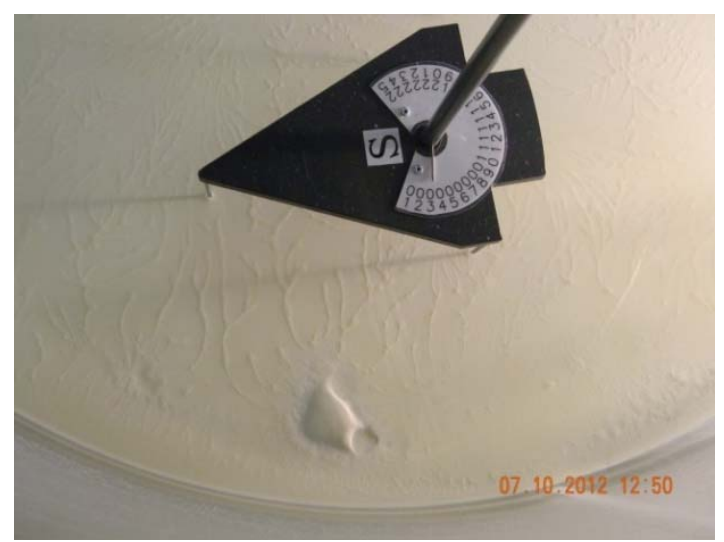

(b) 0.3 inch tank level

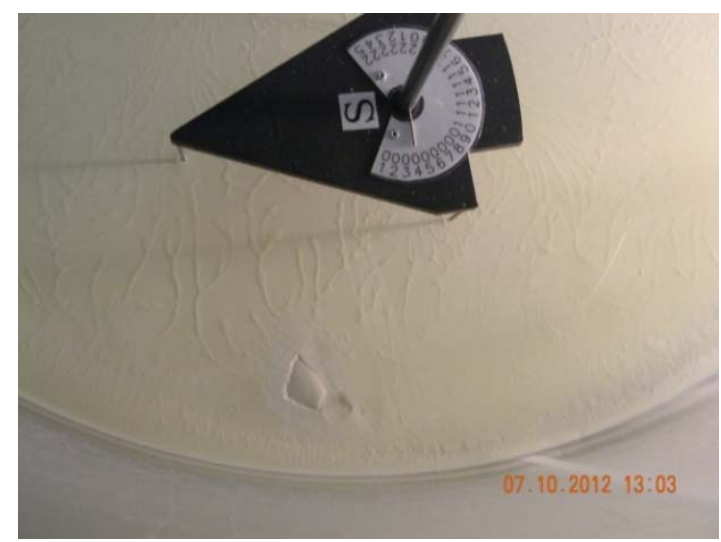

(c) 0.4 inch tank level

Figure 4-60. Photographs of changing tank level to estimate South mound volume: (a) to (c) 


\subsection{Comparison of Volume Estimates: Laser versus Photographic Techniques}

Table 4-5 shows the results of the volumes estimated of the North and South mounds after cycles 1,5 and 10 of both campaigns, which were graphically shown individually in Figure 4-19 and Figure 4-56. Having two ways to determine volumes is useful to confirm the measurements of each because of their respective measurement uncertainties, estimated to be: $\pm 5 \%$ for the laser method, and $\pm 20 \%$ of the photographic method, or a possible uncertainty $\pm 25 \%$ between methods. For the North (larger) mound the differences in the two methods ranged from $2 \%$ to $39 \%$ with an average difference of $22 \%$; therefore, the estimate uncertainty seems reasonable. However, for the South (smaller) mound the differences in the two methods ranged from $20 \%$ to $202 \%$ with an average of $83 \%$, well beyond the $20 \%$, which was not unexpected as stated in the last sentence of Section 3.8.2. These results show the limitation of photographic technique with small mounds and poorly contrasting surfaces.

The results do indicate the mound masses that can be expected for the solids concentrations used and mixing environment that existed. It is also interesting to note that the North mound was almost three to four times larger in volume than the South mound, which will be discussed later.

Table 4-5. Mound volumes of accumulated solids from both measurement techniques

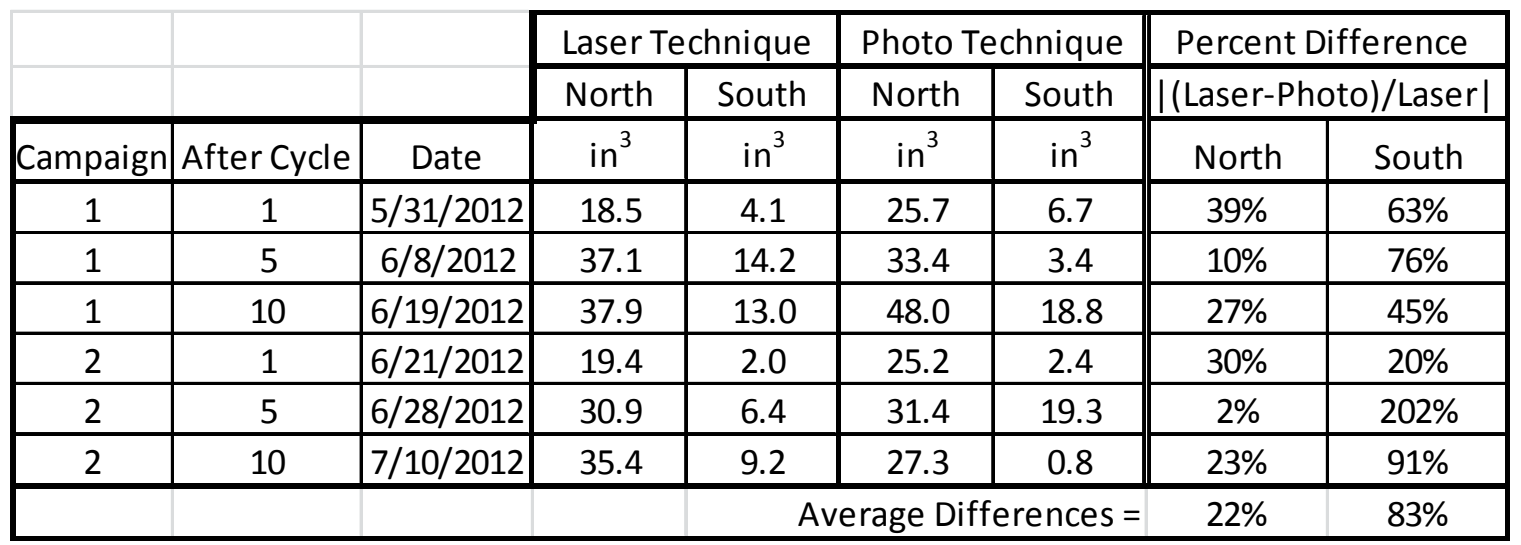




\subsubsection{Core Samples}

Another measure of the accumulated solids was the core samples and the method was described in Section 3.9. During the heel measurement sequence, and after the laser scan of the two mounds of accumulated solids was complete, several plugs (or cores, which is used interchangeably) of material was taken from the mounds to estimate the spacial location of the stainless steel particles. After Cycles 1 and 5 of each campaign core samples were taken from only one, and the same, mound so that the other mound would remain undisturbed until the final cycle. For the last cycle, Cycle 10 of each campaign, both mounds were sampled. The rationale was to compare the Cycle 10 North and South mound results to show if the act of sampling affected the results, but as was seen in the preceding section, Section 4.3.2.1.3, the South mound was 3 to 4 times smaller than the North mound making a comparison of those two mounds impossible. The decision to not sample one mound until the last cycle was made early in the task and before there was any knowledge of the mound or core sample sizes. The actual size of the samples was small. Figure 4-61 shows one sample taken during Campaign 2 and Figure 4-62 shows a Campaign 1 sample. As seen in the tabular data, Table 4-6 and Table 4-7 the total stainless steel (SS) mass in the core samples was less than 7 grams in each campaign and as will be seen the next section, this mass was less than $3 \%$ of the SS accumulated in the MDT.

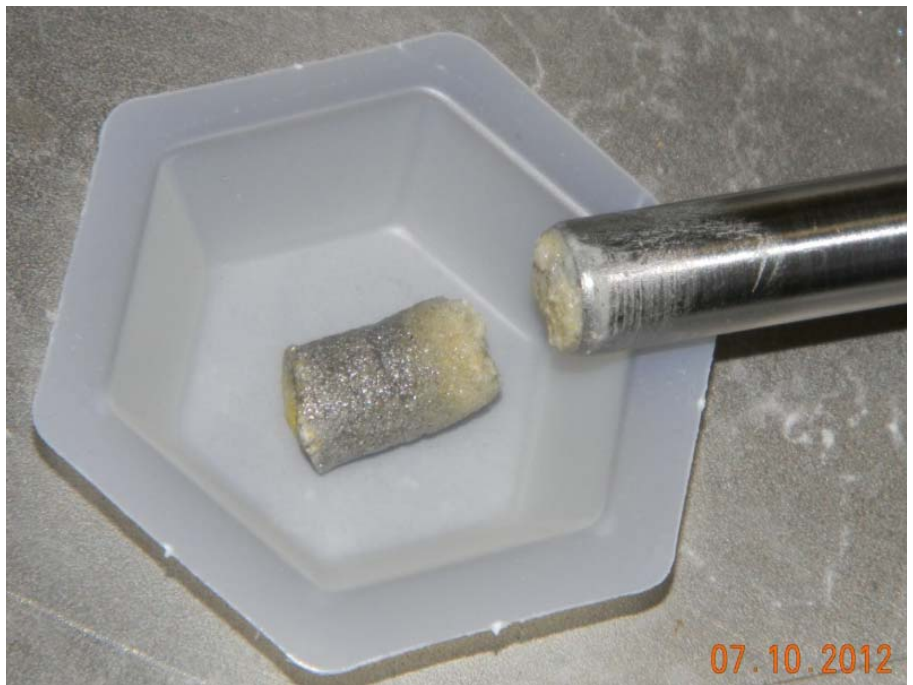

Figure 4-61. Campaign 2, core sample 20-2 taken from the North mound,

after Cycle 10, Table 4-7. It clearly shows the higher concentration of stainless steel particles at the bottom, which is the section furthest away from the end of the core sampler shown. The plug was $31 \mathrm{wt} \%$ in stainless steel, with the top portion having $4.8 \mathrm{wt} \%$ and the bottom having $46.1 \mathrm{wt} \%$. This plug length was estimated to be 0.56 -inch long from the laser measurement, which seems reasonable because the core sampler outside diameter was 3/8inch so the plug looks to be approximately $1 / 2$-inch long. Plugs of this size were generally cut into two pieces to be measured separately for stainless steel concentrations. 


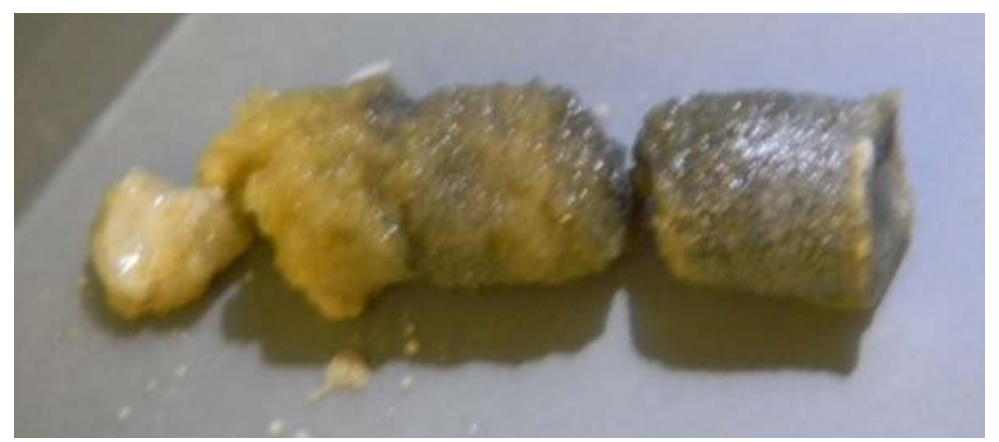

Figure 4-62. Campaign 1 core sample 5-6, Table 4-6, taken from the North mound, after Cycle 5 with the bottom of the plug to the right. The entire plug was $64 \%$ SS by weight. This core is shown right after it was extruded from the sampler. The split shown ocurred while the core was removed and the top portion was split again so a total of three segments could be measured. From the laser measurement of the mound this plug length was estimated to be 0.95 -inch long.

Table 4-6 shows the Campaign 1 results from the 11 samples taken and Table 4-7 shows the Campaign 2 results of the 11 samples taken, both in chronological order with the date the samples were taken listed in the first columns. In an attempt to quantify the vertical distribution of SS solids in the mounds samples were split into two or three segments when a plug was long enough; therefore, there are many more than just 11 rows in each table. The number of samples was not planned and the fact that both campaigns had the same quantity of samples same is a coincidence. The number and location of samples were decided upon during sampling because it was important to see the size, shape, and location of the mounds to take samples. Some of the criteria used to choose a sample location were: height of mound to obtain a substantial plug, evenly spaced samples along a long axis to determine trends, thin outer-edge sections to determine if SS is primarily locate at the edge of mounds.

The plug naming convention used in the two tables is as follows: In Table 4-6 the second column is $\mathrm{X}-\mathrm{Y}$ with $\mathrm{X}$ the cycle number and $\mathrm{Y}$ the plug number. In Table 4-7 the second column is $\mathrm{X}-\mathrm{Y}$ with $\mathrm{X}$ the cycle number +10 and $\mathrm{Y}$ the plug number, where the +10 is to more easily distinguish between the results of the two campaigns. Following the $\mathrm{Y}$ the symbols mean: $\mathrm{T}$ (=top), $\mathrm{M}$ (=middle), or B (=bottom), or no symbol (=single sample). That is, when possible the core samples were split to try to give a vertical concentration of SS in the accumulated solids.

The other features of the tables are:

1. Plug Height $=$ The estimated height based on the laser measured height at the sample location. Because the core sampler had to slightly compress the samples to remove a core in one piece then force changed the height slightly. Furthermore, when the plug was removed from the sample it became slightly longer or broke into pieces. The height given is for an entire plug and not for individual segments.

2. Mound Location $=$ North or South side of the MDT.

3. Filter grams $=$ The dry weight of the filter used to separate either the stainless steel or the other remaining solids.

4. SS (or Non-SS) and Filter grams $=$ The dry weight of the filtered solids and filter used.

5. Dried SS (or Non-SS) grams $=$ The dry weight of the solids without the filter

6. Percent SS in Plug = Dried SS mass / (Dried SS mass + Dried Non SS mass): Individual Segment. 
7. Percent SS in Full Plug = Dried SS mass / (Dried SS mass + Dried Non SS mass): All Segments of Plug.

Table 4-6. Campaign 1 core sample stainless steel concentrations

\begin{tabular}{|c|c|c|c|c|c|c|c|c|c|c|c|c|}
\hline & \multicolumn{11}{|c|}{ Core Sample Measurements for Campaign 1 after SS was separated from other solids } & \multirow[b]{2}{*}{ Percent } \\
\hline & & & Plug & & & SS & Dried & & Non-SS & Dried & Percent & \\
\hline Sample & Plug & Single or & Height & Mound & Filter & and Filter & SS & Filter & and Filter & Non-SS & SS in & SS in Full \\
\hline Date & Number & Partial Plug & Inches & Location & grams & grams & grams & grams & grams & grams & Plug, \% & Plug, \% \\
\hline $5 / 31 / 2012$ & $1-1$ & Single & 0.51 & North & 1.246 & 1.530 & 0.284 & 1.278 & 2.332 & 1.054 & $21.2 \%$ & $21.2 \%$ \\
\hline $5 / 31 / 2012$ & $1-2$ & Single & 0.25 & North & 1.268 & 1.272 & 0.004 & 1.227 & 1.810 & 0.583 & $0.7 \%$ & $0.7 \%$ \\
\hline $5 / 31 / 2012$ & $1-3$ & Single & (3) & North & 1.250 & 1.252 & 0.002 & 1.262 & 1.392 & 0.130 & $1.5 \%$ & $1.5 \%$ \\
\hline $5 / 31 / 2012$ & $1-4$ & Single & 0.24 & North & 1.219 & 1.469 & 0.250 & 1.217 & 2.070 & 0.853 & $22.7 \%$ & $22.7 \%$ \\
\hline $6 / 8 / 2012$ & $5-1$ & Single & 0.4 & North & 1.245 & 1.345 & 0.100 & .266 & 295 & 1.029 & $8.9 \%$ & $8.9 \%$ \\
\hline $6 / 8 / 2012$ & $5-2 T$ & Top & & North & 1.255 & 1.296 & 0.041 & 1.272 & .816 & 0.544 & $7.0 \%$ & \\
\hline $6 / 8 / 2012$ & $5-2 B$ & Bottom & 0.57 & North & 1.272 & 1.392 & 0.120 & 1.234 & 1.902 & 0.668 & $15.2 \%$ & $8.2 \%$ \\
\hline $6 / 8 / 2012$ & $5-3$ & Single & 0.33 & North & 1.243 & 1.533 & 0.290 & 1.247 & 208 & 0.961 & $23.2 \%$ & $23.2 \%$ \\
\hline $6 / 8 / 2012$ & $5-4$ & Single & 0.35 & North & 1.250 & 1.253 & 0.003 & 242 & 136 & 0.894 & $0.3 \%$ & $0.3 \%$ \\
\hline $6 / 8 / 2012$ & $5-5$ & Single & 0.57 & North & 1.221 & 2.438 & 1.217 & 1.293 & 794 & 1.501 & $44.8 \%$ & $44.8 \%$ \\
\hline $6 / 8 / 2012$ & $5-6 T$ & Top & & North & 1.270 & 1.267 & 0.000 & 1.258 & 1.940 & 0.682 & $0.0 \%$ & \\
\hline $6 / 8 / 2012$ & $5-6 \mathrm{M}$ & Middle & & North & 1.225 & 1.331 & 0.106 & 1.239 & 1.904 & 0.665 & $13.7 \%$ & \\
\hline $6 / 8 / 2012$ & $5-6 B$ & Bottom & 0.95 & North & 1.278 & 1.863 & 0.585 & 1.253 & 1.831 & 0.578 & $50.3 \%$ & $26.4 \%$ \\
\hline $6 / 19 / 2012$ & $10-1 T$ & & & North & 1.184 & 1.199 & 0.015 & 205 & 957 & 0.752 & $2.0 \%$ & \\
\hline $6 / 19 / 2012$ & $10-1 B$ & Bottom & 0.61 & North & 1.165 & 1.546 & 0.381 & 139 & 813 & 0.674 & $36.1 \%$ & $21.7 \%$ \\
\hline $6 / 19 / 2012$ & $10-2 \mathrm{~T}$ & Top & & North & 1.194 & 1.187 & 0.000 & 1.178 & 1.743 & 0.565 & $0.0 \%$ & \\
\hline $6 / 19 / 2012$ & $10-2 B(1)$ & Bottom & 0.39 & North & 1.136 & 1.138 & 0.002 & 1.152 & 1.617 & 0.465 & $0.4 \%$ & $0.2 \%$ \\
\hline $6 / 19 / 2012$ & $10-3 \mathrm{~T}$ & Top & & North & 1.162 & 1.294 & 0.132 & 1.192 & 170 & 0.978 & $11.9 \%$ & \\
\hline $6 / 19 / 2012$ & $10-3 B$ & Bottom & 0.76 & North & 1.150 & 2.067 & 0.917 & 186 & 817 & 0.631 & $59.2 \%$ & $39.5 \%$ \\
\hline $6 / 19 / 2012$ & $10-4 \mathrm{~T}$ & Top & & North & 1.196 & 235 & 0.039 & 187 & 955 & 0.768 & $4.8 \%$ & \\
\hline $6 / 19 / 2012$ & $10-4 B$ & Bottom & 0.54 & North & 1.183 & 1.338 & 0.155 & 1.180 & 1.992 & 0.812 & $16.0 \%$ & $10.9 \%$ \\
\hline $6 / 19 / 2012$ & $10-5 T$ & Top & & North & 1.197 & 1.366 & 0.169 & 1.199 & 2.053 & 0.854 & $16.5 \%$ & \\
\hline $6 / 19 / 2012$ & $10-5 B$ & Bottom & 0.63 & North & 1.215 & 1.470 & 0.255 & 1.196 & 1.912 & 0.716 & $26.3 \%$ & $21.3 \%$ \\
\hline $6 / 19 / 2012$ & $10-6 \mathrm{~T}$ & Top & & North & 1.193 & & 0.017 & 1.201 & 718 & 0.517 & $3.2 \%$ & \\
\hline $6 / 19 / 2012$ & $10-6 B$ & Bottom & 0.43 & North & 1.196 & 1.181 & 0.000 & 1.197 & 1.709 & 0.512 & $0.0 \%$ & $1.6 \%$ \\
\hline $6 / 19 / 2012$ & $10-7 T$ & Top & & South & 1.166 & 1.187 & 0.021 & 1.224 & 2.003 & 0.779 & $2.6 \%$ & \\
\hline $6 / 19 / 2012$ & $10-7 \mathrm{~B}$ & Bottom & 0.29 & South & 1.204 & 1.153 & 0.000 & 1.206 & 1.127 & 0.000 & $0.0 \%$ & $2.6 \%$ \\
\hline $6 / 19 / 2012$ & $10-8 \mathrm{~T}(2)$ & Top & & South & 1.202 & 1.982 & 0.780 & 1.194 & 1.605 & 0.411 & $65.5 \%$ & \\
\hline $6 / 19 / 2012$ & $10-8 \mathrm{~B}(2)$ & Bottom & 0.24 & South & 1.175 & 1.367 & 0.192 & 1.200 & 1.666 & 0.466 & $29.2 \%$ & $52.6 \%$ \\
\hline $6 / 19 / 2012$ & $10-9$ & Single & 0.28 & South & 1.205 & 1.502 & 0.297 & 1.239 & 2.089 & 0.850 & $25.9 \%$ & $25.9 \%$ \\
\hline $6 / 19 / 2012$ & $10-10 \mathrm{~T}$ & Top & & South & 1.232 & 1.225 & 0.000 & 1.205 & 1.920 & 0.715 & $0.0 \%$ & \\
\hline $6 / 19 / 2012$ & $10-10 B$ & Bottom & 0.48 & South & 1.207 & 1.269 & 0.062 & 1.191 & 1.875 & 0.684 & $8.3 \%$ & $4.2 \%$ \\
\hline $6 / 19 / 2012$ & $10-11 T$ & Top & & South & 1.181 & 1.227 & 0.046 & 1.231 & 1.964 & 0.733 & $5.9 \%$ & \\
\hline $6 / 19 / 2012$ & \begin{tabular}{|l|}
$10-11 B$ \\
\end{tabular} & Bottom & 0.4 & South & 1.197 & 1.445 & 0.248 & 1.175 & 1.683 & 0.508 & $32.8 \%$ & $19.2 \%$ \\
\hline \multicolumn{5}{|c|}{ (1) Non-SS filter had hole affecting measurement } & \multicolumn{2}{|c|}{ Total SS in Cores $=$} & 6.7 & grams & & & & \\
\hline \multicolumn{5}{|c|}{ (2) Top and Bottom sample may have been switched } & & & & & & & & \\
\hline \multicolumn{13}{|c|}{ (3) Plug was taken at a location near the center of the tank where the lasers could not scan. The height is estimated at $1 / 8$ th inch. } \\
\hline
\end{tabular}

One plug, i.e., 1-3, shown in Table 4-6, does not show a plug height (the fourth column). Plug 13 was taken very close to the tank center to determine if there was SS in the $\sim 1 / 8$ inch layer of material the coated the entire tank bottom. The plug contained an insignificant amount of SS, i.e., 0.002 grams, and it's height probably was close to $1 / 8$ inch. 
Table 4-7. Campaign 2 core sample stainless steel concentrations

\begin{tabular}{|c|c|c|c|c|c|c|c|c|c|c|c|c|}
\hline & Core Sar & nple Meas & ureme & ts for $\mathrm{Ca}$ & mpaign & 2 after $S$ & $S$ was & parate & d from ot & her sol & & \\
\hline & & & Plug & & & SS & Dried & & Non-SS & Dried & Percent & Percent \\
\hline Sample & Plug & Single or & Height & Mound & Filter & and Filter & SS & Filter & and Filter & Non-SS & SS in & SS in Full \\
\hline Date & Number & Partial Plug & Inches & Location & grams & grams & grams & grams & grams & grams & Plug, \% & Plug, \% \\
\hline $6 / 21 / 2012$ & $11-1 \mathrm{~T}$ & \begin{tabular}{|l|} 
Top \\
\end{tabular} & & \begin{tabular}{|l|} 
North \\
\end{tabular} & 0.624 & 0.693 & 0.069 & 0.642 & 1.369 & 0.727 & $8.7 \%$ & \\
\hline $6 / 21 / 2012$ & 11-1B & Bottom & 0.48 & North & 0.647 & 0.897 & 0.250 & 0.648 & 1.184 & 0.536 & $31.8 \%$ & $20.2 \%$ \\
\hline $6 / 21 / 2012$ & $11-2 \mathrm{~T}$ & Top & & North & 0.626 & 0.697 & 0.071 & 0.643 & 1.386 & 0.743 & $8.7 \%$ & \\
\hline $6 / 21 / 2012$ & 11-2B & Bottom & 0.53 & North & 0.629 & 1.024 & 0.395 & 0.668 & 1.311 & 0.643 & $38.1 \%$ & $25.2 \%$ \\
\hline $6 / 21 / 2012$ & 11-3T & Top & & North & 0.665 & 0.694 & 0.029 & 0.635 & 1.698 & 1.063 & $2.7 \%$ & \\
\hline $6 / 21 / 2012$ & 11-3B & Bottom & 0.54 & North & 0.642 & 1.029 & 0.387 & 0.644 & 1.202 & 0.558 & $41.0 \%$ & $20.4 \%$ \\
\hline $6 / 21 / 2012$ & $11-4 \mathrm{~T}$ & Top & & North & 0.643 & 0.685 & 0.042 & 0.633 & 1.355 & 0.722 & $5.5 \%$ & \\
\hline $6 / 21 / 2012$ & 11-4B & Bottom & 0.47 & North & 0.633 & 0.801 & 0.168 & 0.642 & 1.207 & 0.565 & $22.9 \%$ & $14.4 \%$ \\
\hline $6 / 21 / 2012$ & 11-5T & Top & & North & 0.640 & 0.782 & 0.142 & 0.634 & 1.298 & 0.664 & $17.6 \%$ & \\
\hline $6 / 21 / 2012$ & 11-5B & Bottom & 0.55 & North & 0.653 & 0.765 & 0.112 & 0.650 & 1.277 & 0.627 & $15.2 \%$ & $16.4 \%$ \\
\hline $6 / 21 / 2012$ & $11-6 \mathrm{~T}$ & Top & & North & 0.648 & 0.657 & 0.009 & 0.628 & 1.154 & 0.526 & $1.7 \%$ & \\
\hline $6 / 21 / 2012$ & 11-6B & Bottom & 0.33 & North & 0.621 & 0.715 & 0.094 & 0.640 & 1.104 & 0.464 & $16.8 \%$ & $9.4 \%$ \\
\hline $6 / 28 / 2012$ & $15-1 T$ & Top & & North & 0.584 & 0.834 & 0.250 & 0.620 & 1.371 & 0.751 & $25.0 \%$ & \\
\hline $6 / 28 / 2012$ & $15-1 B$ & Bottom & 0.57 & North & 0.607 & 0.848 & 0.241 & 0.638 & .065 & 0.427 & $36.1 \%$ & $29.4 \%$ \\
\hline $6 / 28 / 2012$ & $15-2 \mathrm{~T}$ & Top & & North & 0.620 & 0.687 & 0.067 & 0.655 & 1.106 & 0.451 & $12.9 \%$ & \\
\hline $6 / 28 / 2012$ & $15-2 M$ & Middle & & North & 0.603 & 1.166 & 0.563 & 0.587 & 1.079 & 0.492 & $53.4 \%$ & \\
\hline $6 / 28 / 2012$ & $15-2 B$ & Bottom & 0.62 & North & 0.605 & 1.329 & 0.724 & 0.592 & 0.902 & 0.310 & $70.0 \%$ & $51.9 \%$ \\
\hline $6 / 28 / 2012$ & 15-3T & Top & & North & 0.587 & 0.601 & 0.014 & 0.589 & 1.104 & 0.515 & $2.6 \%$ & \\
\hline $6 / 28 / 2012$ & 15-3B & Bottom & 0.36 & North & 0.578 & 0.707 & 0.129 & 0.589 & 0.996 & 0.407 & $24.1 \%$ & $13.4 \%$ \\
\hline $6 / 28 / 2012$ & $15-4 \mathrm{~T}$ & Top & & North & 0.608 & 0.706 & 0.098 & 0.607 & 1.379 & 0.772 & $11.3 \%$ & \\
\hline $6 / 28 / 2012$ & $15-4 \mathrm{~B}$ & Bottom & 0.50 & North & 0.595 & 0.698 & 0.103 & 0.597 & 1.099 & 0.502 & $17.0 \%$ & $13.6 \%$ \\
\hline $6 / 28 / 2012$ & $15-5 \mathrm{~T}$ & Top & & North & 0.613 & 0.679 & 0.066 & 0.602 & 180 & 0.578 & $10.2 \%$ & \\
\hline $6 / 28 / 2012$ & $15-5 B$ & Bottom & 0.42 & North & 0.593 & 0.634 & 0.041 & 0.604 & 1.188 & 0.584 & $6.6 \%$ & $8.4 \%$ \\
\hline $6 / 28 / 2012$ & $15-6$ & Single & 0.30 & North & 0.606 & 0.631 & 0.025 & 0.598 & 1.274 & 0.676 & $3.6 \%$ & $3.6 \%$ \\
\hline $6 / 28 / 2012$ & $15-7 T$ & Top & & North & 0.592 & 0.600 & 0.008 & 0.588 & 1.063 & 0.475 & $1.7 \%$ & \\
\hline $6 / 28 / 2012$ & 15-7B & Bottom & 0.37 & North & 0.598 & 0.603 & 0.005 & 0.608 & 0.961 & 0.353 & $1.4 \%$ & $1.5 \%$ \\
\hline $7 / 10 / 2012$ & $20-1 T$ & Top & & North & 0.564 & 0.693 & 0.129 & 0.606 & 1.462 & 0.856 & $13.1 \%$ & \\
\hline $7 / 10 / 2012$ & $20-1 B$ & Bottom & 0.69 & North & 0.597 & 1.395 & 0.798 & 0.595 & 1.270 & 0.675 & $54.2 \%$ & $37.7 \%$ \\
\hline $7 / 10 / 2012$ & $20-2 T$ & Top & & North & 0.602 & 0.632 & 0.030 & 0.600 & 1.193 & 0.593 & $4.8 \%$ & \\
\hline $7 / 10 / 2012$ & $20-2 B$ & Bottom & 0.56 & North & 0.611 & 1.106 & 0.495 & 0.604 & 1.182 & 0.578 & $46.1 \%$ & $31.0 \%$ \\
\hline $7 / 10 / 2012$ & $20-3 T$ & Top & & North & 0.602 & 0.752 & 0.150 & 0.612 & 1.213 & 0.601 & $20.0 \%$ & \\
\hline $7 / 10 / 2012$ & $20-3 B$ & Bottom & 0.38 & North & 0.600 & 1.139 & 0.539 & 0.585 & 0.883 & 0.298 & $64.4 \%$ & $43.4 \%$ \\
\hline $7 / 10 / 2012$ & $20-4$ & Single & 0.28 & North & 0.596 & 0.670 & 0.074 & 0.598 & 1.282 & 0.684 & $9.8 \%$ & $9.8 \%$ \\
\hline $7 / 10 / 2012$ & $20-5 T$ & Top & & North & 0.606 & 0.617 & 0.011 & 0.599 & 1.235 & 0.636 & $1.7 \%$ & \\
\hline $7 / 10 / 2012$ & $20-5 B$ & Bottom & 0.55 & North & 0.601 & 0.710 & 0.109 & 0.588 & 1.303 & 0.715 & $13.2 \%$ & $8.2 \%$ \\
\hline $7 / 10 / 2012$ & $20-6 \mathrm{~T}$ & Top & & North & 0.598 & 0.625 & 0.027 & 0.598 & 0.899 & 0.301 & $8.2 \%$ & \\
\hline $7 / 10 / 2012$ & $20-6 B$ & Bottom & 0.51 & North & 0.599 & 0.687 & 0.088 & 0.567 & 1.393 & 0.826 & $9.6 \%$ & $9.3 \%$ \\
\hline \begin{tabular}{|l|}
$7 / 10 / 2012$ \\
\end{tabular} & $20-7 T$ & Top & & North & 0.594 & 0.604 & 0.010 & 0.597 & 1.137 & 0.540 & $1.8 \%$ & \\
\hline \begin{tabular}{|l|}
$7 / 10 / 2012$ \\
\end{tabular} & $20-7 \mathrm{~B}$ & Bottom & 0.35 & North & 0.603 & 0.640 & 0.037 & 0.591 & 1.058 & 0.467 & $7.3 \%$ & $4.5 \%$ \\
\hline $7 / 10 / 2012$ & $20-8$ & Single & 0.22 & North & 0.583 & 0.743 & 0.160 & 0.608 & 1.243 & 0.635 & $20.1 \%$ & $20.1 \%$ \\
\hline $7 / 10 / 2012$ & $20-9$ & Single & 0.09 & South & 0.602 & 0.607 & 0.005 & 0.582 & 0.851 & 0.269 & $1.8 \%$ & $1.8 \%$ \\
\hline $7 / 10 / 2012$ & $20-10 \mathrm{~T}$ & Top & & South & 0.599 & 0.622 & 0.023 & 0.602 & 0.910 & 0.308 & $6.9 \%$ & \\
\hline $7 / 10 / 2012$ & $20-10 \mathrm{~B}$ & Bottom & 0.18 & South & 0.595 & 0.000 & 0.000 & 0.612 & 0.000 & 0.000 & $0.0 \%$ & $6.9 \%$ \\
\hline \multirow[t]{2}{*}{$7 / 10 / 2012$} & $20-11$ & Single & 0.13 & South & 0.596 & 0.607 & 0.011 & 0.600 & 1.058 & 0.458 & $2.3 \%$ & $2.3 \%$ \\
\hline & & & & & \multicolumn{2}{|c|}{ Total SS in Cores $=$} & 6.8 & grams & & & & \\
\hline
\end{tabular}


The following eight figures, Figure 4-63 to Figure 4-70, repeat a subset of the 3-D laser graphs previous shown, but now the locations of the core samples are indicated. The full information on each of the samples taken is give in Table 4-6 and Table 4-7, but for convenience the caption of each figure describes pertinent information to better understand the results. The captions include the following information:

- If it is the North or South mound

- Reference to related figures: The first is to a photograph of the specific mound and the second is to the laser graph without showing the core sample locations.

- The Cycle and Campaign during which the sample was taken.

- A list with the plug number, full plug height, and SS mass concentration of the full plug. Concentrations of individual segments of a plug are in Table 4-6 and Table 4-7.

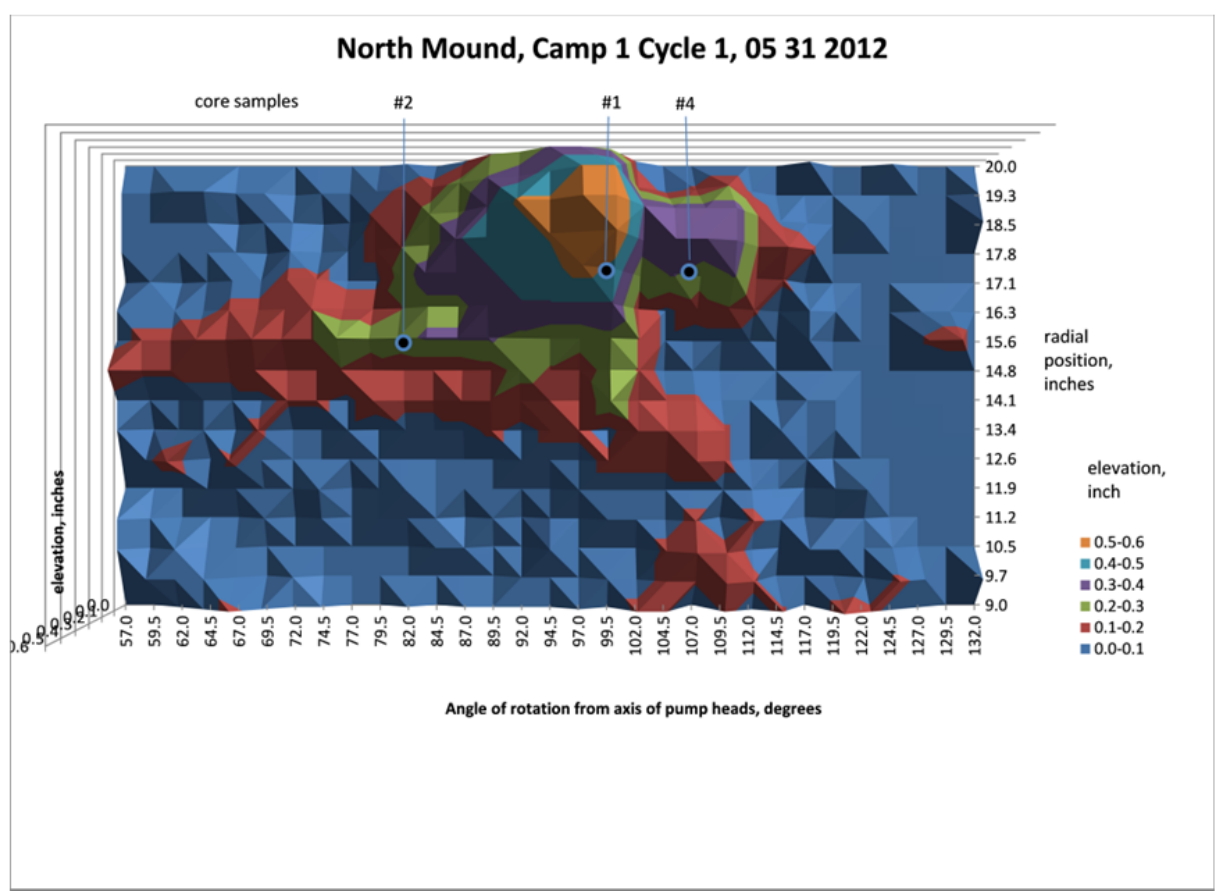

Figure 4-63. North mound top view after Cycle 1 of Campaign 1, compare to Figure 4-20 and Figure 4-23, with core sample locations. [Plug \#: height (SS\%) = 1: 0.51 in. (21.2\%); 2: 0.25 in. (0.7\%); 3:>0.13 in. (1.5\%); 4: 0.24 in. (22.7\%)] Note: Plug \#3 was out of range of scale. 


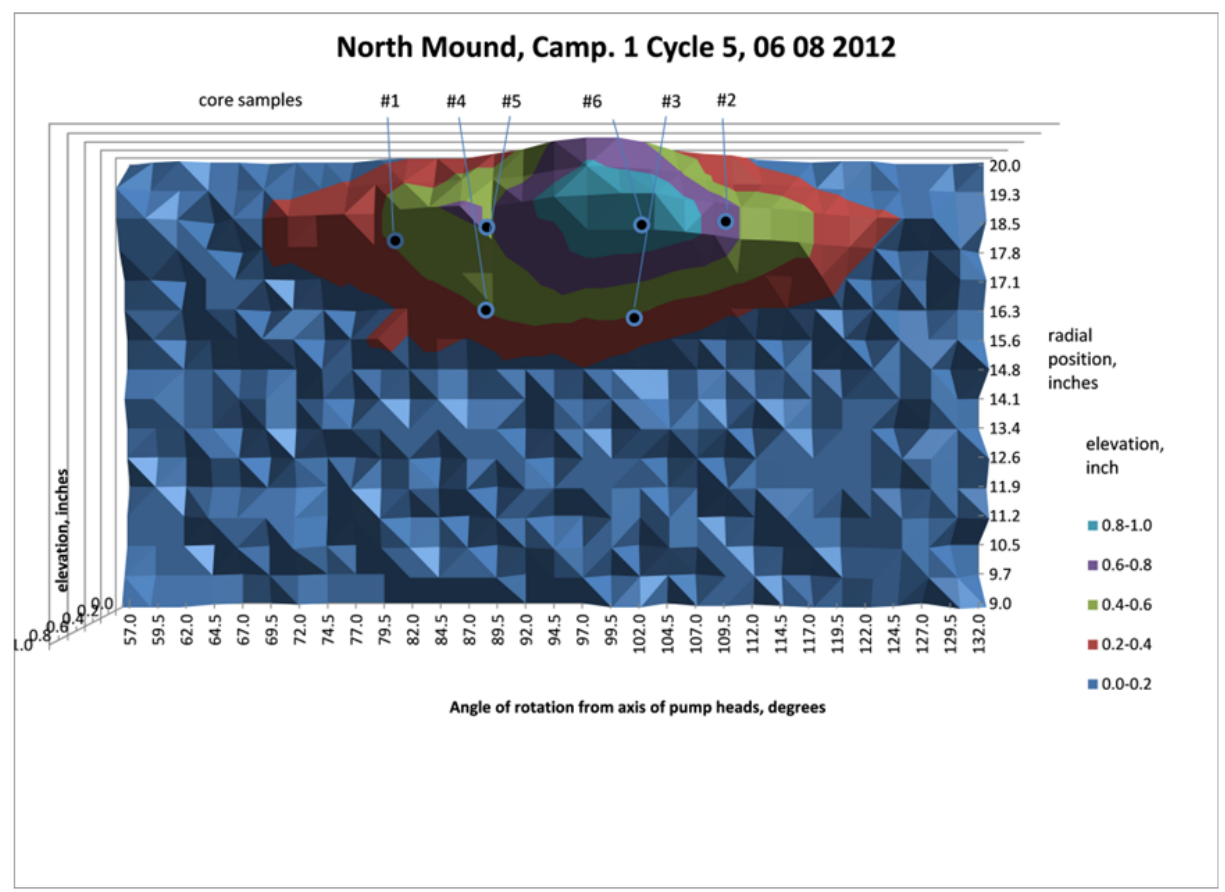

Figure 4-64. North mound top view after Cycle 5 of Campaign 1, compare to Figure 4-26 and Figure 4-29, 1 with core sample locations. [Plug \#: height $(S S \%)=1: 0.40$ in. (8.9\%); 2: 0.57 in. (8.2\%); 3: 0.33 in. (23.2\%); $4: 0.35$ in. (0.3\%); 5: 0.57 in. (44.8\%); 6: 0.95 in. (26.4\%)].

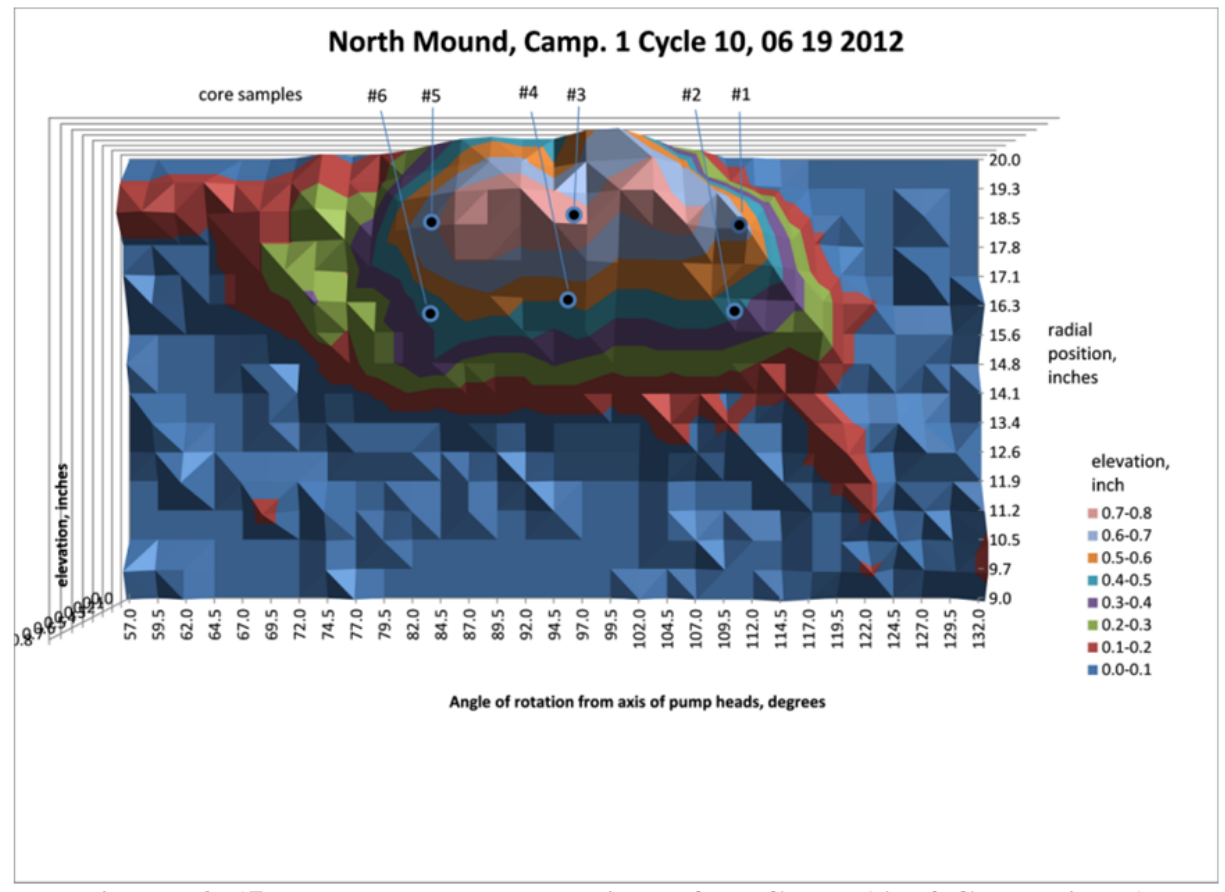

Figure 4-65. North mound top view after Cycle 10 of Campaign 1, compare to Figure 4-32 and Figure 4-35, with core sample locations. [Plug \#: height (SS\%) = 1: 0.61 in. (21.7\%); 2 : 0.39 in. (0.2\%); 3: 0.76 in. (39.5\%); 4: 0.54 in. (10.9\%); 5: 0.63 in. (21.3\%); 6: 0.43 in. (1.6\%)]. 


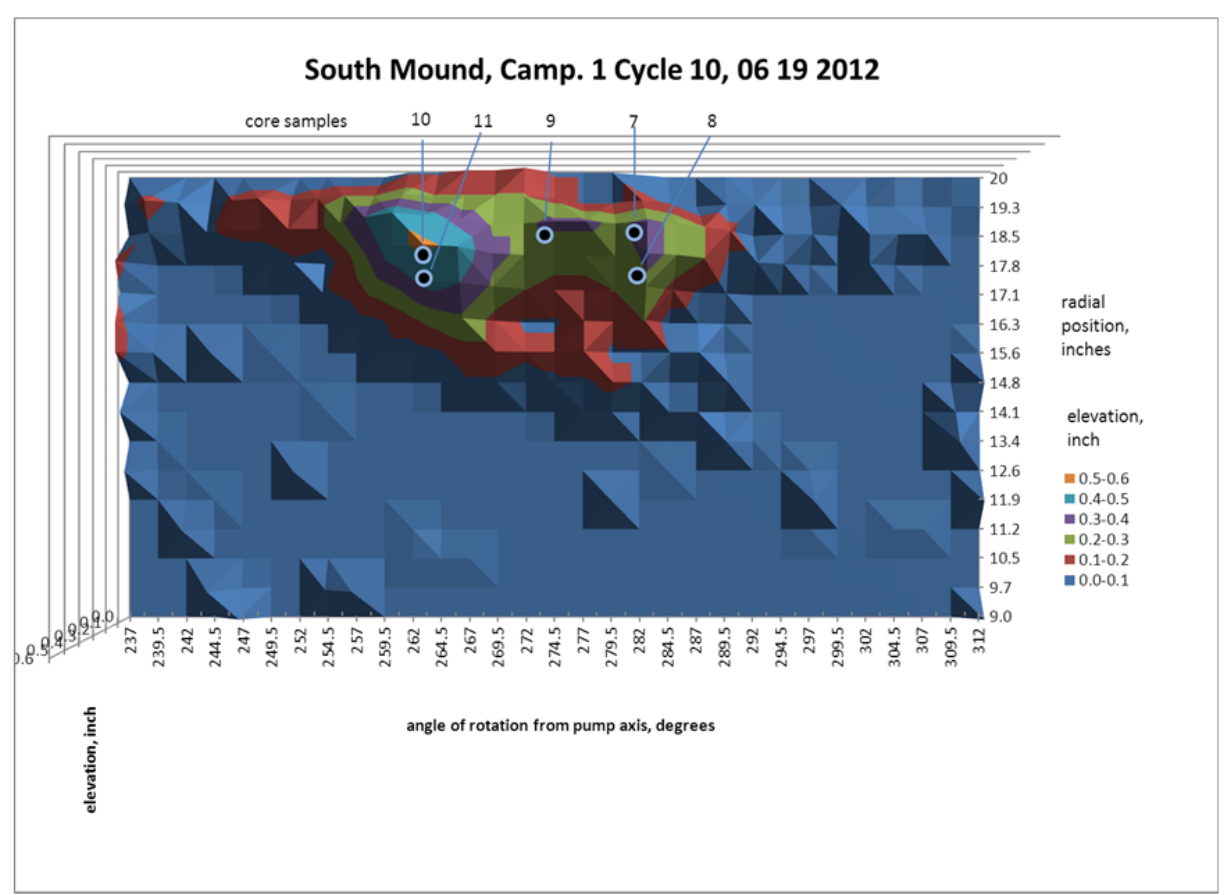

Figure 4-66. South mound top view after Cycle 10 of Campaign 1, compare to Figure 4-33 and Figure 4-37, with core sample locations. [Plug \#: height (SS\%) = 7: 0.29 in, (2.6\%); 8: 0.24 in. (52.6\%); 9: 0.28 in. (25.9\%; 10: 0.48 in. (4.2\%); 11: 0.4 in. $(\mathbf{1 9 . 2 \% ) ] . ~}$

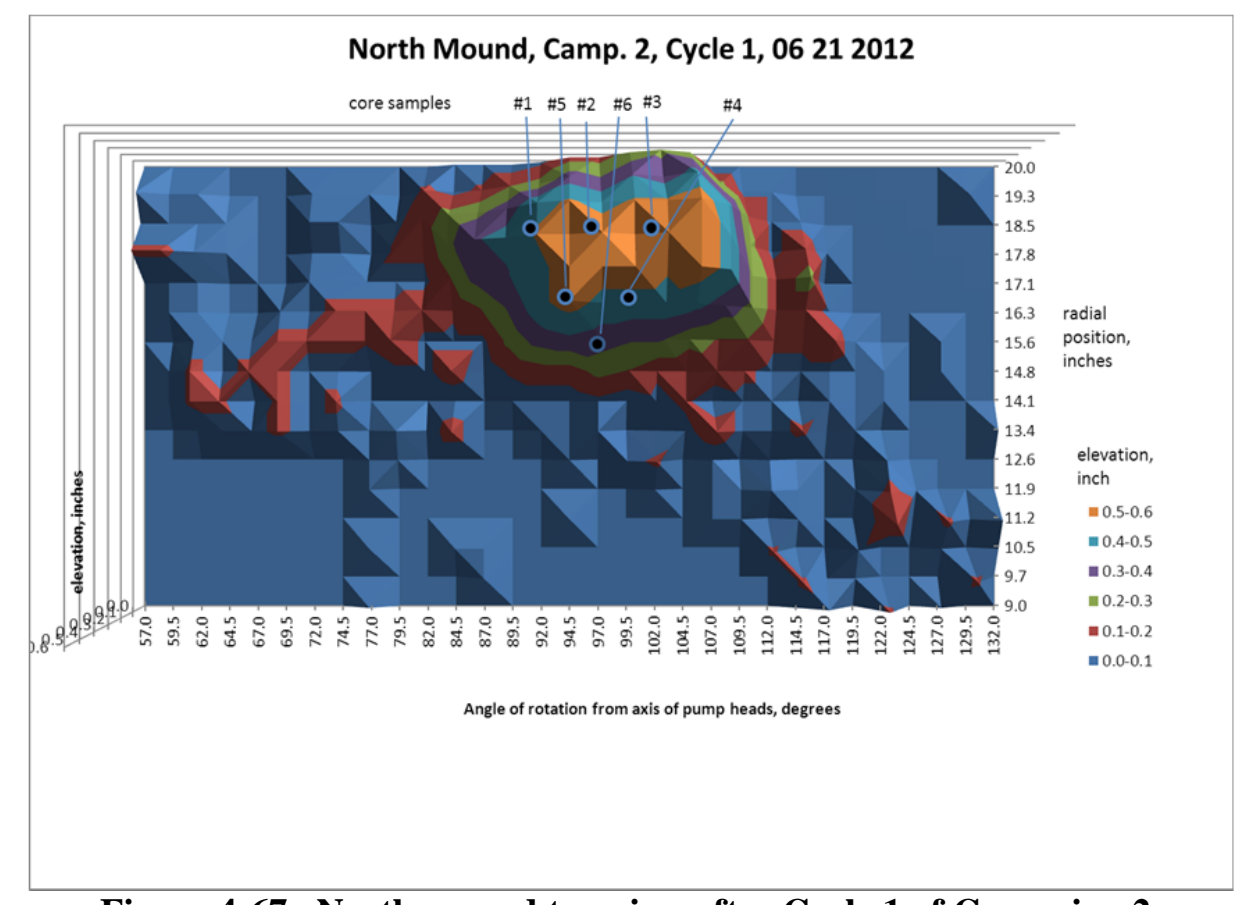

Figure 4-67. North mound top view after Cycle 1 of Campaign 2, compare to Figure 4-38 and Figure 4-41, with core sample locations. [Plug \#: height (SS\%) = 1: 0.48 in, (20.2\%); 2: 0.53 in. (25.2\%); $3: 0.54$ in. (20.4\%); $4: 0.47$ in. (14.4\%); $5: 0.55$ in. $(16.4 \%) ; 6$ : 0.33 in. $(9.4 \%)]$. 


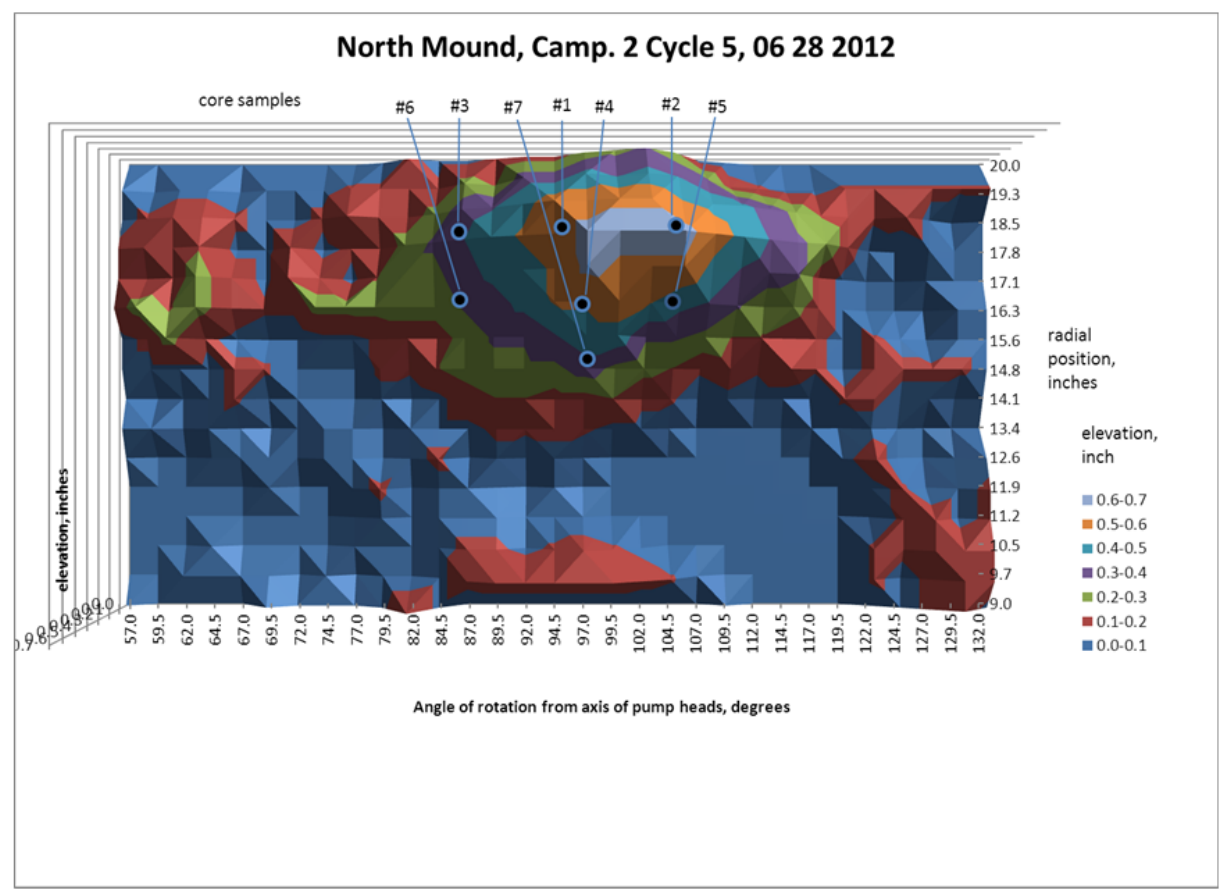

Figure 4-68. North mound top view after Cycle 5 of Campaign 2, compare to Figure 4-44 and Figure 4-47, with core sample locations. [Plug \#: height (SS\%) = 1: 0.57 in, (29.4\%); 2: 0.62 in. (51.9\%); $3: 0.36$ in. (13.4\%); 4: 0.50 in. (13.6\%); 5: 0.42 in.

(8.4\%); 6: 0.30 in. (3.6\%); 7: 0.37 in. (1.7\%)].

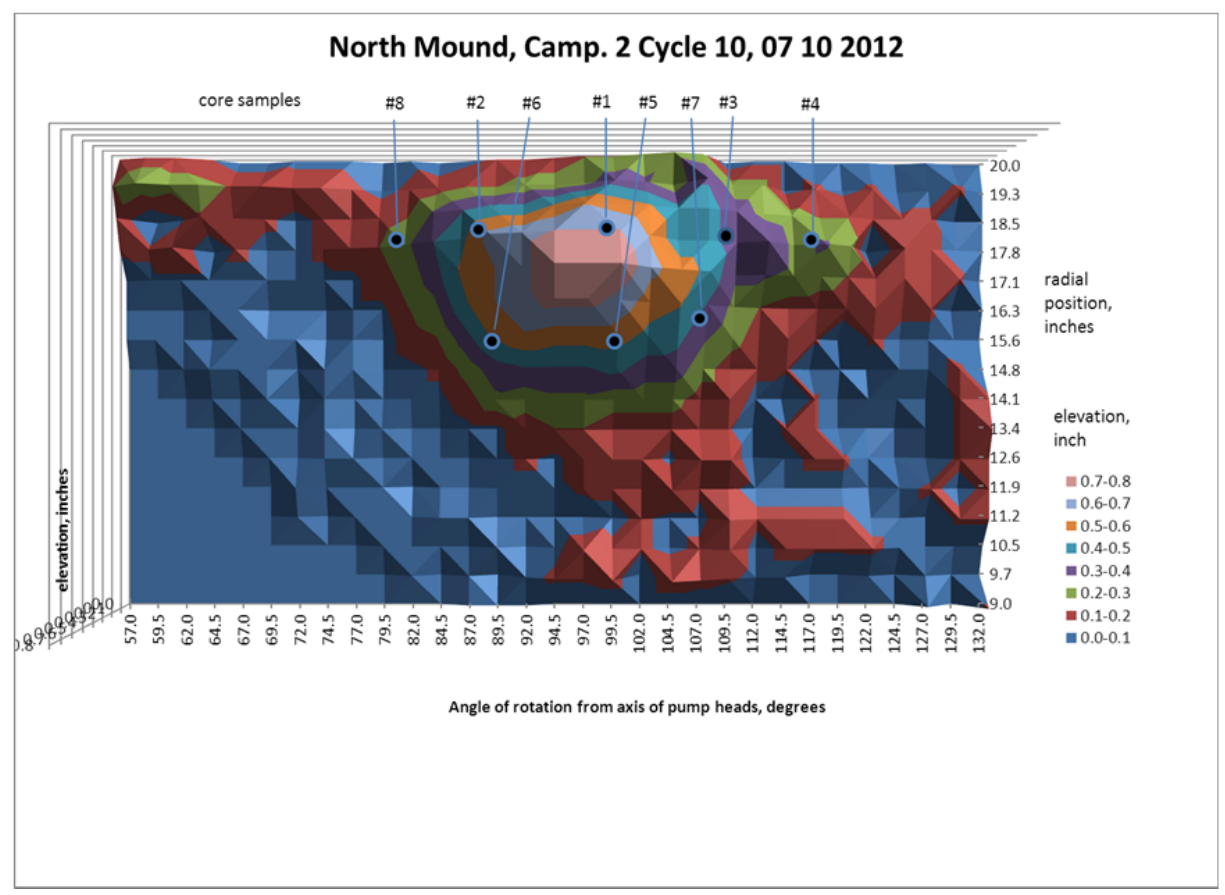

Figure 4-69. North mound top view after Cycle 10 of Campaign 2, compare to Figure 4-50 and Figure 4-53, with core sample locations. [Plug \#: height (SS\%) = 1: 0.69 in, (37.7\%); 2: 0.56 in. (31.0\%); 3: 0.38 in. (43.4\%); 4: 0.28 in. (9.8\%); 5: 0.55 in. (8.2\%); 6: 0.51 in. (9.3\%); 7: 0.35 in. (4.5\%); 8: 0.22 in. (1.8\%)]. 


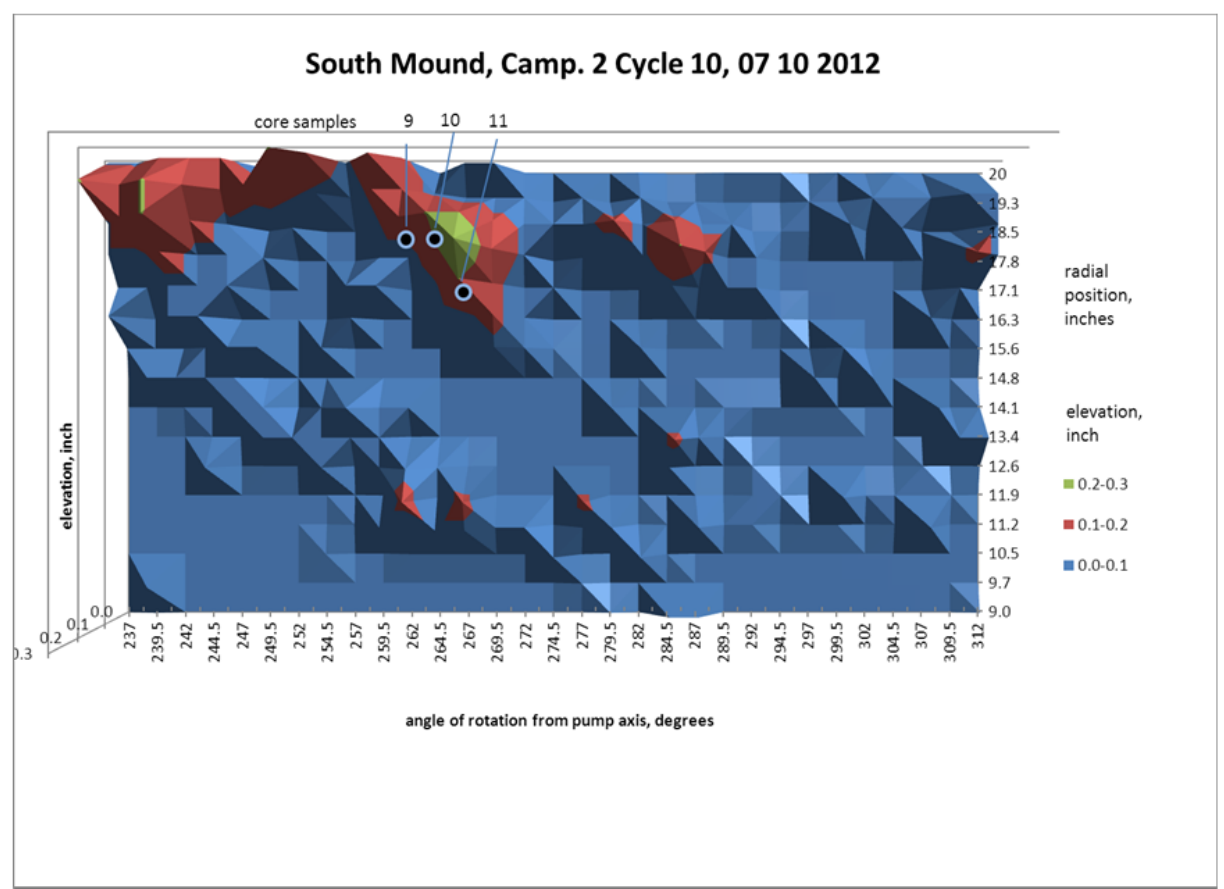

Figure 4-70. South mound top view after Cycle 10 of Campaign 2, compare to Figure 4-51 and Figure 4-55, with core sample locations. [Plug \#: height (SS\%) = 9: 0.09 in, $(1.8 \%)$; $10: 0.18$ in. $(6.9 \%)$; $11: 0.13$ in. $(2.3 \%)]$.

From the data tables and the figures the locations of the SS solids was not surprising. The segmented plugs showed that most of the SS resided in the bottom segment, for example, each campaign had one plug long enough to be divided into three segments: Table 4-6, plug No. 5-6 the segments had the follow concentrations of SS: top $0 \%$, middle $14 \%$, bottom $50 \%$. Table $4-7$, plug No. 15-2, the segments had the follow concentrations of SS: top $13 \%$, middle $54 \%$, bottom $70 \%$. Perusing all the other segmented plug shows the SS resided in the bottom half. There are a couple of exceptions, and as is noted in the tables it is believed that those segments were accidentally reversed.

With the information given in the sample locations on the laser graphs the general trend is that the SS concentration was the heaviest in the largest sections of the mound and basically followed along the radial dimension and balanced. A good example would be Figure 4-65, which shows the North mound in Cycle 10 of Campaign 1. Three evenly spaced core samples were at about 16 inches from the center and numbered 2, 4, and 6. Those samples had SS concentrations of $0.2 \%$, $11 \%$, and $2 \%$, respectively. Three core samples, with the samples even spaced, were taken at about 18 inches from the center and numbered 1,3, and 5. Those samples had SS concentrations of $22 \%, 40 \%$, and $21 \%$, respectively.

When this task began it was assumed that SS would build up around the edges of the mounds but that wasn't evident. Only a few samples were taken at the other edges of the mounds because it was hard to pick up enough material to measure, but a few were captured. For example, Plug No. 7 of Cycle 5 of Campaign 2 (North mound), Figure 4-68, only had a 2\% SS concentration; Plug No. 8 of Cycle 10 of Campaign 2 (North mound), Figure 4-69, only had 2\% SS concentration; 
Plugs 9 and 11 of Cycle 10 of Campaign 2 (South mound), Figure 4-70, only had 2\% SS concentration each. Clearly the SS resides closer to the center of the mounds.

\subsubsection{Accumulated Solids Captured after Cycle 10 of Each Campaign}

As mentioned in Section 3.10, at the end of each campaign and after Cycle 10 the accumulated stainless steel solids were collected from the MDT. This was done after the mounds of solids were measured for volume and the core samples removed. For Campaign 1 the heel material that was completely returned to the MDT after the photographic technique to measure mound was finished. The simulant was then mixed vigorously to suspend all the remaining MDT solids and then the heel was pumped through the magnetic separator to capture the stainless steel solids. Because all of the MDT simulant could not be pump out, the remaining stainless steel was captured with hand-held magnets. For Campaign 2, only the hand-held magnets were used because it was just as fast, if not faster, then using the two-step method of first using the in-line magnetic separator and then the hand-held separator. More importantly, the process used for Campaign 1 caused the loss of information as to the locations of the remaining stainless steel with respect to tank regions, i.e., in the mounds or spread around the tank bottom. Therefore, for Campaign 2 an effort was made to collect the stainless steel particles by region: North Mound, South Mound, and from All Other Areas, see Table 4-8. The selection of mound areas and "all other areas" was subjective. The two mounds were located opposite each other in the tank. When the settled solids transitioned from the general level plain that covered most of the tank bottom to a continuous increase in solids height then those elevated solids were probed for stainless steel to be classified as mound material. After both mounds were probed, then the rest of the tank was probed from the remaining stainless steel and classified as "all other areas." For both campaigns the separated material was then thoroughly washed and further separated with magnets to hopefully remove all other solids to obtain an accurate mass measure of the stainless steel that was left in the MDT after the end of each campaign. The masses of stainless steel measured were:

Table 4-8. Stainless steel recovered from Cycle 10 Heel after each campaign

\begin{tabular}{|c|c|c|c|}
\hline Campaign & Grams of SS & $\%$ Total & Location \\
\hline 1 & 291.5 & $100 \%$ & Total Heel \\
\hline \hline 2 & 182.9 & $75 \%$ & North Mound \\
\hline 2 & 44.1 & $18 \%$ & South Mound \\
\hline 2 & 17.5 & $7 \%$ & All Other Areas \\
\hline \hline 2 & 244.5 & $100 \%$ & Total Heel \\
\hline
\end{tabular}

Table 4-8 shows that more SS remained in the MDT after all cycles of Campaign 1 than Campaign 2, i.e., $292 \mathrm{~g}$ versus $245 \mathrm{~g}$, respectively. This was expected because of the higher mixing jet pump velocity for Campaign 2, i.e., $23.9 \mathrm{ft} / \mathrm{s}$ versus $22.9 \mathrm{ft} / \mathrm{s}$, respectively. Also, of interest is the MDT stainless steel distribution at the end of campaign 2 was similar to the difference in accumulated mound solids. That is, after Cycle 10 of Campaign 2 Table 4-5 shows that the North mound was 35.4/9.2 = 3.8 times larger than the South mound, which was similar to the masses of stainless steel collected, i.e., for Table 4-8, 182.9/44.1 $=4.1$ times. Finally, it is 
clear from the data that most of the heaviest particle are concentrated in the two mounds because Campaign 2 showed only $7 \%$ of the material lied outside the mound areas.

\subsection{Stainless Steel Mass Balance}

As was stated in Table 2-1, a full MDT volume was 392 liters (103.6 gal) and in Table 2-3 the solids loading was $100 \mathrm{~g} / 1$, or $39.2 \mathrm{~kg}$ of simulant; and of those solids the stainless steel (SS) had a concentration of $1 \mathrm{wt} \%$, or 392 grams. However, only the first cycle of each campaign, Cycle 1, was the MDT filled with 392 liters. For the remaining nine cycles of each campaign a heel of 68 liters (17.9 gal) was left in the MDT; therefore, it was only refilled with 392 liters -68 liters = 324 liters of simulant containing 324 grams of SS solids. This means that each campaign processed 392 grams $+(9 \times 324)$ grams $=3,308$ grams of stainless steel which left the MDT by three avenues:

1. Batch transfers: Table 4-3 and Table 4-4

2. Core sampling of the accumulated solids mounds: Table 4-6 and Table 4-7

3. Accumulated solids in MDT mounds at the end of a campaign: Table 4-8

The stainless steel used, captured, separated, and measured during and after each campaign are listed in Table 4-9 and Table 4-10

Table 4-9. Stainless steel used in Campaign1

\begin{tabular}{|c|c|c|}
\hline \multicolumn{3}{|c|}{ Campaign 1: Stainless Steel (SS) Mass Balance } \\
\hline Date & Cycle & SS Used \\
grams
\end{tabular}


Table 4-10. Stainless steel used in Campaign 2

\begin{tabular}{|c|c|c|}
\hline \multicolumn{3}{|c|}{ Campaign 2: Stainless Steel (SS) Mass Balance } \\
\hline & & SS Used \\
\hline Date & Cycle & grams \\
\hline $6 / 20 / 2012$ & 1 & 392.0 \\
\hline $6 / 22 / 2012$ & 2 & 324.0 \\
\hline $6 / 25 / 2012$ & 3 & 324.0 \\
\hline $6 / 26 / 2012$ & 4 & 324.0 \\
\hline $6 / 27 / 2012$ & 5 & 324.0 \\
\hline $7 / 2 / 2012$ & 6 & 324.0 \\
\hline $7 / 3 / 2012$ & 7 & 324.0 \\
\hline $7 / 5 / 2012$ & 8 & 324.0 \\
\hline $7 / 6 / 2012$ & 9 & 324.0 \\
\hline $7 / 9 / 2012$ & 10 & 324.0 \\
\hline \multirow[t]{2}{*}{ Total SS Used } & & 3308.0 \\
\hline & & SS Recovered \\
\hline Transferred & & 3469.0 \\
\hline In Core Samples & & 6.8 \\
\hline In MDT after Cycle 10 & & 244.5 \\
\hline Total SS recovered & & 3720.4 \\
\hline \% SS Recovered vs. Used & & $112 \%$ \\
\hline
\end{tabular}

Table 4-9 shows that approximately 3,580 grams of SS were recovered, which is $8.2 \%$ over the added 3308 grams of SS. This recovered SS is a combination of 3,282 grams of transferred SS, 7 grams taken during core sampling, and 292 grams collected from the bottom of the MDT at the end of Campaign 1. Note that a campaign of transferred SS was comprised of filling and emptying the MDT 10 times, each called a cycle. Furthermore, emptying the MDT during each cycle was comprised of 7 transfers to seven receipt tanks. That is, during each campaign there were a total of 70 transfers. For Campaign 1, 4 of the 70 transfers did not separate the SS particles because the magnetic separator was accidentally not engaged, which means the SS estimate would have been higher than the $8 \%$, probably closer to $10 \%$. While the $10 \%$ is well within the acceptance tolerance of $20 \%$ the mass balance was expected to be better.

Similar to Campaign 1, the results from Campaign 2 showed the recovered stainless steel to be more than what was used. Table 4-10 shows that approximately 3,720 grams of SS were recovered, which is $12.5 \%$ over the added 3308 grams of SS. This recovered SS is a combination of 3,469 grams of transferred SS, 7 grams taken during core sampling, and 245 grams collected from the bottom of the MDT at the end of Campaign 2. 
The reason for the larger volume of recovered SS is not from calibrated measurement uncertainty because it was much smaller than $10 \%$, see Table 2-4. It may be that the SS measured and collected had more residual material than expected. All SS in samples were separated using a magnet, washed several times with deionized water; and then separated a second time with a magnet; however, there was some visual evidence that some foreign material remained, e.g. gibbsite, zirconium, sand or precipitated salts.

In an attempt to determine the recovery efficiency of SS solids from samples containing a mixture of simulant solids, a spike sample was made for separation. When collecting samples with a magnet, either by the in-line Magnetic Separator, or by hand, it always contains other solids that need removing. In these samples most of the solids are very definitely SS; however, the exact amount is not known and the concentration most likely changes slightly for each sample. From the experience of processing 70+ samples a mixture was chosen for the spiked sample: $50 \mathrm{~g} \mathrm{SS}$, $12 \mathrm{~g}$ Gibbsite, $2.5 \mathrm{~g} \mathrm{ZrO}_{2}$, and $2.2 \mathrm{~g}$ of sand suspended in the simulant supernatant. The sample was thoroughly mixed and then the SS solids were separated using the same method all previous samples were separated. That is, a hand magnet was first used to separate the SS from the sample, then the separated material was washed several times with deionized water until the water appeared clear, and finally, the magnet was used a second time to separate the washed SS solids from any remaining solids. The wet SS solids were then placed on a pre-weighed paper filter to drain as much liquid as possible and then both the filter and SS were thoroughly dried and weighed. The resulting SS recovered was 51.3 grams, which was $2.6 \%$ heavier than that starting SS mass. While the final solids mass recovered from the spiked sample was not larger than that starting mass by the 8 to $12 \%$ obtained during the campaigns, the spiked sample test did show that despite the attempt to recover only SS solids some other solids persist. Unfortunately, simply mixing four solids in the supernatant is probably not the same as mixing the full simulant in the MDT, transporting to a receipt tank, and collecting a sample. For example, in developing this task settling tests were performed to see how long it would take for the solids to settle to the point the supernatant would be clear enough for the laser measurements to be made through the liquid. The results are shown in Figure 4-71 and depending whether or not the simulant had gibbsite the solids settled at a different rate.

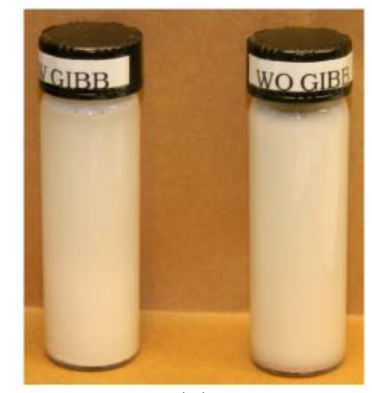

(a)

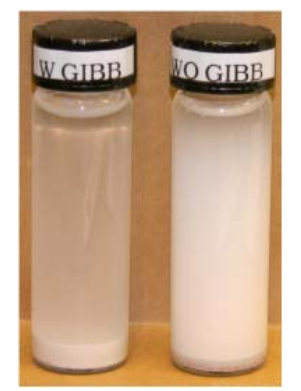

(b)

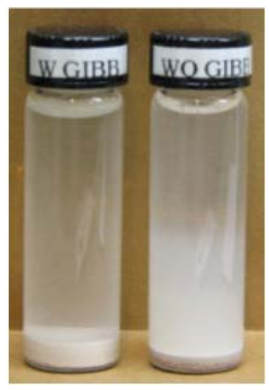

(c)

Figure 4-71. Settling test of SASS simulant with the left vial containing all four solids and the right vial without gibbsite after: (a) 0 hours, (b) 4 hours, and (c) 33.4 hours.

The full simulant settled to an acceptably clear supernatant after approximately 4 hours, but when the gibbsite was left out the simulant was still relatively opaque after 33.5 hours. The may indicated that there was an interaction between the gibbsite and zirconia particle, like flocculation, which caused the settling rate to decrease. This complex interaction may have also translated to 
the entire solids matrix making it harder to separate when the right combination was joined, which may not have existed in the spiked sample. In any case, it is possible that the attraction among the solids may have prevented a good separation even after several campaigns of separation with a magnet and washing leading to higher masses of stainless steel samples that contained other persistent solids.

\subsection{Difference in Mound Sizes}

As seen from all the data, the accumulated solids built up in the MDT at the location furthest from the two jet pump mixers into two mounds with one larger than the other. The reason for the different size mounds was believed to be due to the differences in the strengths of the jet pump. As previous seen, in both campaigns the average velocity of MJP2 was higher than MJP1. For Campaign 1 the difference was approximately 3\% and for Campaign 2 it was approximately 5\%. For both campaigns the rotation of the pumps was synchronized and in the clockwise direction. Each pump head had two jets opposite each other the two pumps were synchronized such that at $0^{\circ}$ the inner jets either faced each other and the outer jets facing $180^{\circ}$ away from each other and towards the tank wall. At $90^{\circ}$ the jets from both pumps formed two lines that were parallel and separated at a distance equal the centerline distance between the two jet pump mixers. The pump rotated in a clockwise direction; therefore, the settling solids were swept in that direction. Figure 4-72 show a sketch of the mounds and jet movements.

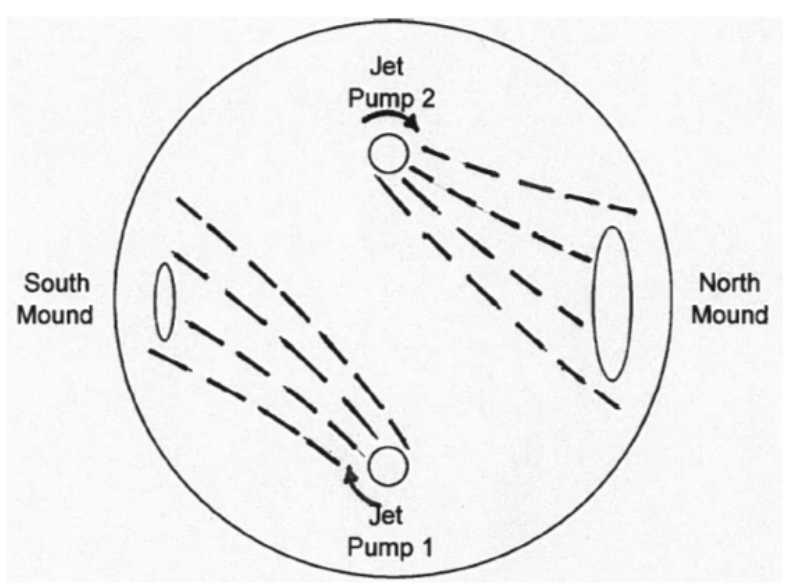

Figure 4-72. MDT showing clockwise rotation of jet pump mixings and influence on mounds.

The jets from both pump mixers move settled solids along the tank bottom and concentration in the location furthest from the sweeping jets. In the clockwise direction the leading edge of North mound is closest to MJP2 and for the South mound it is closed to MJP1. Both mixers deposit and deplete both mounds with solids. However, MJP2 is the principal pump that deposits solids on the leading edge of the North mound and deplete solids from the trailing edge of the South mound,while MJP1 is the principal pump that deposits solids on the leading edge of the South mound and deplete solids from the trailing edge of the North mound. Because the stronger mixer was MJP2 then more solids were deposited on the North than the South mounds and more solids were depleted from the South than the North mounds. 
To test this assumption a short test was performed upon completion of Campaign 2. The MDT was loaded with 3 inches of water and $2000 \mathrm{ml}\left(122 \mathrm{in}^{3}\right)$ of sand and was mixed for 8 minutes, which is when most of the solids movements stabilized. The jet pumps were rotated counterclockwise and synchronized. Over that mixing period the MJP1 velocity averaged at 24.4 $\mathrm{ft} / \mathrm{s}$ and MJP2 velocity average was $27.4 \mathrm{ft} / \mathrm{s}$, that is, JPM2 turn out to be $11 \%$ stronger than MJP1. The tank was then drained of liquid so the mound volumes could be obtained with the laser system and the results are shown in Figure 4-73, Figure 4-74, Figure 4-75, and Figure 4-76. The North mound measured to be $33 \mathrm{in}^{3}$ and the South mound was $56 \mathrm{in}^{3}$, clearly showing the direction of rotation and strength of the mixing pump jets work together to build the mounds.

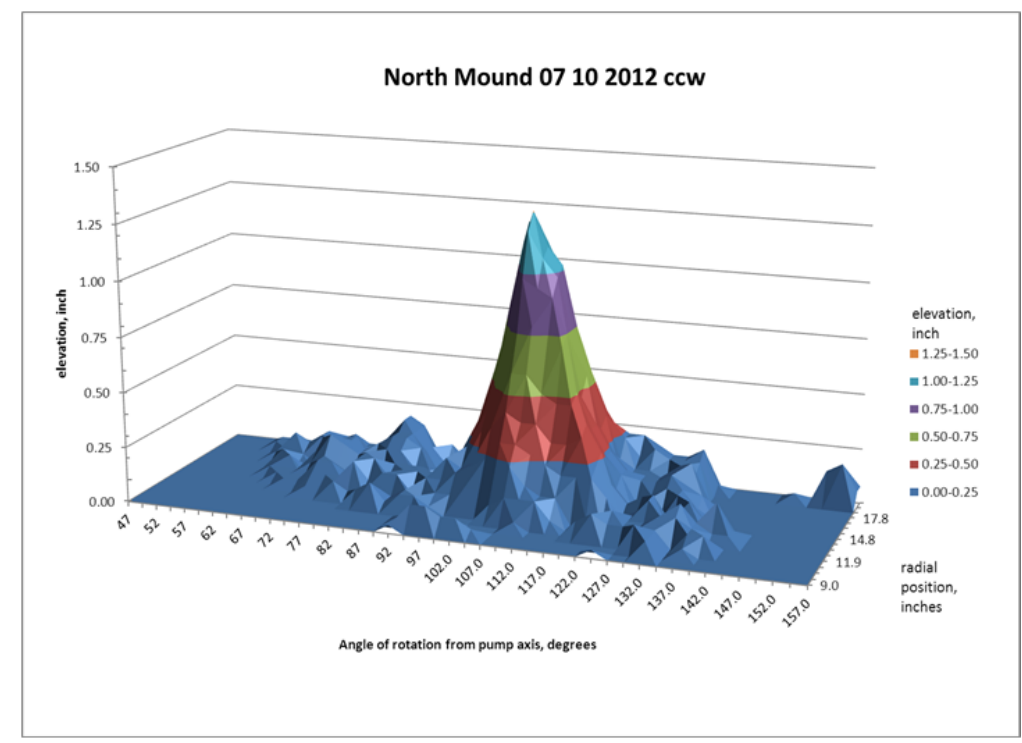

Figure 4-73. North mound side view with counter clockwise rotation of jet pumps Volume of $33 \mathrm{in}^{3}$.

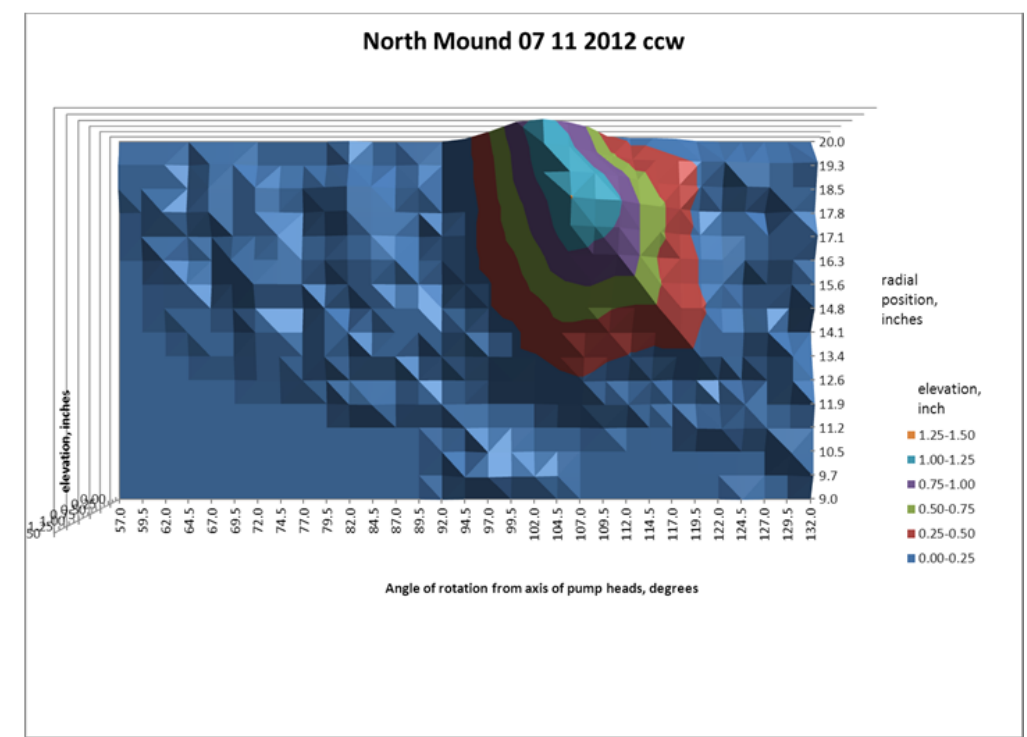

Figure 4-74. North mound top view with counter clockwise rotation of jet pumps Volume of $33 \mathrm{in}^{3}$. 
Revision 0

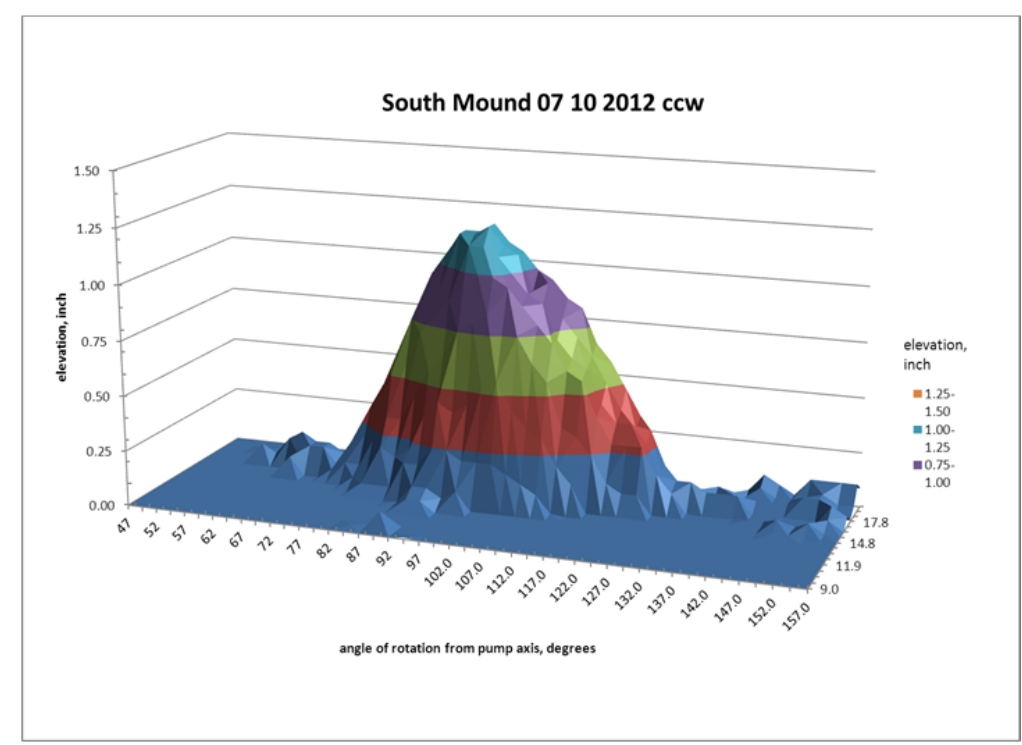

Figure 4-75. South mound side view with counter clockwise rotation of jet pumps Volume of $56 \mathrm{in}^{3}$.

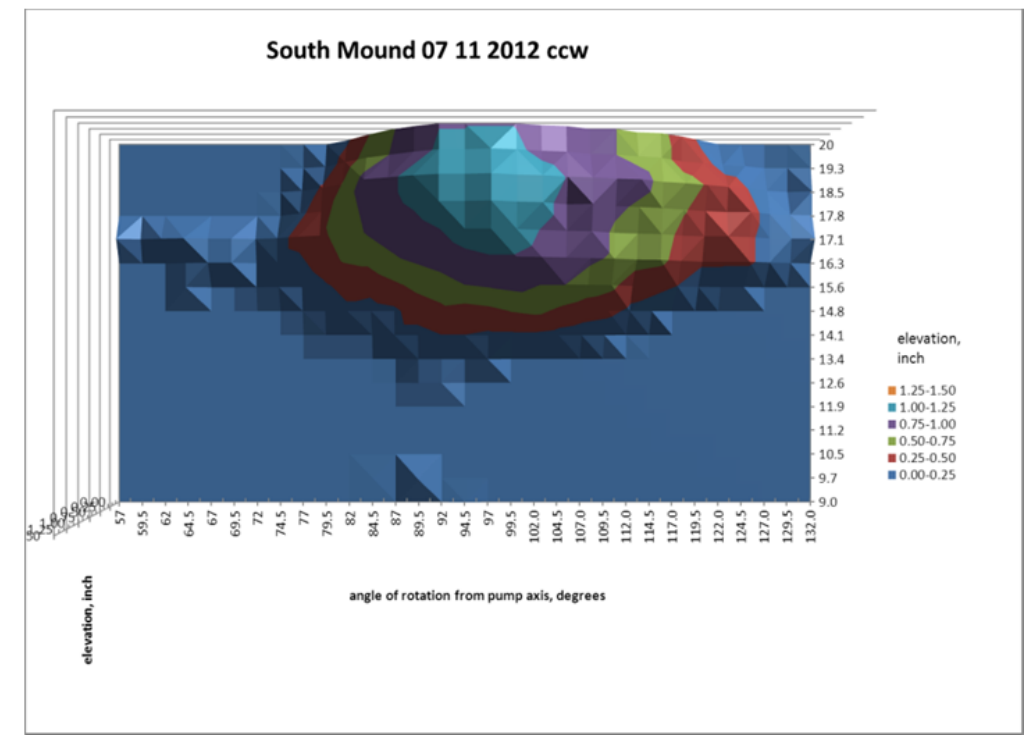

Figure 4-76. South mound top view with counter clockwise rotation of jet pumps Volume of 56 in $^{3}$. 


\subsection{Conclusions}

Several techniques were developed and equipment designed to accomplish the measurements needed, which include:

1. Magnetic particle separator to remove simulant stainless steel solids. A device was designed and built to capture these solids, which represent heavier solids during a waste transfer from a staging tank. [Figure 3-3]

2. Photographic equipment to determine the volume of the solids mounds. The mounds were photographed as they were exposed at different tank waste levels to develop a composite of topographical areas. [Figure 3-12]

3. Laser rangefinders to determine the volume of the solids mounds. The mounds were scanned after tank supernatant was removed. [Figure 3-8]

4. Core sampler to determine the stainless steel solids distribution within the solids mounds. This sampler was designed and built to remove small sections of the mounds to evaluate concentrations of the stainless steel solids at different special locations. [Figure 3-15]

5. Computer driven positioner that placed the laser rangefinders and the core sampler in appropriate locations over solids mounds that accumulated on the bottom of a scaled staging tank where mixing is poor. [Figure 3-6Figure 3-7]

These devices and techniques were effective to estimate the movement, location, and concentrations of the solids representing heavier particles, e.g., plutonium, and could perform well at a larger scale

Over the 26 days of testing the following observations were made:

1. Fast settling particles, i. e, $\sim 300 \mu \mathrm{m}$ sand, transported out of the test tank readily and only accumulated during the first two to three cycles. All others cycles transported about the same amount of sand. [Figure 4-6 and Figure 4-7]

2. Very fast settling particles, i.e., $\sim 125 \mu \mathrm{m}$ stainless steel, transported readily but did accumulate until about the $3^{\text {rd }}$ to $4^{\text {th }}$ cycle, after which most of the additional solids introduced into the tanks were transported out. [Figure 4-10 and Figure 4-13]

3. Solids did accumulate in the two "dead zones" of the tank, i.e., the locations opposite of the jet pump mixers and accumulations occurred almost immediate on mixing the tank, i.e., before transfer began. [Example: Figure 4-20 and Figure 4-20]

4. The solid mounds that formed, referred to as the North and South mounds, were of unequal size with the North mound approximately three times the volume of the South mound. This volume difference was due to the different strengths for the two mixing jet pump velocities. That is, Jet Mixing Pump 2 was approximately $1 \mathrm{ft} / \mathrm{s}$ stronger that Jet Mixing Pump 1 [Figure 2-7] (or approximately 0.35 gpm stronger for these scaled pumps). This fact was demonstrated when a subsequent test was performed by rotating the pumps in the opposite direction, counterclockwise, resulting in the South mound become larger than the North mound. [Figure 4-74 and Figure 4-76]

5. Accumulated volumes determined by the two methods employed, i.e., laser and photographic, agreed on the average to within $22 \%$ for the North mound and $83 \%$ for the 
South mound. This measurement difference was not unexpected because the mounds were small, less than 1 inch in height for the North mounds and less than 0.5 inch in height for the South mounds. Furthermore, the small sized solids, i.e., gibbsite and zirconia, covered everything making the contrast between the supernatant and solids difficult for the photographic technique to clearly distinguish the liquid-solids interfaces. The smaller the volume and the poorer surface contrast the more measurement uncertainty for the photographic technique. [Table 4-5]

6. Core samples taken from the mounds clearly indicated that stainless steel is the predominant component of the bottom of the settled volumes. The composition gradually transitions to sand in the upper surface of the mounds. [Table 4-6 and Table 4-7]

7. At the end of 10 cycles for each of the two campaigns the amount of stainless steel that remained in the staging tank was 9.0\% and 7.6\%, respectively.[Table 4-9 and Table 4-10]

8. In Campaign 2 , the $93 \%$ residual stainless steel was shown to be located within the mounds of accumulated solids with the remaining $7 \%$ in all other locations. [Table 4-8]

\subsection{Recommendations}

Based on the experience from the experiment the following recommendations can be made"

1. The magnetic separator should be used in future testing because it did an excellent job of separating the stainless steel particles from the simulant transported out of the MDT. Its separation efficiency appeared to be $100 \%$, and had minimal impact on the overall operation of the experiment. However, the mass balance after each campaign for stainless steel (SS) indicated that the SS recovered was approximately $10 \%$ more than what was in the simulant. While the SS solids recovered were washed and separated several times to remove interstitial solids and salts it appears that some non-stainless steel material was included in the mass balances. A more throughout clean protocol will be necessary if this separation technique is employed to measure the mass of stainless steel.

2. The core sampler should be used in future testing because it did an excellent job of obtaining samples of the solids in the accumulated mounds as long as solids stayed damp, but not very wet. The dampness kept the solid plugs intact, but too much liquid allows the plugs either not to be captured properly or to break up before they can be properly captured.

3. For future testing the most accurate measurements of mound shapes and volumes can be obtained with the laser technique. Once set up the laser technique requires very little labor. The photographic technique will become more accurate as the mounds become larger and the liquid-solids contrast increases. The photographic technique requires much more labor and analysis. An uncertainty analysis should be done to quantify the uncertainty before deciding on the method to use.

4. The solids, especially those with the smallest particle sizes, i.e., gibbsite and zirconia, present many pluggage problems in tubes, pipes, and any flat sections when the simulant velocity drops or stops for more than 5 to 10 minutes. Consideration should be given to those locations on how recovery from a plug will be accomplished or avoided to minimize operational disruptions. See Appendix C, Lessons Learned, "Pluggage Problems," for a further discussion on this issue. 
5. The slurry pump system that fed the two Jet Pump Mixers in the Mixing Demonstration Tank should be redesigned to eliminate the observed flow fluctuations for any future tests at SRNL.

\subsection{References}

1. Inter-Entity Work Order, 2012, "Solids Accumulation Scouting Studies," Work Order No. M0SRV00070, January 31.

2. Duignan, M.R., Steeper, T.J., Steimke, J.L., Leishear, R.A., 2012. "Solids Accumulation Scouting Studies," Task Technical and Quality Assurance Plan, Doc. No. SRNL-RP-2012-00057.

3. Lee, K.P., 2012. "Waste Feed Delivery Mixing and Sampling Program Limits of Performance and Solids Accumulation Scouting Studies Test Plan," Doc. No. RPPPLAN-52005, Rev. 0

4. Thien, M.G., Sexton, R.A., 2012. "Waste Feed Delivery Mixing and Sampling Program Plan and Test Requirements," RPP-PLAN-41807, Rev. 1.

5. Adamson, D.J., Poirier, M.R., Steeper, T.J. 2009. "Demonstration of Simulated Waste Transfers from Tank AY-102 to the Hanford Waste Treatment Facility," Doc. No. SRNL-STI-2009-00717, Rev. 0.

6. Adamson, D.J., Restivo, M.L., Steeper, T.J., Greer, D.A. 2010. "Demonstration of Mixer Jet Pump Rotational Sensitivity on Mixing and Transfers of the AY-102 Tank," Doc. No. SRNL-STI-2010-00521, Rev. 0.

7. Adamson, D.J., Gauglitz, P.A. 2011. "Demonstration of Mixer and Transferring Settling Cohesive Slurry Simulants in the AY-102 Tank," Doc. No. SRNL-STI-201100278, Rev. 0.

8. Lee, K.P., Wells, B.E., Gauglitz, P.A., and Sexton, R.A., 2012. "Waste Feed Delivery Mixing and Sampling Program Simulant Definition for Tank Farm Performance Testing," RPP-PLAN-51625, Rev.0.

9. Duignan, M.R., Steeper, T.J., Steimke, J.L., Fowley, M.D., 2012. "Simulant Development and Sampling Plan.” Memorandum No. SRNL-L3100-2012-00024, Rev. 0 .

10. 2011. "Interface Control Document for Waste Feed," 24590-WTP-ICD-MG01-019, Rev. 5 Bechtel National, Inc. Richland, Washington.

11. Herting, D.L. to Nelson, E.A., 2007. "Preparation of Simulated SST Early Feed Solution for Pilot Plant," Doc. No. CH2M-0701541.

12. Duignan, M.R., Steeper, T.J., Steimke, J.L., Fowley, M.D., 2012. "Test Plan - Solids Accumulation Scouting Studies," Doc. No SRNL-STI-2012-00239, Rev. 0

13. Kaplan, W., 1952. Advanced Calculus, Addison-Wesley Pub. Co."

14. Duignan, M. R. 2012, "Solids Accumulation Scouting Studies," SRNL Notebook No. SRNL_NB-00033. 


\title{
Appendix A: Particle Size Distribution for Simulant Solids
}

\author{
Gibbsite \\ Zirconium Oxide \\ Sand \\ $\&$
}

Stainless Steel (with and without sonication) 


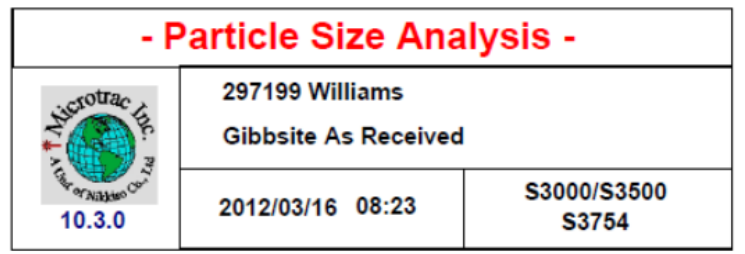

\begin{tabular}{|c|c|}
\hline Data Item & Value \\
\hline MV(um): & 11.4 \\
\hline MN(um): & 0.778 \\
\hline MA(um): & 4.31 \\
\hline CS: & 1.391 \\
\hline SD: & 8.16 \\
\hline & \\
\hline
\end{tabular}

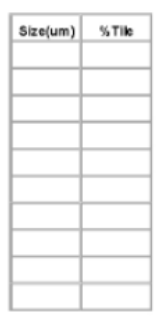

\begin{tabular}{|c|c|}
\hline \%Tile & Size(um) \\
\hline 10.00 & 1.750 \\
\hline 20.00 & 3.51 \\
\hline 25.00 & 4.41 \\
\hline 40.00 & 7.44 \\
\hline 50.00 & 9.34 \\
\hline 60.00 & 12.22 \\
\hline 70.00 & 14.68 \\
\hline 75.00 & 16.04 \\
\hline 90.00 & 22.34 \\
\hline 95.00 & 27.68 \\
\hline
\end{tabular}

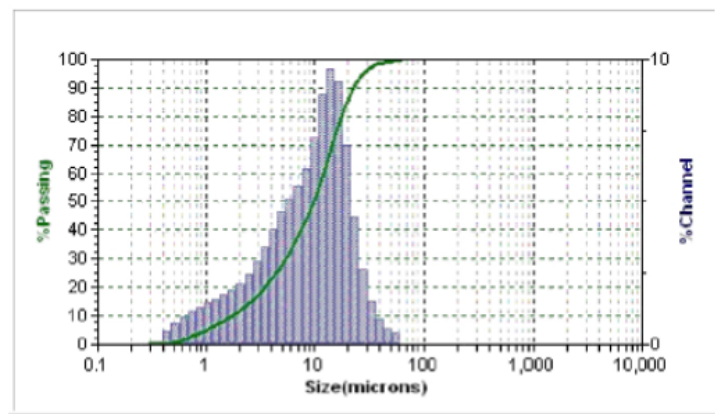

in $\mathrm{DiH} 2 \mathrm{O}$
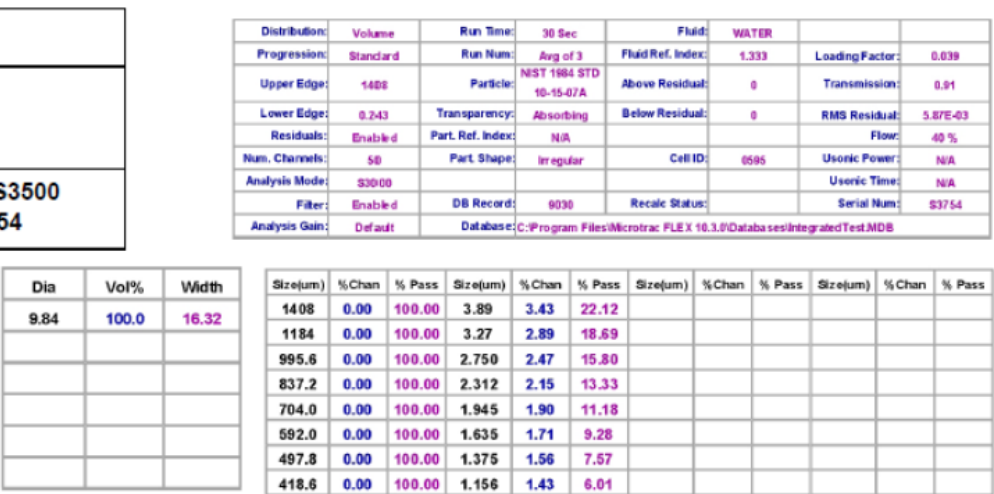

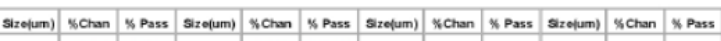
\begin{tabular}{|l|l|l|l|l|l|l|l|}
\hline 1408 & 0.00 & 100.00 & 3.89 & 3.43 & 22.12 \\
\hline & 0.09 & 100.00 & 3.27 & 2.89 & 18.59 \\
\hline
\end{tabular}

\begin{tabular}{|l|l|l|l|l|l|}
\hline 1184 & 0.00 & 100.00 & 3.27 & 2.89 & 18.59 \\
\hline
\end{tabular}

\begin{tabular}{|l|l|l|l|l|l|l|}
\hline $\mathbf{9 9 5 . 6}$ & $\mathbf{0 . 0 0}$ & 100.00 & $\mathbf{2 . 7 5 0}$ & 2.47 & 15.80 \\
\hline
\end{tabular}

\begin{tabular}{|l|l|l|l|l|l|l|}
\hline 837.2 & 0.00 & 100.00 & $\mathbf{2 . 3 1 2}$ & $\mathbf{2 . 1 5}$ & 13.33 \\
\hline 704 & 0.00 & 100.00 & 1.965 & 1.90 & 11.13 \\
\hline
\end{tabular}

\begin{tabular}{|l|l|l|l|l|l|l|}
\hline 704.0 & 0.00 & 100.00 & 1.945 & 1.90 & 11.18 \\
\hline
\end{tabular}

\begin{tabular}{|l|l|l|l|l|l|}
\hline 592.0 & 0.00 & 100.00 & 1.635 & 1.71 & 9.28 \\
\hline & 0.00 & 100.00 & 1.375 & 1.56 & 1.58 \\
\hline
\end{tabular}

\begin{tabular}{|l|l|l|l|l|l|}
\hline 497.8 & 0.00 & 100.00 & 1.375 & 1.56 & 7.57 \\
\hline
\end{tabular}

\begin{tabular}{|l|l|l|l|l|l|l|}
\hline 418.6 & 0.00 & 100.00 & 1.156 & 1.43 & 6.01 \\
\hline
\end{tabular}

\begin{tabular}{|l|l|l|l|l|l|l|}
\hline 352.0 & 0.00 & 100.00 & 0.972 & 1.30 & 4.58 \\
\hline
\end{tabular}

\begin{tabular}{|l|l|l|l|l|l|}
\hline 296.0 & 0.00 & 100.00 & 0.818 & 1.15 & 3.28 \\
\hline
\end{tabular}

\begin{tabular}{|l|l|l|l|l|l|}
\hline 248.9 & 0.00 & 100.00 & 0.688 & 0.96 & 2.13 \\
\hline
\end{tabular}

\begin{tabular}{|l|l|l|l|l|l|}
\hline 209.3 & 0.00 & 100.00 & 0.578 & 0.71 & 1.17 \\
\hline
\end{tabular}

\begin{tabular}{|l|l|l|l|l|l|l|}
\hline $\mathbf{1 7 6 . 0}$ & $\mathbf{0 . 0 0}$ & 100.00 & 0.486 & 0.46 & 0.46 \\
\hline 15.0 & 0.00 & 100.00 & 0.409 & 0.00 & 0.00 \\
\hline
\end{tabular}

\begin{tabular}{|l|l|l|l|l|l|l|}
\hline 148.0 & 0.00 & 100.00 & 0.409 & 0.00 & 0.00 \\
\hline
\end{tabular}

\begin{tabular}{|l|l|l|l|l|l|l|}
\hline 124.5 & 0.00 & 100.00 & 0.344 & 0.00 & 0.00 \\
\hline
\end{tabular}

\begin{tabular}{|l|l|l|l|l|l|l|}
\hline 104.7 & 0.00 & 100.00 & 0.2890 & 0.00 & 0.00 \\
\hline
\end{tabular}

\begin{tabular}{|l|l|l|l|}
\hline 88.00 & 0.00 & 100.00 \\
\hline 74.00 & 0.00 & 1000
\end{tabular}

\begin{tabular}{|l|c|c|}
\hline $\mathbf{7 4 . 0 0}$ & $\mathbf{0 . 0 0}$ & 100.00 \\
\hline 62.00 & 0.09 & 100.00
\end{tabular}

\begin{tabular}{|l|l|l|}
\hline 62.23 & 0.39 & 100.00 \\
\hline 6233 & 0.59 & 99.61 \\
\hline
\end{tabular}

\begin{tabular}{|l|l|l|}
\hline 52.33 & 0.58 & 99.61 \\
\hline
\end{tabular}

\begin{tabular}{|l|l|l|}
\hline 44.00 & $\mathbf{0 . 9 1}$ & 99.03 \\
\hline
\end{tabular}

\begin{tabular}{|l|l|l|}
\hline 37.00 & 1.51 & 98.12 \\
\hline 31.11 & 2.51 & 96.61 \\
\hline
\end{tabular}

\begin{tabular}{|l|l|l|}
\hline 31.11 & 2.61 & 96.61 \\
\hline
\end{tabular}

\begin{tabular}{|l|l|l|l|}
\hline 26.16 & 4.47 & 94.00 \\
\hline & 2.00 & 7.00 & 9.55
\end{tabular}

\begin{tabular}{|l|l|l|}
\hline 22.00 & 7.00 & 89.53 \\
\hline
\end{tabular}

\begin{tabular}{|l|l|l|}
\hline 18.50 & 9.22 & 82.53 \\
\hline 15.56 & 9.69 & 73.31 \\
\hline
\end{tabular}

\begin{tabular}{|l|l|l|}
\hline 15.56 & 9.69 & 73.31
\end{tabular}

\begin{tabular}{|l|l|l|}
\hline 13.08 & 8.77 & 63.62 \\
\hline 11.00 & 7.26 & 54.85
\end{tabular}

\begin{tabular}{|l|l|l|}
\hline 11.00 & 7.26 & 54.85 \\
\hline 9.25 & 6.14 & 47.59
\end{tabular}

\begin{tabular}{|l|l|l|l|}
\hline 9.25 & 6.14 & 47.59 \\
\hline 7.78 & 5.52 & 4.95 \\
\hline
\end{tabular}

\begin{tabular}{|l|l|l|l|}
\hline 7.78 & 5.52 & 41.45 \\
\hline 6.59 & 5.11 & 35.93
\end{tabular}

\begin{tabular}{|l|l|l|}
\hline 6.54 & 5.11 & 35.93 \\
\hline 550 & 4.50 & 30.92
\end{tabular}

\begin{tabular}{|l|l|l|}
\hline 5.50 & 4.65 & 30.32 \\
\hline 4.62 & 4.05 & 25.17 \\
\hline
\end{tabular}

Warnings: NONE

Figure A-0-1. PSD for gibbsite in $\mathrm{H} 2 \mathrm{O}$ and with no sonication 
Revision 0

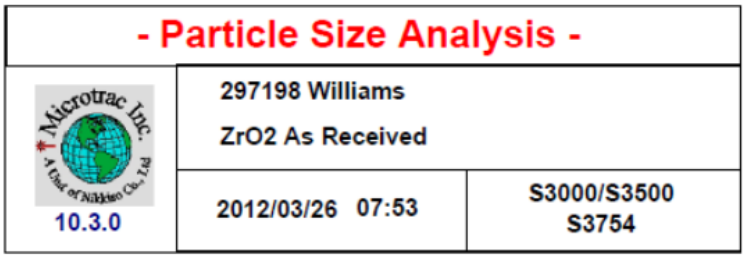

\begin{tabular}{|c|c|}
\hline Data Item & Value \\
\hline MV(um): & 15.41 \\
\hline MN(um): & 0.442 \\
\hline MA(um): & 2.486 \\
\hline CS: & 2.414 \\
\hline SD: & 13.84 \\
\hline & \\
\hline
\end{tabular}

\begin{tabular}{|c|c|}
\hline \%Tile & Size(um) \\
\hline 10.00 & 0.733 \\
\hline 20.00 & 1.772 \\
\hline 25.00 & 2.532 \\
\hline 40.00 & 6.80 \\
\hline 50.00 & 12.02 \\
\hline 60.00 & 17.01 \\
\hline 70.00 & 21.38 \\
\hline 75.00 & 23.73 \\
\hline 90.00 & 34.30 \\
\hline 95.00 & 42.38 \\
\hline
\end{tabular}

\begin{tabular}{|c|c|c|}
\hline Dia & Vol\% & Width \\
\hline 13.95 & 92.9 & 27.72 \\
\hline 0.433 & 7.1 & 0.20 \\
\hline & & \\
\hline & & \\
\hline & & \\
\hline & & \\
\hline
\end{tabular}

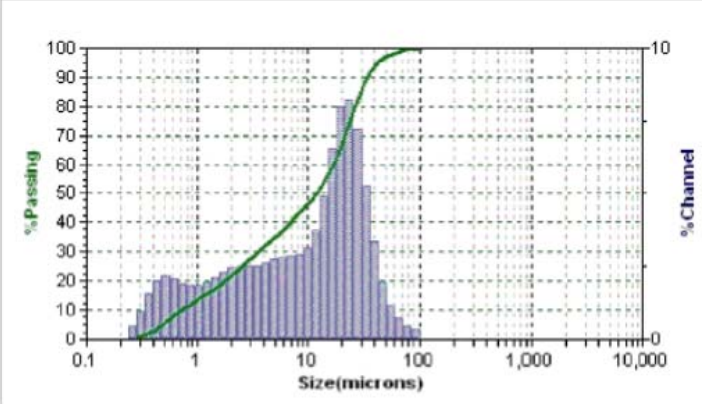

in $\mathrm{DiH} 2 \mathrm{O}$

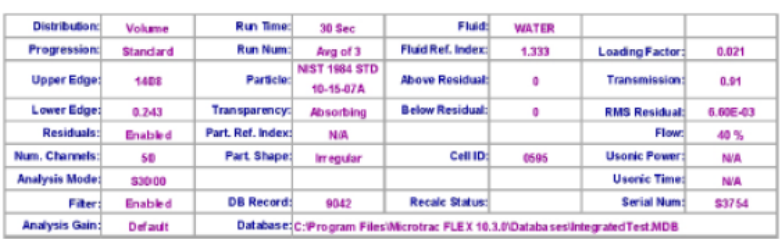

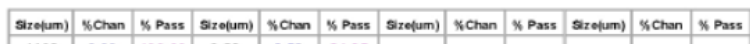
\begin{tabular}{|c|c|c|c|c|c|}
\hline 1408 & 0.00 & 100.00 & 3.89 & 2.53 & 31.25 \\
\hline 1184 & 0.00 & 100.00 & 3.27 & 2.52 & 28.72 \\
\hline
\end{tabular}

\begin{tabular}{|c|c|c|c|c|c|}
\hline 1184 & 0.00 & 100.00 & 3.27 & 2.52 & 28.72 \\
\hline 95.6 & 0.00 & 100.00 & 2.75 & 2.51 & 26.20 \\
\hline
\end{tabular}

\begin{tabular}{|l|l|l|l|l|l|l|}
\hline 995.6 & 0.00 & 100.00 & 2.750 & 2.51 & 26.20 \\
\hline
\end{tabular} \begin{tabular}{|l|l|l|l|l|l|}
\hline 837.2 & 0.00 & 100.00 & 2.312 & 2.44 & 23.50 \\
\hline 8040 & 0.00 & 100.00 & 1.945 & 2.29 & 21.25 \\
\hline
\end{tabular}

\begin{tabular}{|l|l|l|l|l|l|}
\hline 837.2 & 0.00 & 100.00 & 2.312 & 2.44 & 23.69 \\
\hline 704.0 & 0.00 & 100.00 & 1.945 & 2.29 & 21.25 \\
\hline 592.0 & 0.00 & 100.00 & 1.63 & 2.12 & 10.96 \\
\hline
\end{tabular}

\begin{tabular}{|l|l|l|l|l|l|}
\hline $\mathbf{7 0 4 . 0}$ & $\mathbf{0 . 0 0}$ & 100.00 & $\mathbf{1 . 9 4 5}$ & $\mathbf{2 . 2 9}$ & 21.25 \\
\hline $\mathbf{5 9 2 . 0}$ & $\mathbf{0 . 0 0}$ & 100.00 & $\mathbf{1 . 6 3 5}$ & $\mathbf{2 . 1 2}$ & 18.96 \\
\hline $\mathbf{4 9 7 . 0}$ & 0.00 & 100.00 & $\mathbf{1 . 3 5}$ & 1.95 & 10.84 \\
\hline
\end{tabular}

\begin{tabular}{|l|l|l|l|l|l|l|}
\hline $\mathbf{4 9 7 . 8}$ & $\mathbf{0 . 0 0}$ & 100.00 & $\mathbf{1 . 3 7 5}$ & 1.95 & 16.84 \\
\hline
\end{tabular}

\begin{tabular}{|l|l|l|l|l|l|l|}
\hline 418.6 & 0.00 & 100.00 & 1.156 & 1.85 & 14.89 \\
\hline
\end{tabular}

\begin{tabular}{|l|l|l|l|l|l|}
\hline 352.0 & 0.00 & 100.00 & 0.972 & 1.34 & 13.04 \\
\hline
\end{tabular}

\begin{tabular}{|l|l|l|l|l|l|}
\hline 296.0 & 0.00 & 100.00 & 0.818 & 1.92 & 11.20 \\
\hline
\end{tabular}

\begin{tabular}{|l|l|l|l|l|l|}
\hline 248.9 & 0.00 & 100.00 & 0.688 & 2.08 & 9.28 \\
\hline 209.3 & 0.00 & 1000 & 0.578 & 2.18 & 7.20 \\
\hline
\end{tabular}

\begin{tabular}{|l|l|l|l|l|l|l|}
\hline 209.3 & 0.00 & 100.00 & 0.578 & 2.18 & 7.20 \\
\hline 17.0 & 0.00 & 100.00 & 0.486 & 2.09 & 5.02 \\
\hline
\end{tabular}

\begin{tabular}{|l|l|l|l|l|l|l|}
\hline 176.0 & 0.00 & 100.00 & 0.486 & 2.03 & 5.02 \\
\hline
\end{tabular}

\begin{tabular}{|l|l|l|l|l|l|l|}
\hline 148.0 & 0.00 & 100.00 & 0.409 & 1.56 & 2.99 \\
\hline
\end{tabular}

\begin{tabular}{|l|l|l|l|l|l|l|}
\hline 124.5 & 0.00 & 100.00 & 0.344 & 0.96 & 1.43 \\
\hline & 0.31 & 100.00 & 0.2090 & 0.47 & 0.47 \\
\hline
\end{tabular}

\begin{tabular}{|l|l|l|l|l|l|}
\hline 104.7 & 0.31 & 100.00 & 0.2890 & 0.47 & 0.47 \\
\hline
\end{tabular}

\begin{tabular}{|l|l|l|l|}
\hline 88.00 & 0.45 & 99.69 \\
\hline
\end{tabular}

\begin{tabular}{|l|l|l|}
74.00 & 0.70 & 99.24 \\
\hline 62.23 & 1.15 & 90.54
\end{tabular}

\begin{tabular}{|l|l|l|}
\hline 62.23 & 1.15 & 98.54 \\
\hline 52.33 & 1.90 & 97.39
\end{tabular}

\begin{tabular}{|l|l|l|}
\hline 52.33 & 1.98 & 97.39 \\
\hline
\end{tabular}

\begin{tabular}{|l|l|l|}
\hline 44.00 & 3.35 & 95.41 \\
\hline 3700 & 5.25 & 92.06
\end{tabular}

\begin{tabular}{|l|l|l|}
\hline 37.00 & 5.25 & 92.06 \\
\hline
\end{tabular}

\begin{tabular}{|l|l|l|}
\hline 31.11 & 7.23 & 86.81 \\
\hline
\end{tabular}

\begin{tabular}{|l|c|c|c|}
\hline $\mathbf{2 6 . 1 6}$ & $\mathbf{8 . 1 9}$ & 79.58 \\
\hline
\end{tabular}

\begin{tabular}{|l|l|l|}
\hline 22.00 & 8.01 & 71.39 \\
\hline
\end{tabular}

\begin{tabular}{|l|l|l|}
\hline 18.50 & 6.54 & 63.38 \\
\hline
\end{tabular}

\begin{tabular}{|l|l|l|}
\hline 15.56 & 4.90 & 56.84 \\
\hline
\end{tabular}

\begin{tabular}{|l|l|l|}
\hline 13.08 & 3.73 & 51.94 \\
\hline 11.00 & 3.12 & 48.21 \\
\hline
\end{tabular}

\begin{tabular}{|c|c|c|}
\hline 11.00 & 3.12 & 48.21 \\
\hline 9.25 & 2.09 & 45.09 \\
\hline
\end{tabular}

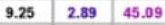

\begin{tabular}{|l|l|l|}
7.78 & 2.84 & 42.20 \\
\hline 654 & 2.25 & 39.36
\end{tabular}

\begin{tabular}{|l|l|l|l|}
\hline 6.54 & 2.80 & 39.36 \\
\hline
\end{tabular}

\begin{tabular}{|l|l|l|}
\hline 5.50 & 2.71 & 36.56 \\
\hline
\end{tabular}

\begin{tabular}{|l|l|l|}
\hline 4.62 & 2.60 & 33.85 \\
\hline
\end{tabular}

Warnings: NONE

Figure A-0-2. PSD for zirconium oxide in $\mathrm{H} 2 \mathrm{O}$ and with no sonication. 
Solids Accumulation Scouting Studies

SRNL-STI-2012-00508

Revision 0

\begin{tabular}{|c|c|c|}
\hline \multicolumn{3}{|c|}{ - Particle Size Analysis - } \\
\hline ? & $\begin{array}{l}298136 \text { Williams } \\
\text { Safety Sand As R }\end{array}$ & \\
\hline $\begin{array}{l}\text { Prines of } \\
10.3 .0\end{array}$ & $2012 / 03 / 29 \quad 09: 43$ & $\begin{array}{c}\mathrm{S} 3000 / \mathrm{S} 3500 \\
\mathrm{~S} 3754\end{array}$ \\
\hline
\end{tabular}

\begin{tabular}{|c|c|c|c|c|c|c|c|}
\hline Distribiouon & Volume & Rin Time: & $30 \mathrm{sec}$ & Fled & WATER & & \\
\hline Progrestion & Standard & Run Num: & Aorg of 3 & FisidRet, index & 1.33 & Loading Factor: & 0.286 \\
\hline Upper Edge: & $10 \mathrm{E}$ & Paracie: & $\begin{array}{c}\text { NSTT 1.84 STD } \\
\text { 10.15.07A }\end{array}$ & Above Residuat: & 0 & Tranumisstion: & 0.97 \\
\hline Lomer Edope: & 0.240 & Transparmery. & Nosorting & Below Residuat: & 0 & RMS Residuat: & 7.11Ee? \\
\hline Residuals: & Enabted & Part Ret. index: & Na & & & Flow & $40 \%$ \\
\hline Mum. charnets: & so & Part stope: & inequar & Celllo: & 0695 & Usorkic Power: & NaA \\
\hline Andlyis Mode: & 53000 & & & & & Userie Time: & Na \\
\hline Fater: & Enabibed & DB Record: & 9074 & Recales Salus: & & Serial thum: & $\sin 54$ \\
\hline Andyes Gain: & Defadt & & & & tatathat & rated Teat MDes & \\
\hline
\end{tabular}

\begin{tabular}{|c|c|}
\hline Data Item & Value \\
\hline MV(um): & 293 \\
\hline MN(um): & 217.9 \\
\hline MA(um): & 268.8 \\
\hline CS: & $2.23 E-02$ \\
\hline SD: & 78.61 \\
\hline & \\
\hline
\end{tabular}

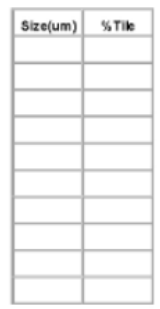

\begin{tabular}{|c|c|}
\hline \%Tile & Size(um) \\
\hline 10.00 & 195.1 \\
\hline 20.00 & 224.1 \\
\hline 25.00 & 235.5 \\
\hline 40.00 & 265.3 \\
\hline 50.00 & 284.2 \\
\hline 60.00 & 304.5 \\
\hline 70.00 & 327.5 \\
\hline 75.00 & 340.7 \\
\hline 90.00 & 401.2 \\
\hline 95.00 & 444.6 \\
\hline
\end{tabular}

\begin{tabular}{|c|c|c|}
\hline Dia & Vol\% & Width \\
\hline 284.2 & 100.0 & 157.20 \\
\hline & & \\
\hline & & \\
\hline & & \\
\hline & & \\
\hline & & \\
\hline
\end{tabular}

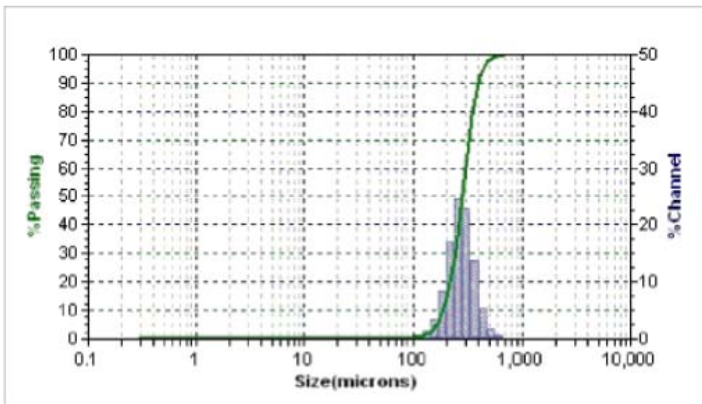

in $\mathrm{DiH} 2 \mathrm{O}$

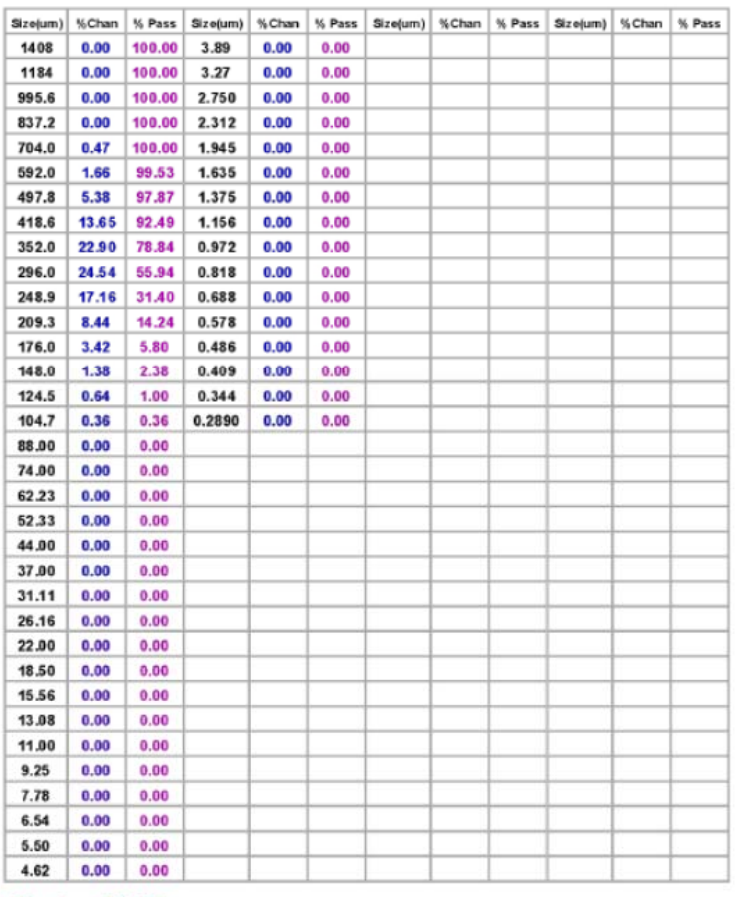

Warnings: NONE

Figure A-0-3. PSD for yellow sand in $\mathbf{H 2 O}$ and with no sonication 
Revision 0
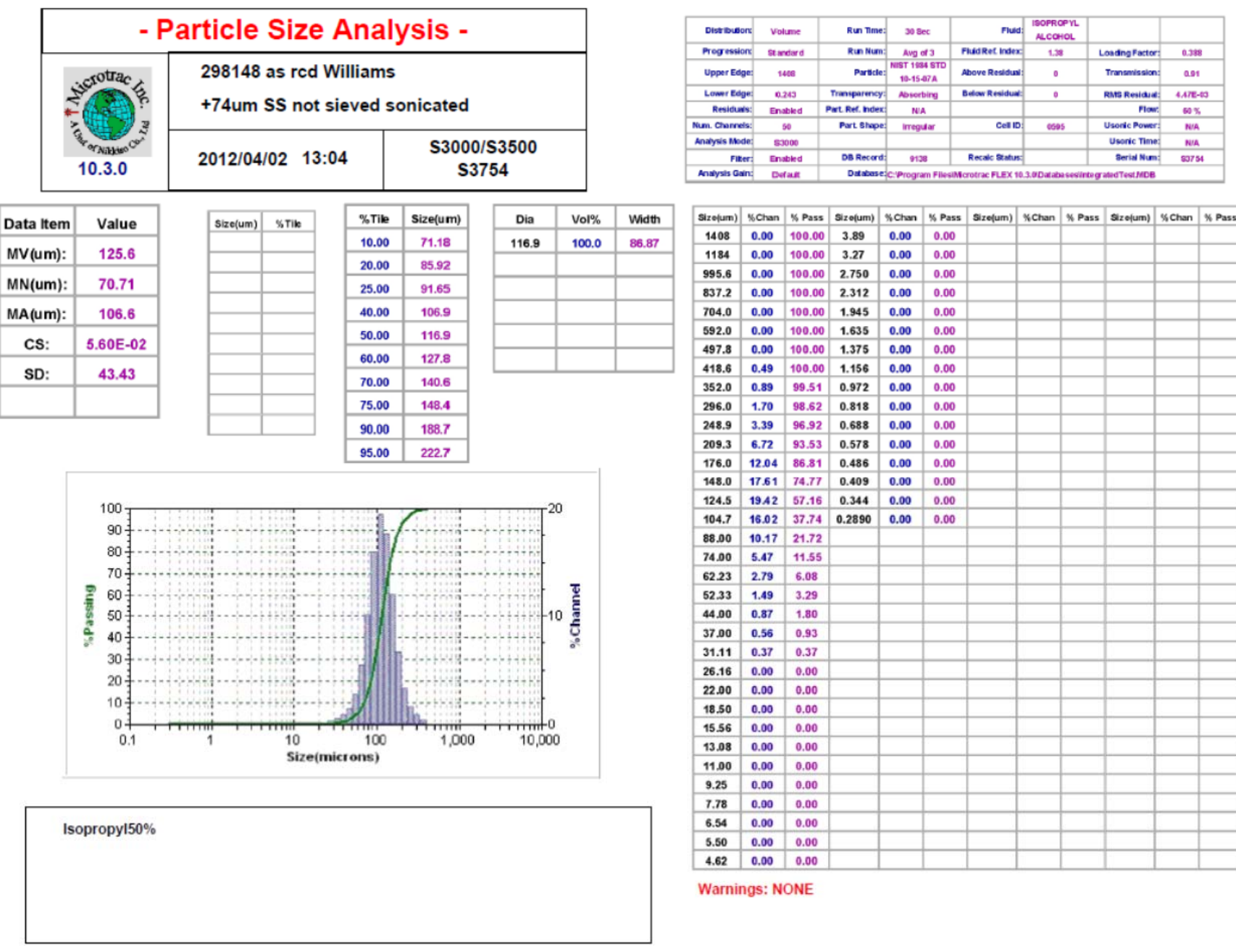

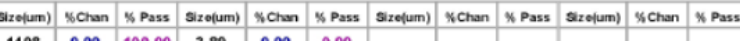
\begin{tabular}{|l|l|l|l|l|l|}
\hline 1408 & 0.00 & 100.00 & 3.89 & 0.00 & 0.00 \\
\hline
\end{tabular}

\begin{tabular}{|l|l|l|l|l|l|l|}
\hline 1184 & 0.00 & 100.00 & 3.27 & 0.00 & 0.00 \\
\hline
\end{tabular}

\begin{tabular}{|l|l|l|l|l|l|}
\hline 995.6 & 0.00 & 100.00 & 2.750 & 0.00 & 0.00 \\
\hline
\end{tabular}

\begin{tabular}{|l|l|l|l|l|l|}
\hline $\mathbf{8 3 7 . 2}$ & $\mathbf{0 . 0 0}$ & 100.00 & $\mathbf{2 . 3 1 2}$ & $\mathbf{0 . 0 0}$ & $\mathbf{0 . 0 0}$ \\
\hline
\end{tabular}

\begin{tabular}{|l|l|l|l|l|l|l|}
\hline 704.0 & 0.00 & 100.00 & 1.945 & 0.00 & 0.00 \\
\hline 592.0 & 0.00 & 100.00 & 1.655 & 0.00 & 0.00 \\
\hline
\end{tabular}

\begin{tabular}{|l|l|l|l|l|l|l|}
\hline 592.0 & 0.00 & 100.00 & 1.635 & 0.00 & 0.00 \\
\hline 497.8 & 0.00 & 100.00 & 1.375 & 0.00 & 0.00 \\
\hline
\end{tabular}

\begin{tabular}{|l|l|l|l|l|l|l|}
\hline 497.8 & 0.00 & 100.00 & 1.375 & 0.00 & 0.00 \\
\hline
\end{tabular}

\begin{tabular}{|l|l|l|l|l|l|}
\hline 418.6 & 0.49 & 100.00 & 1.156 & 0.00 & 0.00 \\
\hline
\end{tabular}

\begin{tabular}{|l|l|l|l|l|l|l|}
\hline 352.0 & 0.89 & 99.51 & 0.972 & 0.00 & 0.00 \\
\hline
\end{tabular}

\begin{tabular}{|l|l|l|l|l|l|}
\hline 296.0 & 1.70 & 98.62 & 0.818 & 0.00 & 0.00 \\
\hline & 3.39 & 96.92 & 0.688 & 0.00 & 0.00 \\
\hline
\end{tabular}

\begin{tabular}{|l|l|l|l|l|l|l|}
\hline 248.9 & 3.39 & 96.92 & 0.688 & 0.00 & 0.00 \\
\hline
\end{tabular}

\begin{tabular}{|l|l|l|l|l|l|l|}
\hline 209.3 & 6.72 & 93.53 & 0.578 & 0.00 & 0.00 \\
\hline
\end{tabular}

\begin{tabular}{|l|l|l|l|l|l|l|}
\hline 176.0 & 12.04 & 86.81 & 0.486 & 0.00 & 0.00 \\
\hline
\end{tabular}

\begin{tabular}{|l|l|l|l|l|l|}
\hline 176.0 & 12.04 & 86.81 & 0.486 & 0.00 & 0.00 \\
\hline 14.0 & 17.61 & 74.77 & $\mathbf{0 . 4 0 9}$ & $\mathbf{0 . 0 0}$ & $\mathbf{0 . 0 0}$ \\
\hline
\end{tabular}

\begin{tabular}{|l|l|l|l|l|l|l|}
\hline 124.5 & 19.42 & 57.16 & 0.344 & 0.00 & 0.00 \\
\hline
\end{tabular}

\begin{tabular}{|l|l|l|l|l|l|l|}
\hline 104.7 & 16.02 & 37.74 & 0.2890 & 0.00 & 0.00 \\
\hline
\end{tabular}

\begin{tabular}{|l|l|l|l|}
\hline 88.00 & 10.17 & 21.72 \\
\hline
\end{tabular}

\begin{tabular}{|l|l|l|}
\hline 74.00 & 5.47 & 11.55 \\
\hline
\end{tabular}

\begin{tabular}{|l|l|l|}
\hline 62.23 & 2.79 & 6.08 \\
\hline
\end{tabular}

\begin{tabular}{|l|l|l|}
\hline 52.33 & 1.49 & 3.29 \\
\hline
\end{tabular}

\begin{tabular}{|l|l|l|}
\hline 44.00 & 0.87 & 1.80 \\
\hline
\end{tabular}

\begin{tabular}{|l|l|l|}
\hline 37.00 & 0.56 & 0.93 \\
\hline
\end{tabular}

\begin{tabular}{|l|l|l|}
\hline 31.11 & 0.37 & 0.37 \\
\hline
\end{tabular}

\begin{tabular}{|l|l|l|}
\hline 26.16 & 0.00 & 0.00 \\
\hline
\end{tabular}

\begin{tabular}{|l|l|l|}
\hline 22.00 & 0.00 & 0.00 \\
\hline
\end{tabular}

\begin{tabular}{l|l|l|}
\hline 18.50 & 0.00 & 0.00
\end{tabular}

\begin{tabular}{|l|l|l|l|l|}
\hline 15.56 & 0.00 & 0.00 \\
\hline
\end{tabular}

\begin{tabular}{|c|c|c|}
\hline 13.08 & 0.00 & 0.00 \\
\hline 11.00 & 0.00 & 0.00
\end{tabular}

\begin{tabular}{|l|l|l|}
\hline 11.00 & 0.00 & 0.00 \\
\hline 9.25 & 0.00 & 0.00
\end{tabular}

\begin{tabular}{|l|l|l|}
\hline 9.25 & 0.00 & 0.00 \\
\hline 7.78 & 0.09 & 0.00
\end{tabular}

\begin{tabular}{|l|l|l|}
\hline 7.78 & 0.00 & 0.00 \\
\hline 6.54 & 0.00 & 0.00
\end{tabular}

\begin{tabular}{|l|l|l|}
\hline 6.54 & 0.00 & 0.00 \\
\hline 5.50 & 0.00 & 0.00
\end{tabular}

\begin{tabular}{|l|l|l|}
\hline 5.50 & 0.00 & 0.00 \\
\hline 4.62 & 0.00 & 0.00 \\
\hline
\end{tabular}

\begin{tabular}{|l|l|l|}
\hline 4.62 & 0.00 & 0.00 \\
\hline
\end{tabular}

Warnings: NONE

Figure A-0-4. PSD for stainless steel in $\mathbf{5 0} \%$ isopropyl no sonication 
Revision 0

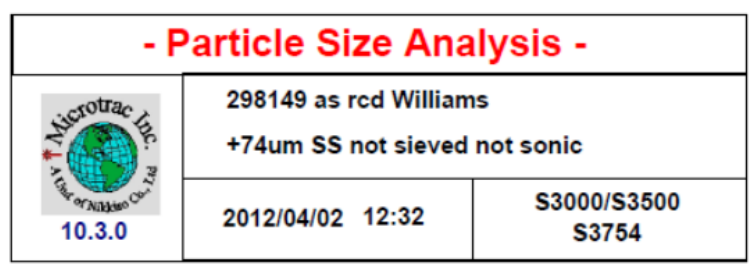

\begin{tabular}{|c|c|}
\hline Data Item & Value \\
\hline MV(um): & 125.4 \\
\hline MN(um): & 70.27 \\
\hline MA(um): & 106.2 \\
\hline CS: & $5.70 E-02$ \\
\hline SD: & 43.65 \\
\hline & \\
\hline
\end{tabular}

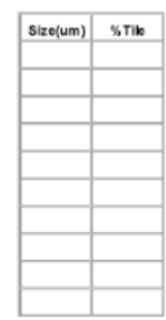

\begin{tabular}{|c|c|}
\hline \%Tile & Size(um) \\
\hline 10.00 & 70.69 \\
\hline 20.00 & 85.45 \\
\hline 25.00 & 91.19 \\
\hline 40.00 & 106.5 \\
\hline 50.00 & 116.5 \\
\hline 60.00 & 127.4 \\
\hline 70.00 & 140.3 \\
\hline 75.00 & 148.2 \\
\hline 90.00 & 188.9 \\
\hline 95.00 & 223.4 \\
\hline
\end{tabular}

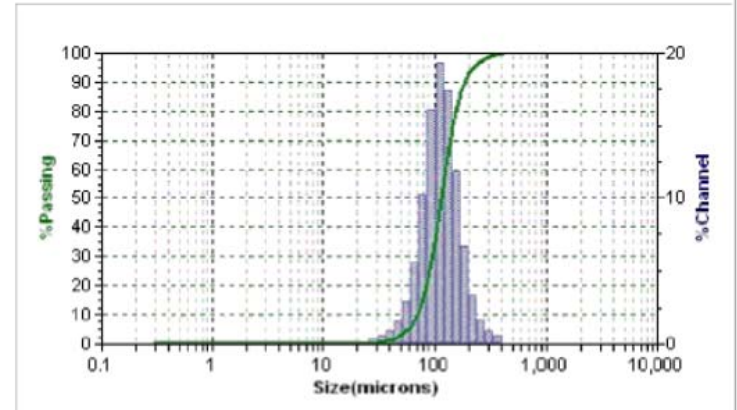

Isopropyl $50 \%$

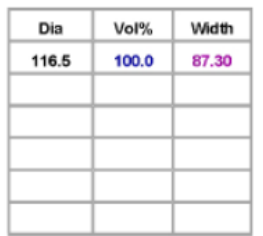

\begin{tabular}{|c|c|c|c|c|c|c|c|}
\hline Distiburer & volume & Run nme: & $30 \sec$ & Flest: & $\begin{array}{l}\text { ISOPROPN } \\
\text { NCOHOL }\end{array}$ & & \\
\hline Mogession & andard & Run Num: & Avo of 3 & Fud Ret index & $w$ & Laadng Fastor: & 0.400 \\
\hline Upper Ecoge: & 1008 & Parrocle: & 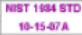 & Above Resisur: & 0 & Transmiseicn: & 0.92 \\
\hline Lament Ecoge & 0243 & Tramsparimey: & Novertiang & Besour Rentuat: & 0 & RMS REHAL: & $4.400-10$ \\
\hline Resicunas: & Ensoled & Pat ket, ndex & WA & & & pros: & $\operatorname{sos}$ \\
\hline Mati. Charrels: & so & Part shape: & inregatar & $\operatorname{ces} 10:$ & 0505 & Usodic Pomer: & neA \\
\hline Maxysus thode: & 50000 & & & & & Unade Then: & nia \\
\hline Filer: & Ensuled & De Recard: & n:so & Recalo Oatunn: & & Serid Mum: & $\$ 73$ \\
\hline
\end{tabular}

\begin{tabular}{|c|c|c|c|c|c|c|c|c|c|c|c|}
\hline Sizelum) & WChan & W Pass & Size(um) & WChan & W Pass & Sizefum) & NChan & * Pass & Sizolum) & KChan & * Pass \\
\hline
\end{tabular} \begin{tabular}{|l|l|l|l|l|l|l|}
\hline 1408 & 0.00 & 100.00 & 3.89 & 0.00 & 0.00 \\
\hline 1184 & 0.00 & 100.00 & 3.27 & 0.00 & 0.00 \\
\hline
\end{tabular}

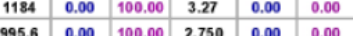

\begin{tabular}{|l|l|l|l|l|l|l|l|}
\hline 95.0 .00 & 0.00 & 100.00 & 2750 & 0.00 & 0.00 \\
\hline
\end{tabular}

\begin{tabular}{|l|l|l|l|l|l|l|}
\hline 857.2 & 0.00 & 0.00 & 2.312 & 0.00 & 0.00 \\
\hline
\end{tabular}

\begin{tabular}{|l|l|l|l|l|l|l|}
\hline 704.0 & 0.00 & 100.00 & 1.945 & 0.00 & 0.00 \\
\hline 592.0 & 0.00 & 100.00 & 1.035 & 0.09 & 0.00 \\
\hline
\end{tabular}

\begin{tabular}{|l|l|l|l|l|l|}
\hline 592.0 & 0.00 & 100.00 & $\mathbf{1 . 6 3 5}$ & 0.00 & 0.00 \\
\hline & 0.00 & 100.00 & 1.355 & 0.09 & 0.00 \\
\hline
\end{tabular}

\begin{tabular}{|l|l|l|l|l|l|}
\hline $\mathbf{4 9 7 . 8}$ & $\mathbf{0 . 0 0}$ & 100.00 & $\mathbf{1 . 3 7 5}$ & $\mathbf{0 . 0 0}$ & 0.00 \\
\hline 418.6 & 0.51 & 100.00 & $\mathbf{1 . 1 5 6}$ & 0.00 & 0.00 \\
\hline 352.0 & 0.91 & 99.49 & 0.972 & 0.00 & 0.00 \\
\hline
\end{tabular}

\begin{tabular}{|l|l|l|l|l|l|l|}
\hline 418.6 & 0.51 & 100.00 & 1.156 & 0.00 & 0.00 \\
\hline
\end{tabular}

\begin{tabular}{|l|l|l|l|l|l|l|}
\hline 352.0 & 0.91 & 99.49 & 0.972 & 0.00 & 0.00 \\
\hline
\end{tabular}

\begin{tabular}{|l|l|l|l|l|l|}
\hline 296.0 & 1.72 & 98.58 & 0.818 & 0.00 & 0.00 \\
\hline
\end{tabular}

\begin{tabular}{|l|l|l|l|l|l|}
\hline 248.9 & 3.40 & 96.86 & 0.688 & 0.00 & 0.00 \\
\hline
\end{tabular}

\begin{tabular}{|l|l|l|l|l|l|}
\hline 209.3 & 6.66 & 93.46 & 0.578 & 0.00 & 0.00 \\
\hline & 1189 & 06 & 0.486 & 0.00 & 0.00 \\
\hline
\end{tabular}

\begin{tabular}{|l|l|l|l|l|l|l|}
\hline 176.0 & 11.89 & 86.80 & 0.486 & 0.00 & 0.00 \\
\hline
\end{tabular}

\begin{tabular}{|l|l|l|l|l|l|l|}
\hline 148.0 & $17 A 2$ & 74.91 & 0.409 & 0.00 & 0.00 \\
\hline
\end{tabular}

\begin{tabular}{|l|l|l|l|l|l|l|}
\hline 124.5 & 19.32 & 57.49 & 0.344 & 0.00 & 0.00 \\
\hline
\end{tabular}

\begin{tabular}{|l|l|l|l|l|l|l|}
\hline 104.7 & 16.05 & 38.17 & 0.2890 & 0.00 & 0.00 \\
\hline
\end{tabular}

\begin{tabular}{|l|l|l|}
\hline 88.00 & 10.28 & 22.12 \\
\hline 85.00 & 5.55 & 11.04
\end{tabular}

\begin{tabular}{|l|l|l|}
\hline 74.00 & 5.58 & 11.84 \\
\hline
\end{tabular}

\begin{tabular}{|l|l|l|}
\hline 62.23 & 2.87 & 6.26 \\
\hline
\end{tabular}

\begin{tabular}{|l|l|l|}
\hline 52.33 & 1.54 & 3.39 \\
\hline
\end{tabular}

\begin{tabular}{|l|l|l|}
\hline 44.00 & 0.90 & 1.85 \\
\hline
\end{tabular}

\begin{tabular}{|l|l|l|}
\hline 37.00 & 0.57 & 0.95 \\
\hline
\end{tabular}

\begin{tabular}{|l|l|l|}
\hline 31.11 & 0.38 & 0.38 \\
\hline
\end{tabular}

\begin{tabular}{|l|l|l|}
\hline 26.16 & 0.00 & 0.00 \\
\hline
\end{tabular}

\begin{tabular}{|l|l|l|l|}
\hline 22.00 & 0.00 & 0.00 \\
\hline
\end{tabular}

\begin{tabular}{|l|l|l|}
\hline 18.50 & 0.00 & 0.00 \\
\hline 15.56 & 0.00 & 0.00
\end{tabular}

\begin{tabular}{|l|l|l|l|}
\hline 15.56 & 0.00 & 0.00 \\
\hline 13.08 & 0.00 & 0.00
\end{tabular}

\begin{tabular}{|l|l|l|l|l|l}
\hline 13.08 & 0.00 & 0.00
\end{tabular}

\begin{tabular}{|c|c|c|}
\hline 11.00 & 0.00 & 0.00 \\
\hline 9.25 & 0.00 & 0.00
\end{tabular}

\begin{tabular}{|l|l|l|l|}
\hline 9.25 & 0.00 & 0.00 \\
\hline 7.00 & 0.00 & 0.00
\end{tabular}

\begin{tabular}{|l|l|l|l|l|}
\hline 7.78 & 0.00 & 0.00 \\
\hline 6.54 & 0.00 & 0.00 &
\end{tabular}

\begin{tabular}{l|l|l|l|}
\hline 6.54 & 0.00 & 0.00 \\
\hline 5.50 & 0.00 & 0.00
\end{tabular}

\begin{tabular}{|l|l|l|}
\hline 6.54 & 0.00 & 0.00 \\
\hline 5.50 & 0.00 & 0.00 \\
\hline 4.62 & 0.00 & 0.00 \\
\hline
\end{tabular}

\begin{tabular}{|l|l|l|}
\hline 4.62 & 0.00 & 0.00 \\
\hline
\end{tabular}

Warnings: NONE

Figure A-0-5. PSD for stainless steel in $\mathbf{5 0 \%}$ isopropyl and with sonication 
Revision 0

\section{Appendix B: Test Direction}


[Note: This Direction indicate Campaign 2 but they were the same for Campaign 1 except jet pump flowrates which changed from $8 \mathrm{gpm}$ to $8.4 \mathrm{gpm}$ and this reduced the mixing times in the MDT; therefore, the table listing the times have been changed.]

R\&D Directions: Fill and Empty, Rev. 2 - $\underline{\text { Campaign } 2}$ - 13 June 2012

Author: M.R. Duignan, Principal Investigator (PI)

Reviewer : J.L. Steimke

\section{Terminology}

1. $\quad$ ADS = Analytical Development Section (of SRNL)

2. $\quad \mathrm{DAS}=$ Data Acquisition System

3. $\mathrm{FM}=$ Flowmeter

4. $\quad \mathrm{FT}=$ Fill Feed Tank

5. $\mathrm{JP}=$ Jet Pump

6. $\mathrm{MDT}=$ Mixing Demonstration Tank

7. $\mathrm{MS}=$ Magnetic Separator

8. $\quad \mathrm{PP}=$ Peristaltic Pump

9. $\quad \mathrm{RT}=$ Receipt Tank

10. SASS $=$ Solids Accumulation Scouting Studies

11. $\mathrm{SS}=$ Stainless Steel

12. $\mathrm{VFD}=$ Variable Frequency Drive (for the pumps)

13. 1 Cycle Batch is a full MDT 18.7-inch MDT Slurry Height from tank bottom

14. 1 Cycle Batch contains 7 Transfer Batches and 1 Heel

15. Transfer Batch $=\mathrm{TB} \sim 2.4$-inch MDT slurry height between TBs

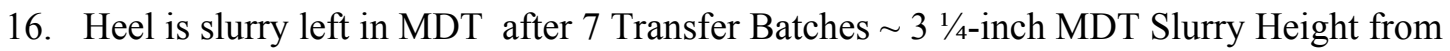
tank bottom

17. Supernate is used instead of supernatant

\section{Overall Campaign Operational Sections:}

1. Fill FT

2. Fill MDT

3. Mix MDT - Prepare for TBs

4. MDT Batch Transfers

5. Remove SS from Transferred Batch

6. Flush RTs

7. Preparation for Heel Measurements

\section{Assumptions}

1. Test personnel are familiar with appropriate eHAPs (SASS, 786-5A, etc)

2. Proper PPE is available (lab coat, safety glasses with side shields, and disposable gloves when handling simulant and taking samples.

3. Test supplies are on hand (four solids, supernatant, sample materials, etc.) 
4. FT, MDT, and each RT are calibrated for volumes.

5. Electronic/Computer equipment is turned on and ready for use.

6. DAS will continuously acquire data through a day's operation at a frequency indicated by the PI.

7. Fractional Crystallization totes Nos. $3 \& 4$ are close by and ready for use. The supernatant in both totes has been sampled for the full suite of ADS analyses to baseline the test material.

\section{Actions}

1. All hand measurements shall be entered into the task notebook.

2. All measurement equipment shall be noted in the task notebook along with control number to obtain calibration information when needed.

3. PI will supply the FT feed rate, Jet Pump flow rate, and the Jet Pump rotational speed.

\section{Solids Concentrations to Fill FT:}

Cycle $1 \mathrm{FT}$ volume $=392.24$ liters

Included Solids $=100$ grams $/$ liter

Gibbsite: $\quad 27.849 \mathrm{~kg}$

ZrO2: $\quad 5.884 \mathrm{~kg}$

Yellow Sand: $5.099 \mathrm{~kg}$

Stainless Steel: $0.392 \mathrm{~kg}$

Total Mass $=\quad 39.224 \mathrm{~kg}$
All other cycles $=324.35$ liters

Gibbsite: $\quad 23.029 \mathrm{~kg}$

ZrO2: $\quad 4.865 \mathrm{~kg}$

Yellow Sand: $4.217 \mathrm{~kg}$

Stainless Steel: $0.324 \mathrm{~kg}$

Total Mass $=32.435 \mathrm{Kg}$

\section{Fill Feed Tank}

1. Shut all valves on the FT system.

2. Fill FT with supernatant to the fill marks with approximately 300 liters ( $\sim 80$ gal) if Cycle 1 or 250 liters ( 66 gal.) for all other cycles. Note, for Cycles 1 and 2 of Campaign 1 the supernatant will come from Fractional Crystallization totes Nos. 3 and 4. For all other cycles the supernatant will be decanted from the four settling drums to reuse previously processed supernatant. For example, for Cycle 3 of Campaign 1 the supernatant used for Cycle 1 should be decanted from the settling drums through the FT filter. Fresh supernatant is only used to make up for losses during operation.

3. Open valves FT1, FT2, and FT4.

4. Turn FT3 to FT.

5. Start Pump 4 to begin circulating liquid by opening air valve FT11.

6. Start FT agitator at VFD and increase speed until simulant appears to be mixing well.

7. Slowly pour, or scoop, in the gibbsite needed. If necessary, cover the FT with plastic to prevent powder dispersion.

8. Repeat Step 7 for the $\mathrm{ZrO} 2$.

9. Repeat Step 7 for the Sand.

10. Have ready SS, but only pour it directly into the FT when ready to transfer to the MDT.

11. The FT is now ready for transfer when the MDT is ready for a new cycle batch.

\section{Feed Tank Filled}




\section{Fill Mixing Demonstration Tank}

2. If the MDT is ready and empty, then shut drain valve at bottom of the MDT, otherwise the MDT has a heel of slurry.

3. Begin the DAS at a frequency indicated by the PI to record FM 4.

4. Pour into the FT the SS and allow mixing for a few minutes.

5. Open valve FT9 and then turn FT3 to the MDT to begin transferring the new batch to the MDT at approximately $2 \mathrm{gpm}$ (or flowrate determined during trial test runs).

6. As the FT level drops and exposes the agitator the speed can be reduced and then stopped when the agitator is fully exposed.

7. When the FT system transferred all the slurry possible through valve FT3 it may be necessary to first slow pump 4 to allow the suction line at the bottom of the FT to remain full. When the slurry is completely in the suction line and the flow to the MDT slows then note the MDT slurry level, which needs to be increased to 18.7 inches - A full cycle batch.

8. Either pour 15 to 20 liters of fresh supernatant directly into the FT or decant supernatant from the settling drums but opening valve FT6, closing FT4 to flush the FT and top off the MDT.

9. Circulate the 15 to 20 liters in the FT system while simultaneously filling the MDT.

10. Repeat Steps 18 to 20 as needed until the MDT is filled, then turn FT3 to the FT and close FT9 to stop flow to the MDT.

11. Shut air valve FT-11 to stop pump 4 .

12. Once the FT is empty the next charge of simulant can prepared to be ready for the following cycle. (Note the FT does not have to be empty and supernatant used for flushing that remains can be used for the next batch) Repeat Steps 1 to 12, which can be done before or after test begins and then Steps 13 to 22 .

\section{MDT Filled and Ready}

\section{Mix MDT - Prepare for Transfer Batches}

13. Prepare to start the slurry pump that feeds the two jet pumps: Turn on the pump seal water system to the slurry pump:

a. Turn on the house water to circulate cooling water through the pump seal water reservoir cooling coil.

b. If not already open, then open the pump seal water valves to allow the seal water to circulate.

c. Turn on the seal water pump. The seal water pressure needs to be at least 15 psi over the system pressure of the slurry pump, pump 1 . For Campaign 1 pump 1 will be operating at $16 \mathrm{gpm}$ which results in a pressure of approximately $9.5 \mathrm{psig}$. The seal pump pressure should be set to at least 25 psig. The seal water flow rate can be set at 2 gpm.

Caution: Never deadhead the slurry pump unless the seal water is above 50 psig because the slurry pump can go to 30 psig and lower seal water pressures will cause slurry to leak into the pump seal and cause damage.

d. Turn the seal water ball valve to adjust the flowrate through the seal water rotameter such that the indicator is not at its maximum measureable flowrate. 
e. Adjust the seal water rotameter valve obtain a flow of about $2 \mathrm{gpm}$.

14. Open valves V1, V2, V3, V4. Close V5 and V6.

15. If not already done, turn on DAS with a data frequency indicated by the PI.

16. Start the slurry pump VFD and adjust the frequency to about $10 \mathrm{~Hz}$ to obtain a low flowrate of 1 to 3 gpm.

17. Begin rotating jet pump nozzles at speed specified by PI.

Notes

a. The jet of each pump shall to be synchronized so that they meet at the center of the tank on each rotation. That is, each time one of the nozzles for each pump is oriented at the center the nozzle of the opposing jet pump shall be also pointing at the tank center.

b. Pumps shall not be rotated without some slurry flow through jet pumps.

18. Slowly increase the pump VFD frequency. For Campaign 1 the frequency is

approximately $29 \mathrm{~Hz}$, to obtain 8 gpm/jet pump, for Campaign 2 the PI will indicate new values.

19. Allow the MDT to mix until the volume is recirculated five (5) times. Campaign 2 will use a nozzle jet velocity of $23.5 \mathrm{ft} / \mathrm{s}$, that is, each jet pump will have a flowrate of 8.4 $\mathrm{gpm}$, or $16.8 \mathrm{gpm}$ for both jets. The minimum mixing times for $5 \mathrm{x}$ MDT volume times shall be:

Batch Transfer 1 Tank Volume 103.6 gal x $5=518.1$ gal needing: Batch Transfer 2 Tank Volume 90.5 gal x $5=452.4$ gal needing: Batch Transfer 3 Tank Volume 77.4 gal x $5=386.8$ gal needing: Batch Transfer 4 Tank Volume 64.2 gal x $5=321.1$ gal needing: Batch Transfer 5 Tank Volume 51.1 gal x $5=255.4$ gal needing: Batch Transfer 6 Tank Volume 38.0 gal x $5=189.8$ gal needing: Batch Transfer 7 Tank Volume 24.8 gal x $5=124.1$ gal needing:

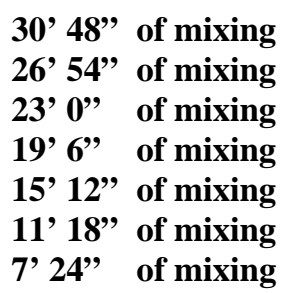

20. During mixing take note of the action and movement of the solids, especially those on the bottom of the tank. Rough measurements of final size and placement near the end of the time period should be noted. This information shall be included in the task notebook.

21. During the MDT mixing prepare to transfer a batch to the RTs: All valves of the RT system should begin closed.

22. Just before transferring to a receipt tank take photographs of the accumulated solids on the bottom of the tank and measure the length and width either directly or having a measure on the bottom so it is recorded in the photographs.

\section{MDT Mixed and Ready for Transfer}

\section{MDT Batch Transfers}

23. At the start of a day pump 3 will be filled with supernatant to prevent solids plugs.

24. All Magnetic Separator (MS) valves should be closed.

25. Begin recirculation through pump 3 by:

a. Turn V19 to recirculation tube (tube between V19 and V24).

b. Turn V7 to FM 3.

c. Close valves V20, V25, and open V24.

d. Turn V14 to V24. 
e. Turn on pump 3 to circulate supernatant.

f. If circulation does not begin check tubes and pump for plugs.

26. When a TB is to begin turn off pump 3 to stop the recirculation.

27. Close V24 and open V20 and V25.

28. Turn V19 and V8 to transfer tube.

29. Turn V15 to RT header.

30. Open valve to RTx, that is, $\mathrm{Vx} 1$ for $\mathrm{RTx}$, where $\mathrm{x}=1$ to 7 .

31. Immediately turn on pump 3 to begin transfer.

32. If flow does not start check tubes and pump for plugs:

a. Lightly tap tube from FM 3 to V14.

b. Lightly tap the discharge and suction ports of pump 3 .

c. Check to see if any plug is in the MS and if so valves V20 and V25 can be used to flow through the MS channels separately.

d. Using the pressure head of the clean supernatant reservoir flow through the MS can be checked both in forward and backward flow.

e. There are many possible combinations that can be tried until the system is free of plugs.

f. As a last resort the MS system can be flushed with pressurized water.

33. Immediately adjust pump 3 speed to attain flow rate on FM 3 of 0.58 gpm

34. Wait for approximately 15 minutes for RTs $1-6$ or 6 minutes for RT 7 because it take several more minutes for the RT to finish filling. That is, it takes approximately $221 / 2$ minutes to fill each of the first six (6) RTs and 12 minutes to fill RT 7.

35. During each transfer document in the task notebook any observations of solids movements in both the MDT and the RTs. Note, the shape and height of the MDT solids mounds if possible and take photographic evidence from the top, sides and bottom, when useful.

36. When the wait time is finished, closely watch the fill line on the appropriate RT.

37. Stop the TB by shutting pump 3.

38. Immediately shut the appropriate RT feed, that is, valve Vx1.

39. As soon as possible, stop the jet pump rotation and then turn off pump 1 to stop MDT mixing by reducing the VFD to $0 \mathrm{~Hz}$ and then STOP, then immediately close valves V1, $\mathrm{V} 2, \mathrm{~V} 3$, and V4.

40. Wait exactly 20 minutes and then record the sand height in RTx and if possible the $\mathrm{ZrO} 2$ height.

\section{MDT Batch Transfer Complete}

\section{Remove SS from Transferred Batch}

Note: During the 20-minute wait after a TB the SS needs to be removed from the MS.

41. As soon as TB flow is stopped prepare to remove SS solids from the MS.

42. Fill the clean supernatant reservoir to the MS with supernatant, if not done already.

43. Place 3-liter container under purge drain of the MS.

44. Turn V15 to V16, then turn V14 to V17 and V17 to connect Clean Supernatant tank to flush non-SS solids. 
45. Turn V16 to purge drain and close V17 when approximately 1 liter collected. Dump this material into empty settling drums.

46. Carefully remove the magnet holder and store the magnetic holder in plastic pipe holder provided. (KEEP IT AWAY FROM MAGNETIC MATERIALS). Quickly replace with non-magnetic blank.

Caution: Never remove magnetic holder or blank when it resists retraction because there is pressure on the system. Forcing the holder out will damage the MS.

47. Place sample container (3-liters container) under purge drain.

48. Open V17 to supernatant reservoir to begin the SS purge.

49. If solids appear to be caught up in some MS locations try tapping the MS gently and giggle the non-magnetic blank. Another method is to remove any pressure off the magnetic blank, then gently remove the blank and replacing it with the magnetic holder, then removing the magnetic holder and replacing it with the blank once more. This should dislodge any remaining SS solids.

50. After all the SS solids are purged (it may take several liters and toggling V16 closed and open several times) close V17.

51. Replace non-magnetic blank with the magnetic holder.

52. Turn V16 to clean supernatant.

53. Turn V17 to purge drain to refill MS with supernatant.

54. Close V17.

55. Turn V15 to RT header.

56. Turn V14 to FM 3.

57. The MS in now ready for the next transfer.

58. If another TB will follow put the MS system in recirculation mode.

a. Turn V19 to the recirculation line.

b. Close V20 and V25, open V24.

c. Turn on pump 3.

59. If another TB will not follow the flush the MS system:

a. Turn V7 to drain.

b. Turn V19 to the recirculation line.

c. Close V20 and V25, open V24.

d. Turn V14 to V17.

e. Turn V17 to clean supernatant.

f. Turn on pump 3.

g. Allow the supernatant to flush pump 3, the stop pump 3.

h. Return MS system to be ready for next transfer:

- Close V17 and V24.

- Open V20 and V25.

- Turn V19 to transfer line.

- Turn V14 to MS.

- Turn V7 to FM 3.

60. The SS can be now removed from the catch container with the hand-held magnet and placed in a sample bottle to measure. 
61. When the 20 minutes of settling time have elapsed record the solids heights in the appropriate RT.

62. Go to Section 3 to begin the process of mixing MDT if another transfer is to me made.

63. If this is the $7^{\text {th }}$ Transfer and Heel Measurement are to be done immediately go to Section 7, otherwise, go to Section 6 to empty the RTs.

\section{SS is Now Removed and Ready for the Next Transfer}

\section{Flush Receipt Tanks}

64. The seven RTs may be emptied immediately after each filling, but the PI may want to empty them only after all seven are filled. This will be decided on a Cycle by Cycle basis. However the process of emptying is the same.

65. Verify the air header supplying air to the RTs is pressurized to $\sim 100 \mathrm{psig}$.

Caution: The air supply to the RT should be off at all times when not flushing the RTs by maintaining valve V9 closed. As such, air will not be leaking into any of the RTs because one of the seven individual air valves to each $R T$ was accidentally left open or leaks. Open V9 only when RT flushing is to begin.

66. Verify the slurry transfer line leading to one of the four settling drums is secured in the drum. Note, all seven RTs, for a single cycle, have 86 gallons of volume, therefore, both 55-gal drums to be used shall have at least $45 \mathrm{gal}$ of space each before the first $R T$ is transfer. That is, if there are settled solids already in the drums those solids cannot occupy more than $10 \mathrm{gal}$ of space. This shall be verified before a series of RT emptying begin. The settled solids in the drums can be emptied from the drums at any time after the supernatant is decanted, but they shall not be allowed to occupy more than 10 gal of space that would prevent their use for the next cycle of operation.

67. Open V9 and begin emptying an RT slowly by opening air valve Vx3, where $x=1$ to 7 , just enough to suspend the settled solids.

68. When the solids are suspended open pump 2 suction line valve V18.

69. Open $\mathrm{RT}$ drain valve $\mathrm{Vx} 2$. Where $\mathrm{x}=1$ to 7 .

70. Open V10 which supplies air to pump 2 to begin pumping slurry out of the RT to fill the settling drums.

71. When the emptying RT begins to flow smoothly the air valve can be closed, but it can be opened periodically if solids begins to plug.

72. While one RT is emptying open the air valve on the next RT to be emptied to begin mixing the contents.

73. When the RT is empty open the next drain valve Vx2. Where $\mathrm{x}=1$ to 7 and close the one that was open.

74. Repeat Steps 83 to 84 until all seven RTs are empty, but carefully monitor the settling drums so they are not over filled.

Note: The sight glass on RT 1 to RT 6 can plug with solids during use. To prevent plugging a small amount of supernatant can be poured (squirted) down inside the sight glass when the simulant level drops below the glass.

75. Before shutting pump 2 reopen drain valves $V x 2$ one at time from 1 to 7 to further empty the drain line and allow pump 2 to fully empty into the settling drums.

76. Finally, shut all valves Vx2 and air valve V10 to stop pump 2, and close V18.

77. Close the air header valve V9. 


\section{RTs Are Ready for Next Campaign Cycle}

\section{Preparation for Heel Measurements}

Note: This direction assumes the MDT is at the heel level of $31 / 4$ inches and mixing is stopped from after a $7^{\text {th }}$ batch transfer.

78. If solids have settled start jet pumps (JPs) and rotation as if at the end of the $7^{\text {th }}$ transfer:

a. Start JPs slow ( $<3 \mathrm{gpm} /$ pump, VFD to $\sim 10 \mathrm{~Hz}$ ).

b. Start rotation at $1.57 \mathrm{rpm}($ Voltage $=3.58)$.

c. Increase JP flow to $8 \mathrm{gpm}(\mathrm{VFD}=29 \mathrm{~Hz})$

d. Allow the mixing to continue until all the solids that settled from $7^{\text {th }}$ transfer are suspended and the movement of solids from below appears similar to what it was before the mixing was stopped.

79. Take photos of the accumulated solids on the bottom and the tank side if there are visible solids.

80. Stop JP rotation when it is at $0^{\circ}$ or $180^{\circ}$.

81. Reduce JP flow rate to $<3 \mathrm{gpm}(\mathrm{VFD} \sim 10 \mathrm{~Hz})$.

82. With a peristaltic pump (PP) connected to the transfer line begin draining down the heel in a container that can hold at least 17 gallons. Flow rate can be the maximum the available PP can transfer $(\sim 2.9 \mathrm{lpm})$.

83. Make note of the tank heights when peaks of the accumulated solids appear.

84. As the heel level drops carefully monitor the exposed solids. The JP circulation should be just enough to keep the gibbsite/zirconium solids suspended but not enough to disturb the already settled solids. To do this the JP VFD frequency can be reduced from 10 to 8 to $6 \mathrm{~Hz}$, etc.

85. When the heel drops to the point of exposing the jets then stop the JP mixing.

86. Allow the PP to reduce the heel level until the transfer line just begins to drawn air, then stop the PP. This level is at about $1 / 4$ inch.

87. Place a container under the MDT drain line and open the MDT central bottom drain to continue emptying the MDT.

88. While the final draining is proceeding, place the Laser Positioning System on the tank, if not already in place.

89. When the draining stops start a laser scan of the tank bottom. (About 1 hour.)

90. When the laser scan is complete sample the core on the North mound. Sampling location will depend on the mound but choose at least four locations.

91. When core sampling is complete close the MDT bottom drain until it is flush with the tank bottom.

92. When complete take a photograph of the accumulated solids from the top and bottom of the MDT.

93. Pour the container with what came out of the bottom drain into the PP hold tank that has the rest of the heel slurry.

94. After core sampling is complete reverse the PP pump flow direction to begin slowly refilling the heel.

95. At approximately $1 / 4$-inch intervals stop the heel filling to photograph the exposed accumulated solids. 
96. Continue Steps 105 and 106 until the accumulated solids are completely submerged, then continue replacing the heel until all heel material has been returned and the heel level is at its starting height, about $3 \frac{1 / 4}{4} \mathrm{inch}$.

\section{End of Direction - MDT is ready for the next Cycle}


Revision 0

Appendix C: Lessons Learned 


\section{Pump Issues}

There were several issues with pumps used in this study. The final simulant chosen for the study has considerable solids including gibbsite, zirconia, sand, and chopped SS wires. The gibbsite and zirconia are on the order of $8 \mu \mathrm{m}$ in diameter. They tend to work themselves through the seals. They also appear to adhere to themselves and other materials. The sand and SS are on the order of $100 \mu \mathrm{m}$, are abrasive, and tend to settle out quickly in low flow regions or especially when flow is stopped.

For a proof test of the magnetic separator we attempted to use a common SS centrifugal pump with a typical alumina/graphite mechanical seal. We knew that pump was not the best choice for pumping solids, but had considerable experience using one for similar simulants. The test was to be only about 30 minutes long, far shorter than we would have expected problems to occur based on previous experience. However, the discharge valve was accidentally closed by a hose dragged across the handle. Within seconds the seal failed.

The primary pump issue revolved around pump selection for the transfer of simulant from the MDT through the magnetic separator to the Receipt Tanks. In previous Hanford mixing studies we had used a Moyno 500 series progressive cavity pump as shown in Figure C-0-1. That pump is a relatively positive displacement pump designed to handle slurries of high solids content. It has a SS corkscrew shaped rotor that turns inside a stationary elastomeric stator. Our pump has a ceramic/graphite mechanical seal to prevent leaks to ambient. Before starting this current study, we took the pump apart to replace the seal and examine it for excessive wear. We were surprised to find the pump contained an appreciable amount of SS from the previous testing. This raised concerns since determining the amount of SS transferred with the other solids was a key part of our planned study. It is obvious the pump has a relative large area where solids can accumulate both on the suction and discharge side. Since the suction and discharge are normally on the top of the pump, fast settling solids are most likely to accumulate.

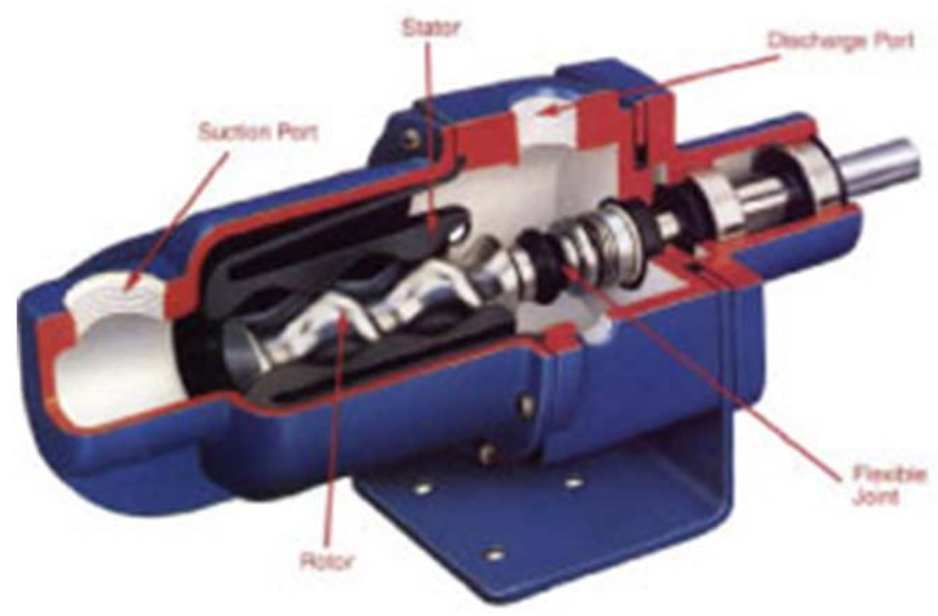

Figure C-0-1 Moyno Pump Used (from Moyno.com Website).

We considered the use of other pumps. The transfer pump must be able to lift simulant out of the tank at the start of each transfer. That ruled out a centrifugal pump by itself since they will not pump until they are primed. We didn't want the added complication of a priming pump ahead of the centrifugal pump. We tried a peristaltic pump but there was too much pressure drop through the transfer piping and magnetic separator, especially since the pump also needed to generate sufficient head to fill up the Receipt Tanks to several feet of height. We considered an air operated double diaphragm pump, but were concerned that the strong pulsation would cause 
problems in the magnetic separator, and likely have non-prototypical effects in the MDT at the transfer line inlet.

We finally decided that the Moyno pump could be used if it were mounted upside down with the suction and discharge pointing downward. That would have the effect of causing the fast settling solids to leave the pump rather than accumulate in the pump cavities. It was expected that some buildup might occur, but would rapidly reach a steady state and hence not affect the test significantly.

The prime mover for the jet mixers is a Galigher 1.5VRA 1000 pump designed to handle slurries with very large solids. It has a rubber lined impeller and housing. The impeller has vanes on the back side to help keep solids away from the seal. The seal on this particular pump consists of double ceramic/graphite mechanical seals with water between them pressurized to about $15 \mathrm{psi}$ above the process fluid pressure. We have had very good experience using this pump with a wide variety of slurries on other studies. It performed fairly well for this study, but we did have to replace a seal that wore down to the point that the seal load spring failed to maintain sufficient pressure to keep a nominally stationary o-ring from spinning and tearing to pieces. We also had problems with the fine solids working their way into the seal water, especially when the pump is shut down and the seal water pump stopped. We eliminated many problems by keeping the seal water running when the Galigher pump was shut down between transfers, and closing the suction and discharge valves on the jet mixer piping when the pump was shut down.

\section{Jet Mixer Issues}

We had considerable difficulty with the jet mixers due to accumulated wear from previous testing. These problems were not unexpected due to their history. The mixers were originally designed for a three week long test about three years ago; therefore their longevity was not emphasized. Rather, the design focused on the ability to build them at minimum cost and in a short time to meet the original test schedule constraints. However, the design proved to be fairly robust and the mixers have been operated for many more hours than originally envisioned, sometimes with some rather challenging simulants. They are simply wearing out now.

Figure C-0-2 shows the current design of the jet mixers. Slurry is sucked out of the tank through the central tube by the Galigher pump. It is pumped back to the mixer and flows down the annular tube and out the two nozzles at the bottom. Since the mixer rotates, both the suction and discharge have to pass through slots in the rotating tubes from the stationary housing. The suction and discharge chambers are separated by an o-ring seal at the top of the central suction tube. There are three o-ring seals between the rotating portion of the mixers and the stationary housing. When the lower o-ring wears, some of the pumped slurry leaks out and drips down into the tank. Unless the leak gets too large it does not cause any appreciable problem. When the middle o-ring wears, some of the pumped slurry leaks through to the suction side rather than exit through the nozzles. There must be considerable wear before the bypass becomes excessive. One problem, however, is that bypassing is difficult to recognize and even harder to quantify. At one point during the current testing, the middle o-ring was accidentally displaced out of the groove during reassembly. The problem was quickly discovered since the loss of flow out the nozzles was dramatic. The major problem with the current design of the jet mixers is due to wear of the top seal o-ring. As the seal wears, air starts to be sucked in by the Galigher pump. Initially the air just leads to foaming in the slurry, and possibly errors in the magnetic flowmeters which have to have good electrical contact with the slurry inside the flow channel. Eventually, the Galigher loses its prime due to the air and stops pumping. 


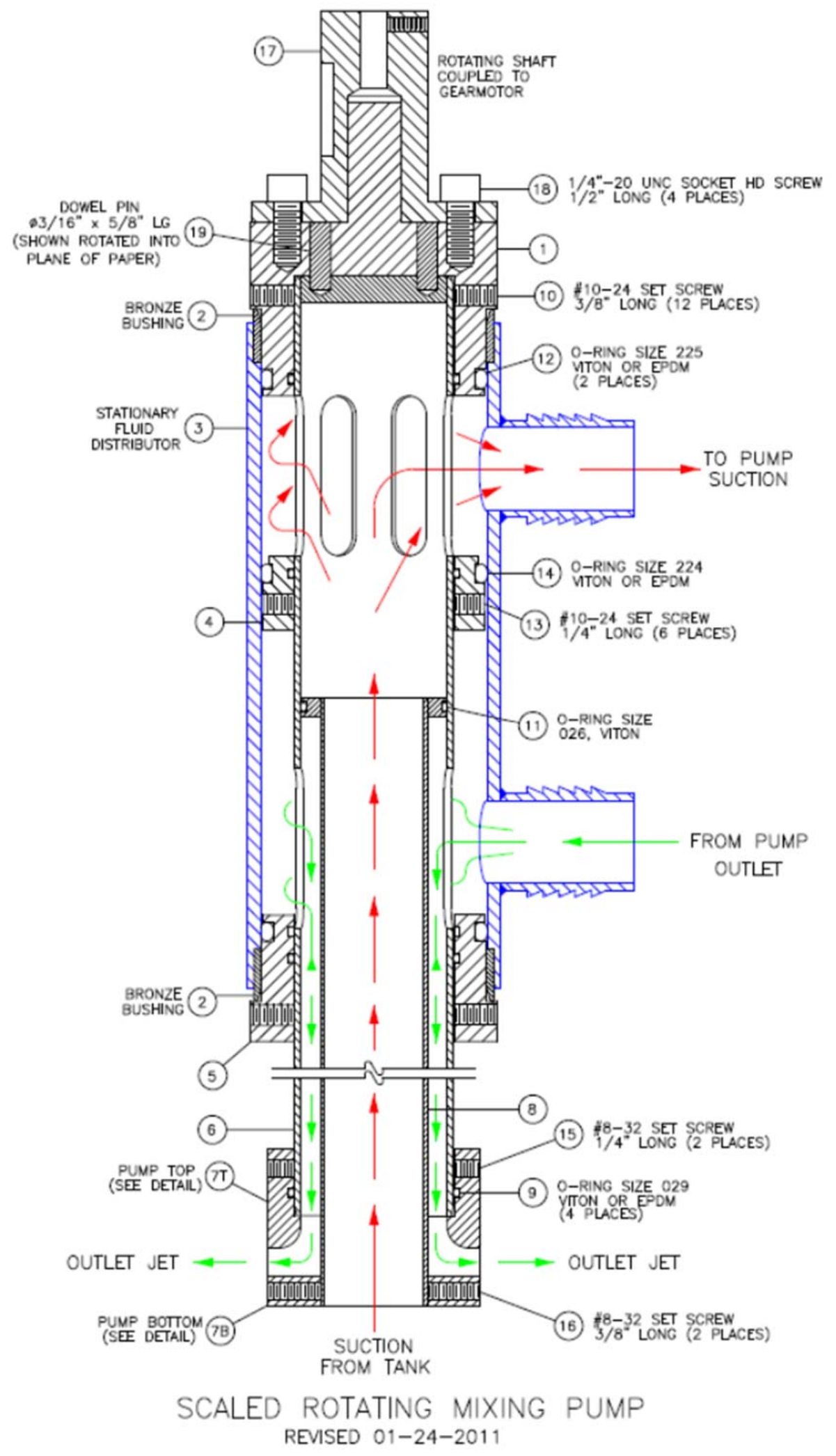

Figure C-0-2. Jet pump - original design. 
In the original design, Teflon O-rings were used as both the seals and the radial bushings. Teflon thrust bushings were also provided. Fairly quickly, the design was improved by providing oilite bronze bushings to handle both radial and thrust loads. The seals were changed to rubber (viton or EPDM) o-rings. Since these were quick, low cost retrofits to the original design for another short term test, the bushings could not be made very large. Therefore they wear faster than desirable. When they wear there is additional compression applied to one side of the o-rings causing higher normal forces, high resistance to rotation, and excessive wear of the o-ring. At this time the bushings are badly worn and should be replaced.

A redesign of the jet mixers was completed early in preparation for this study, Figure C-0-3, and new mixers were to be fabricated. However, additional costs associated with significant simulant changes and schedule acceleration caused the fabrication of improved mixers to be deferred. Again, the testing was fairly short and it was expected that the mixers would last long enough. That appears to be the case, but we have had to replace the o-rings several times during the test.

The major change is to eliminate the rotation on the suction side by passing a stationary tube down through the inner rotating tube. The only rotating seals then needed are on the discharge side which is pressurized. Wear of those seals will just lead to drips into the tank, and have little significance unless the leaks get large. The rotating seal between the suction and discharge chambers of the previous design is also completely eliminated, so there is no possibility of bypassing slurry between the suction and the discharge as before. The new design has fewer rotating seals and they are on the inner side of the chamber, so there should be less friction making the mixer easier to rotate. The bushings are larger so there should be less wear there as well. Finally, a secondary Teflon seal has been added to keep the solids away from the primary o-ring seals, which should extend their life.

\section{Pluggage Problems}

We had several problems with plugs in the small diameter tubing used for the transfers from the MDT through the magnetic separator to the Receipt Tanks. Figure C-0-4 shows the original planned piping of this section, the piping as it existed at the completion of the study, and our recommendations for any future work. The piping mostly consists of $1 / 4$ " OD, 0.035 " wall SS tubing and Swagelok fittings. The tubing size was actually selected to minimize plugging problems. At 0.57 GPM in the 0.18 " ID tubing, the velocity is $7.2 \mathrm{ft} / \mathrm{sec}$. This is a reasonable velocity from a pressure drop standpoint, and it fast enough to prevent the larger solids settling out in horizontal runs under flow conditions. In the past it has been sufficient to lift the solids off the bottom of the horizontal tubing runs after flow is stopped and subsequently restarted. Previous testing did not have major pluggage issues.

However, the new simulant appears to be much more prone to plugging piping. We have noted that the simulant seems to stick together if it is allowed to sit for short periods of time. When it is initially drained or pumped into a 5 gallon bucket it is quite easy to swirl up and pour out immediately, but after even 10 or 15 minutes it is more difficult to get the solids moving. As the P\&ID shows, we originally made some provisions to be able to flush out lines with water in case of a plug, but we had not expected to need to use the capability often. Instead, we found that if we simply turned off the transfer pump between transfers we were unlikely to be able to restart the flow even 30 minutes later to do another transfer. 


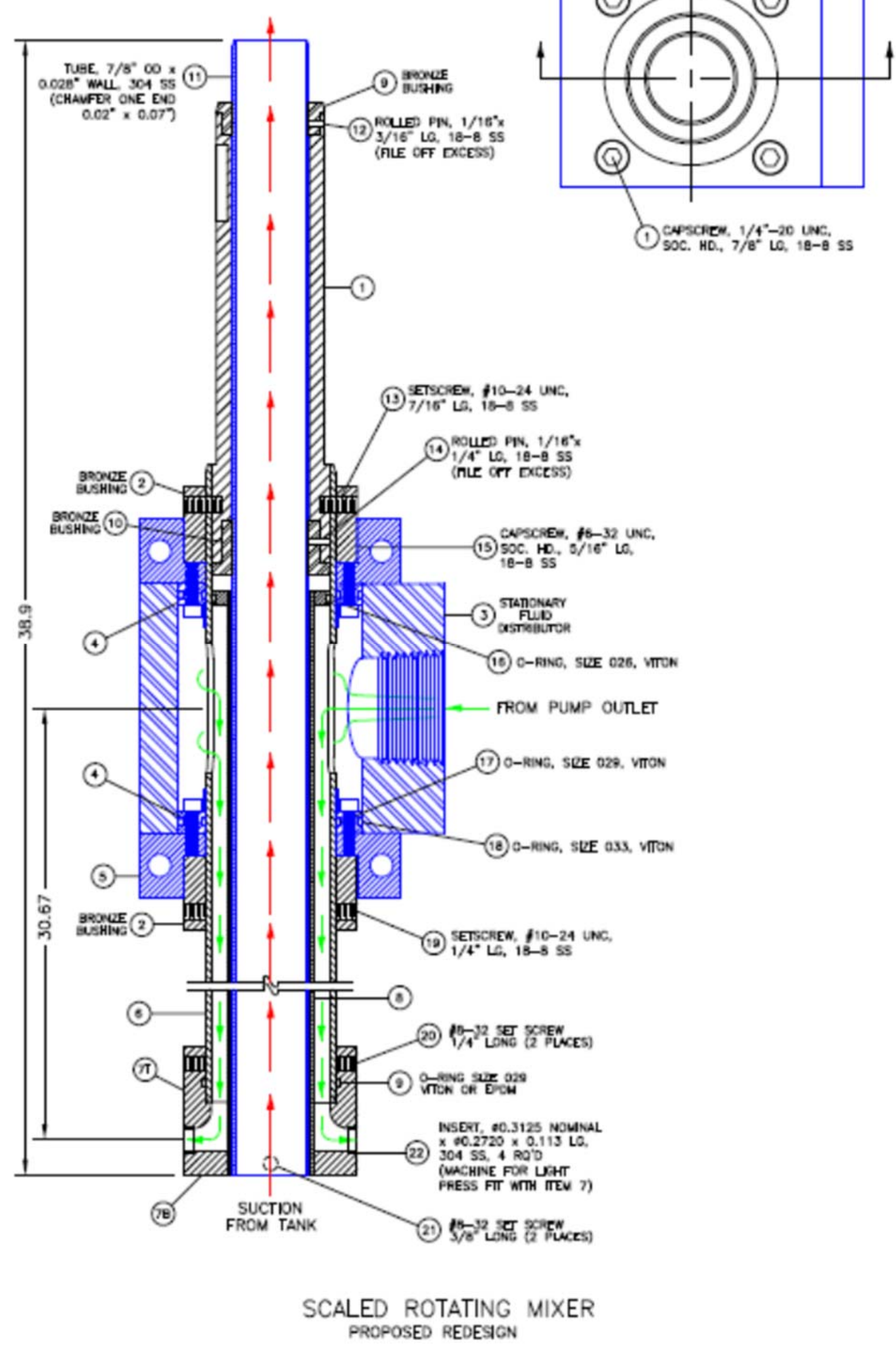

Figure C-0-3. Jet pump - new, but not implemented, design. 


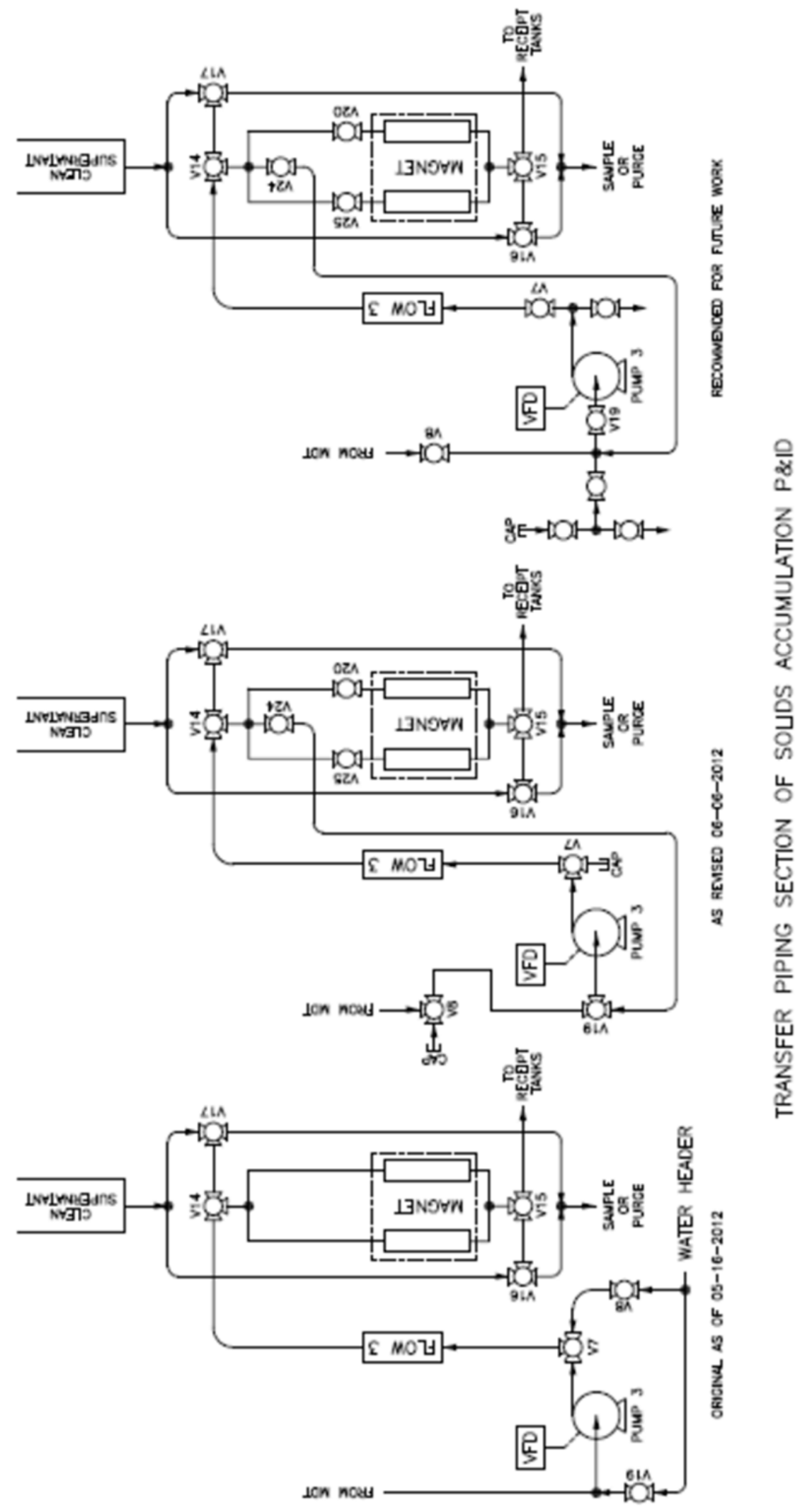

Figure C-0-4. Batch transfer system. 
We first removed disconnected the water header from valve V19 and removed valve V8. We changed valve V19 to a 3-way valve with the common leg connected to the transfer line from the MDT. That allowed us to blow down the transfer line from the MDT with air if we needed to without pressurizing the pump, and allowed us to flush the line with the flowmeter with clean supernatant through the path through V16, through V15, through the magnetic separator, through V14, through the flowmeter, and out V7 to a bucket.

We had one instance when we were either a little slow in removing the magnet blade from the magnetic separator and installing the dummy blade, or did not have the flow channels fully filled with supernatant during the transfer. A large amount of SS dropped to the bottom of one of the flow channels in the magnetic separator and blocked that exit. Although we had provided the ability to upflow through the channels to help dislodge a plug, the one open channel was able to bypass sufficient flow around the plugged channel so that the plug could not be removed. We installed valves V20 and V25 so we could shut off one flow path, and were then able to break up the plug and empty the magnetic separator. Subsequently, we ensured the flow channels were full of liquid when the magnet blade was removed and replaced with the dummy, and were careful to make the change quickly and start the purge flow immediately. With these operational changes, we had no further problems in flushing out the SS from the magnetic separator.

We continued to have difficulty with the pump plugging. We added valve V8, and reoriented valves V7 and V19 so the common legs were connected to the pump. That allowed us to maintain the ability to blow down the transfer line and added the ability to flush the pump through valve V7 to a bucket. We added valve V24 to provide the ability to flush water through V19, through the pump, through V7, through the flowmeter, through V14, and out V24 to a bucket. Even though we maintained the ability to blow down the transfer line from the MDT, we never had a problem requiring us to do so.

Although with these new valves we could flush the lines when needed, we found we were flushing with subsequent losses of supernatant more often that we wished. The final change of adding a line from valve 24 and V19 allowed us to maintain circulation through lines between transfers and eliminated almost all the plugging problems. Our procedure now, in general terms, is to:

1. Shut off the pump after each transfer

2. Purge out the non-SS solids from the magnetic separator

3. Partially purge the pump with a small amount of clean supernatant

4. Switch out the magnet blade with the dummy blade and purge the SS from the magnetic separator by down-flowing clean supernatant

5. Refill the magnetic separator by up-flowing clean supernatant

6. Valve in the recirculation line and restart the pump.

If these steps are performed quickly in sequence, there are seldom problems with line pluggage. At the end of the day the pump is run in recycle mode for a short while then shut off. The next day the pump generally starts transferring without problems.

When making the valve changes discussed above there were consideration with trying to minimize the changes needed in the R\&D directions as well as some restrictions due to already having chemicals in the valves and lines. The recommended P\&ID for future work assumes starting out with a clean system before all the operating procedures are written. In that case, use of one-way valves would offer more versatility than the chains of 3-way valves. Also, the use of three valves in a double block and bleed configuration connected to the cross before valve V19 
allows easier connection to either air or water headers as desired without some of the safety concerns while making the connections.

Hindsight tells us we should have paid more attention to changes in line sizes. For instance, the flowmeter we used for the transfers was a $6 \mathrm{~mm}$ (nearly 1/4") ID magnetic flowmeter. However, it was held between two 1/2" $150 \mathrm{lb}$ pipe flanges connected to the SS tubing with Swagelok tube to male NPT pipe connectors. There is a short distance between the end of the connector and the actual flowmeter channel that is the pipe tap drill diameter, 0.719 ". While the velocity in the tubing is $7.19 \mathrm{ft} / \mathrm{sec}$, the local velocity in this short gap falls to $0.451 \mathrm{ft} / \mathrm{sec}$. That slower velocity likely contributed to pluggage issues by allowing fast settling solids to accumulate around a small flow channel. When flow was stopped, those solids would drop down and jam up at the smaller tubing and flowmeter channel opening. It would have been prudent to drill through the Swagelok connector so the tubing could be pressed hard against the interior of the flowmeter before swaging the tubing in place. That simple change would greatly reduce the chance of a plug at that location.

Somewhat surprisingly, we did not experience any pluggage in the horizontal line leading to the Receipt Tanks. We also did not have any problems with pluggage in the larger piping that had much higher flow rates. We did have a few instances where the sight glass at the top of the Receipt Tanks plugged at the Swagelok elbow fitting connecting them to the PVC pipe section. While filling the Receipt Tanks, the level in the tank rose up several inches above the cut-off mark before the plug was washed out and the sight glass suddenly filled with liquid indicating the overfilled condition. We eliminated that problem as soon as it was discovered by washing out the sightglasses with a little clean supernatant soon after the Receipt Tanks were emptied out at the end of each day of operation.

\section{Tank Draining and Mound Measurements}

Originally, as one of the mound measuring techniques, we had planned to drain the heel liquid down slowly and in small increments through the bottom drain. At each increment we would photograph the mounds. By determining the "shoreline" in each picture and superimposing them, a contour map of the mounds could be generated and the volume could be approximated. What we didn't anticipate was that the fine Gibbsite and zirconia solids would coat everything so thickly that the shorelines would be very difficult to distinguish.

To avoid that problem, we changed our procedure to the following:

1. With the heel at 3.23 ", mix for approximately 15 minutes with the jet mixers at 8 gpm [Galigher set at $29 \mathrm{~Hz}$ ]with normal rotation rate of $1.6 \mathrm{rpm}$

2. Stop the mixer rotation when the jets are aimed along the line through the two mixers and reduce the jet mixer flow to approximately $3 \mathrm{gpm}$ [Galigher set at $12 \mathrm{~Hz}$ ]

3. Start pumping the liquid out of the tank through the normal transfer line using a peristaltic pump

4. When the liquid level reaches 2 " reduce the jet mixer flow to approximately 2 gpm [Galigher set at $10 \mathrm{~Hz}$ ]

5. When the liquid level reaches 1 " reduce the jet mixer flow to 1 gpm [Galigher set at 8 $\mathrm{Hz}]$

6. When the jets are exposed turn off the Galigher pump which drives the jet mixers

7. When the liquid level reaches the bottom of the transfer tube (about $1 / 4$ ") shut off the peristaltic pump and open the bottom drain valve 
8. Drain the remaining liquid until the surface tension stops the flow out the bottom drain (about 0.1" liquid level)

This draining method keeps the small solids suspended sufficiently so most of them can be removed from the tank leaving a much thinner coating over the mounds and tank bottom. With the tank drained, the laser measuring system is installed and used to collect data on the mound height and shape. After the laser measurements are complete, the core samples are collected. By this time the fine solids in the heel liquid removed will have settled sufficiently that mostly clear supernatant can be pumped back into the tank slowly and in small increments (typically 0.2"). Photographs are taken at each height increment to generate a contour map. The contrast between the fairly clean supernatant and mound material is sufficient that the "shoreline" is much more easily determined. When the mounds are covered, the remaining heel liquid is mixed up and pumped back into the MDT after the $1^{\text {st }}$ and $5^{\text {th }}$ cycles measurements, or all the material including the mounds is removed and the tank cleaned after the $10^{\text {th }}$ cycle measurements. 
Revision 0

\section{Appendix D: List of Videos Taken}


For each of the seven transfers of each cycle photographs were taken of the MDT bottom to quantify the footprints of the two mounds of accumulated solids. That is, for the two campaigns at least 280 photographs. Several are shown though this report and in general they all appear fairly similar, but are available on request. Furthermore, several videos of the bottom were taken to illustrate the action jet pumps and the interaction between the two jets. Starting with Cycle 2 of Campaign 2 videos were taken. The files are too large to transmit with the report, but can be sent separately if needed. The time length of the videos varies, but most show approximately 1 minute of jet pump mixing on the bottom of the MDT. For example for the first file, Camp2-Cycle2RT2.MOV, the time is 59 seconds in length for a file size of $72.7 \mathrm{MB}$. The file names should be self-explanatory but for example Camp2-Cycle2-RT2 = Campaign 2, Cycle 2, Receipt Tank 2 (That is, the period before and during the second batch transfer to Receipt Tank 2.)

\begin{tabular}{ll} 
File Name & $\underline{\text { File Size }}$ \\
\hline Camp2-Cycle2-RT2.MOV & $73 \mathrm{MB}$ \\
Camp2-Cycle2-RT3.MOV & $100 \mathrm{MB}$ \\
Camp2-Cycle2-RT4.MOV & $101 \mathrm{MB}$ \\
Camp2-Cycle2-RT5.MOV & $122 \mathrm{MB}$ \\
Camp2-Cycle2-RT6.MOV & $82 \mathrm{MB}$ \\
Camp2-Cycle2-Tank.MOV & $8 \mathrm{MB}$ \\
& \\
Camp2-Cycle3-RT1.MOV & $389 \mathrm{MB}$ \\
Camp2-Cycle3-RT2.MOV & $80 \mathrm{MB}$ \\
Camp2-Cycle3-RT3.MOV & $97 \mathrm{MB}$ \\
Camp2-Cycle3-RT4.MOV & $69 \mathrm{MB}$ \\
Camp2-Cycle3-RT6.MOV & $73 \mathrm{MB}$ \\
Camp2-Cycle3-RT7.MOV & $215 \mathrm{MB}$ \\
& \\
Camp2-Cycle4-RT1.MOV & $50 \mathrm{MB}$ \\
Camp2-Cycle4-RT2.MOV & $57 \mathrm{MB}$ \\
Camp2-Cycle4-RT3.MOV & $63 \mathrm{MB}$ \\
Camp2-Cycle4-RT4.MOV & $82 \mathrm{MB}$ \\
Camp2-Cycle4-RT5.MOV & $68 \mathrm{MB}$ \\
Camp2-Cycle4-RT6.MOV & $51 \mathrm{MB}$ \\
Camp2-Cycle4-RT7.MOV & $60 \mathrm{MB}$ \\
Camp2-Cycle5-RT1.MOV & $82 \mathrm{MB}$ \\
Camp2-Cycle5-RT2.MOV & $82 \mathrm{MB}$ \\
Camp2-Cycle5-RT3.MOV & $70 \mathrm{MB}$ \\
Camp2-Cycle5-RT4.MOV & $98 \mathrm{MB}$ \\
Camp2-Cycle5-RT5.MOV & $113 \mathrm{MB}$ \\
Camp2-Cycle5-RT6.MOV & $78 \mathrm{MB}$ \\
Camp2-Cycle5-RT7.MOV & $76 \mathrm{MB}$ \\
Camp2-Cycle6-RT1.MOV & $88 \mathrm{MB}$ \\
Camp2-Cycle6-RT2.MOV & $86 \mathrm{MB}$ \\
Camp2-Cycle6-RT3.MOV & $97 \mathrm{MB}$ \\
Camp2-Cycle6-RT4.MOV & $89 \mathrm{MB}$ \\
Camp2-Cycle6-RT5.MOV & $122 \mathrm{MB}$ \\
Camp2-Cycle6-RT6.MOV & $77 \mathrm{MB}$ \\
Camp2-Cycle6-RT7.MOV & $156 \mathrm{MB}$
\end{tabular}




$\begin{array}{ll}\text { Camp2-Cycle7-RT1.MOV } & 92 \mathrm{MB} \\ \text { Camp2-Cycle7-RT2.MOV } & 89 \mathrm{MB} \\ \text { Camp2-Cycle7-RT3.MOV } & 85 \mathrm{MB} \\ \text { Camp2-Cycle7-RT4.MOV } & 84 \mathrm{MB} \\ \text { Camp2-Cycle7-RT5.MOV } & 107 \mathrm{MB} \\ \text { Camp2-Cycle7-RT6.MOV } & 137 \mathrm{MB} \\ \text { Camp2-Cycle7-RT7.MOV } & 134 \mathrm{MB} \\ & \\ \text { Camp2-Cycle8-RT1.MOV } & 91 \mathrm{MB} \\ \text { Camp2-Cycle8-RT2.MOV } & 119 \mathrm{MB} \\ \text { Camp2-Cycle8-RT3.MOV } & 110 \mathrm{MB} \\ \text { Camp2-Cycle8-RT4.MOV } & 109 \mathrm{MB} \\ \text { Camp2-Cycle8-RT5.MOV } & 89 \mathrm{MB} \\ \text { Camp2-Cycle8-RT6.MOV } & 109 \mathrm{MB} \\ \text { Camp2-Cycle8-RT7.MOV } & 1,069 \mathrm{MB} \\ & \\ \text { Camp2-Cycle9-RT1.MOV } & 91 \mathrm{MB} \\ \text { Camp2-Cycle9-RT2.MOV } & 1,668 \mathrm{MB} \\ \text { Camp2-Cycle9-RT3.MOV } & 97 \mathrm{MB} \\ \text { Camp2-Cycle9-RT4.MOV } & 93 \mathrm{MB} \\ \text { Camp2-Cycle9-RT5.MOV } & 122 \mathrm{MB} \\ \text { Camp2-Cycle9-RT6.MOV } & 106 \mathrm{MB} \\ \text { Camp2-Cycle9-RT7.MOV } & 107 \mathrm{MB} \\ & \\ \text { Camp2-Cycle10-RT1.MOV } & 87 \mathrm{MB} \\ \text { Camp2-Cycle10-RT2.MOV } & 89 \mathrm{MB} \\ \text { Camp2-Cycle10-RT3.MOV } & 90 \mathrm{MB} \\ \text { Camp2-Cycle10-RT4.MOV } & 110 \mathrm{MB} \\ \text { Camp2-Cycle10-RT5.MOV } & 125 \mathrm{MB} \\ \text { Camp2-Cycle10-RT6.MOV } & 87 \mathrm{MB} \\ \text { Camp2-Cycle10-RT7.MOV } & 93 \mathrm{MB} \\ \text { Camp2-Cycle10-postRT7.MOV } & 14 \mathrm{MB} \\ & \end{array}$




\section{Distribution:}

K. M. Fox, 999-W

D. A. Crowley, 773-43A

S. D. Fink, 773-A

B. J. Giddings, 786-5A

M. R. Duignan, 786-5A

T. J. Steeper, 786-5A

J. L. Steimke, 786-5A

C. C. Herman, 999-W

D. J. Adamson, 999-W

S. L. Marra, 773-A

F. M. Pennebaker, 773-42A

JW. R. Wilmarth, 773-A

J. P. Vaughan, 773-41A
Hanford Customer:

M. G. Thien, Washington River Protection

Solutions

T. Wooley, Washington River Protection

Solutions

K. P. Lee, AREVA Federal Services 